\title{
Enantioselective $\beta$-Protonation by a Cooperative Catalysis Strategy
}

\author{
Michael H. Wang, Daniel T. Cohen, C. Benjamin Schwamb, Rama K. Mishra, and \\ Karl A. Scheidt * \\ Department of Chemistry, Center for Molecular Innovation and Drug Discovery, \\ Chemistry of Life Processes Institute \\ Northwestern University \\ 2170 Campus Drive, Evanston, IL 60208 (USA)
}

\section{Supporting Information}

\section{Table of Contents}

Table of Contents $\mathrm{S} 1$

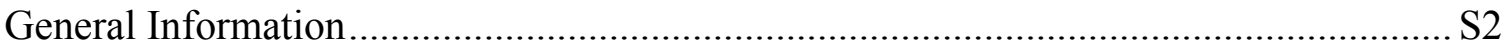

General Procedure for the Synthesis of Vinyl Iodide ................................................ S2

General Procedure for the Synthesis of Unsaturated Aldehyde.................................... S3

Optimization Table for Enantioselective $\beta$-Protonation ......................................... S11

General Procedure A for the Synthesis of Succinic Diester ...................................... S13

General Procedure B for the Synthesis of Succinic Diester ..................................... S13

General Procedures for the Synthetic Transformations ….................................... S22

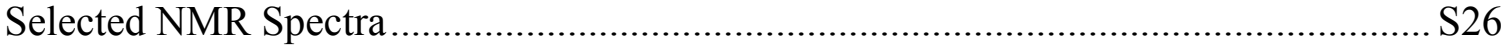

HPLC Traces of Racemic and Enantioenriched Compounds .................................... S71

Control experiment with $Z$-isomer starting material........................................... S104

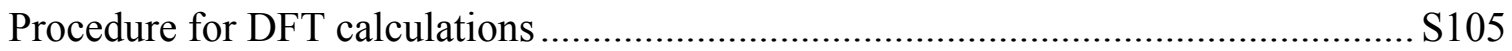

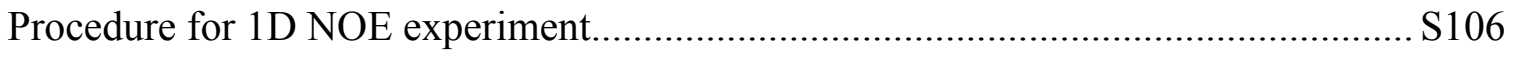

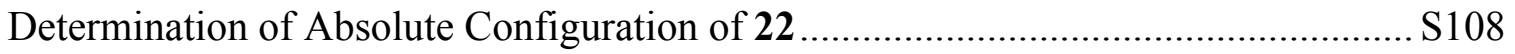




\section{General Information}

All reactions were carried out under a nitrogen atmosphere in oven-dried glassware with magnetic stirring. THF, toluene, and DMF were purified by passage through a bed of activated alumina. ${ }^{1}$ Reagents were purified prior to use unless otherwise stated following the guidelines of Perrin and Armarego. ${ }^{2}$ 1,2-Dichloroethane (DCE) was distilled from $\mathrm{CaH}_{2}$. Purification of reaction products was carried out by flash chromatography using EM Reagent silica gel 60 (230-400 mesh). Analytical thin layer chromatography was performed on EM Reagent $0.25 \mathrm{~mm}$ silica gel $60-\mathrm{F}$ plates. Visualization was accomplished with UV light and ceric ammonium nitrate stain or potassium permangenate stain followed by heating. Infrared spectra were recorded on a Bruker Tensor 37 FT-IR spectrometer. ${ }^{1} \mathrm{H}$ NMR spectra were recorded on AVANCE III 500 $\mathrm{MHz}$ / direct cryoprobe $(500 \mathrm{MHz})$ spectrometer and are reported in ppm using solvent as an internal standard $\left(\mathrm{CDCl}_{3}\right.$ at $\left.7.26 \mathrm{ppm}\right)$. Data are reported as $(\mathrm{ap}=$ apparent, $\mathrm{s}=$ singlet, $\mathrm{d}=$ doublet, $\mathrm{t}=$ apparent triplet, $\mathrm{q}=$ quartet, $\mathrm{m}=$ multiplet, $\mathrm{b}=$ broad; coupling constant(s) in Hz; integration.) Proton-decoupled ${ }^{13} \mathrm{C}$ NMR spectra were recorded on an AVANCE III $500 \mathrm{MHz}$ w/ direct cryoprobe $(125 \mathrm{MHz})$ spectrometer and are reported in ppm using solvent as an internal standard $\left(\mathrm{CDCl}_{3}\right.$ at $\left.77.16 \mathrm{ppm}\right) .{ }^{19} \mathrm{~F}$ NMR spectra were acquired at $26^{\circ} \mathrm{C}$ on a $400 \mathrm{MHz}$ Agilent 400MR-DD2 spectrometer equipped with a OneNMR probe and a 7600AS autosampler; this system was funded by NSF CRIF grant CHE-104873. Optical rotations were measured on a Perkin Elmer Model 341 Polarimeter with a sodium lamp. Mass spectra were obtained on a WATERS Acquity-H UPLC-MS with a single quad detector (ESI) or on a Varian 1200 Quadrupole Mass Spectrometer and Micromass Quadro II Spectrometer (ESI).

Triazolium precatalyst $\mathbf{A}$ is commercially available. Co-catalyst HBD3 was synthesized according to Taylor, et. al. ${ }^{3}$

\section{General Procedure for the Synthesis of Vinyl Iodide}

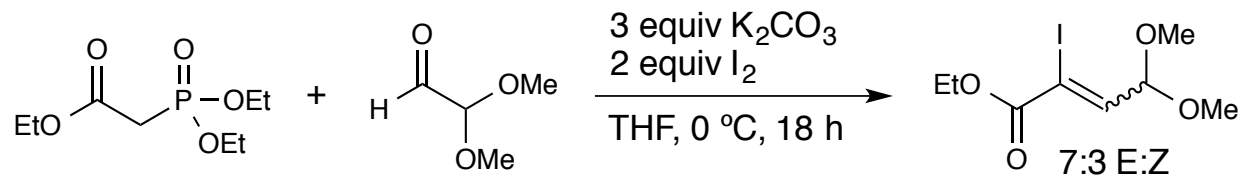

To a mixture of ethyl 2-(diethoxyphosphoryl)acetate (10 g, $44.6 \mathrm{mmol}, 1$ equiv), $\mathrm{K}_{2} \mathrm{CO}_{3}$ (18.49 g, $134 \mathrm{mmol}, 3$ equiv), and iodine (22.64 g, $89 \mathrm{mmol}, 2$ equiv) in THF (112 ml, $0.4 \mathrm{M}$ ) at $0{ }^{\circ} \mathrm{C}$ was added 2,2-dimethoxyacetaldehyde $\left(60\right.$ wt. \% in $\mathrm{H}_{2} \mathrm{O}, 8.51 \mathrm{~g}, 49.1$ mmol, 1.1 equiv) dropwise over $1 \mathrm{~h}$. The mixture was allowed to warm to $23{ }^{\circ} \mathrm{C}$ overnight. Conversion was monitored by ${ }^{1} \mathrm{H} \mathrm{NMR}\left(\mathrm{CDCl}_{3}, 500 \mathrm{MHz}\right)$. After complete

[1] Pangborn, A. B.; Giardello, M. A.; Grubbs, R. H.; Rosen, R. K.; Timmers F. J., Organometallics 1996, 15, 1518-1520.

[2] Perrin, D. D.; Armarego, W. L. Purification of Laboratory Chemicals; 3rd Ed., Pergamon Press, Oxford. 1988.

[3] Rostami, A.; Colin, A.; Li, X. Y.; Chudzinski, M. G.; Lough, A. J.; Taylor, M. S. J. Org. Chem. 2010, 75, 3983-3992. 
conversion, the mixture was filtered through Celite and eluted with $\mathrm{Et}_{2} \mathrm{O}$. The organic phase was washed with saturated $\mathrm{Na}_{2} \mathrm{~S}_{2} \mathrm{O}_{3}$ solution, water, and brine, and then dried over $\mathrm{Na}_{2} \mathrm{SO}_{4}$ and concentrated. The crude mixture was purified by flash column chromatography on silica gel (10\% EtOAc/hex; TLC 20\% EtOAc/hex, UV, $\mathrm{KMnO}_{4}$ or $\mathrm{CAM}, \mathrm{R}_{\mathrm{f}}$ 0.7) to afford ethyl 2-iodo-4,4-dimethoxybut-2-enoate (12.6 g, $41.9 \mathrm{mmol}$, $94 \%$ ) as a clear yellow oil as a 7:3 mixture of $\mathrm{Z}: \mathrm{E}$ isomers.<smiles>CCOC(=O)/C=C(\I)OC</smiles>

Ethyl 2-iodo-4,4-dimethoxybut-2-enoate: Prepared according to the general procedure using ethyl 2-(diethoxyphosphoryl)acetate (10 g, $44.6 \mathrm{mmol})$ and purified by flash column chromatography on silica gel $(10 \%$ EtOAc/hex; TLC $20 \%$ EtOAc/hex, UV, $\mathrm{KMnO}_{4}$ or CAM, $\mathrm{R}_{\mathrm{f}}$ 0.7) to afford ethyl 2-iodo-4,4-dimethoxybut-2-enoate (12.6 g, 41.9 mmol, 94\%) as a clear yellow oil as a 7:3 mixture of $\mathrm{Z}$ : $\mathrm{E}$ isomers. Analytical data: $\mathrm{Z}$ isomer: ${ }^{1} \mathrm{H}$ NMR $\left(500 \mathrm{MHz}, \mathrm{CDCl}_{3}\right) \delta 7.23(\mathrm{~d}, \mathrm{~J}=6.2 \mathrm{~Hz}, 1 \mathrm{H}), 5.03(\mathrm{~d}, \mathrm{~J}=6.2 \mathrm{~Hz}, 1 \mathrm{H})$, $3.42(\mathrm{~s}, 6 \mathrm{H}), 1.33(\mathrm{t}, \mathrm{J}=7.1 \mathrm{~Hz}, 3 \mathrm{H})$. E isomer: ${ }^{1} \mathrm{H}$ NMR $\left(500 \mathrm{MHz}, \mathrm{CDCl}_{3}\right) \delta 6.70(\mathrm{~d}, \mathrm{~J}$ $=6.0 \mathrm{~Hz}, 1 \mathrm{H}), 5.34(\mathrm{~d}, \mathrm{~J}=6.0 \mathrm{~Hz}, 1 \mathrm{H}), 3.36(\mathrm{~s}, 6 \mathrm{H}), 1.34(\mathrm{t}, \mathrm{J}=5.3 \mathrm{~Hz}, 3 \mathrm{H}) .{ }^{13} \mathrm{C} \mathrm{NMR}$ $\left(126 \mathrm{MHz}, \mathrm{CDCl}_{3}\right) \delta 164.0,162.6,148.2,146.5,105.8,100.6,97.3,89.3,63.3,62.7$, 53.8, 53.7, 14.3, 14.1; IR (film) 2982, 2935, 2830, 1717, 1622, 1444, 1366, 1347, 1298, 1237, 1191, 1122, 1089, 1053,1033, 973, 912, 863, 748, 671, 639, 614, $606 \mathrm{~cm}^{-1}$; LRMS (ESI): Mass calcd for $\mathrm{C}_{7} \mathrm{H}_{10} \mathrm{IO}_{3}[\mathrm{M}-\mathrm{OMe}]^{+}$: 269; found 269 .

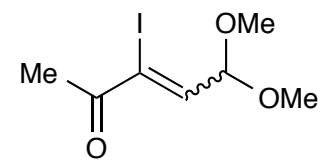

(Z)-3-iodo-5,5-dimethoxypent-3-en-2-one: Prepared according to the general procedure using diethyl (2-oxopropyl)phosphonate ( $2 \mathrm{~g}, 10.30 \mathrm{mmol}, 1$ equiv) and purified by flash column chromatography on silica gel (10\% EtOAc/hex; TLC $20 \%$ EtOAc/hex, UV, $\left.\mathrm{KMnO}_{4}, \mathrm{R}_{\mathrm{f}} 0.5\right)$ to afford 3-iodo-5,5-dimethoxypent-3-en-2-one (1.2 g, $\left.4.32 \mathrm{mmol}, 42 \%\right)$ as a clear yellow oil as a $>20: 1$ mixture of $\mathrm{Z}$ : $\mathrm{E}$ isomers. Analytical data: ${ }^{1} \mathrm{H}$ NMR (500 $\left.\mathrm{MHz}, \mathrm{CDCl}_{3}\right) \delta 6.97(\mathrm{~d}, \mathrm{~J}=6.0 \mathrm{~Hz}, 1 \mathrm{H}), 5.12(\mathrm{~d}, \mathrm{~J}=6.0 \mathrm{~Hz}, 1 \mathrm{H}), 3.44(\mathrm{~s}, 6 \mathrm{H}), 2.52(\mathrm{~s}$, $3 \mathrm{H}) ;{ }^{13} \mathrm{C} \mathrm{NMR}\left(126 \mathrm{MHz}, \mathrm{CDCl}_{3}\right) \delta 192.6,146.3,113.0,106.6,54.2,25.2$; IR (film) 2996, 2934, 2831, 1687, 1613, 1442, 1354, 1222, 1190, 1126, 1057, $970 \mathrm{~cm}^{-1}$; LRMS (ESI): Mass calcd for $\mathrm{C}_{6} \mathrm{H}_{8} \mathrm{IO}_{2}[\mathrm{M}-\mathrm{OMe}]^{+}$: 239; found 239.

\section{General Procedure for the Synthesis of Unsaturated Aldehyde}

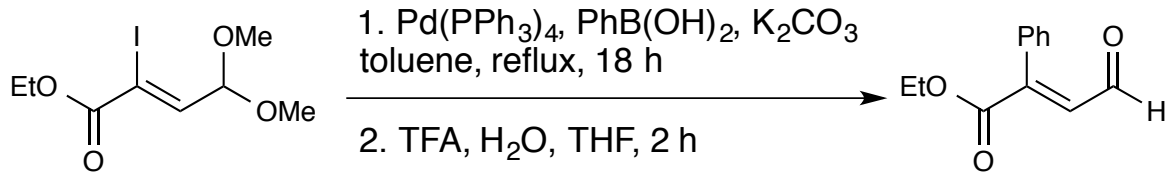

To a stirred suspension of (Z)-ethyl 2-iodo-4,4-dimethoxybut-2-enoate (3 g, $10.00 \mathrm{mmol}$, 1 equiv), phenylboronic acid (1.828 g, $15.00 \mathrm{mmol}, 1.5$ equiv), and $\mathrm{K}_{2} \mathrm{CO}_{3}$ (2.76 g, 19.99 mmol, 2 equiv) in toluene $(50.0 \mathrm{ml}, 0.2 \mathrm{M})$ under $\mathrm{N}_{2}$ was added $\mathrm{Pd}\left(\mathrm{PPh}_{3}\right)_{4}(0.058 \mathrm{~g}, 0.050$ 
mmol, 0.005 equiv) and the mixture was heated at reflux for $18 \mathrm{~h}$ and monitored by ${ }^{1} \mathrm{H}$ NMR $\left(\mathrm{CDCl}_{3}, 500 \mathrm{MHz}\right)$. When complete, the mixture was filtered through Celite and eluted with EtOAc. The filtrate was then washed with saturated $\mathrm{NaHCO}_{3}$ solution and brine. Dried over $\mathrm{Na}_{2} \mathrm{SO}_{4}$ and concentrated. The oil was then passed through a plug of silica, eluting with $10 \%$ EtOAc/hex and concentrated to give ethyl 4,4-dimethoxy-2phenylbut-2-enoate as a pale yellow oil, which was carried on without further purification. In a round-bottom flask equipped with stir bar and septum, the acetal was dissolved in THF (19.98 ml, $0.5 \mathrm{M})$ and $\mathrm{H}_{2} \mathrm{O}(7.20 \mathrm{ml}, 400 \mathrm{mmol}, 40$ equiv) was added. The mixture was cooled to $0{ }^{\circ} \mathrm{C}$, and trifluoroacetic acid $(7.70 \mathrm{ml}, 100 \mathrm{mmol}, 10$ equiv) was added dropwise via syringe. The solution was allowed to warm to $23{ }^{\circ} \mathrm{C}$, stirred for 2 $\mathrm{h}$, and monitored by ${ }^{1} \mathrm{H} \mathrm{NMR}\left(\mathrm{CDCl}_{3}, 500 \mathrm{MHz}\right)$. Once complete, the solution was diluted with $\mathrm{Et}_{2} \mathrm{O}$ and carefully neutralized with saturated $\mathrm{NaHCO}_{3}$ solution. The organic phase was separated, and the aqueous was extracted with $\mathrm{Et}_{2} \mathrm{O}(2 \times 25 \mathrm{~mL})$. The organics were combined and washed with brine. The crude was dried over $\mathrm{Na}_{2} \mathrm{SO}_{4}$, concentrated, and purified by flash column chromatography (10\% EtOAc/hex; TLC 20\% EtOAc/hex, $\mathrm{UV}, \mathrm{CAM}$ or $\left.\mathrm{KMnO} 4, \mathrm{R}_{\mathrm{f}} 0.6\right)$ to afford (E)-ethyl 4-oxo-2-phenylbut-2-enoate $(1.0 \mathrm{~g}$, $4.96 \mathrm{mmol}, 50 \%$ over 2 steps) as a yellow solid. Note: Several of the enal substrates will solidify upon standing over a period of 2 weeks. This process can be rapidly accelerated by azeotroping the oil with $\mathrm{Et}_{2} \mathrm{O}$, and then placing the oil in a $-30{ }^{\circ} \mathrm{C}$ freezer for $3-18 \mathrm{~h}$.

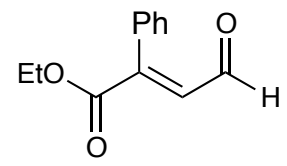

Ethyl $(E)$-4-oxo-2-phenylbut-2-enoate (1): Prepared according to the general procedure using phenylbronic acid ( $1.8 \mathrm{~g}, 15.0 \mathrm{mmol}, 1.5$ equiv) to afford $1.0 \mathrm{~g} \mathrm{(50 \%} \mathrm{yield} \mathrm{over} 2$ steps) of product as a yellow solid. Analytical data: ${ }^{1} \mathrm{H} \mathrm{NMR}\left(500 \mathrm{MHz}, \mathrm{CDCl}_{3}\right): \delta 9.66$ $(\mathrm{d}, J=7.8 \mathrm{~Hz}, 1 \mathrm{H}), 7.56-7.42(\mathrm{~m}, 3 \mathrm{H}), 7.42-7.35(\mathrm{~m}, 2 \mathrm{H}), 7.01(\mathrm{~d}, J=7.8 \mathrm{~Hz}, 1 \mathrm{H})$, $4.35(\mathrm{q}, J=7.1 \mathrm{~Hz}, 2 \mathrm{H}), 1.36(\mathrm{t}, J=7.1 \mathrm{~Hz}, 3 \mathrm{H}) ;{ }^{13} \mathrm{C} \mathrm{NMR}\left(125 \mathrm{MHz} ; \mathrm{CDCl}_{3}\right)$ : $\delta 193.68$, 166.59, 149.66, 134.75, 131.91, 130.60(2C), 129.91, 128.37(2C), 62.40, 14.23; IR (film) 3059, 2983, 2939, 2850, 2757, 1721, 1682, 1608, 1574, 1495, 1466, 1444, 1390, 1369, 1340, 1299, 1175, 1106, 1025, 1000, 865, 782, 712, $696 \mathrm{~cm}^{-1}$; LRMS (ESI): Mass calcd for $\mathrm{C}_{12} \mathrm{H}_{13} \mathrm{O}_{3}[\mathrm{M}+\mathrm{H}]^{+}$: 205; found 205 .<smiles>CCOC(=O)/C(=C/C=O)c1ccc2ccccc2c1</smiles>

Ethyl (E)-2-(naphthalen-2-yl)-4-oxobut-2-enoate: Prepared according to the general procedure using naphthalen-2-ylboronic acid (1.72 $\mathrm{g}, 10 \mathrm{mmol}, 1.5 \mathrm{equiv})$ to afford to

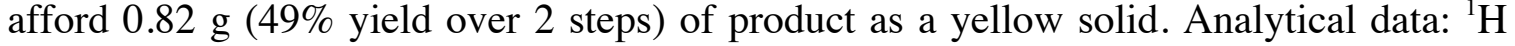
NMR $\left(500 \mathrm{MHz} ; \mathrm{CDCl}_{3}\right): \delta 9.67(\mathrm{~d}, J=7.7 \mathrm{~Hz}, 1 \mathrm{H}), 7.94-7.83(\mathrm{~m}, 3 \mathrm{H}), 7.85-7.77$ $(\mathrm{m}, 1 \mathrm{H}), 7.62-7.53(\mathrm{~m}, 2 \mathrm{H}), 7.47(\mathrm{dd}, J=8.5,1.8 \mathrm{~Hz}, 1 \mathrm{H}), 7.07(\mathrm{~d}, J=7.8 \mathrm{~Hz}, 1 \mathrm{H})$, $4.35(\mathrm{q}, J=7.1 \mathrm{~Hz}, 2 \mathrm{H}), 1.34(\mathrm{t}, J=7.1 \mathrm{~Hz}, 3 \mathrm{H}) ;{ }^{13} \mathrm{C}$ NMR $\left(125 \mathrm{MHz} ; \mathrm{CDCl}_{3}\right): \delta 193.7$, 
$166.7,149.7,135.1,133.7,132.6,131.1,129.5,128.5,128.1,127.9,127.6,127.14$, 127.07, 62.5, 14.3; IR (film) 3060, 2983, 2932, 2906, 2852, 2749, 1721, 1679, 1600, 1505, 1469, 1446, 1391, 1370, 1325, 1241, 1209, 1185, 1164, 1130, 1102, 1035, 909, 863, 822, 779,732 $\mathrm{cm}^{-1}$; LRMS (ESI): Mass calcd for $\mathrm{C}_{16} \mathrm{H}_{15} \mathrm{O}_{3}[\mathrm{M}+\mathrm{H}]^{+}: 255$; found 255 .<smiles>CCOC(=O)/C(=C/C=O)c1ccc([N+](=O)[O-])cc1</smiles>

Ethyl (E)-4-oxo-2-( $\boldsymbol{p}$-tolyl)but-2-enoate: Prepared according to the general procedure using $p$-tolylboronic acid ( $0.997 \mathrm{~g}, 7.33 \mathrm{mmol}, 1.1$ equiv) to afford $0.9 \mathrm{~g}$ (62\% yield over 2 steps) of product as a yellow solid. Analytical data: ${ }^{1} \mathrm{H}$ NMR $\left(500 \mathrm{MHz}, \mathrm{CDCl}_{3}\right) \delta 9.64$ $(\mathrm{d}, \mathrm{J}=7.8 \mathrm{~Hz}, 1 \mathrm{H}), 7.25(\mathrm{~m}, 4 \mathrm{H}), 6.94(\mathrm{~d}, \mathrm{~J}=7.8 \mathrm{~Hz}, 1 \mathrm{H}), 4.32(\mathrm{q}, \mathrm{J}=7.1 \mathrm{~Hz}, 2 \mathrm{H}), 2.41$ $(\mathrm{s}, 3 \mathrm{H}), 1.33(\mathrm{t}, \mathrm{J}=7.1 \mathrm{~Hz}, 3 \mathrm{H}) ;{ }^{13} \mathrm{C} \mathrm{NMR}\left(126 \mathrm{MHz}, \mathrm{CDCl}_{3}\right) \delta 193.8,166.8,149.7$, 140.3, 134.4, 130.7(2C), 129.1(2C), 129.0, 62.3, 21.5, 14.2; IR (film) 3030, 2982, 2940, 2923, 2848, 2755, 1721, 1682, 1612, 1476, 1390, 1338, 1280, 1107, 1094, 1001, 829, 732 $\mathrm{cm}^{-1}$; LRMS (ESI): Mass calcd for $\mathrm{C}_{13} \mathrm{H}_{15} \mathrm{O}_{3}[\mathrm{M}+\mathrm{H}]^{+}$: 219; found 219.<smiles>CCOC(=O)/C(=C/C=O)c1cccc(OC)c1</smiles>

Ethyl $(\boldsymbol{E})$-4-oxo-2-( $\boldsymbol{m}$-tolyl)but-2-enoate: Prepared according to the general procedure using $m$-tolylboronic acid (1.994 g, $14.66 \mathrm{mmol}, 1.1 \mathrm{equiv}$ ) to afford $1.1 \mathrm{~g}$ (38\% yield over 2 steps) of product as a yellow solid. Analytical data: $1 \mathrm{H} \mathrm{NMR}\left(500 \mathrm{MHz}, \mathrm{CDCl}_{3}\right) \delta$ $9.64(\mathrm{~d}, \mathrm{~J}=7.7 \mathrm{~Hz}, 1 \mathrm{H}), 7.34(\mathrm{t}, \mathrm{J}=7.5 \mathrm{~Hz}, 1 \mathrm{H}), 7.31-7.26(\mathrm{~m}, 1 \mathrm{H}), 7.16(\mathrm{~d}, \mathrm{~J}=8.5 \mathrm{~Hz}$, $2 \mathrm{H}), 6.96(\mathrm{~d}, \mathrm{~J}=7.8 \mathrm{~Hz}, 1 \mathrm{H}), 4.33(\mathrm{q}, \mathrm{J}=7.1 \mathrm{~Hz}, 2 \mathrm{H}), 2.41(\mathrm{~s}, 3 \mathrm{H}), 1.34(\mathrm{t}, \mathrm{J}=7.1 \mathrm{~Hz}$, 3H); 13C NMR (126 MHz, $\left.\mathrm{CDCl}_{3}\right) \delta$ 193.8, 166.7, 149.9, 138.2, 134.6, 131.8, 131.2, 130.7, 128.3, 127.8, 62.4, 21.5, 14.2; IR (film) 3058, 2982, 2940, 2923, 2849, 2754, 1720, 1687, 1559, 1582, 1389, 1344, 1334, 1238, 1151, 1109, 1037, 999, 861, 797, 724, $683 \mathrm{~cm}^{-1}$; LRMS (ESI): Mass calcd for $\mathrm{C}_{13} \mathrm{H}_{15} \mathrm{O}_{3}[\mathrm{M}+\mathrm{H}]^{+}: 219$; found 219.<smiles>CCOC(=O)/C(=C/C=O)c1ccc(OC)cc1</smiles>

Ethyl (E)-2-(4-methoxyphenyl)-4-oxobut-2-enoate: Prepared according to the general procedure using (4-methoxyphenyl)boronic acid (0.608 $\mathrm{g}, 0.40 \mathrm{mmol}, 1.2 \mathrm{equiv})$ to afford $0.448 \mathrm{~g}\left(58 \%\right.$ yield over 2 steps) of product as a yellow oil. Analytical data: ${ }^{1} \mathrm{H}$ NMR (500 MHz; $\left.\mathrm{CDCl}_{3}\right): \delta 9.66(\mathrm{~d}, \mathrm{~J}=7.8 \mathrm{~Hz}, 1 \mathrm{H}), 7.31(\mathrm{~d}, \mathrm{~J}=8.8 \mathrm{~Hz}, 2 \mathrm{H}), 6.94(\mathrm{dd}, \mathrm{J}$ 
$=18.8,8.3 \mathrm{~Hz}, 3 \mathrm{H}), 4.32(\mathrm{q}, \mathrm{J}=7.1 \mathrm{~Hz}, 2 \mathrm{H}), 3.86(\mathrm{~s}, 3 \mathrm{H}), 1.34(\mathrm{t}, \mathrm{J}=7.1 \mathrm{~Hz}, 3 \mathrm{H}) ;{ }^{13} \mathrm{C}$ NMR (125 MHz; $\left.\mathrm{CDCl}_{3}\right): \delta$ 193.7, 193.5, 168.8, 153.6, 135.7, 134.7, 134.1, 129.0(2C), 128.8(2C), 62.1, 52.8, 31.6, 14.1; IR (film) 2982, 2962, 2937, 2900, 2841, 2755, $1719,1675,1605,1569,1512,1464,1443,1389,1369,1338,1294,1242,1174,1107$, 1027, 910, 837, $772 \mathrm{~cm}^{-1}$; LRMS (ESI): Mass calcd for $\mathrm{C}_{13} \mathrm{H}_{15} \mathrm{O}_{4}[\mathrm{M}+\mathrm{H}]^{+}:$235; found 235.<smiles>CCOC(=O)/C=C/C=O</smiles>

Ethyl (E)-2-(3,4-dimethoxyphenyl)-4-oxobut-2-enoate: Prepared according to the general procedure using (3,4-dimethoxyphenyl)boronic acid (1.09 g, $6 \mathrm{mmol}, 1.2$ equiv) to afford $483 \mathrm{mg}$ (36\% yield over 2 steps) of product as a yellow oil. Analytical data: ${ }^{1} \mathrm{H}$ NMR $\left(500 \mathrm{MHz}, \mathrm{CDCl}_{3}\right) \delta 9.66(\mathrm{~d}, \mathrm{~J}=7.6 \mathrm{~Hz}, 1 \mathrm{H}), 6.95-6.87(\mathrm{~m}, 4 \mathrm{H}), 4.33(\mathrm{q}, \mathrm{J}=7.1$ $\mathrm{Hz}, 2 \mathrm{H}), 3.93(\mathrm{~s}, 3 \mathrm{H}), 3.90(\mathrm{~s}, 3 \mathrm{H}), 1.34(\mathrm{t}, \mathrm{J}=7.1 \mathrm{~Hz}, 3 \mathrm{H}) ;{ }^{13} \mathrm{C}$ NMR $\left(126 \mathrm{MHz}, \mathrm{CDCl}_{3}\right)$ ठ 193.8, 166.9, 150.8, 149.2, 148.7, 134.2, 124.6, 124.4, 113.3, 110.6, 62.3, 56.1, 14.3; IR (film) 2985, 2963, 2937, 2906,2840, 1720, 1676, 1600, 1579, 1516, 1464, 1388, 1369, 1350, 1321, 1259, 1144, 1108, 1025, 914, 865, 816, 778, 731, $635 \mathrm{~cm}^{-1}$; LRMS (ESI): Mass calcd for $\mathrm{C}_{14} \mathrm{H}_{17} \mathrm{O}_{5}[\mathrm{M}+\mathrm{H}]^{+}: 265$; found 265 .<smiles>CCOC(=O)/C(=C/C=O)c1ccc(F)cc1</smiles>

Ethyl (E)-2-(4-fluorophenyl)-4-oxobut-2-enoate: Prepared according to the general procedure using (4-fluorophenyl)boronic acid $(0.513 \mathrm{~g}, 3.67 \mathrm{mmol}, 1.1$ equiv) to afford $0.435 \mathrm{~g}\left(59 \%\right.$ yield over 2 steps) of product as a yellow solid. Analytical data: ${ }^{1} \mathrm{H} \mathrm{NMR}$ $\left(500 \mathrm{MHz} ; \mathrm{CDCl}_{3}\right): \delta 9.66(\mathrm{~d}, J=7.8 \mathrm{~Hz}, 1 \mathrm{H}), 7.42-7.33(\mathrm{~m}, 2 \mathrm{H}), 7.22-7.11(\mathrm{~m}, 2 \mathrm{H})$, $7.02(\mathrm{~d}, J=7.7 \mathrm{~Hz}, 1 \mathrm{H}), 4.35(\mathrm{q}, J=7.1 \mathrm{~Hz}, 2 \mathrm{H}), 1.36(\mathrm{t}, J=7.1 \mathrm{~Hz}, 3 \mathrm{H}) ;{ }^{13} \mathrm{C} \mathrm{NMR}(125$ $\left.\mathrm{MHz} ; \mathrm{CDCl}_{3}\right): \delta 193.2,166.4,163.7\left(\mathrm{~d}, J_{\mathrm{C}-\mathrm{F}}=250.9 \mathrm{~Hz}\right), 148.3,135.0(2 \mathrm{C}), 132.6(2 \mathrm{C}, \mathrm{d}$, $\left.J_{\mathrm{C}-\mathrm{F}}=8.4 \mathrm{~Hz}\right), 127.9\left(\mathrm{~d}, J_{\mathrm{C}-\mathrm{F}}=3.5 \mathrm{~Hz}\right), 115.6\left(\mathrm{~d}, J_{\mathrm{C}-\mathrm{F}}=21.9 \mathrm{~Hz}\right), 62.5,14.2 ;{ }^{19} \mathrm{~F} \mathrm{NMR}$ $\left(376 \mathrm{MHz} ; \mathrm{CDCl}_{3}\right.$ ): $\delta-110.5$ (m); IR (film) 3108, 3075, 2985, 2938, 2917, 2850, 2758, 1723, 1684, 1601, 1508, 1472, 1447, 1394, 1369, 1338, 1301, 1242, 1176, 1163, 1106, 1031, 1015, 981, 866, 843, 772, $738 \mathrm{~cm}^{-1}$; LRMS (ESI): Mass calcd for $\mathrm{C}_{12} \mathrm{H}_{12} \mathrm{O}_{3} \mathrm{~F}$ $[\mathrm{M}+\mathrm{H}]^{+}:$223; found 223 . 


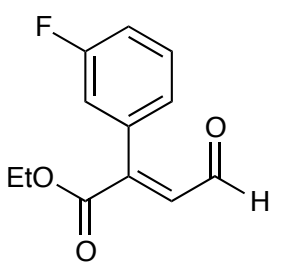

Ethyl (E)-2-(3-fluorophenyl)-4-oxobut-2-enoate: Prepared according to the general procedure using (3-fluorophenyl)boronic acid $(0.466 \mathrm{~g}, 3.33 \mathrm{mmol}, 1.0$ equiv) to afford $0.384 \mathrm{~g}(52 \%$ yield over 2 steps $)$ of product as a yellow oil. Analytical data: ${ }^{1} \mathrm{H}$ NMR $\left(500 \mathrm{MHz} ; \mathrm{CDCl}_{3}\right): \delta 9.64(\mathrm{~d}, J=7.8 \mathrm{~Hz}, 1 \mathrm{H}), 7.42(\mathrm{~m}, 1 \mathrm{H}), 7.22-7.07(\mathrm{~m}, 3 \mathrm{H}), 7.00$ $(\mathrm{d}, J=7.8 \mathrm{~Hz}, 1 \mathrm{H}), 4.32(\mathrm{q}, J=7.1 \mathrm{~Hz}, 2 \mathrm{H}), 1.36(\mathrm{t}, J=7.1 \mathrm{~Hz}, 3 \mathrm{H}) ;{ }^{13} \mathrm{C}$ NMR $(125$ $\left.\mathrm{MHz} ; \mathrm{CDCl}_{3}\right): \delta 193.1,166.0,162.4\left(\mathrm{~d}, J_{\mathrm{C}-\mathrm{F}}=248.0 \mathrm{~Hz}\right), 148.1\left(\mathrm{~d}, J_{\mathrm{C}-\mathrm{F}}=2.1 \mathrm{~Hz}\right), 135.3$, $133.8\left(\mathrm{~d}, J_{\mathrm{C}-\mathrm{F}}=8.1 \mathrm{~Hz}\right), 130.0\left(\mathrm{~d}, J_{\mathrm{C}-\mathrm{F}}=8.4 \mathrm{~Hz}\right), 126.5\left(\mathrm{~d}, J_{\mathrm{C}-\mathrm{F}}=3.1 \mathrm{~Hz}\right), 117.5\left(\mathrm{~d}, J_{\mathrm{C}-\mathrm{F}}=\right.$ $22.8 \mathrm{~Hz}), 117.0\left(\mathrm{~d}, J_{\mathrm{C}-\mathrm{F}}=21.1 \mathrm{~Hz}\right), 62.6,14.2 ;{ }^{19} \mathrm{~F} \mathrm{NMR}\left(376 \mathrm{MHz} ; \mathrm{CDCl}_{3}\right): \delta-112.3$ (m); IR (film) 3070, 2984, 2939, 2917, 2850, 2757, 2727, 1722, 1683, 1608, 1582, 1487, 1440, 1391, 1369, 1343, 1300, 1244, 1143, 1104, 1033, 1002, 906, 881, 795, 765, 726, $684 \mathrm{~cm}^{-1}$; LRMS (ESI): Mass calcd for $\mathrm{C}_{12} \mathrm{H}_{12} \mathrm{O}_{3} \mathrm{~F}[\mathrm{M}+\mathrm{H}]^{+}: 223$; found 223.

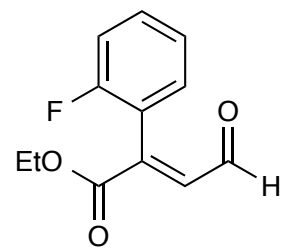

Ethyl (E)-2-(2-fluorophenyl)-4-oxobut-2-enoate: Prepared according to the general procedure using (2-fluorophenyl)boronic acid $(0.466 \mathrm{~g}, 3.33 \mathrm{mmol}, 1.0$ equiv) to afford $0.322 \mathrm{~g}$ (44\% yield over 2 steps) of product as a yellow oil. Analytical data: ${ }^{1} \mathrm{H}$ NMR $\left(500 \mathrm{MHz}, \mathrm{CDCl}_{3}\right) \delta 9.64(\mathrm{dd}, \mathrm{J}=7.9,1.5 \mathrm{~Hz}, 1 \mathrm{H}), 7.50-7.44(\mathrm{~m}, 1 \mathrm{H}), 7.30-7.27(\mathrm{~m}$, $1 \mathrm{H}), 7.23(\mathrm{td}, \mathrm{J}=7.5,1.0 \mathrm{~Hz}, 1 \mathrm{H}), 7.20-7.15(\mathrm{~m}, 1 \mathrm{H}), 7.06(\mathrm{~d}, \mathrm{~J}=7.9 \mathrm{~Hz}, 1 \mathrm{H}), 4.32(\mathrm{q}$, $\mathrm{J}=7.1 \mathrm{~Hz}, 2 \mathrm{H}), 1.31(\mathrm{t}, \mathrm{J}=7.1 \mathrm{~Hz}, 3 \mathrm{H}) ;{ }^{13} \mathrm{C} \mathrm{NMR}\left(126 \mathrm{MHz}, \mathrm{CDCl}_{3}\right) \delta 192.8,165.7$, $161.1,159.1,144.1,136.1,132.5,132.5,124.2,124.2,116.2,116.0,62.5,14.2$; IR (film) 2983, 2939, 2906, 2849, 1721, 1682, 1488, 1449, 1242, 1220, 1174, 1153, 1111, 1096, 1026, 837, 758, 748, $693 \mathrm{~cm}^{-1}$; LRMS (ESI): Mass calcd for $\mathrm{C}_{12} \mathrm{H}_{12} \mathrm{FO}_{3}[\mathrm{M}+\mathrm{H}]^{+}: 223$; found 223 .

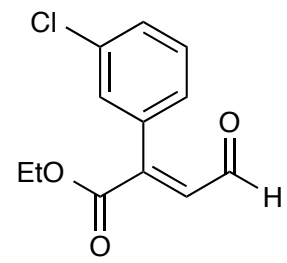

Ethyl (E)-2-(3-chlorophenyl)-4-oxobut-2-enoate: Prepared according to the general procedure using (3-chlorophenyl)boronic acid ( $0.860 \mathrm{~g}, 5.5 \mathrm{mmol}, 1.1$ equiv) to afford $0.620 \mathrm{~g}$ (52\% yield over 2 steps) of product as a yellow solid. Analytical data: ${ }^{1} \mathrm{H} \mathrm{NMR}$ $\left(500 \mathrm{MHz}, \mathrm{CDCl}_{3}\right) \delta 9.63(\mathrm{~d}, \mathrm{~J}=7.8 \mathrm{~Hz}, 1 \mathrm{H}), 7.45(\mathrm{~d}, \mathrm{~J}=8.7 \mathrm{~Hz}, 1 \mathrm{H}), 7.43-7.36(\mathrm{~m}$, 2H), $7.23(\mathrm{~d}, \mathrm{~J}=7.6 \mathrm{~Hz}, 1 \mathrm{H}), 7.01(\mathrm{~d}, \mathrm{~J}=7.8 \mathrm{~Hz}, 1 \mathrm{H}), 4.33(\mathrm{q}, \mathrm{J}=7.1 \mathrm{~Hz}, 2 \mathrm{H}), 1.33(\mathrm{t}, \mathrm{J}$ $=7.1 \mathrm{~Hz}, 3 \mathrm{H}) ;{ }^{13} \mathrm{C}$ NMR $\left(126 \mathrm{MHz}, \mathrm{CDCl}_{3}\right) \delta$ 193.0, 166.0, 148.0, 135.4, 134.6, 133.6, 130.4, 130.0, 129.7, 128.8, 62.6, 14.2; IR (film) 3067, 2983, 2938, 2906, 2850, 2754, 
1722, 1681, 1243, 1199, 1178, 1110, $1032 \mathrm{~cm}^{-1}$; LRMS (ESI): Mass calcd for $\mathrm{C}_{12} \mathrm{H}_{12} \mathrm{ClO}_{3}$ $[\mathrm{M}+\mathrm{H}]^{+}:$239; found 239 .<smiles>CCOC(=O)/C(=C/C=O)c1cccc([N+](=O)[O-])c1</smiles>

Ethyl (E)-2-(3-nitrophenyl)-4-oxobut-2-enoate: Prepared according to the general procedure using (3-nitrophenyl)boronic acid ( $1.40 \mathrm{~g}, 8.4 \mathrm{mmol}, 1.2 \mathrm{equiv})$ to afford 1.03 g (60\% yield over 2 steps) of product as a yellow solid. Analytical data: ${ }^{1} \mathrm{H}$ NMR (500 $\left.\mathrm{MHz} ; \mathrm{CDCl}_{3}\right): \delta 9.63(\mathrm{~d}, \mathrm{~J}=7.8 \mathrm{~Hz}, 1 \mathrm{H}), 8.34(\mathrm{dt}, \mathrm{J}=7.2,2.2 \mathrm{~Hz}, 1 \mathrm{H}), 8.26(\mathrm{~m}, 1 \mathrm{H})$, 7.72-7.60 (m, 2H), $7.11(\mathrm{~d}, \mathrm{~J}=7.8 \mathrm{~Hz}, 1 \mathrm{H}), 4.34(\mathrm{q}, \mathrm{J}=7.1 \mathrm{~Hz}, 2 \mathrm{H}), 1.34(\mathrm{t}, \mathrm{J}=7.1 \mathrm{~Hz}$, $3 \mathrm{H}) ;{ }^{13} \mathrm{C}$ NMR $\left(125 \mathrm{MHz} ; \mathrm{CDCl}_{3}\right): \delta$ 192.1, 165.5, 148.1, 146.6, 136.4, 136.1, 133.5, 129.6, 125.3, 124.6, 62.9, 14.2; IR (film) 3115, 3086, 2984, 2941, 2907, 2873, 2759, 1772, 1723, 1681, 1524, 1465, 1431, 1303, 1235, 1186, 1127, 1033, 1001, 908, 864, 811, $801,772,723,689,616 \mathrm{~cm}^{-1}$; LRMS (ESI): Mass calcd for $\mathrm{C}_{12} \mathrm{H}_{11} \mathrm{NO}_{5}[\mathrm{M}+\mathrm{H}]^{+}: 249$; found 249 .<smiles>CCOC(=O)/C(=C/C=O)c1cccs1</smiles>

Ethyl (Z)-4-oxo-2-(thiophen-2-yl)but-2-enoate: Prepared according to the general procedure using thiophen-2-ylboronic acid (1.02 g, $8.0 \mathrm{mmol}, 1.2$ equiv) to afford 0.716 g (51\% yield over 2 steps) of product as a yellow solid. Analytical data: ${ }^{1} \mathrm{H}$ NMR (500 $\left.\mathrm{MHz}, \mathrm{CDCl}_{3}\right) \delta 9.87(\mathrm{~d}, \mathrm{~J}=7.4 \mathrm{~Hz}, 1 \mathrm{H}), 7.63(\mathrm{dd}, \mathrm{J}=5.2,1.2 \mathrm{~Hz}, 1 \mathrm{H}), 7.27(\mathrm{~d}, \mathrm{~J}=1.3$ $\mathrm{Hz}, 1 \mathrm{H}), 7.13(\mathrm{dd}, \mathrm{J}=5.2,3.6 \mathrm{~Hz}, 1 \mathrm{H}), 6.95(\mathrm{~d}, \mathrm{~J}=7.4 \mathrm{~Hz}, 1 \mathrm{H}), 4.37(\mathrm{q}, \mathrm{J}=7.1 \mathrm{~Hz}, 2 \mathrm{H})$, $1.38(\mathrm{t}, \mathrm{J}=7.1 \mathrm{~Hz}, 3 \mathrm{H}) ;{ }^{13} \mathrm{C}$ NMR $\left(126 \mathrm{MHz} \mathrm{CDCl}_{3}\right) \delta 193.0,166.2,141.6,133.8,133.6$, 132.7, 131.7, 127.2, 62.7, 14.2; IR (film) 3098, 3083, 3061, 2988, 2942, 2905, 2885, $1710,1665,1627,1581,1512,1473,1423,1363,1321,1250,1239,1143,1110,1099$, 1089, 1056, 1027, 1002, 978, 885, 867, 853, 812, 788, 769, 746, 729, 679, $613 \mathrm{~cm}^{-1}$; LRMS (ESI): Mass calcd for $\mathrm{C}_{10} \mathrm{H}_{11} \mathrm{SO}_{3}[\mathrm{M}+\mathrm{H}]^{+}: 211$; found 211 .

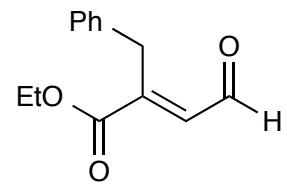

Ethyl (E)-2-benzyl-4-oxobut-2-enoate: To a $100 \mathrm{~mL}$ rbf was added ethyl 2(diethoxyphosphoryl)-3-phenylpropanoate $(1.2799 \mathrm{~g}, 4.07 \mathrm{mmol}, 1$ equiv), 2,2dimethoxyacetaldehyde $\left(1.060 \mathrm{~g}, 6.11 \mathrm{mmol}, 60 \mathrm{wt} \%\right.$ in $\mathrm{H}_{2} \mathrm{O}, 1.5$ equiv), heptane (10.18 $\mathrm{ml}, 0.4$ molar), and $\mathrm{K}_{2} \mathrm{CO}_{3}(0.844 \mathrm{~g}, 6.11 \mathrm{mmol}, 1.5$ equiv). The mixture was stirred and refluxed overnight. Monitored by ${ }^{1} \mathrm{H}$ NMR. Upon completion, reaction mixture was cooled to $23{ }^{\circ} \mathrm{C}$, diluted with $\mathrm{Et}_{2} \mathrm{O}$. The aqueous was separated and was back extracted. 
Combined organics was washed with water and then brine. Dried over $\mathrm{MgSO}_{4}$ and concentrated. The crude contained a $6: 4 \mathrm{E} / \mathrm{Z}$ ratio of products, and was carried on without further purification. The crude acetal was dissolved in acetone $(40 \mathrm{~mL}, 0.1$ molar) and water $(0.798 \mathrm{~mL})$ was added. Amberlyst 15 (0.50 g, approx. 0.5 equiv by weight $)$ was added and the mixture stirred overnight. Once complete, the Amberlyst was filtered off, and the filtrate concentrated. The crude was diluted with $\mathrm{Et}_{2} \mathrm{O}$, washed with saturated aqueous $\mathrm{NaHCO}_{3}$ solution, and brine, and then dried over $\mathrm{MgSO}_{4}$, filtered, and concentrated. Purification by flash column chromatography (10\% EtOAc/hexanes)

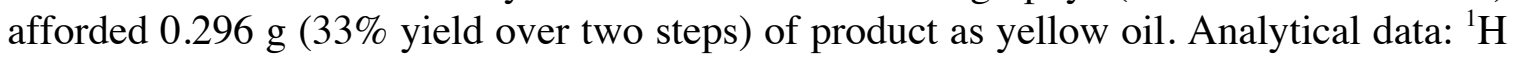
1H NMR (500 MHz, $\left.\mathrm{CDCl}_{3}\right) \delta 10.26(\mathrm{~d}, \mathrm{~J}=7.5 \mathrm{~Hz}, 1 \mathrm{H}), 7.29(\mathrm{~d}, \mathrm{~J}=7.1 \mathrm{~Hz}, 2 \mathrm{H}), 7.19$ $(\mathrm{d}, \mathrm{J}=7.0 \mathrm{~Hz}, 3 \mathrm{H}), 6.90(\mathrm{~d}, \mathrm{~J}=7.5 \mathrm{~Hz}, 1 \mathrm{H}), 4.20(\mathrm{q}, \mathrm{J}=7.1 \mathrm{~Hz}, 2 \mathrm{H}), 4.13(\mathrm{~s}, 2 \mathrm{H}), 1.24$ $(\mathrm{t}, \mathrm{J}=7.1 \mathrm{~Hz}, 3 \mathrm{H}) ;{ }^{13} \mathrm{C}$ NMR $\left(126 \mathrm{MHz}, \mathrm{CDCl}_{3}\right) \delta 191.9,166.8,148.0,137.3,134.1$, 128.9, 128.6, 127.1 , 62.1 , 32.7, 14.1; IR (film) 3063, 3029, 2982, 2938,2870, 2760, 1716, $1677,1600,1495,1453,1368,1315,1294,1248,1192,1124,1095,1077,1049,1029$, $1010,935,891,858,831,742,697 \mathrm{~cm}^{-1}$; LRMS (ESI): Mass calcd for $\mathrm{C}_{13} \mathrm{H}_{15} \mathrm{O}_{3}[\mathrm{M}+\mathrm{H}]^{+}$: 219; found 219.

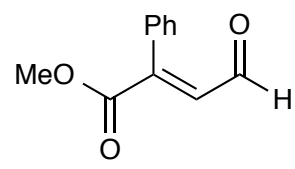

Methyl (E)-4-oxo-2-phenylbut-2-enoate: To a mixture of methyl 2(diethoxyphosphoryl)acetate (3.15 g, $15 \mathrm{mmol}), \mathrm{K}_{2} \mathrm{CO}_{3}(6.22 \mathrm{~g}, 45.0 \mathrm{mmol}, 3$ equiv), and iodine $\left(7.61 \mathrm{~g}, 30.0 \mathrm{mmol}, 2\right.$ equiv) in THF $(37.5 \mathrm{ml})$ at $0{ }^{\circ} \mathrm{C}$ was added 2,2dimethoxyacetaldehyde $\left(2.86 \mathrm{~g}, 16.50 \mathrm{mmol}, 60 \mathrm{wt} \%\right.$ in $\left.\mathrm{H}_{2} \mathrm{O}, 1.1 \mathrm{equiv}\right)$ dropwise. The mixture was allowed to warm to $23{ }^{\circ} \mathrm{C}$ overnight and then filtered through Celite and eluted with $\mathrm{Et}_{2} \mathrm{O}$. The filtrate was washed with $\mathrm{Na}_{2} \mathrm{~S}_{2} \mathrm{O}_{3}$, then water, and brine. Dried over $\mathrm{Na}_{2} \mathrm{SO}_{4}$, and concentrated. The crude was passed through a plug of silica, eluting with EtOAc, and carried on without purification. The crude was dissolved in toluene $(75 \mathrm{ml})$, and phenylboronic acid (2.74 g, $22.49 \mathrm{mmol}, 1.5$ equiv), $\mathrm{K}_{2} \mathrm{CO}_{3}$ (4.15 g, $30.0 \mathrm{mmol}, 2$ equiv), and $\mathrm{Pd}\left(\mathrm{Ph}_{3} \mathrm{P}\right)_{4}(0.087 \mathrm{~g}, 0.075 \mathrm{mmol}, 0.5 \mathrm{~mol} \%)$ were added. The flask was flushed with nitrogen, and then the mixture was heated at reflux for $18 \mathrm{~h}$. After confirming complete conversion by ${ }^{1} \mathrm{H}$ NMR, the mixture was filtered through Celite with a thin pad of silica on top, eluting with EtOAc. The filtrate was then washed with $\mathrm{NaHCO}_{3}$ and brine. Dried over $\mathrm{MgSO}_{4}$, concentrated, and carried on without purification. Finally, the crude was diluted with $\mathrm{H}_{2} \mathrm{O}(10.80 \mathrm{ml}, 599 \mathrm{mmol})$ and THF (Volume: 30.0 $\mathrm{ml}$ ), and trifluoroacetic acid (11.54 $\mathrm{ml}, 150 \mathrm{mmol}, 10$ equiv) was added dropwise. After stirring for $1 \mathrm{~h}$, the solution was diluted with $\mathrm{Et}_{2} \mathrm{O}$, carefully quenched with sat. aq. $\mathrm{NaHCO}_{3}$ and brine. The crude was dried over $\mathrm{Na}_{2} \mathrm{SO}_{4}$, filtered, concentrated, and purified by flash column chromatography (10\% EtOAc/hexanes) to afford $0.599 \mathrm{~g}$ (21\% yield over 3 steps) of product as yellow solid. Analytical data: ${ }^{1} \mathrm{H}$ NMR $\left(500 \mathrm{MHz}, \mathrm{CDCl}_{3}\right) \delta$ $9.63(\mathrm{~d}, \mathrm{~J}=7.8 \mathrm{~Hz}, 1 \mathrm{H}), 7.49-7.44(\mathrm{~m}, 3 \mathrm{H}), 7.36-7.33(\mathrm{~m}, 2 \mathrm{H}), 6.99(\mathrm{~d}, \mathrm{~J}=7.8 \mathrm{~Hz}$, $1 \mathrm{H}), 3.86(\mathrm{~s}, 3 \mathrm{H}) ;{ }^{13} \mathrm{C} \mathrm{NMR}\left(126 \mathrm{MHz}, \mathrm{CDCl}_{3}\right) \delta 193.5,167.1,149.3,135.1,131.9$, 130.5, 129.9, 128.4, 53.3; IR (film) 3060, 2958, 2887, 2849, 1719, 1667, 1606, 1595, 1573, 1495, 1445, 1435, 1396, 1352, 1242, 1190, 1167, 1104, 1079, 1036, 1018, 999, 971, 930, 908, 888, 862, 853, 784, 762, 716, 695, $611 \mathrm{~cm}^{-1}$; LRMS (ESI): Mass calcd for $\mathrm{C}_{11} \mathrm{H}_{11} \mathrm{O}_{3}[\mathrm{M}+\mathrm{H}]^{+}:$191; found 191 . 
<smiles>O=C/C=C(\C(=O)OCc1ccccc1)c1ccccc1</smiles>

Benzyl (E)-4-oxo-2-phenylbut-2-enoate: Prepared according to similar procedure as methyl (E)-4-oxo-2-phenylbut-2-enoate using benzyl 2-(diethoxyphosphoryl)acetate $(4.29 \mathrm{~g}, 15 \mathrm{mmol})$ to afford $1.89 \mathrm{~g}$ (47\% yield over 3 steps) of product as a yellow solid. Analytical data: ${ }^{1} \mathrm{H}$ NMR $\left(500 \mathrm{MHz} ; \mathrm{CDCl}_{3}\right): \delta 9.64(\mathrm{~d}, \mathrm{~J}=7.8 \mathrm{~Hz}, 1 \mathrm{H}), 7.51-7.33(\mathrm{~m}$, $10 \mathrm{H}), 7.01(\mathrm{~d}, \mathrm{~J}=7.7 \mathrm{~Hz}, 1 \mathrm{H}), 5.30(\mathrm{~s}, 2 \mathrm{H}) ;{ }^{13} \mathrm{C} \mathrm{NMR}\left(125 \mathrm{MHz} ; \mathrm{CDCl}_{3}\right): \delta 193.5$, 166.4, 149.3 , 135.2 , 135.0, 131.7, 130.6, 130.0, 128.8, 128.7, 128.4, 67.9; IR (film) 3066, 3034, 2959, 2848, 2758, 2721, 1718, 1678, 1498, 1260, 1170, 1105, 1010, 715, 696, 627, $618 \mathrm{~cm}^{-1}$; LRMS (ESI): Mass calcd for $\mathrm{C}_{17} \mathrm{H}_{15} \mathrm{O}_{3}[\mathrm{M}+\mathrm{H}]^{+}: 267$; found 267.

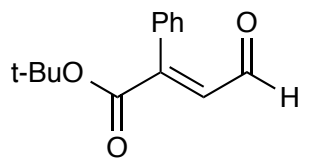

Tert-butyl (E)-4-oxo-2-phenylbut-2-enoate: Prepared according to similar procedure as methyl (E)-4-oxo-2-phenylbut-2-enoate using tert-butyl 2-(diethoxyphosphoryl)acetate $(2.018 \mathrm{~g}, 8 \mathrm{mmol})$ to afford $0.599 \mathrm{~g}(34 \%$ yield over 3 steps $)$ of product as a yellow oil. Analytical data: ${ }^{1} \mathrm{H}$ NMR $\left(500 \mathrm{MHz}, \mathrm{CDCl}_{3}\right) \delta 9.60(\mathrm{~d}, \mathrm{~J}=7.9 \mathrm{~Hz}, 1 \mathrm{H}), 7.43-7.41(\mathrm{~m}$, 2H), $7.32(\mathrm{dd}, \mathrm{J}=7.7,1.7 \mathrm{~Hz}, 3 \mathrm{H}), 6.87(\mathrm{~d}, \mathrm{~J}=7.9 \mathrm{~Hz}, 1 \mathrm{H}), 1.50(\mathrm{~s}, 9 \mathrm{H}) ;{ }^{13} \mathrm{C}$ NMR $(126$ $\left.\mathrm{MHz} \mathrm{CDCl}_{3}\right) \delta 194.0,165.6,151.2,134.1,132.3,130.6,129.7,128.3,83.2,28.1$; IR (film) 3059, 2978, 2933, 2846, 1713, 1677, 1608, 1456, 1444, 1392, 1368, 1347, 1265 , 1252, 1154, 1103, 1078, 1041, 1031, 1001, 985, 888, 847, 814, 787, 773, 741, 717, 703, $969,619,605 \mathrm{~cm}^{-1}$; LRMS (ESI): Mass calcd for $\mathrm{C}_{16} \mathrm{H}_{20} \mathrm{NO}_{3}[\mathrm{M}+\mathrm{H}+\mathrm{MeCN}]^{+}:$274; found 274.<smiles>CC(=O)/C(=C/C=O)c1ccccc1</smiles>

(E)-4-oxo-3-phenylpent-2-enal: Prepared according to the general procedure using (Z)3-iodo-5,5-dimethoxypent-3-en-2-one $(0.500 \mathrm{~g}, 1.851 \mathrm{mmol})$ and phenylboronic acid ( $0.217 \mathrm{~g}, 2.22 \mathrm{mmol})$ to afford $0.135 \mathrm{~g}$ (42\% yield over 2 steps) of product as a yellow solid. Analytical data: ${ }^{1} \mathrm{H}$ NMR $\left(500 \mathrm{MHz}, \mathrm{CDCl}_{3}\right) \delta 9.64(\mathrm{~d}, \mathrm{~J}=7.6 \mathrm{~Hz}, 1 \mathrm{H}), 7.52-$ $7.40(\mathrm{~m}, 4 \mathrm{H}), 7.29-7.27(\mathrm{~m}, 1 \mathrm{H}), 6.73(\mathrm{~d}, \mathrm{~J}=7.6 \mathrm{~Hz}, 1 \mathrm{H}), 2.43(\mathrm{~s}, 3 \mathrm{H}) ;{ }^{13} \mathrm{C} \mathrm{NMR}(126$ $\left.\mathrm{MHz} \mathrm{CDCl}_{3}\right) \delta 199.8,194.2,155.5,132.9,132.1,130.2,129.8,128.7,28.1$; IR (film) 3045, 3007, 2929, 2881, 1683, 1665, 1632, 1608, 1441, 1421, 1369, 1336, 1319, 1228, 1168, 1111, 1078, 1024, 1017, 999, 985, 930, 898, 856, 776, 738, 707, 698, 663, 638, 614 $\mathrm{cm}^{-1}$; LRMS (ESI): Mass calcd for $\mathrm{C}_{11} \mathrm{H}_{11} \mathrm{O}_{2}[\mathrm{M}+\mathrm{H}]^{+}$: 175; found 175 . 


\section{Optimization Table for Enantioselective $\boldsymbol{\beta}$-Protonation}

Table 1. Optimization of reaction conditions.
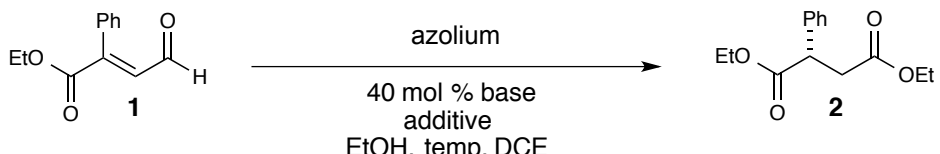

$\mathrm{EtOH}$, temp, DCE

\begin{tabular}{|c|c|c|c|c|c|c|c|c|c|}
\hline Entry & base & Additive (mol \%) & Temp. $\left({ }^{\circ} \mathrm{C}\right)$ & azolium (mol \%) & $\mathrm{EtOH}$ (equiv) & Time (h) & Conv. (\%) & yield (\%) & er \\
\hline 1 & $i-\mathrm{Pr}_{2} \mathrm{NEt}$ & - & 23 & $\mathbf{A}(10)$ & 10 & 12 & 100 & 80 & $66: 34$ \\
\hline 2 & $\mathrm{Et}_{3} \mathrm{~N}$ & - & 23 & A (10) & 10 & 10 & 100 & - & $64: 36$ \\
\hline 3 & TMEDA & - & 23 & A (10) & 10 & 8 & 100 & - & $60: 40$ \\
\hline 4 & $i-\mathrm{Pr}_{2} \mathrm{NEt}$ & $\mathrm{Mg}(\mathrm{OtBu})_{2}(20)$ & 23 & A (10) & 10 & 24 & 50 & - & $65: 35$ \\
\hline 5 & $i-\mathrm{Pr}_{2} \mathrm{NEt}$ & $\mathrm{Ti}(\mathrm{OiPr})_{4}(20)$ & 23 & $\mathbf{A}(10)$ & 10 & 16 & 100 & - & $70: 30$ \\
\hline 6 & $i-\mathrm{Pr}_{2} \mathrm{NEt}$ & $\mathrm{Sc}(\mathrm{OTf})_{3}(20)$ & 23 & A (10) & 10 & 18 & 100 & - & $64: 36$ \\
\hline 7 & $i-\mathrm{Pr}_{2} \mathrm{NEt}$ & $\mathrm{LiCl}(20)$ & 23 & A (10) & 10 & 12 & 100 & - & $80: 20$ \\
\hline 8 & $i-\mathrm{Pr}_{2}{ }_{2}^{\mathrm{NEt}}$ & HBD1 (20) & 23 & A (10) & 10 & 16 & 100 & - & $82: 18$ \\
\hline 9 & $i-\mathrm{Pr}_{2} \mathrm{NEt}$ & HBD1 (30) & 23 & A (10) & 10 & 12 & 100 & 44 & $88: 12$ \\
\hline 10 & $i-\mathrm{Pr}_{2}{ }_{2}^{\mathrm{NE}}$ & HBD1 (40) & 23 & A (10) & 10 & 6 & 100 & 33 & $90: 10$ \\
\hline 11 & $i-\mathrm{Pr}_{2} \mathrm{NEt}$ & HBD4 (30) & 23 & A (10) & 10 & 14 & 100 & - & $86: 14$ \\
\hline 12 & $i-\mathrm{Pr}_{2} \mathrm{NEt}$ & HBD5 (30) & 23 & $\mathbf{A}(10)$ & 10 & 18 & 100 & - & $82: 18$ \\
\hline 13 & $i-\mathrm{Pr}_{2} \mathrm{NEt}$ & HBD6 (30) & 23 & $\mathbf{A}(10)$ & 10 & 20 & 100 & - & $82: 18$ \\
\hline 14 & $i-\mathrm{Pr}_{2}{ }_{2}^{\mathrm{NEt}}$ & HBD7 (30) & 23 & A (10) & 10 & 4 & 100 & - & $64: 36$ \\
\hline 15 & $i-\mathrm{Pr}_{2} \mathrm{NEt}$ & HBD8 (30) & 23 & A (10) & 10 & 8 & 100 & - & $82: 18$ \\
\hline 16 & $i-\operatorname{Pr}_{2} \mathrm{NEt}$ & HBD9 (30) & 23 & $\mathbf{A}(10)$ & 10 & 8 & 100 & - & $82: 18$ \\
\hline 17 & $i-\mathrm{Pr}_{2}^{2} \mathrm{NEt}$ & HBD10 (30) & 23 & A (10) & 10 & 14 & 100 & - & $86: 14$ \\
\hline 18 & $i-\mathrm{Pr}_{2} \mathrm{NEt}$ & HBD11 (30) & 23 & A (10) & 10 & 12 & 100 & - & $85: 15$ \\
\hline 19 & $i-\mathrm{Pr}_{2} \mathrm{NEt}$ & HBD1 (30) & 0 & A (10) & 10 & 12 & 100 & 41 & $93: 7$ \\
\hline 20 & $i-\mathrm{Pr}_{2} \mathrm{NEt}$ & HBD1 (30), DMAP (10) & 0 & $\mathbf{A}(10)$ & 10 & 10 & 100 & 71 & $92: 8$ \\
\hline 21 & $i-\mathrm{Pr}_{2} \mathrm{NEt}$ & HBD1 (30), DMAP (10) & 0 & B (10) & 10 & - & - & 40 & $84: 16$ \\
\hline 22 & $i-\mathrm{Pr}_{2} \mathrm{NEt}$ & HBD1 (30), DMAP (10) & 0 & C (10) & 10 & - & - & 17 & $66: 34$ \\
\hline 23 & $i-\mathrm{Pr}_{2} \mathrm{NEt}$ & HBD1 (30), DMAP (10) & 0 & D (10) & 10 & - & - & 42 & 73:27 \\
\hline 24 & $i-\mathrm{Pr}_{2} \mathrm{NEt}$ & HBD1 (30), DMAP (10) & 0 & E (10) & 10 & - & - & 40 & $74: 26$ \\
\hline 25 & $i-\mathrm{Pr}_{2} \mathrm{NEt}$ & HBD2 (30), DMAP (10) & 0 & $\mathbf{A}(10)$ & 10 & 20 & 100 & 84 & $92: 8$ \\
\hline 26 & $i-\mathrm{Pr}_{2} \mathrm{NEt}$ & HBD3 (30), DMAP (10) & 0 & A (10) & 10 & 24 & 100 & 94 & $91: 9$ \\
\hline 27 & $i-\mathrm{Pr}_{2} \mathrm{NEt}$ & HBD3 (30) & 0 & $\mathbf{A}(10)$ & 10 & 36 & 100 & 81 & $96: 4$ \\
\hline 28 & $i-\mathrm{Pr}_{2} \mathrm{NEt}$ & HBD3 (30), DMAP (5) & 0 & A (10) & 10 & 24 & 100 & 85 & $94: 6$ \\
\hline 29 & $i-\mathrm{Pr}_{2} \mathrm{NEt}$ & HBD3 (30) & 0 & $\mathbf{A}(5)$ & 10 & 120 & 80 & 62 & $96: 4$ \\
\hline 30 & $i-\mathrm{Pr}_{2} \mathrm{NEt}$ & HBD3 (30), DMAP (5) & 0 & A (5) & 10 & 96 & 100 & 82 & $95: 5$ \\
\hline 31 & $i-\mathrm{Pr}_{2}^{2} \mathrm{NEt}$ & HBD3 (30) & 0 & $\mathbf{A}(10)$ & 7 & 40 & 100 & 79 & $96: 4$ \\
\hline 32 & $i-\mathrm{Pr}_{2} \mathrm{NEt}$ & HBD3 (30) & 0 & A (10) & 5 & 52 & 100 & 69 & $95: 5$ \\
\hline 33 & $i-\mathrm{Pr}_{2}{ }_{2}^{\mathrm{NEt}}$ & HBD3 (30) & 0 & $A(10)$ & 2 & 72 & 100 & 54 & $92: 8$ \\
\hline
\end{tabular}




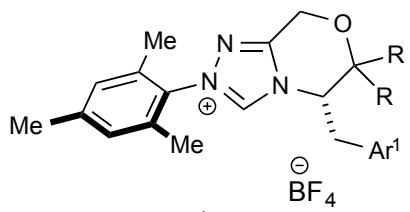

A, $\mathrm{R}=\mathrm{Me}, \mathrm{Ar}^{1}=\mathrm{Ph}$;

B, $\mathrm{R}=\mathrm{Ph}, \quad \mathrm{Ar}^{1}=\mathrm{Ph}$;

C, $\mathrm{R}=\mathrm{Ph}, \mathrm{Ar}^{1}=3$-indolyl<smiles></smiles>

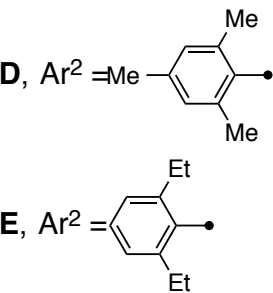<smiles>[X]C(Nc1cc(C(F)(F)F)cc(C(F)(F)F)c1)Nc1cc(C(F)(F)F)cc(C(F)(F)F)c1</smiles>

HBD1: $X=S$ HBD2: $X=O$<smiles>C=CC1CN2CCC1C2C(NC(=S)Nc1cc(C(F)(F)F)cc(C(F)(F)F)c1)c1ccnc2ccc(OC)cc12</smiles>

HBD5<smiles>CC1(C)OC(C(O)(c2ccccc2)c2ccccc2)[C@H](C(O)(c2ccccc2)c2ccccc2)O1</smiles>

HBD8<smiles>CN(C)c1ccncc1</smiles><smiles>C=CC1CN2CCC1C2[C@H](OCc1ccccc1)c1ccnc2ccc(NC(=S)Nc3cc(C(F)(F)F)cc(C(F)(F)F)c3)cc12</smiles>

HBD6<smiles>FC(F)(F)c1cc(NC(=S)N[C@H]2CCCC[C@@H]2OCc2ccccc2)cc(C(F)(F)F)c1</smiles>

HBD4 HBD3<smiles>O=P(O)(Oc1cc(C(F)(F)F)cc(C(F)(F)F)c1)Oc1c(-c2cc(C(F)(F)F)cc(C(F)(F)F)c2)cc2ccccc2c1-c1cc2ccccc2cc1-c1cc(C(F)(F)F)cc(C(F)(F)F)c1</smiles><smiles>CC1(C)OC(C(O)(c2ccccc2)c2ccccc2)[C@H](C(O)(c2ccccc2)c2ccccc2)O1</smiles>

HBD9<smiles>CN(C)[C@@H]1CCCC[C@H]1NC(=S)Nc1cc(C(F)(F)F)cc(C(F)(F)F)c1</smiles>

HBD10<smiles>CN(C)[C@H]1CCCC[C@H]1NC(=S)Nc1cc(C(F)(F)F)cc(C(F)(F)F)c1</smiles>

HBD11

\section{DMAP}




\section{General Procedure A for the Synthesis of Succinic Diester}

In a nitrogen filled dry box a screw-capped 2 dram vial equipped with a magnetic stirbar was charged with the corresponding enal $1(0.200 \mathrm{mmol})$, triazolium precatalyst $\mathbf{A}(0.1$ equiv), and HBD3 (0.30 equiv). The vial was capped with a septum cap, removed from the drybox and put under positive $\mathrm{N}_{2}$ pressure. The heterogeneous mixture was then diluted with degassed 1,2-dichloroethane $(4.0 \mathrm{~mL}, 0.05 \mathrm{M})$ stirred, and cooled to $0{ }^{\circ} \mathrm{C}$ in an ice bath. Ethanol (120 $\mu \mathrm{L}, 10$ equiv), water (1.8 $\mu \mathrm{L}, 5$ equiv), and Hünig's base (14 $\mu \mathrm{L}, 0.4$ equiv) were added sequentially via syringe. The reaction mixture was stirred at 0 ${ }^{\circ} \mathrm{C}$ for $36 \mathrm{~h}$ under static nitrogen pressure. Upon consumption of the aldehyde, monitored by NMR (all reactions were completed within 48 hours), the reaction mixture was concentrated in vасио. The material was purified by flash chromatography with EtOAc/hexanes to afford the corresponding succinic diester 2.

The corresponding racemic compounds were prepared by employing a similar protocol, except achiral triazolium precatalyst 2-mesityl-6,7-dihydro-5H-pyrrolo[2,1c] $[1,2,4]$ triazol-2-ium tetrafluoroborate $(10 \mathrm{~mol} \%)$ was used instead of $\mathbf{A}$, and the cocatalysts HBD3 and DMAP were not added.

\section{General Procedure B for the Synthesis of Succinic Diester}

In a nitrogen filled dry box a screw-capped 2 dram vial equipped with a magnetic stirbar was charged with the corresponding enal $1(0.200 \mathrm{mmol})$, triazolium precatalyst $\mathbf{A}(0.1$ equiv), and HBD3 (0.30 equiv). The vial was capped with a septum cap, removed from the drybox and put under positive $\mathrm{N}_{2}$ pressure. The heterogeneous mixture was then diluted with degassed 1,2-dichloroethane $(4.0 \mathrm{~mL}, 0.05 \mathrm{M})$ stirred, and cooled to $0{ }^{\circ} \mathrm{C}$ in an ice bath. Ethanol (120 $\mu \mathrm{L}, 10$ equiv), water $(1.8 \mu \mathrm{L}, 5$ equiv), DMAP (50 $\mu \mathrm{L}$ of $0.2 \mathrm{M}$ solution in 1,2-dichloroethane, 0.05 equiv) and Hünig's base (14 $\mu \mathrm{L}, 0.4$ equiv) was added sequentially via syringe. The reaction mixture was stirred at $0{ }^{\circ} \mathrm{C}$ for $24 \mathrm{~h}$ under static nitrogen pressure. Upon consumption of the aldehyde, monitored by ${ }^{1} \mathrm{H}$ NMR (all reactions were completed within 24 hours), the reaction mixture was concentrated in vacuo. The material was purified by flash chromatography with EtOAc/hexanes to afford the corresponding succinic diester 2.<smiles>CCOC(=O)C[C@H](C(=O)OCC)c1ccccc1</smiles>

Diethyl (S)-2-phenylsuccinate (2): Prepared according to the general procedure using (E)-ethyl 4-oxo-2-phenylbut-2-enoate $(0.041 \mathrm{~g}, 0.200 \mathrm{mmol})$ and purified by flash chromatography using $8 \%$ EtOAc/hexanes to afford $41 \mathrm{mg}$ ( $81 \%$ yield) of 2 as a colorless oil. Analytical data for 2: ${ }^{1} \mathrm{H}$ NMR $\left(500 \mathrm{MHz} \mathrm{CDCl}_{3}\right) \delta 7.36-7.26(\mathrm{~m}, 5 \mathrm{H}), 4.26-4.01$ $(\mathrm{m}, 5 \mathrm{H}), 3.18(\mathrm{dd}, \mathrm{J}=16.9,10.2 \mathrm{~Hz}, 1 \mathrm{H}), 2.65(\mathrm{dd}, \mathrm{J}=16.9,5.3 \mathrm{~Hz}, 1 \mathrm{H}), 1.21(\mathrm{dt}, \mathrm{J}=$ 13.0, 7.1 Hz, 6H); ${ }^{13} \mathrm{C}$ NMR $\left(125 \mathrm{MHz} ; \mathrm{CDCl}_{3}\right)$ : $\delta$ 173.1, 171.7, 138.0, 128.9(2C), 127.9(2C), 127.7, 61.3, 60.9, 47.4, 38.0, 14.3, 14.2; IR (film) 3089, 3064, 3032, 2982, 
2937, 2907, 2874, 1733, 1603, 1585, 1497, 1464, 1447, 1410, 1393, 1371, 1327, 1296, 1251, 1227, 1157, 1114, 1096, 1029, 856, 773, 731, $698 \mathrm{~cm}^{-1}$; LRMS (ESI): Mass calcd for $\mathrm{C}_{14} \mathrm{H}_{19} \mathrm{O}_{4}[\mathrm{M}+\mathrm{H}]^{+}:$251; found 251; $[\mathrm{a}]_{\mathrm{D}}{ }^{23}=+85.7\left(\mathrm{CHCl}_{3}, \mathrm{c}=1.19\right)^{4}$; Enantiomeric ratio was measured by chiral phase HPLC (Whelk-O, 0.5\% $i$-PrOH/Hexanes, 1.0 $\mathrm{mL} / \mathrm{min}, 230 \mathrm{~nm}$ ), Rt (major) $=34.0 \mathrm{~min}$, Rt $($ minor $)=31.7 \mathrm{~min}$; er $=96: 4$. Following general procedure B affords $42 \mathrm{mg}$ (85\% yield) $2,94: 6 \mathrm{er}$.

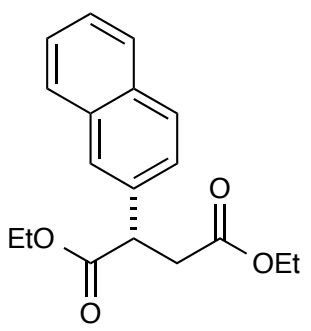

Diethyl (S)-2-(naphthalen-2-yl)succinate (3): Prepared according to the general procedure using (E)-ethyl 2-(naphthalen-2-yl)-4-oxobut-2-enoate $(0.051 \mathrm{~g}, 0.200 \mathrm{mmol})$ and purified by flash chromatography using $8 \% \mathrm{EtOAc} /$ hexanes to afford $46 \mathrm{mg}(76 \%$ yield) of 3 as a colorless oil. Analytical data for 3: ${ }^{1} \mathrm{H}$ NMR $\left(500 \mathrm{MHz}, \mathrm{CDCl}_{3}\right) \delta 7.84-$ $7.80(\mathrm{~m}, 3 \mathrm{H}), 7.76-7.74(\mathrm{~m}, 1 \mathrm{H}), 7.49-7.46(\mathrm{~m}, 2 \mathrm{H}), 7.42(\mathrm{dd}, \mathrm{J}=8.5,1.8 \mathrm{~Hz}, 1 \mathrm{H})$, $4.30-4.03(\mathrm{~m}, 5 \mathrm{H}), 3.29(\mathrm{dd}, \mathrm{J}=16.9,10.0 \mathrm{~Hz}, 1 \mathrm{H}), 2.75(\mathrm{dd}, \mathrm{J}=16.9,5.4 \mathrm{~Hz}, 1 \mathrm{H})$, $1.21(\mathrm{dt}, \mathrm{J}=15.6,7.1 \mathrm{~Hz}, 6 \mathrm{H}) ;{ }^{13} \mathrm{C} \mathrm{NMR}\left(126 \mathrm{MHz} \mathrm{CDCl}_{3}\right) \delta 173.1,171.7,135.4,133.5$, $132.8,128.7,128.0,127.8,126.8,126.4,126.2$, 125.8, 61.4, 60.9, 47.5, 38.1 , 14.3, 14.2; IR (film) 3058, 2982, 2936, 2907, 1730, 1373, 1330, 1268, 1221, 1155, 1096, 1029, 908, 859, 818, 731, $648 \mathrm{~cm}^{-1}$; LRMS (ESI): Mass calcd for $\mathrm{C}_{18} \mathrm{H}_{21} \mathrm{O}_{4}[\mathrm{M}+\mathrm{H}]^{+}:$301; found 301; Enantiomeric ratio was measured by chiral phase HPLC (Chiralcel ODH, $0.5 \%$ $\mathrm{PrOH} / \mathrm{Hexanes}, 1.0 \mathrm{~mL} / \mathrm{min}, 230 \mathrm{~nm}$ ), Rt (major) $=21.9 \mathrm{~min}$, Rt $($ minor $)=29.0 \mathrm{~min}$; er = 94:6.<smiles>CCOC(=O)C[C@H](C(=O)OCC)c1ccc([N+](=O)[O-])cc1</smiles>

Diethyl (S)-2-(p-tolyl)succinate (4): Prepared according to the general procedure using (E)-ethyl 4-oxo-2-(p-tolyl)but-2-enoate $(0.044 \mathrm{~g}, 0.200 \mathrm{mmol})$ and purified by flash chromatography using $8 \% \mathrm{EtOAc} /$ hexanes to afford $0.0392 \mathrm{~g}(74 \%)$ of 4 as a colorless oil. Analytical data for 4: ${ }^{1} \mathrm{H}$ NMR $\left(500 \mathrm{MHz}, \mathrm{CDCl}_{3}\right) \delta 7.20-7.10(\mathrm{~m}, 4 \mathrm{H}), 4.23-4.06$ $(\mathrm{m}, 4 \mathrm{H}), 4.02(\mathrm{dd}, \mathrm{J}=10.2,5.3 \mathrm{~Hz}, 1 \mathrm{H}), 3.16(\mathrm{dd}, \mathrm{J}=16.9,10.2 \mathrm{~Hz}, 1 \mathrm{H}), 2.63(\mathrm{dd}, \mathrm{J}=$ 16.9, $5.3 \mathrm{~Hz}, 1 \mathrm{H}), 2.32(\mathrm{~s}, 3 \mathrm{H}), 1.21(\mathrm{dt}, \mathrm{J}=14.3,7.1 \mathrm{~Hz}, 6 \mathrm{H}) ;{ }^{13} \mathrm{C}$ NMR $(126 \mathrm{MHz}$, $\left.\mathrm{CDCl}_{3}\right) \delta 173.1,171.7,135.4,133.5,132.8,128.7,128.0,127.8,126.8,126.4,126.2$, 125.8, 61.4, 60.9, 47.5, 38.1, 14.3, 14.2; IR (film) 2981, 2926, 1732, 1514, 1445, 1412, 1370, 1336, 1293, 1256, 1227, 1157, 1096, 1031, 856, 823, $791 \mathrm{~cm}^{-1}$; LRMS (ESI): Mass calcd for $\mathrm{C}_{15} \mathrm{H}_{21} \mathrm{O}_{4}[\mathrm{M}+\mathrm{H}]^{+}$: 265; found 265; Enantiomeric ratio was measured by chiral

\footnotetext{
${ }^{4}$ Y.-C. Chung, D. Janmanchi, H.-L. Wu, Org. Lett. 14, 2766-2769 (2012).
} 
phase HPLC (Chiralcel ODH, 1\% i-PrOH/Hexanes, $1.0 \mathrm{~mL} / \mathrm{min}, 210 \mathrm{~nm}$ ), Rt (major) = $6.96 \mathrm{~min}, \mathrm{Rt}$ (minor) $=8.59 \mathrm{~min}$; er $=91: 9$. Following general procedure $\mathrm{B}$ afforded $0.0435 \mathrm{~g}$ (82\% yield) of 4, 90:10 er.<smiles>CCOC(=O)C[C@H](C(=O)OCC)c1cccc(C)c1</smiles>

Diethyl (S)-2-(m-tolyl)succinate (5): Prepared according to the general procedure using (E)-ethyl 4-oxo-2-(m-tolyl)but-2-enoate $(0.044 \mathrm{~g}, 0.200 \mathrm{mmol})$ and purified by flash chromatography using $8 \%$ EtOAc/hexanes to afford $0.0408 \mathrm{~g}(77 \%)$ of 5 as a colorless oil. Analytical data for 5: ${ }^{1} \mathrm{H}$ NMR $\left(500 \mathrm{MHz}, \mathrm{CDCl}_{3}\right) \delta 7.20(\mathrm{t}, \mathrm{J}=7.5 \mathrm{~Hz}, 1 \mathrm{H}), 7.08(\mathrm{~d}$, $\mathrm{J}=8.1 \mathrm{~Hz}, 3 \mathrm{H}), 4.23-4.05(\mathrm{~m}, 4 \mathrm{H}), 4.02(\mathrm{dd}, \mathrm{J}=10.3,5.1 \mathrm{~Hz}, 1 \mathrm{H}), 3.17(\mathrm{dd}, \mathrm{J}=16.9$, $10.3 \mathrm{~Hz}, 1 \mathrm{H}), 2.63(\mathrm{dd}, \mathrm{J}=16.9,5.2 \mathrm{~Hz}, 1 \mathrm{H}), 2.33(\mathrm{~s}, 3 \mathrm{H}), 1.21(\mathrm{dt}, \mathrm{J}=11.8,7.1 \mathrm{~Hz}$, $6 \mathrm{H}) ;{ }^{13} \mathrm{C}$ NMR $\left(126 \mathrm{MHz}, \mathrm{CDCl}_{3}\right) \delta 173.2,171.7,138.6,137.9,128.8,128.5,128.4$, 124.9, 61.2, 60.8, 47.3, 38.1, 21.5, 14.3, 14.2; IR (film) 3450, 2981, 2934, 2905, 2872, $1742,1730,1607,1590,1491,1464,1445,1411,1370,1335,1297,1212$, 1096, 1031 , 858, 784, 735, $696 \mathrm{~cm}^{-1}$; LRMS (ESI): Mass calcd for $\mathrm{C}_{15} \mathrm{H}_{21} \mathrm{O}_{4}[\mathrm{M}+\mathrm{H}]^{+}: 265$; found 265; Enantiomeric ratio was measured by chiral phase HPLC (Chiralcel ODH, $0.5 \%$ $\mathrm{PrOH} / \mathrm{Hexanes}, 1.0 \mathrm{~mL} / \mathrm{min}, 210 \mathrm{~nm})$, Rt $($ major $)=13.9 \mathrm{~min}$, Rt $($ minor $)=19.3 \mathrm{~min}$; er $=$ 96:4.<smiles>CCOC(=O)C[C@H](C(=O)OCC)c1ccc(OC)cc1</smiles>

Diethyl (S)-2-(4-methoxyphenyl)succinate (6): Prepared according to the general procedure using (E)-ethyl 2-(4-methoxyphenyl)-4-oxobut-2-enoate (0.047 g, 0.200 mmol) and purified by flash chromatography using $20 \%$ EtOAc/hexanes to afford 0.0436 g $(78 \%)$ of 6 as a colorless oil. Analytical data for 6 : ${ }^{1} \mathrm{H}$ NMR $\left(500 \mathrm{MHz}, \mathrm{CDCl}_{3}\right) \delta 7.23$ - $7.18(\mathrm{~m}, 2 \mathrm{H}), 6.87-6.83(\mathrm{~m}, 2 \mathrm{H}), 4.12$ (dddd, $\mathrm{J}=21.1,14.0,10.8,7.1 \mathrm{~Hz}, 4 \mathrm{H}), 4.01$ $(\mathrm{dd}, \mathrm{J}=10.0,5.5 \mathrm{~Hz}, 1 \mathrm{H}), 3.79(\mathrm{~s}, 3 \mathrm{H}), 3.15(\mathrm{dd}, \mathrm{J}=16.8,10.1 \mathrm{~Hz}, 1 \mathrm{H}), 2.63$ (dd, J = $16.8,5.5 \mathrm{~Hz}, 1 \mathrm{H}), 1.21(\mathrm{dt}, \mathrm{J}=12.9,7.1 \mathrm{~Hz}, 6 \mathrm{H}) ;{ }^{13} \mathrm{C} \mathrm{NMR}\left(126 \mathrm{MHz}, \mathrm{CDCl}_{3}\right) \delta 173.2$, 171.6, 159.0, 129.9, 128.8, 114.2, 61.0, 60.7, 55.3, 46.5, 38.0, 14.2, 14.1; IR (film) 2982, 2936, 2908, 2837, 1726, 1611, 1584, 1512, 1464, 1443, 1410, 1393, 1371, 1302, 1248, 1177, 1159, 1112, 1096, 1030, 906, 856, 833, 794, 727, $648 \mathrm{~cm}^{-1}$; LRMS (ESI): Mass calcd for $\mathrm{C}_{15} \mathrm{H}_{21} \mathrm{O}_{5}[\mathrm{M}+\mathrm{H}]^{+}: 281$; found 281; Enantiomeric ratio was measured by chiral phase HPLC (Chiralcel IA, 5\% $i$-PrOH/Hexanes, $1.0 \mathrm{~mL} / \mathrm{min}, 210 \mathrm{~nm}$ ), Rt (major) $=10.2$ $\min , \mathrm{Rt}($ minor $)=9.5 \mathrm{~min}$; er $=92: 8$. 
<smiles>CCOC(=O)C[C@H](C(=O)OCC)c1ccc(OC)c(OC)c1</smiles>

Diethyl (S)-2-(3,4-dimethoxyphenyl)succinate (7): Prepared according to the general procedure using (E)-ethyl 2-(3,4-dimethoxyphenyl)-4-oxobut-2-enoate (0.053 g, 0.200 mmol) and purified by flash chromatography using $20 \%$ EtOAc/hexanes to afford 0.0441 $\mathrm{g}(71 \%)$ of 7 as a colorless oil. Analytical data for $7:{ }^{1} \mathrm{H} \mathrm{NMR}\left(500 \mathrm{MHz}, \mathrm{CDCl}_{3}\right) \delta 6.82$ $(\mathrm{dd}, \mathrm{J}=6.8,2.8 \mathrm{~Hz}, 3 \mathrm{H}), 4.21-4.08(\mathrm{~m}, 4 \mathrm{H}), 4.00(\mathrm{dd}, \mathrm{J}=10.0,5.4 \mathrm{~Hz}, 1 \mathrm{H}), 3.87(\mathrm{~s}$, $3 \mathrm{H}), 3.86(\mathrm{~s}, 3 \mathrm{H}), 3.16(\mathrm{dd}, \mathrm{J}=16.8,10.1 \mathrm{~Hz}, 1 \mathrm{H}), 2.65(\mathrm{dd}, \mathrm{J}=16.8,5.4 \mathrm{~Hz}, 1 \mathrm{H}), 1.22$ $(\mathrm{dt}, \mathrm{J}=11.1,7.1 \mathrm{~Hz}, 6 \mathrm{H}) ;{ }^{13} \mathrm{C} \mathrm{NMR}\left(126 \mathrm{MHz}, \mathrm{CDCl}_{3}\right) \delta$ 173.3, 171.7, 149.2, 148.6, $130.5,120.1,111.4,110.9,61.2,60.9,56.0,47.0,38.2,31.1,14.3,14.2$; IR (film) 2981, 2962, 2937, 2908, 2874, 2837, 1731, 1592, 1516, 1465, 1420, 1393, 1371, 1328, 1295 , 1261, 1243, 1174, 1152, 1096, 1028, 914, 858, 808, 765, 731, $647 \mathrm{~cm}^{-1}$; LRMS (ESI): Mass calcd for $\mathrm{C}_{16} \mathrm{H}_{23} \mathrm{O}_{6}[\mathrm{M}+\mathrm{H}]^{+}$: 311; found 311; Enantiomeric ratio was measured by chiral phase HPLC (Chiralcel ADH, 3\% $i$-PrOH/Hexanes, $1.0 \mathrm{~mL} / \mathrm{min}, 210 \mathrm{~nm}$ ), Rt (major) $=28.3 \mathrm{~min}, \mathrm{Rt}($ minor $)=24.2 \mathrm{~min}$; er $=93: 7$. Following general procedure $\mathrm{B}$ afforded $0.0578 \mathrm{~g}$ (93\% yield) of 7, er $=90: 10$.<smiles>CCOC(=O)C[C@H](C(=O)OCC)c1ccc(F)cc1</smiles>

Diethyl (S)-2-(4-fluorophenyl)succinate (8): Prepared according to the general procedure using (E)-ethyl 2-(4-fluorophenyl)-4-oxobut-2-enoate $(0.044 \mathrm{~g}, 0.200 \mathrm{mmol})$ and purified by flash chromatography using $8 \%$ EtOAc/hexanes to afford $0.0404 \mathrm{~g}(75 \%)$ of 8 as a colorless oil. Analytical data for 8: ${ }^{1} \mathrm{H} \mathrm{NMR}\left(500 \mathrm{MHz} ; \mathrm{CDCl}_{3}\right)$ : $\delta 7.30-7.22$ $(\mathrm{m}, 2 \mathrm{H}), 7.04-6.97(\mathrm{~m}, 2 \mathrm{H}), 4.22-4.00(\mathrm{~m}, 5 \mathrm{H}), 3.15(\mathrm{ap} \mathrm{dd}, J=16.8,9.8 \mathrm{~Hz}, 1 \mathrm{H})$, 2.64 (ap dd, $J=16.9,5.7 \mathrm{~Hz}, 1 \mathrm{H}), 1.22$ (t, $J=7.1 \mathrm{~Hz}, 3 \mathrm{H}), 1.20(\mathrm{t}, J=7.1 \mathrm{~Hz}, 3 \mathrm{H}) ;{ }^{13} \mathrm{C}$ NMR $\left(125 \mathrm{MHz} ; \mathrm{CDCl}_{3}\right): \delta 172.8,171.3,162.2\left(\mathrm{~d}, J_{\mathrm{C}-\mathrm{F}}=246.1 \mathrm{~Hz}\right), 133.6\left(\mathrm{~d}, J_{\mathrm{C}-\mathrm{F}}=3.3\right.$ $\mathrm{Hz}), 129.4(2 \mathrm{C}, \mathrm{d}, J=8.1 \mathrm{~Hz}), 115.7\left(2 \mathrm{C}, \mathrm{d}, J_{\mathrm{C}-\mathrm{F}}=21.5 \mathrm{~Hz}\right), 61.3,60.8,46.5,37.9,14.2$, 14.1; ${ }^{19} \mathrm{~F} \mathrm{NMR} \mathrm{(376} \mathrm{MHz;} \mathrm{CDCl}_{3}$ ): $\delta-114.9$ (m); IR (film) 2983, 2939, 2908, 2875, 1734, 1606, 1466, 1447, 1412, 1393, 1372, 1334, 1295, 1226, 1158, 1098, 1030, 841, 811 $\mathrm{cm}^{-1}$; LRMS (ESI): Mass calcd for $\mathrm{C}_{14} \mathrm{H}_{18} \mathrm{O}_{4} \mathrm{~F}[\mathrm{M}+\mathrm{H}]^{+}:$269; found 269; Enantiomeric ratio was measured by chiral phase HPLC (Chiralcel IA, $1 \% i$-PrOH/Hexanes, 1.0 $\mathrm{mL} / \mathrm{min}, 210 \mathrm{~nm}$ ), Rt (major) $=16.6 \mathrm{~min}$, Rt $($ minor $)=12.1 \mathrm{~min}$; er = 93:7. 


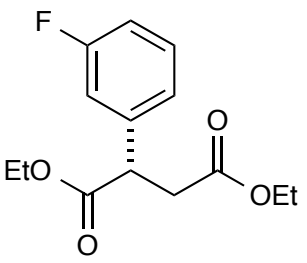

Diethyl (S)-2-(3-fluorophenyl)succinate (9): Prepared according to the general procedure using (E)-ethyl 2-(3-fluorophenyl)-4-oxobut-2-enoate $(28 \mathrm{mg}, 0.126 \mathrm{mmol})$ and purified by flash chromatography using $8 \%$ EtOAc/hexanes to afford $18.1 \mathrm{mg} \mathrm{(54 \%}$ yield) of 9 as a colorless oil. Analytical data for 9: ${ }^{1} \mathrm{H}$ NMR (500 MHz; $\left.\mathrm{CDCl}_{3}\right)$ : $\delta 7.32$ (ap td, $J=8.0,6.0 \mathrm{~Hz}, 1 \mathrm{H}), 7.09$ (ap dt, $J=7.7,1.2 \mathrm{~Hz}, 1 \mathrm{H}), 7.06-6.97(\mathrm{~m}, 2 \mathrm{H}), 4.34-$ 3.99 (m, 5H), 3.19 (ap dd, $J=16.9,9.9 \mathrm{~Hz}, 1 \mathrm{H}$ ), 2.68 (ap dd, $J=16.9,5.5 \mathrm{~Hz}, 1 \mathrm{H}$ ), 1.25 $(\mathrm{t}, J=7.2 \mathrm{~Hz}, 3 \mathrm{H}), 1.24(\mathrm{t}, J=7.1 \mathrm{~Hz}, 3 \mathrm{H}) ;{ }^{13} \mathrm{C} \mathrm{NMR}\left(125 \mathrm{MHz} ; \mathrm{CDCl}_{3}\right): \delta 172.6,171.4$, $163.0\left(\mathrm{~d}, J_{\mathrm{C}-\mathrm{F}}=246.7 \mathrm{~Hz}\right), 140.3\left(\mathrm{~d}, J_{\mathrm{C}-\mathrm{F}}=7.4 \mathrm{~Hz}\right), 130.4\left(\mathrm{~d}, J_{\mathrm{C}-\mathrm{F}}=8.3 \mathrm{~Hz}\right), 123.6\left(\mathrm{~d}, J_{\mathrm{C}-\mathrm{F}}\right.$ $=2.9 \mathrm{~Hz}), 115.0\left(\mathrm{~d}, J_{\mathrm{C}-\mathrm{F}}=22.0 \mathrm{~Hz}\right), 114.7\left(\mathrm{~d}, J_{\mathrm{C}-\mathrm{F}}=21.0 \mathrm{~Hz}\right), 61.5,61.0,47.1,37.8,37.8$, 14.3, 14.2; ${ }^{19} \mathrm{~F}$ NMR (376 MHz; $\mathrm{CDCl}_{3}$ ): $\delta$-112.3 (m); IR (film) 3067, 2983, 2938, 2908, 2875, 1733, 1615, 1591, 1489, 1449, 1411, 1393, 1372, 1336, 1298, 1263, 1240, 1217, 1176, 1160, 1096, 1030, 966, 941, 899, 873, 861, 786, $688 \mathrm{~cm}^{-1}$; LRMS (ESI): Mass calcd for $\mathrm{C}_{14} \mathrm{H}_{18} \mathrm{O}_{4} \mathrm{~F}[\mathrm{M}+\mathrm{H}]^{+}$: 269; found 269; Enantiomeric ratio was measured by chiral phase HPLC (Chiralcel ODH, 1\% $i$-PrOH/Hexanes, $1.0 \mathrm{~mL} / \mathrm{min}, 210 \mathrm{~nm})$, Rt (major) $=$ $8.26 \mathrm{~min}$, Rt $($ minor $)=18.4 \mathrm{~min}$; er $=93: 7$. Following general procedure $\mathrm{B}$ afforded $0.0234 \mathrm{~g}(69 \%$ yield $)$ of $\mathbf{9}$, er $=92: 8$.<smiles>CCOC(=O)C[C@H](C(=O)OCC)c1ccccc1F</smiles>

Diethyl (S)-2-(2-fluorophenyl)succinate (10): Prepared according to the general procedure using (E)-ethyl 2-(2-fluorophenyl)-4-oxobut-2-enoate $(0.044 \mathrm{~g}, 0.200 \mathrm{mmol})$ and purified by flash chromatography using $8 \%$ EtOAc/hexanes to afford $0.0334 \mathrm{~g} \mathrm{(62 \% )}$ of $\mathbf{1 0}$ as a colorless oil. Analytical data for 10: ${ }^{1} \mathrm{H}$ NMR $\left(500 \mathrm{MHz}, \mathrm{CDCl}_{3}\right) \delta 7.27$ (dd, J $=3.5,1.8 \mathrm{~Hz}, 1 \mathrm{H}), 7.26-7.23(\mathrm{~m}, 1 \mathrm{H}), 7.13-7.03(\mathrm{~m}, 2 \mathrm{H}), 4.38(\mathrm{dd}, \mathrm{J}=9.5,5.7 \mathrm{~Hz}$, $1 \mathrm{H}), 4.20-4.10(\mathrm{~m}, 4 \mathrm{H}), 3.17(\mathrm{dd}, \mathrm{J}=16.8,9.5 \mathrm{~Hz}, 1 \mathrm{H}), 2.65(\mathrm{dd}, \mathrm{J}=16.8,5.7 \mathrm{~Hz}, 1 \mathrm{H})$, $1.21(\mathrm{dt}, \mathrm{J}=11.7,7.1 \mathrm{~Hz}, 6 \mathrm{H}) ;{ }^{13} \mathrm{C}$ NMR $\left(126 \mathrm{MHz}, \mathrm{CDCl}_{3}\right) \delta$ 172.4, 171.5, 161.5, 159.5, 129.4, 129.4, 129.3, 125.5, 125.4, 124.5, 124.5, 116.0, 115.8, 61.5, 60.9, 40.8, 40.8, 37.0, 14.3, 14.2; IR (film) 2983, 2937, 1735, 1493, 1458, 1371, 130, 1301, 1233, 1161, 1096, 1029, $759 \mathrm{~cm}^{-1}$; LRMS (ESI): Mass calcd for $\mathrm{C}_{14} \mathrm{H}_{18} \mathrm{FO}_{4}[\mathrm{M}+\mathrm{H}]^{+}$: 269; found 269; Enantiomeric ratio was measured by chiral phase HPLC (Chiralcel ODH, $1 \% i$ $\mathrm{PrOH} / \mathrm{Hexanes}, 1.0 \mathrm{~mL} / \mathrm{min}, 210 \mathrm{~nm}$ ), Rt (major) $=8.88 \mathrm{~min}, \mathrm{Rt}($ minor $)=24.3 \mathrm{~min}$; $\mathrm{er}=$ 89:11. Following general procedure B afforded $0.0403 \mathrm{~g}$ (75\% yield) of 10, er $=87: 13$. 


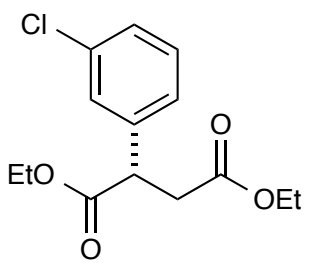

Diethyl (S)-2-(3-chlorophenyl)succinate (11): Prepared according to the general procedure using (E)-ethyl 2-(3-chlorophenyl)-4-oxobut-2-enoate $(0.048 \mathrm{~g}, 0.200 \mathrm{mmol})$ and purified by flash chromatography using $8 \%$ EtOAc/hexanes to afford $0.0401 \mathrm{~g} \mathrm{(70 \% )}$ of $\mathbf{1 1}$ as a colorless oil. Analytical data for 11: ${ }^{1} \mathrm{H}$ NMR $\left(500 \mathrm{MHz}, \mathrm{CDCl}_{3}\right) \delta 7.29(\mathrm{~m}$, $1 \mathrm{H}), 7.25(\mathrm{~m}, 2 \mathrm{H}), 7.20-7.15(\mathrm{~m}, 1 \mathrm{H}), 4.24-4.06(\mathrm{~m}, 4 \mathrm{H}), 4.03(\mathrm{dd}, \mathrm{J}=9.9,5.5 \mathrm{~Hz}$, $1 \mathrm{H}), 3.16(\mathrm{dd}, \mathrm{J}=16.9,9.9 \mathrm{~Hz}, 1 \mathrm{H}), 2.65(\mathrm{dd}, \mathrm{J}=16.9,5.5 \mathrm{~Hz}, 1 \mathrm{H}), 1.22(\mathrm{q}, \mathrm{J}=7.3 \mathrm{~Hz}$, $6 \mathrm{H}) ;{ }^{13} \mathrm{C}$ NMR $\left(126 \mathrm{MHz}, \mathrm{CDCl}_{3}\right) \delta 172.5,171.3,139.8,134.7,130.2,128.1,128.0$, 126.1, 61.5, 61.0, 47.1, 37.8, 14.3, 14.2; IR (film) 3066, 2982, 2937, 2907, 2873, 1733, 1596, 1574, 1477, 1432, 1410, 1393, 1370, 1334, 1293, 1250, 1229, 1174, 1159, 1095, $1082,1000,856,788,771,686 \mathrm{~cm}^{-1}$; LRMS (ESI): Mass calcd for $\mathrm{C}_{14} \mathrm{H}_{18} \mathrm{ClO}_{4}[\mathrm{M}+\mathrm{H}]^{+}$: 285; found 285; Enantiomeric ratio was measured by chiral phase HPLC (Chiralcel $\mathrm{ODH}, 1 \% i-\mathrm{PrOH} / \mathrm{Hexanes}, 1.0 \mathrm{~mL} / \mathrm{min}, 230 \mathrm{~nm})$, Rt (major) $=8.11 \mathrm{~min}$, Rt $($ minor $)=$ $18.3 \mathrm{~min}$; er $=93: 7$.<smiles>CCOC(=O)C[C@H](C(=O)OCC)c1cccc([N+](=O)[O-])c1</smiles>

Diethyl (S)-2-(3-nitrophenyl)succinate (12): Prepared according to the general procedure using (E)-ethyl 2-(3-nitrophenyl)-4-oxobut-2-enoate $(0.050 \mathrm{~g}, 0.200 \mathrm{mmol})$ and purified by flash chromatography using $20 \%$ EtOAc/hexanes to afford $0.0339 \mathrm{~g}$ (57\%) of $\mathbf{1 2}$ as a colorless oil. Analytical data for 12 : ${ }^{1} \mathrm{H}$ NMR $\left(500 \mathrm{MHz}, \mathrm{CDCl}_{3}\right) \delta 8.18$ $(\mathrm{t}, \mathrm{J}=1.9 \mathrm{~Hz}, 1 \mathrm{H}), 8.15(\mathrm{ddd}, \mathrm{J}=8.1,2.1,0.9 \mathrm{~Hz}, 1 \mathrm{H}), 7.65(\mathrm{~d}, \mathrm{~J}=7.7 \mathrm{~Hz}, 1 \mathrm{H}), 7.52(\mathrm{t}, \mathrm{J}$ $=7.9 \mathrm{~Hz}, 1 \mathrm{H}), 4.23-4.09(\mathrm{~m}, 5 \mathrm{H}), 3.22(\mathrm{dd}, \mathrm{J}=16.9,9.3 \mathrm{~Hz}, 1 \mathrm{H}), 2.73(\mathrm{dd}, \mathrm{J}=16.9,6.1$ $\mathrm{Hz}, 1 \mathrm{H}), 1.26-1.19(\mathrm{~m}, 6 \mathrm{H}) ;{ }^{13} \mathrm{C}$ NMR $\left(126 \mathrm{MHz}, \mathrm{CDCl}_{3}\right) \delta 171.9,171.0,148.6,139.9$, 134.2, 129.9, 123.1, 122.9, 61.8, 61.2, 47.0, 37.6, 14.3, 14.2; IR (film) 3448, 3090, 2982, 2938, 1733, 1532, 1477, 1465, 1445, 1410, 1394, 1370, 1351, 1301, 1255, 1230, 1176, 1160, 1097, 1028, 736, $681 \mathrm{~cm}^{-1}$; LRMS (ESI): Mass calcd for $\mathrm{C}_{14} \mathrm{H}_{18} \mathrm{NO}_{6}[\mathrm{M}+\mathrm{H}]^{+}:$: 296; found 296; Enantiomeric ratio was measured by chiral phase HPLC (Chiralcel ODH, 3\% $i$-PrOH/Hexanes, $1.0 \mathrm{~mL} / \mathrm{min}, 210 \mathrm{~nm}$ ), Rt (major) $=25.0 \mathrm{~min}, \mathrm{Rt}$ (minor) $=12.5 \mathrm{~min}$; ee $=89: 11$. Following general procedure B afforded $0.0401 \mathrm{~g}(68 \%$ yield $)$ of $\mathbf{1 2}$, er $=83: 17$.<smiles>CCOC(=O)CC(C(=O)OCC)c1cccs1</smiles>

Diethyl (R)-2-(thiophen-2-yl)succinate (13): Prepared according to the general procedure using (Z)-ethyl 4-oxo-2-(thiophen-2-yl)but-2-enoate $(0.042 \mathrm{~g}, 0.200 \mathrm{mmol})$ and purified by flash chromatography using $10 \%$ EtOAc/hexanes to afford $0.0315 \mathrm{~g}$ 
(61\%) of 13 as a clear yellow oil. Analytical data for 13: ${ }^{1} \mathrm{H}$ NMR $\left(500 \mathrm{MHz}, \mathrm{CDCl}_{3}\right) \delta$ $7.21(\mathrm{dd}, \mathrm{J}=4.5,1.8 \mathrm{~Hz}, 1 \mathrm{H}), 6.95(\mathrm{~d}, \mathrm{~J}=4.7 \mathrm{~Hz}, 2 \mathrm{H}), 4.35(\mathrm{dd}, \mathrm{J}=10.0,5.3 \mathrm{~Hz}, 1 \mathrm{H})$, $4.25-4.09(\mathrm{~m}, 4 \mathrm{H}), 3.21(\mathrm{dd}, \mathrm{J}=16.8,10.0 \mathrm{~Hz}, 1 \mathrm{H}), 2.78(\mathrm{dd}, \mathrm{J}=16.8,5.3 \mathrm{~Hz}, 1 \mathrm{H})$, $1.24(\mathrm{t}, \mathrm{J}=7.1 \mathrm{~Hz}, 6 \mathrm{H}) ;{ }^{13} \mathrm{C} \mathrm{NMR}\left(126 \mathrm{MHz}, \mathrm{CDCl}_{3}\right) \delta 172.1,171.2,139.9,127.0,125.6$, 125.0, 61.6, 61.0, 42.6, 38.5, 14.3, 14.2; IR (film) 3108, 2982, 2936, 1732, 1465, 1445 , $1409,1393,1373,1349,1329,1256,1228,1174,1160,1115,1096,1028,854,702 \mathrm{~cm}^{-1}$; LRMS (ESI): Mass calcd for $\mathrm{C}_{12} \mathrm{H}_{17} \mathrm{O}_{4} \mathrm{~S}[\mathrm{M}+\mathrm{H}]^{+}:$257; found 257; Enantiomeric ratio was measured by chiral phase HPLC (Chiralcel ODH, 1\% $i$-PrOH/Hexanes, $1.0 \mathrm{~mL} / \mathrm{min}, 230$ $\mathrm{nm})$, Rt (major) $=14.5 \mathrm{~min}$, Rt (minor) $=34.1 \mathrm{~min}$; er $=95: 5$. Following general procedure B afforded $0.0411 \mathrm{~g}(80 \%$ yield $)$ of 12 , er $=77: 23$.

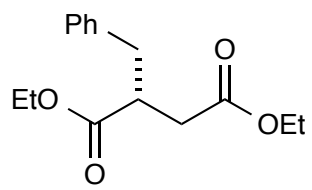

Ethyl (E)-2-benzyl-4-oxobut-2-enoate (14): Prepared according to the general procedure using (E)-ethyl 2-benzyl-4-oxobut-2-enoate $(0.044 \mathrm{~g}, 0.200 \mathrm{mmol})$ and purified by flash chromatography using $8 \%$ EtOAc/hexanes to afford $23 \mathrm{mg}$ (44\% yield) of 14 as a colorless oil. Analytical data for 14: ${ }^{1} \mathrm{H} 1 \mathrm{H} \mathrm{NMR}\left(500 \mathrm{MHz}, \mathrm{CDCl}_{3}\right) \delta 7.29(\mathrm{~d}$, $\mathrm{J}=7.1 \mathrm{~Hz}, 1 \mathrm{H}), 7.24-7.19(\mathrm{~m}, 2 \mathrm{H}), 7.17(\mathrm{~d}, \mathrm{~J}=7.0 \mathrm{~Hz}, 2 \mathrm{H}), 4.10(\mathrm{dq}, \mathrm{J}=10.8,7.1 \mathrm{~Hz}$, $4 \mathrm{H}), 3.13-3.07(\mathrm{~m}, 1 \mathrm{H}), 3.04(\mathrm{dd}, \mathrm{J}=13.5,6.5 \mathrm{~Hz}, 1 \mathrm{H}), 2.76(\mathrm{dd}, \mathrm{J}=13.5,8.2 \mathrm{~Hz}, 1 \mathrm{H})$, $2.66(\mathrm{dd}, \mathrm{J}=16.7,9.2 \mathrm{~Hz}, 1 \mathrm{H}), 2.39(\mathrm{dd}, \mathrm{J}=16.7,5.0 \mathrm{~Hz}, 1 \mathrm{H}), 1.21(\mathrm{dt}, \mathrm{J}=16.6,7.1 \mathrm{~Hz}$, $6 \mathrm{H}) ;{ }^{13} \mathrm{C} \mathrm{NMR}\left(126 \mathrm{MHz}, \mathrm{CDCl}_{3}\right) \delta 174.4,172.0,138.4,129.2,128.6,126.8,60.8,60.7$, 43.3, 38.0, 35.4, 14.3, 14.2; IR (film) 3028, 2980, 2930, 1728, 1496, 1454, 1446, 1393 , $1371,1348,1327,1256,1176,1158,1096,1076,1030,971,856,743,699 \mathrm{~cm}^{-1}$; LRMS (ESI): Mass calcd for $\mathrm{C}_{15} \mathrm{H}_{21} \mathrm{O}_{4}[\mathrm{M}+\mathrm{H}]^{+}$: 265; found 265; Enantiomeric ratio was measured by chiral phase HPLC (Chiralcel ODH, $1 \% i$-PrOH/Hexanes, $1.0 \mathrm{~mL} / \mathrm{min}, 210$ $\mathrm{nm})$, Rt (major) $=12.6 \mathrm{~min}$, Rt (minor) $=18.1 \mathrm{~min}$; er $=92: 8$. Following general procedure B afforded $0.0302 \mathrm{~g}(57 \%$ yield $)$ of 14 , er $=85: 15$.

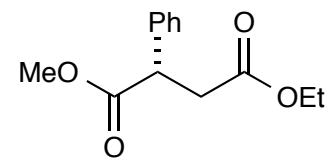

4-ethyl 1-methyl (S)-2-phenylsuccinate (15): Prepared according to the general procedure using (E)-methyl 4-oxo-2-phenylbut-2-enoate $(0.038 \mathrm{~g}, 0.200 \mathrm{mmol})$ and purified by flash chromatography using $8 \%$ EtOAc/hexanes to afford $0.0339 \mathrm{~g}(72 \%)$ of 15 as a colorless oil. Analytical data for 15: ${ }^{1} \mathrm{H}$ NMR $\left(500 \mathrm{MHz}, \mathrm{CDCl}_{3}\right) \delta 7.35-7.27$ $(\mathrm{m}, 5 \mathrm{H}), 4.15-4.11(\mathrm{~m}, 2 \mathrm{H}), 4.10-4.07(\mathrm{~m}, 1 \mathrm{H}), 3.68(\mathrm{~s}, 3 \mathrm{H}), 3.20(\mathrm{dd}, \mathrm{J}=16.9,10.2$ $\mathrm{Hz}, 1 \mathrm{H}), 2.66(\mathrm{dd}, \mathrm{J}=16.9,5.3 \mathrm{~Hz}, 1 \mathrm{H}), 1.23(\mathrm{t}, \mathrm{J}=7.1 \mathrm{~Hz}, 3 \mathrm{H}) ;{ }^{13} \mathrm{C}$ NMR $(126 \mathrm{MHz}$, $\left.\mathrm{CDCl}_{3}\right) \delta 173.6,171.6,137.8,129.0,127.9,127.8,60.9,52.5,47.3,38.1,14.3$; LRMS (ESI): Mass calcd for $\mathrm{C}_{13} \mathrm{H}_{17} \mathrm{O}_{4}[\mathrm{M}+\mathrm{H}]^{+}$: 237; found 237; Enantiomeric ratio was measured by chiral phase HPLC (Chiralcel Whelk-O, 0.5\% $i$-PrOH/Hexanes, 1.0 $\mathrm{mL} / \mathrm{min}, 210 \mathrm{~nm})$, Rt (major) $=36.0 \mathrm{~min}, \mathrm{Rt}($ minor $)=10.2 \mathrm{~min}$; er $=90: 10$. 
<smiles>CCOC(=O)C[C@H](C(=O)OCc1ccccc1)c1ccccc1</smiles>

1-benzyl 4-ethyl (S)-2-phenylsuccinate (16): Prepared according to the general procedure using (E)-benzyl 4-oxo-2-phenylbut-2-enoate $(0.053 \mathrm{~g}, 0.200 \mathrm{mmol})$ and purified by flash chromatography using $8 \%$ EtOAc/hexanes to afford $0.0432 \mathrm{~g}$ (69\%)of 16 as a colorless oil. Analytical data for 16: ${ }^{1} \mathrm{H}$ NMR $\left(500 \mathrm{MHz}, \mathrm{CDCl}_{3}\right) \delta 7.29$ (q, J = $5.7 \mathrm{~Hz}, 8 \mathrm{H}), 7.21(\mathrm{dd}, \mathrm{J}=7.2,2.0 \mathrm{~Hz}, 2 \mathrm{H}), 5.12(\mathrm{q}, \mathrm{J}=12.5 \mathrm{~Hz}, 2 \mathrm{H}), 4.15(\mathrm{dd}, \mathrm{J}=10.1$, $5.3 \mathrm{~Hz}, 1 \mathrm{H}), 4.10(\mathrm{qd}, \mathrm{J}=7.1,1.8 \mathrm{~Hz}, 2 \mathrm{H}), 3.21(\mathrm{dd}, \mathrm{J}=16.9,10.1 \mathrm{~Hz}, 1 \mathrm{H}), 2.69(\mathrm{dd}, \mathrm{J}=$ $16.9,5.4 \mathrm{~Hz}, 1 \mathrm{H}), 1.19$ (t, J = 7.1 Hz, 3H); ${ }^{13} \mathrm{C} \mathrm{NMR}(126 \mathrm{MHz}, \mathrm{CDCl} 3) \delta 172.9,171.6$, $137.7,135.9,129.0,128.6,128.2,128.0,127.9,127.8,66.9,60.9,47.4,37.9$, 14.2; IR (film) 3065, 3033, 2980, 2918,2849, 1732, 1652, 1635, 1602, 1576, 1558, 1540, 1506, 1497, 1472, 1455, 1409, 1373, 1329, 1293, 1279, 1250, 1221, 1153, 1095, 1025, 913, $850,774,733,696,656,603 \mathrm{~cm}^{-1}$; LRMS (ESI): Mass calcd for $\mathrm{C}_{19} \mathrm{H}_{21} \mathrm{O}_{4}[\mathrm{M}+\mathrm{H}]^{+}: 313$; found 313; Enantiomeric ratio was measured by chiral phase HPLC (Chiralcel Whelk-O, $0.5 \% \mathrm{i}-\mathrm{PrOH} / \mathrm{Hexanes}, 1.0 \mathrm{~mL} / \mathrm{min}, 210 \mathrm{~nm})$, Rt $($ major $)=61.2 \mathrm{~min}$, Rt $($ minor $)=55.2$ $\min$; er $=95: 5$.

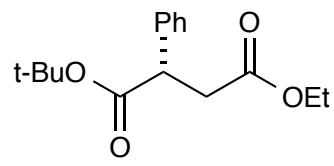

1-(tert-butyl) 4-ethyl (S)-2-phenylsuccinate (17): Prepared according to the general procedure using (E)-tert-butyl 4-oxo-2-phenylbut-2-enoate $(0.046 \mathrm{~g}, 0.200 \mathrm{mmol})$ and purified by flash chromatography using $8 \% \mathrm{EtOAc/hexanes} \mathrm{to} \mathrm{afford} 0.046 \mathrm{~g}$ (83\%) of 17 as a colorless oil. Analytical data for 17: ${ }^{1} \mathrm{H} \mathrm{NMR}\left(500 \mathrm{MHz}, \mathrm{CDCl}_{3}\right) \delta 7.32-7.27(\mathrm{~m}$, $5 \mathrm{H}), 4.12(\mathrm{q}, \mathrm{J}=7.1 \mathrm{~Hz}, 2 \mathrm{H}), 3.97(\mathrm{dd}, \mathrm{J}=10.1,5.4 \mathrm{~Hz}, 1 \mathrm{H}), 3.11(\mathrm{dd}, \mathrm{J}=16.7,10.1 \mathrm{~Hz}$, 1H), $2.61(\mathrm{dd}, \mathrm{J}=16.7,5.4 \mathrm{~Hz}, 1 \mathrm{H}), 1.39(\mathrm{~s}, 9 \mathrm{H}), 1.23(\mathrm{t}, \mathrm{J}=7.1 \mathrm{~Hz}, 3 \mathrm{H}) ;{ }^{13} \mathrm{C} \mathrm{NMR}(126$ $\left.\mathrm{MHz}_{\mathrm{CDCl}}\right) \delta 172.1,171.8,138.5,128.8,127.8,127.5,81.2,60.8,48.4,38.1,28.0$, 14.3; IR (film) 2979, 2932, 1724, 1496, 1454, 1409, 1392, 1367, 1349, 1329, 1294, 1248 , 1182, 1143, 1096, 1026, 1004, 992, 846, 783, 754, 728, $697 \mathrm{~cm}^{-1}$; LRMS (ESI): Mass calcd for $\mathrm{C}_{12} \mathrm{H}_{15} \mathrm{O}_{4}\left[\mathrm{M}-\mathrm{t}-\mathrm{BuO}+\mathrm{H}_{2} \mathrm{O}\right]^{+}:$223; found 223; Enantiomeric ratio was measured by chiral phase HPLC (Chiralcel IA, 1\% $i$-PrOH/Hexanes, $1.0 \mathrm{~mL} / \mathrm{min}, 210 \mathrm{~nm}$ ), Rt $($ major $)=8.26 \mathrm{~min}, \mathrm{Rt}($ minor $)=7.57 \mathrm{~min}$; er $=97: 3$.

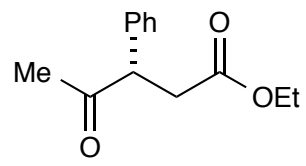

Ethyl (S)-4-oxo-3-phenylpentanoate (18): Prepared according to the general procedure using (E)-4-oxo-3-phenylpent-2-enal $(34.8 \mathrm{mg}, 0.200 \mathrm{mmol}$ ) and purified by flash chromatography using 8\% EtOAc/hexanes to afford $27 \mathrm{mg}(62 \%$ yield) of $\mathbf{1 8}$ as a colorless oil. Analytical data for 18: ${ }^{1} \mathrm{H}$ NMR $\left(500 \mathrm{MHz}, \mathrm{CDCl}_{3}\right) \delta 7.37-7.28(\mathrm{~m}, 3 \mathrm{H})$, $7.22-7.20(\mathrm{~m}, 2 \mathrm{H}), 4.18(\mathrm{dd}, \mathrm{J}=9.8,5.0 \mathrm{~Hz}, 1 \mathrm{H}), 4.10(\mathrm{tq}, \mathrm{J}=7.1,3.6 \mathrm{~Hz}, 2 \mathrm{H}), 3.20$ $(\mathrm{dd}, \mathrm{J}=17.0,9.8 \mathrm{~Hz}, 1 \mathrm{H}), 2.52(\mathrm{dd}, \mathrm{J}=17.0,5.0 \mathrm{~Hz}, 1 \mathrm{H}), 2.12(\mathrm{~s}, 3 \mathrm{H}), 1.22(\mathrm{t}, \mathrm{J}=7.1$ $\mathrm{Hz}, 3 \mathrm{H}) ;{ }^{13} \mathrm{C}$ NMR $\left(126 \mathrm{MHz}, \mathrm{CDCl}_{3}\right) \delta$ 207.0, 172.2, 137.5, 129.3, 128.4, 127.9, 60.8, 55.0, 37.2, 29.1, 14.3; IR (film) 3062, 3029, 2982, 2931, 1734, 1718, 1599, 1494, 1454, 
1409, 1394, 1374, 1353, 1322, 1291, 1240, 1189, 1157, 1114, 1096, 1028, 756, $701 \mathrm{~cm}^{-1}$; LRMS (ESI): Mass calcd for $\mathrm{C}_{13} \mathrm{H}_{17} \mathrm{O}_{3}[\mathrm{M}+\mathrm{H}]^{+}: 221$; found 221; Enantiomeric ratio was measured by chiral phase HPLC (Chiralcel ODH, 3\% $i$-PrOH/Hexanes, $1.0 \mathrm{~mL} / \mathrm{min}, 210$ $\mathrm{nm}), \mathrm{Rt}$ (major) $=10.7 \mathrm{~min}, \mathrm{Rt}($ minor $)=8.58 \mathrm{~min}$; er $=83: 17$.<smiles>CCOC(=O)C(CC(=O)OC)c1ccccc1</smiles>

1-ethyl 4-methyl (S)-2-phenylsuccinate (19): Prepared according to the general procedure using (E)-ethyl 4-oxo-2-phenylbut-2-enoate $(0.041 \mathrm{~g}, 0.200 \mathrm{mmol})$ and methanol $(0.081 \mathrm{~mL}, 2 \mathrm{mmol})$ and purified by flash chromatography using $8 \%$ Acetone/hexanes to afford $0.0469 \mathrm{~g}(99 \%)$ of $\mathbf{1 9}$ as a colorless oil. Analytical data for 19: ${ }^{1} \mathrm{H}$ NMR $\left(500 \mathrm{MHz}, \mathrm{CDCl}_{3}\right) \delta 7.32-7.27(\mathrm{~m}, 5 \mathrm{H}), 4.18-4.04(\mathrm{~m}, 3 \mathrm{H}), 3.67(\mathrm{~s}, 3 \mathrm{H})$, $3.20(\mathrm{dd}, \mathrm{J}=16.9,10.2 \mathrm{~Hz}, 1 \mathrm{H}), 2.66(\mathrm{dd}, \mathrm{J}=16.9,5.3 \mathrm{~Hz}, 1 \mathrm{H}), 1.20(\mathrm{t}, \mathrm{J}=7.1 \mathrm{~Hz}, 3 \mathrm{H})$; ${ }^{13} \mathrm{C} \mathrm{NMR}\left(126 \mathrm{MHz}, \mathrm{CDCl}_{3}\right) \delta 173.0,172.2,138.0,129.0,127.9,127.7,77.4,61.3,52.0$, 47.4, 37.8, 14.2; IR (film) 3031, 2982, 2953, 1727, 1496, 1454, 1436, 1409, 1365, 1329, 1294, 1250, 1226, 1197, 1154, 1113, 1095, 1072, 1021, 1004, 990, 856, 772, 731, 697 $\mathrm{cm}^{-1}$; LRMS (ESI): Mass calcd for $\mathrm{C}_{13} \mathrm{H}_{17} \mathrm{O}_{4}[\mathrm{M}+\mathrm{H}]^{+}$: 237; found 237; Enantiomeric ratio was measured by chiral phase HPLC (Chiralcel ODH, $1 \% i$-PrOH/Hexanes, $1.0 \mathrm{~mL} / \mathrm{min}$, $210 \mathrm{~nm})$, Rt $($ major $)=15.8 \mathrm{~min}, \mathrm{Rt}($ minor $)=28.3 \mathrm{~min}$; er $=91: 9$.

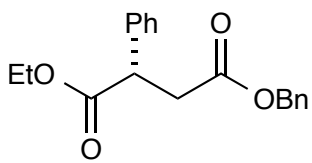

4-benzyl 1-ethyl (S)-2-phenylsuccinate (20): Prepared according to the general procedure using (E)-ethyl 4-oxo-2-phenylbut-2-enoate $(0.041 \mathrm{~g}, 0.200 \mathrm{mmol})$ and benzyl alcohol $(0.208 \mathrm{~mL}, 2 \mathrm{mmol})$ and purified by flash chromatography using $8 \%$ EtOAc/hexanes to afford $0.0506 \mathrm{~g}(81 \%)$ of $\mathbf{2 0}$ as a colorless oil. Analytical data for 20: ${ }^{1} \mathrm{H}$ NMR $\left(500 \mathrm{MHz}, \mathrm{CDCl}_{3}\right) \delta 7.35-7.27(\mathrm{~m}, 10 \mathrm{H}), 5.19-5.03(\mathrm{~m}, 2 \mathrm{H}), 4.19-4.02(\mathrm{~m}$, $3 \mathrm{H}), 3.25(\mathrm{dd}, \mathrm{J}=16.9,10.1 \mathrm{~Hz}, 1 \mathrm{H}), 2.73(\mathrm{dd}, \mathrm{J}=16.9,5.4 \mathrm{~Hz}, 1 \mathrm{H}), 1.17(\mathrm{t}, \mathrm{J}=7.1 \mathrm{~Hz}$, $3 \mathrm{H}) ;{ }^{13} \mathrm{C}$ NMR $\left(126 \mathrm{MHz}, \mathrm{CDCl}_{3}\right.$ ) $\delta$ 173.0, 171.5, 137.8, 135.8, 129.0, 128.7, 128.4, 128.3, 127.9, 127.7, 66.7, 61.3, 47.4, 38.0, 14.1; IR (film) 3089, 3065, 3033, 2982, 2939, 2905, 1730, 1602, 1497, 1455, 1408, 1384, 1371, 1329, 1294, 1254, 1228, 1156, 1095, 1021, 968, 909, 857, 731, 697, $648 \mathrm{~cm}^{-1}$; LRMS (ESI): Mass calcd for $\mathrm{C}_{19} \mathrm{H}_{21} \mathrm{O}_{4}[\mathrm{M}+\mathrm{H}]^{+}$: 313; found 313; Enantiomeric ratio was measured by chiral phase HPLC (Chiralcel Whelk-O, 5\% $i$-PrOH/Hexanes, $1.0 \mathrm{~mL} / \mathrm{min}, 210 \mathrm{~nm}$ ), Rt (major) = $23.2 \mathrm{~min}$, Rt (minor) $=20.7 \mathrm{~min}$; er $=93: 7$.

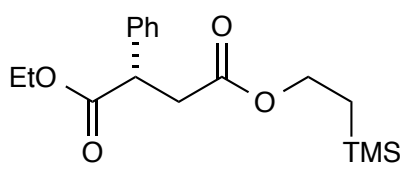

1-ethyl 4-(2-(trimethylsilyl)ethyl) (S)-2-phenylsuccinate (21): Prepared according to the general procedure using (E)-ethyl 4-oxo-2-phenylbut-2-enoate $(0.041 \mathrm{~g}, 0.200 \mathrm{mmol})$ and 2-(trimethylsilyl)ethanol $(0.287 \mathrm{~mL}, 2 \mathrm{mmol})$ and purified by flash chromatography using $8 \%$ Acetone/hexanes to afford $0.0398 \mathrm{~g}(62 \%)$ of $\mathbf{2 1}$ as a colorless oil. Analytical 
data for 21: ${ }^{1} \mathrm{H}$ NMR $\left(500 \mathrm{MHz}, \mathrm{CDCl}_{3}\right) \delta 7.33-7.28(\mathrm{~m}, 5 \mathrm{H}), 4.20-4.11(\mathrm{~m}, 4 \mathrm{H}), 4.06$ $(\mathrm{dd}, \mathrm{J}=10.2,5.2 \mathrm{~Hz}, 1 \mathrm{H}), 3.17(\mathrm{dd}, \mathrm{J}=16.9,10.2 \mathrm{~Hz}, 1 \mathrm{H}), 2.63(\mathrm{dd}, \mathrm{J}=16.9,5.2 \mathrm{~Hz}$, $1 \mathrm{H}), 1.20(\mathrm{t}, \mathrm{J}=7.1 \mathrm{~Hz}, 3 \mathrm{H}), 0.95(\mathrm{dd}, \mathrm{J}=9.1,8.0 \mathrm{~Hz}, 2 \mathrm{H}), 0.02(\mathrm{~s}, 9 \mathrm{H}) ;{ }^{13} \mathrm{C}$ NMR $(126$ $\left.\mathrm{MHz} \mathrm{CDCl}_{3}\right) \delta 173.1,171.8,138.1,128.9,127.9,127.7,63.1,61.2,47.4,38.2,17.4$, 14.2, -1.4; IR (film) 2978, 2954, 2899, 1728, 1454, 1385, 1370, 1343, 1327, 1293, 1248, 1225, 1154, 1113, 1096, 1065, 1038, 1021, 970, 937, 856, 834, 764, 729, 696, $663 \mathrm{~cm}^{-1}$; LRMS (ESI): Mass calcd for $\mathrm{C}_{34} \mathrm{H}_{52} \mathrm{NaO}_{8} \mathrm{Si}_{2}$ [2M+Na] ${ }^{+}$: 667; found 667; Enantiomeric ratio was measured by chiral phase HPLC (Chiralcel ADH, 1\% $i$-PrOH/Hexanes, 1.0 $\mathrm{mL} / \mathrm{min}, 210 \mathrm{~nm})$, Rt (major) $=11.4 \mathrm{~min}$, Rt $($ minor $)=8.48 \mathrm{~min}$; er $=91: 9$.

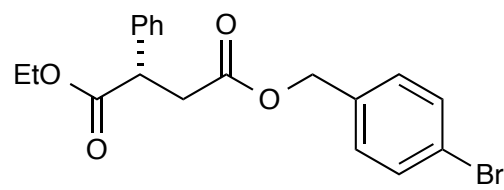

4-(4-bromobenzyl) 1-ethyl (S)-2-phenylsuccinate (22): Prepared according to the general procedure using (E)-ethyl 4-oxo-2-phenylbut-2-enoate $(0.041 \mathrm{~g}, 0.200 \mathrm{mmol})$ and (4-bromophenyl)methanol $(0.374 \mathrm{~g}, 2 \mathrm{mmol})$ and purified by flash chromatography using $10 \% \mathrm{EtOAc/hexanes} \mathrm{to} \mathrm{afford} 0.0554 \mathrm{~g}(71 \%)$ of 22 as a white solid. Analytical data for 22: ${ }^{1} \mathrm{H}$ NMR $\left(500 \mathrm{MHz}, \mathrm{CDCl}_{3}\right) \delta 7.49-7.44(\mathrm{~m}, 2 \mathrm{H}), 7.32-7.27(\mathrm{~m}, 5 \mathrm{H}), 7.16(\mathrm{~d}, \mathrm{~J}=$ $8.4 \mathrm{~Hz}, 2 \mathrm{H}), 5.10-4.99(\mathrm{~m}, 2 \mathrm{H}), 4.15-4.04(\mathrm{~m}, 3 \mathrm{H}), 3.24(\mathrm{dd}, \mathrm{J}=16.9,10.0 \mathrm{~Hz}, 1 \mathrm{H})$, $2.72(\mathrm{dd}, \mathrm{J}=16.9,5.5 \mathrm{~Hz}, 1 \mathrm{H}), 1.17(\mathrm{t}, \mathrm{J}=7.1 \mathrm{~Hz}, 3 \mathrm{H}) ;{ }^{13} \mathrm{C} \mathrm{NMR}\left(126 \mathrm{MHz}, \mathrm{CDCl}_{3}\right) \delta$ $172.9,171.4,137.7,134.8,131.8,130.0,129.0,127.9,127.8,122.4,65.8,61.4,47.4$, 38.0, 14.1; IR (film) 3088, 3063, 3031, 2980, 2934, 2849, 1730, 1595, 1489, 1454, 1406, 1370, 1329, 1293, 1252, 1228, 1154, 1095, 1070, 1013, 855, 832, $800 \mathrm{~cm}^{-1}$; LRMS (ESI): Mass calcd for $\mathrm{C}_{19} \mathrm{H}_{20} \mathrm{BrO}_{4}[\mathrm{M}+\mathrm{H}]^{+}: 393$; found 393; Enantiomeric ratio was measured by chiral phase HPLC (Chiralcel Whelk-O, 5\% $i$-PrOH/Hexanes, $1.0 \mathrm{~mL} / \mathrm{min}, 210 \mathrm{~nm}$ ), Rt $($ major $)=26.6 \mathrm{~min}, \mathrm{Rt}($ minor $)=22.9 \mathrm{~min}$; er $=93: 7$. After recrystallization from slow evaporation of hexanes, $0.0497 \mathrm{~g}(64 \%$ yield $)$ of $\mathbf{2 2}$, er $=99: 1$.

\section{General Procedures for the Synthetic Transformations}<smiles>CCOC(=O)C[C@@H](C(=O)O)c1ccccc1</smiles>

(S)-4-ethoxy-4-oxo-2-phenylbutanoic acid (23): In a $20 \mathrm{~mL}$ vial with a stir bar was added 1-benzyl 4-ethyl 2-phenylsuccinate $(0.544 \mathrm{mmol}), 10 \%$ palladium/carbon $(0.054$ mmol), and EtOAc (3.6 mL). The system was purged with $\mathrm{H}_{2}$ via a hydrogen balloon, and the reaction mixture was stirred overnight under an $\mathrm{H}_{2}$ atmosphere. The reaction mixture was filtered through a Celite plug, eluting with EtOAc, and concentrated to afford $120 \mathrm{mg}$ (99\% yield) of $\mathbf{2 3}$ as a white solid. Analytical data for 23: ${ }^{1} \mathrm{H}$ NMR (500

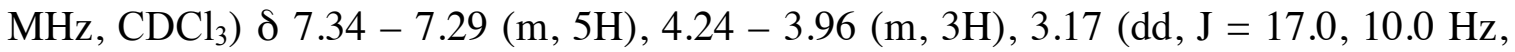
$1 \mathrm{H}), 2.68(\mathrm{dd}, \mathrm{J}=17.0,5.3 \mathrm{~Hz}, 1 \mathrm{H}), 1.21(\mathrm{t}, \mathrm{J}=7.2 \mathrm{~Hz}, 3 \mathrm{H}) ;{ }^{13} \mathrm{C}$ NMR $(126 \mathrm{MHz}$, $\left.\mathrm{CDCl}_{3}\right) \delta 177.0,171.5,137.1,129.1,128.1,128.0,61.0,46.9,37.6,14.2$; IR (film) 3255, 3032, 2982, 2917, 2849, 1734, 1709, 1602, 1585, 1540, 1497, 1455, 1418, 1395,1374, 1279, 1252, 1164, 1095, 1024, 856, 762, 726, 698, $656 \mathrm{~cm}^{-1}$; LRMS (ESI): Mass calcd for $\mathrm{C}_{12} \mathrm{H}_{5} \mathrm{O}_{4}[\mathrm{M}+\mathrm{H}]^{+}:$223; found 223 . 
<smiles>CCOC(=O)[C@H](CC(=O)O)c1ccccc1</smiles>

(S)-4-ethoxy-4-oxo-3-phenylbutanoic acid (24): In a $20 \mathrm{~mL}$ vial with a stir bar was added 4-benzyl 1-ethyl 2-phenylsuccinate $(1.201 \mathrm{mmol}), 10 \%$ palladium/carbon $(0.120$ mmol), and EtOAc $(8.0 \mathrm{~mL})$. The system was purged with $\mathrm{H}_{2}$ via a hydrogen balloon, and the reaction mixture was stirred overnight under an $\mathrm{H}_{2}$ atmosphere. The reaction mixture was filtered through a Celite plug, eluting with EtOAc, and concentrated to afford $265 \mathrm{mg}$ (99\% yield) of $\mathbf{2 4}$ as a white solid. Analytical data for $\mathbf{2 4}:{ }^{1} \mathrm{H}$ NMR (500 $\left.\mathrm{MHz}, \mathrm{CDCl}_{3}\right) \delta 7.33-7.28(\mathrm{~m}, 5 \mathrm{H}), 4.14(\mathrm{ddd}, \mathrm{J}=38.9,10.8,7.1 \mathrm{~Hz}, 2 \mathrm{H}), 4.04(\mathrm{dd}, \mathrm{J}=$ $10.2,5.0 \mathrm{~Hz}, 1 \mathrm{H}), 3.26(\mathrm{dd}, \mathrm{J}=17.3,10.2 \mathrm{~Hz}, 1 \mathrm{H}), 2.71(\mathrm{dd}, \mathrm{J}=17.3,5.1 \mathrm{~Hz}, 1 \mathrm{H}), 1.19$ $(\mathrm{t}, \mathrm{J}=7.1 \mathrm{~Hz}, 3 \mathrm{H})$.; ${ }^{13} \mathrm{C} \mathrm{NMR}\left(126 \mathrm{MHz}, \mathrm{CDCl}_{3}\right) \delta 172.8,137.5,128.9,127.7,61.3,47.0$, 37.4, 14.0; IR (film) 3255, 3032, 2982, 2936, 1731, 1711, 1618, 1585, 1497, 1454, 1402, 1371, 1295, 1263, 1230, 1174, 1020, $699 \mathrm{~cm}^{-1}$; LRMS (ESI): Mass calcd for $\mathrm{C}_{12} \mathrm{H}_{5} \mathrm{O}_{4}$ $[\mathrm{M}+\mathrm{H}]^{+}:$223; found 223 .

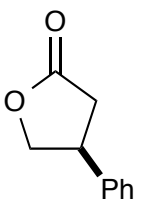

(R)-ethyl 2-phenylcyclopent-2-enecarboxylate (25) ${ }^{\mathbf{5}}$ : In a oven dried $20 \mathrm{~mL}$ vial with a stir bar was added 4-ethoxy-4-oxo-2-phenylbutanoic acid (0.225 mmol), and THF (1.5 $\mathrm{mL}$ ). The solution was cooled to $0{ }^{\circ} \mathrm{C}$ and (tetrahydro-1H-furan-1-ium-1yl)trihydroborate $(337 \mu \mathrm{L}, 0.337 \mathrm{mmol})$ was added dropwise by a syringe. Stirred at $0^{\circ} \mathrm{C}$ for 15 minutes and then warmed to $23{ }^{\circ} \mathrm{C}$ overnight. Cooled to $-10{ }^{\circ} \mathrm{C}$ and trifluoroborate dietherate $(70 \mu \mathrm{L}, 0.567 \mathrm{mmol})$ was added by syringe. Stirred at 0 to $23{ }^{\circ} \mathrm{C}$ overnight. Quenched by the addition of brine and extracted with EtOAc, dried over $\mathrm{MgSO}_{4}$, filtered and concentrated. Purified by flash column chromatography 30\% EtOAc/hexanes to afford $32 \mathrm{mg}$ (86\% yield) of $\mathbf{2 5}$ the product lactone as an oil. Analytical data for 25: ${ }^{1} \mathrm{H}$ NMR $\left(500 \mathrm{MHz} \mathrm{CDCl}_{3}\right) \delta 7.38(\mathrm{t}, \mathrm{J}=7.4 \mathrm{~Hz}, 2 \mathrm{H}), 7.31(\mathrm{t}, \mathrm{J}=7.4 \mathrm{~Hz}, 1 \mathrm{H}), 7.24(\mathrm{~d}, \mathrm{~J}=$ $7.2 \mathrm{~Hz}, 2 \mathrm{H}), 4.75-4.62(\mathrm{~m}, 1 \mathrm{H}), 4.33-4.25(\mathrm{~m}, 1 \mathrm{H}), 3.80(\mathrm{p}, \mathrm{J}=8.4 \mathrm{~Hz}, 1 \mathrm{H}), 2.94$ (dd, $\mathrm{J}=17.5,8.7 \mathrm{~Hz}, 1 \mathrm{H}), 2.69(\mathrm{dd}, \mathrm{J}=17.5,9.2 \mathrm{~Hz}, 1 \mathrm{H}) ;{ }^{13} \mathrm{C} \mathrm{NMR}\left(126 \mathrm{MHz}, \mathrm{CDCl}_{3}\right) \delta$ 176.6, 139.5, 129.3, 127.9, 126.8, 74.2, 41.3, 35.9; IR (film) 3032, 2962, 2916, 2849, 2349, 1781, 1603, 1542, 1496, 1473, 1455, 1419, 1376, 1350, 1285, 1225, 1207, 1167 , 1041, 1020, 1000, 991, 947, 898, 854, 850, 818, 762, 751, 700, 671, 664, 656, $641 \mathrm{~cm}^{-1}$; LRMS (ESI): Mass calcd for $\mathrm{C}_{10} \mathrm{H}_{11} \mathrm{O}_{2}[\mathrm{M}+\mathrm{H}]^{+}:$: 163; found 163; Enantiomeric ratio was measured by chiral phase HPLC (Chiralcel IA, 1\% $i$-PrOH/Hexanes, $1.0 \mathrm{~mL} / \mathrm{min}, 210$ $\mathrm{nm}), \operatorname{Rt}($ major $)=36.2 \mathrm{~min}$, Rt (minor) $=42.1 \mathrm{~min}$; er $=94: 6$.

\footnotetext{
${ }^{5}$ L. Zhou, X. Liu, J. Ji, Y. Zhang, X. Hu, L. Lin, X. Feng, J. Am. Chem. Soc. 2012, 134, 17023-17026.
} 


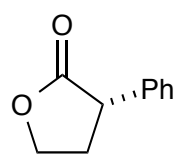

(S)-3-phenyldihydrofuran-2(3H)-one (26) ${ }^{\mathbf{6}}$ : Prepared analogously to 25 , using 24 $(0.225 \mathrm{mmol})$ to afford $27 \mathrm{mg}$ (74\% yield) of $\mathbf{2 6}$ as a clear liquid. Analytical data for $\mathbf{2 6}$ : ${ }^{1} \mathrm{H}$ 1H NMR (500 MHz, $\mathrm{CDCl}_{3}$ ) $\delta 7.38(\mathrm{t}, \mathrm{J}=7.3 \mathrm{~Hz}, 2 \mathrm{H}), 7.31$ (ddd, J = 8.6, 7.2, $2.5 \mathrm{~Hz}$, $3 \mathrm{H}), 4.49(\mathrm{td}, \mathrm{J}=8.7,3.3 \mathrm{~Hz}, 1 \mathrm{H}), 4.36(\mathrm{td}, \mathrm{J}=9.2,6.7 \mathrm{~Hz}, 1 \mathrm{H}), 3.87-3.78(\mathrm{~m}, 1 \mathrm{H})$, $2.78-2.67(\mathrm{~m}, 1 \mathrm{H}), 2.51-2.40(\mathrm{~m}, 1 \mathrm{H}) ;{ }^{13} \mathrm{C} \mathrm{NMR}\left(126 \mathrm{MHz}, \mathrm{CDCl}_{3}\right) \delta$ 177.5, 136.7, 129.1 , 128.0, 127.8, 66.6, 45.6, 31.8; IR (film) 3063, 3030, 2988, 2915, 1766, 1732,1682, 1651, 1602, 1497, 1454, 1372, 1266, 1216, 1148, 1096, 1069, 1022, 995, 952, 918, 805, 751, 697, 667, 662, $626 \mathrm{~cm}^{-1}$; LRMS (ESI): Mass calcd for $\mathrm{C}_{10} \mathrm{H}_{11} \mathrm{O}_{2}[\mathrm{M}+\mathrm{H}]^{+}: 163$; found 163; Enantiomeric ratio was measured by chiral phase HPLC (Chiralcel IA, 3\% $i$ $\mathrm{PrOH} / \mathrm{Hexanes}, 1.0 \mathrm{~mL} / \mathrm{min}, 210 \mathrm{~nm})$, Rt (major) $=28.2 \mathrm{~min}$, Rt $($ minor $)=25.5 \mathrm{~min}$; er $=$ 91:9.<smiles>CCOC(=O)CC(NC(=O)c1ccccc1)c1ccccc1</smiles>

Ethyl (S)-3-((tert-butoxycarbonyl)amino)-3-phenylpropanoate $(27)^{7}$ : In a oven dried 2 dram vial with a stir bar was added 4-ethoxy-4-oxo-2-phenylbutanoic acid $(0.225$ $\mathrm{mmol}), t$ - BuOH $(0.5 \mathrm{~mL})$, triethylamine $(33 \mu \mathrm{L}, 0.237 \mathrm{mmol})$, and diphenyl phosphorazidate $(51 \mu \mathrm{L}, 0.237 \mathrm{mmol})$, heated at $85^{\circ} \mathrm{C}$ for $48 \mathrm{~h}$. Then, the mixture was cooled to $23{ }^{\circ} \mathrm{C}$, diluted with EtOAc $(5 \mathrm{~mL})$, washed with saturated $\mathrm{NaHCO}_{3}(5 \mathrm{~mL})$, $\mathrm{H}_{2} \mathrm{O}(5 \mathrm{~mL})$, and brine. The organic layer dried over $\mathrm{MgSO}_{4}$, filtered and concentrated. Purified by flash column chromatography $10 \%$ EtOAc/hexanes to afford $47 \mathrm{mg}$ (71\% yield) of 27 as a colorless oil. Analytical data for 27 : ${ }^{1} \mathrm{H} \mathrm{NMR}\left(500 \mathrm{MHz}, \mathrm{CDCl}_{3}\right) \delta 7.31$ $(\mathrm{dt}, \mathrm{J}=14.1,7.3 \mathrm{~Hz}, 5 \mathrm{H}), 5.47(\mathrm{~s}, 1 \mathrm{H}), 5.11(\mathrm{~s}, 1 \mathrm{H}), 4.06(\mathrm{q}, \mathrm{J}=7.1 \mathrm{~Hz}, 2 \mathrm{H}), 2.93-2.73$ $(\mathrm{m}, 2 \mathrm{H}), 1.42(\mathrm{~s}, 9 \mathrm{H}), 1.16(\mathrm{t}, \mathrm{J}=7.1 \mathrm{~Hz}, 3 \mathrm{H}) ;{ }^{13} \mathrm{C} \mathrm{NMR}\left(126 \mathrm{MHz}, \mathrm{CDCl}_{3}\right) \delta 171.0$, 155.0, 141.1, 128.6, 127.5, 126.2, 79.7, 60.7, 51.2, 41.0, 28.4, 14.1; IR (film) 3360, 3064, 3032, 3979, 3931, 1715, 1496, 1455, 1391, 1366, 1289, 1246, 1166, 1096, 1080, 1044, 1023, 963, 872, 757, 699, 667, $656 \mathrm{~cm}^{-1}$; LRMS (ESI): Mass calcd for $\mathrm{C}_{16} \mathrm{H}_{24} \mathrm{NO}_{4}$ $[\mathrm{M}+\mathrm{H}]^{+}:$294; found 294; Enantiomeric ratio was measured by chiral phase HPLC (Chiralcel ADH, 5\% i-PrOH/Hexanes, $1.0 \mathrm{~mL} / \mathrm{min}, 254 \mathrm{~nm}$ ), Rt (major) = $18.3 \mathrm{~min}$, Rt (minor) $=15.4 \mathrm{~min}$; er $=94: 6$.<smiles>CCOC(=O)[C@H](CNC(C)(C)C)c1ccccc1</smiles>

Ethyl (R)-3-((tert-butoxycarbonyl)amino)-2-phenylpropanoate (28): Prepared analogously to $\mathbf{2 7}$, using $\mathbf{2 4}(65 \mathrm{mg}, 0.292 \mathrm{mmol})$ to afford $61 \mathrm{mg}$ (71\% yield) of $\mathbf{2 8}$ as a colorless oil. Analytical data for 28: ${ }^{1} \mathrm{H}$ NMR $\left(500 \mathrm{MHz}, \mathrm{CDCl}_{3}\right) \delta 7.35-7.27(\mathrm{~m}, 5 \mathrm{H})$, $4.86(\mathrm{~s}, 1 \mathrm{H}), 4.27-4.06(\mathrm{~m}, 2 \mathrm{H}), 3.92-3.81(\mathrm{~m}, 1 \mathrm{H}), 3.64-3.55(\mathrm{~m}, 1 \mathrm{H}), 3.55-3.44$

\footnotetext{
${ }^{6}$ Z. Huang, Z. Chen, L. H. Lim, G. C. P. Quang, H. Hirao, J. Zhou, Angew. Chem. Int. Ed. 2013, 52, 58075812.

${ }^{7}$ G. A. Molander, P. J. Stengel, Tetrahedron 1997, 53, 8887-8912.
} 
$(\mathrm{m}, 1 \mathrm{H}), 1.42(\mathrm{~s}, 9 \mathrm{H}), 1.21(\mathrm{t}, \mathrm{J}=7.1 \mathrm{~Hz}, 3 \mathrm{H}) ;{ }^{13} \mathrm{C} \mathrm{NMR}\left(126 \mathrm{MHz}, \mathrm{CDCl}_{3}\right) \delta 173.1$, 155.9, 136.7, 129.0, 128.1 , 127.7, 79.6, 61.2, 51.8, 43.6, 28.5, 14.2; IR (film) 3380, 3064, 3031, 2978, 2934, 1718, 1507, 1455, 1392, 1366, 1279, 1250, 1197, 1171, $699 \mathrm{~cm}^{-1}$; LRMS (ESI): Mass calcd for $\mathrm{C}_{16} \mathrm{H}_{24} \mathrm{NO}_{4}[\mathrm{M}+\mathrm{H}]^{+}$: 294; found 294; Enantiomeric ratio was measured by chiral phase HPLC (Chiralcel ODH, $0.5 \% i$-PrOH/Hexanes, $0.5 \mathrm{~mL} / \mathrm{min}$, $210 \mathrm{~nm})$, Rt (major) $=46.0 \mathrm{~min}$, Rt $($ minor $)=39.7 \mathrm{~min}$; er $=93: 7$. 


\section{Selected NMR Spectra}
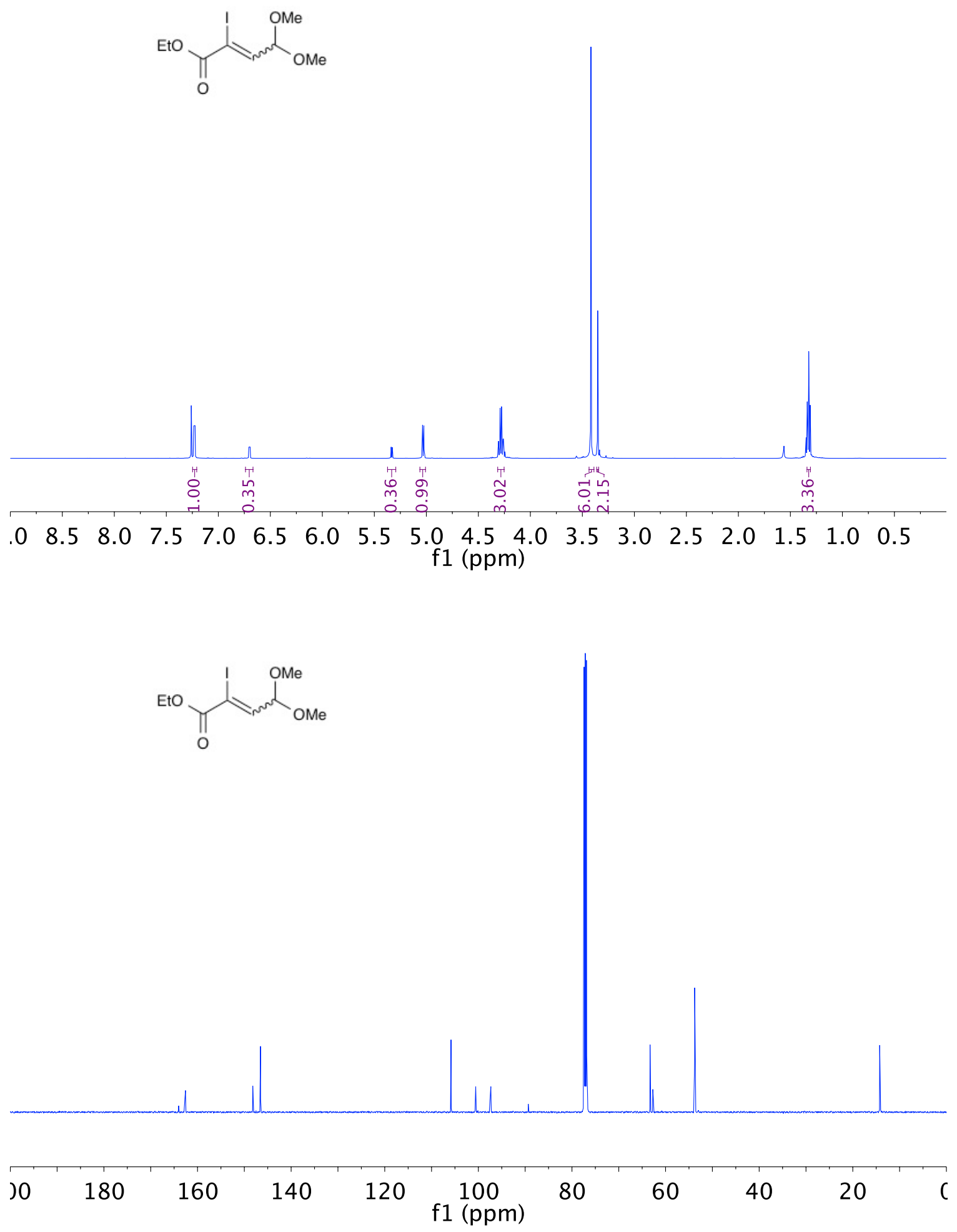

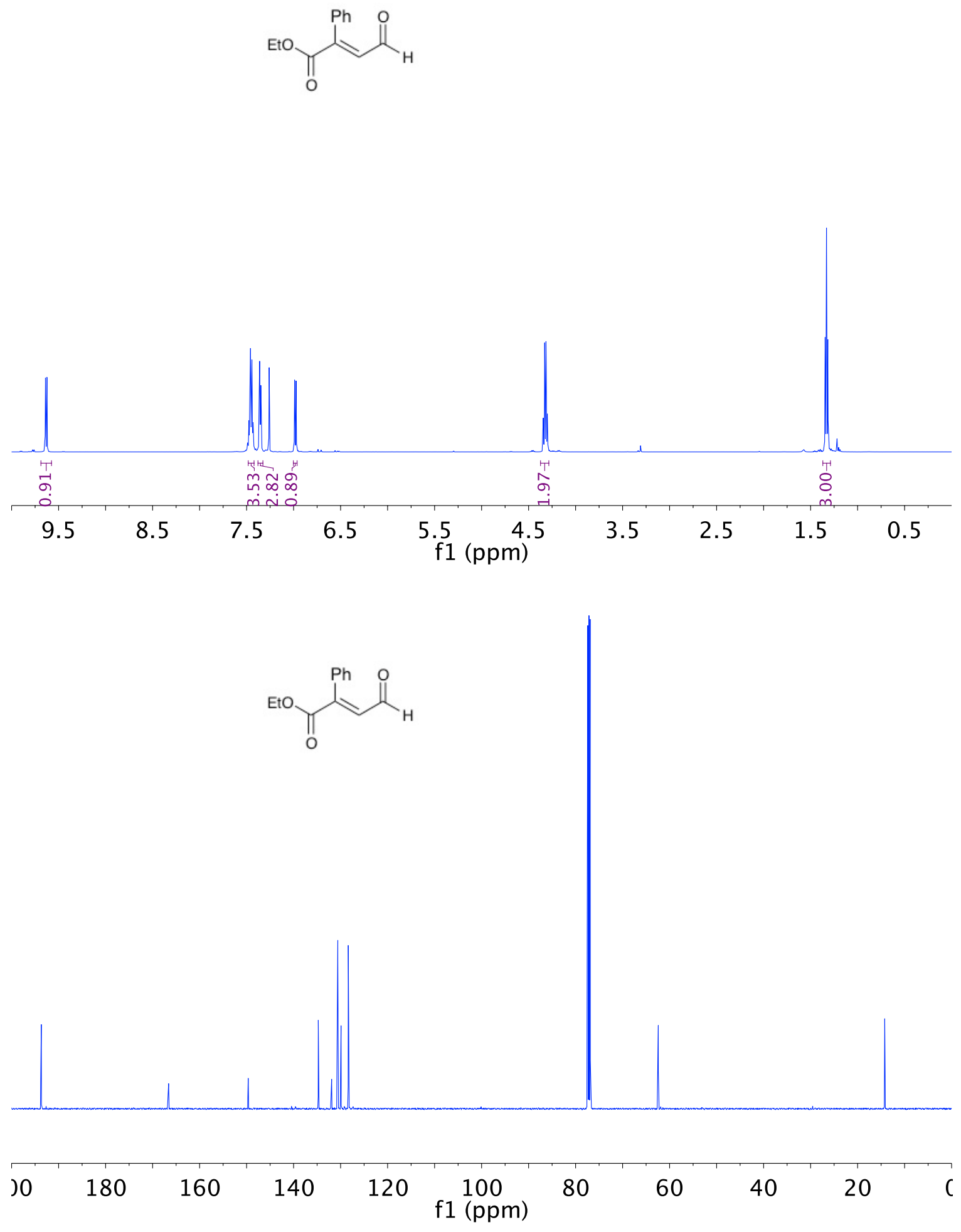

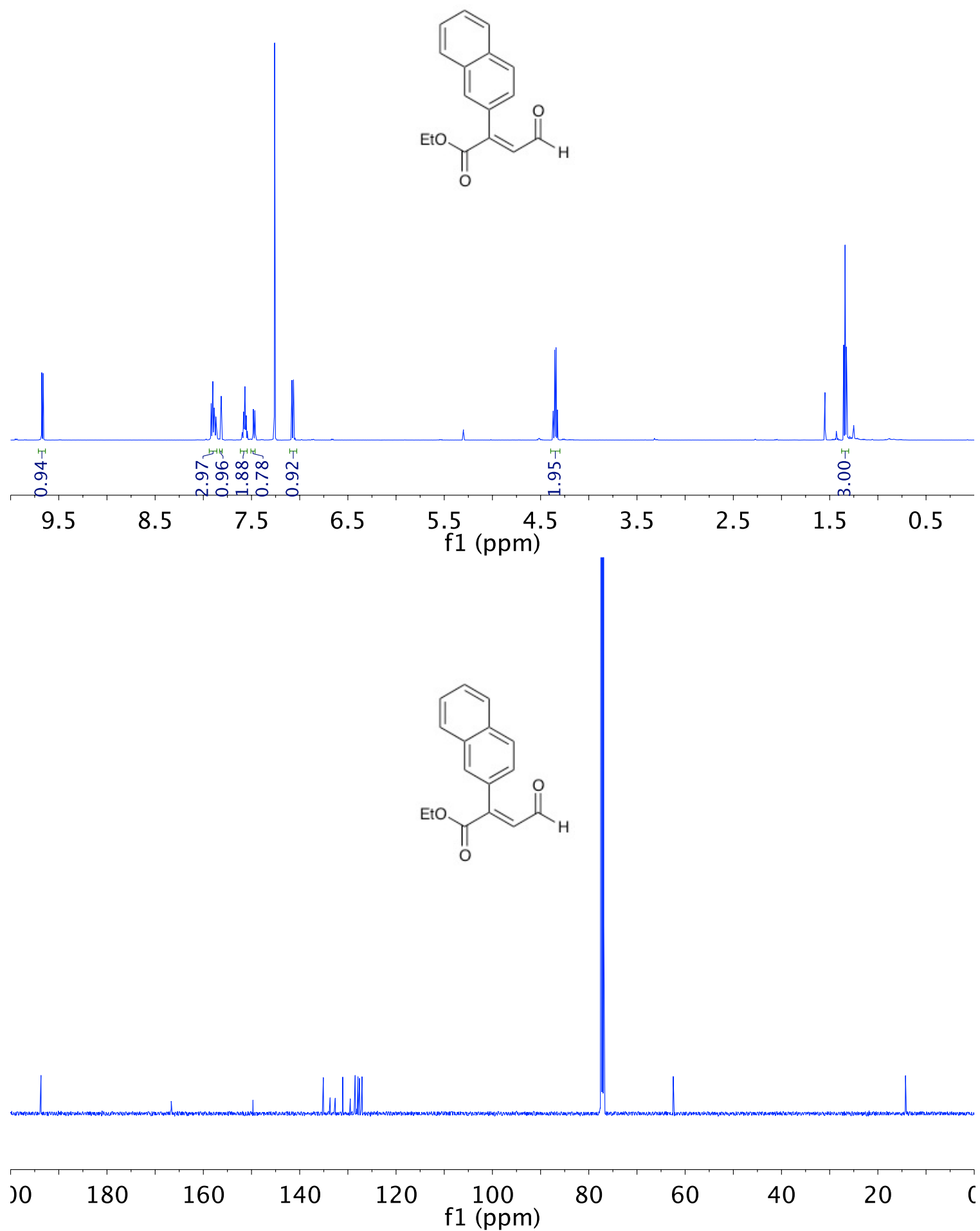

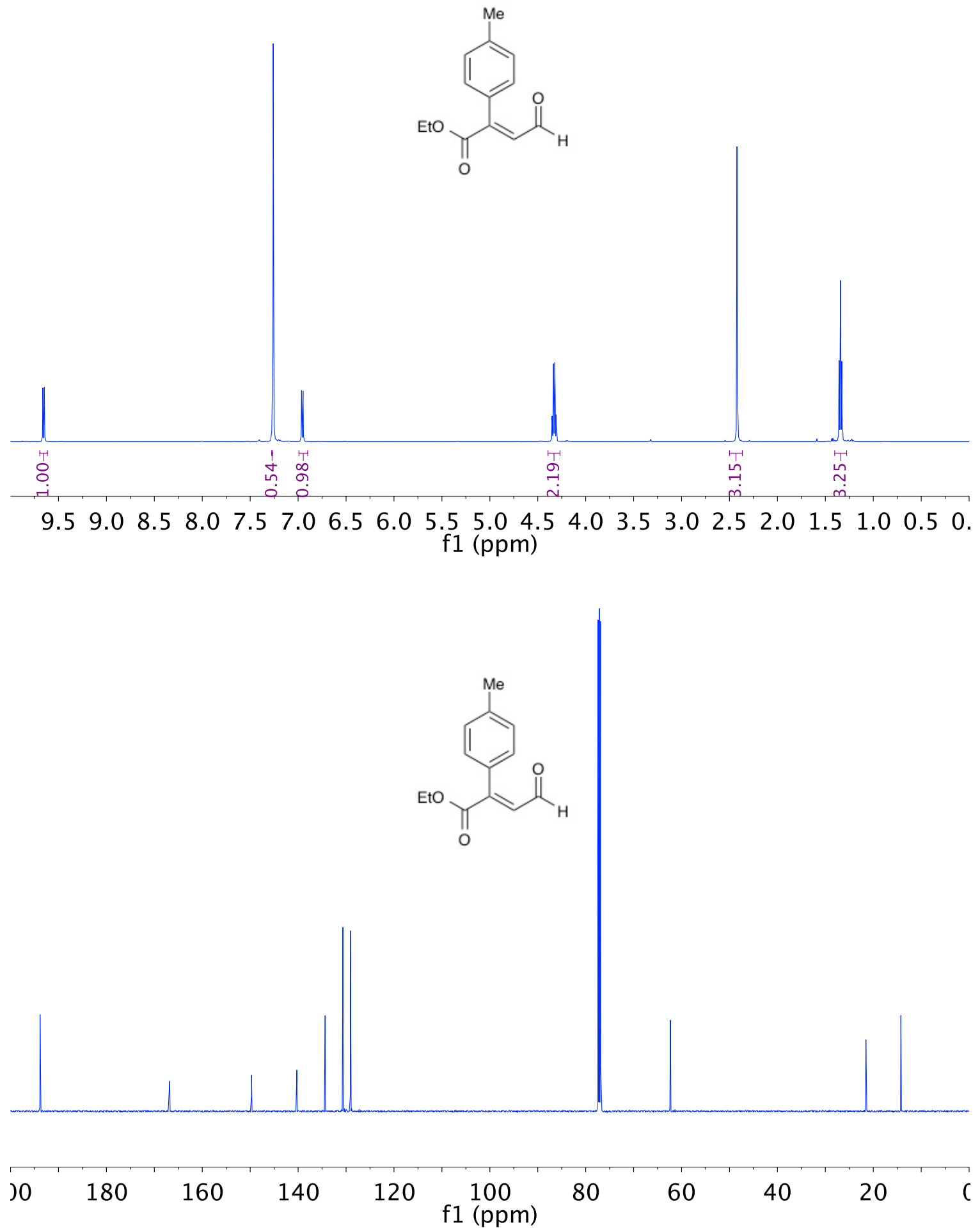

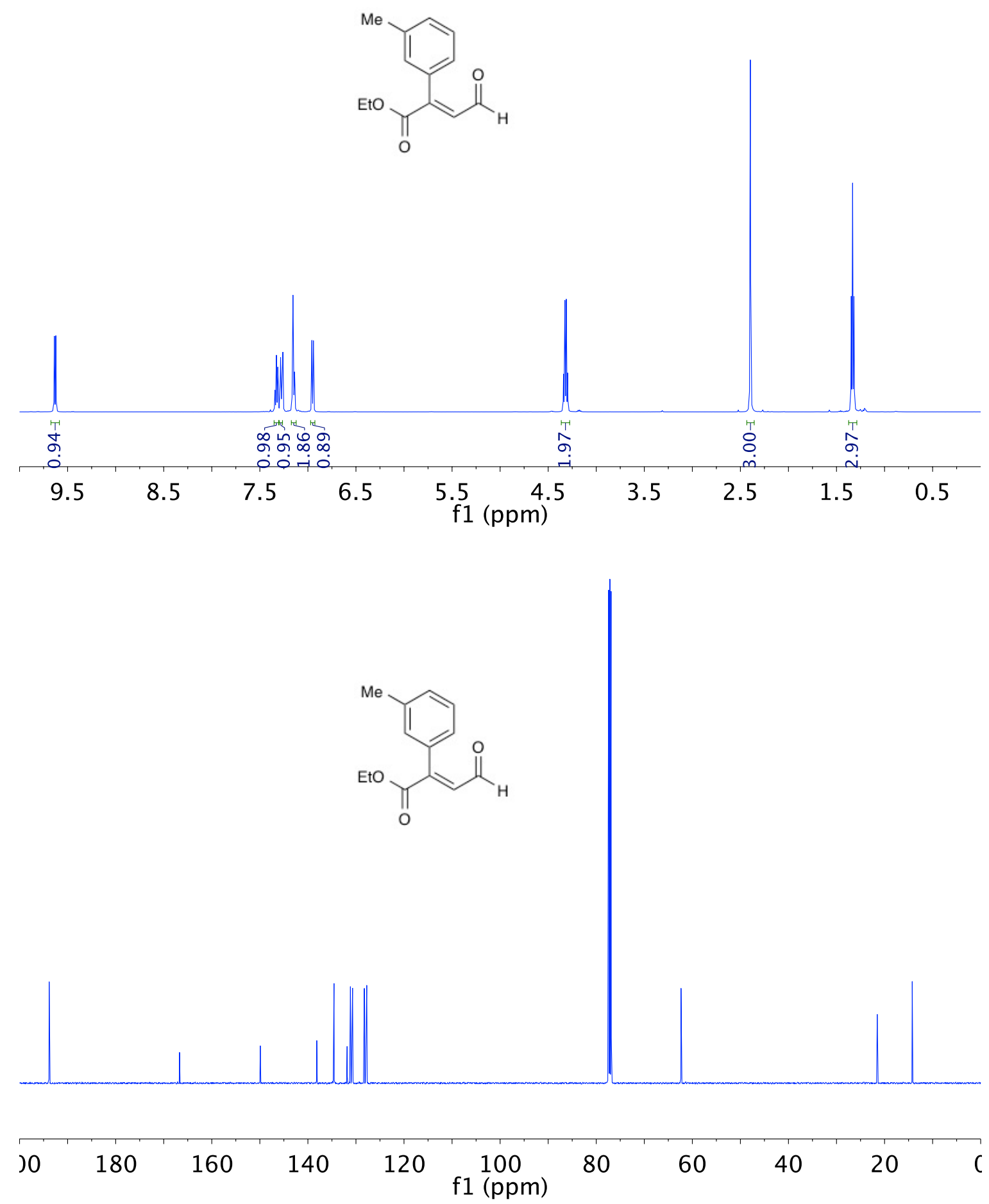

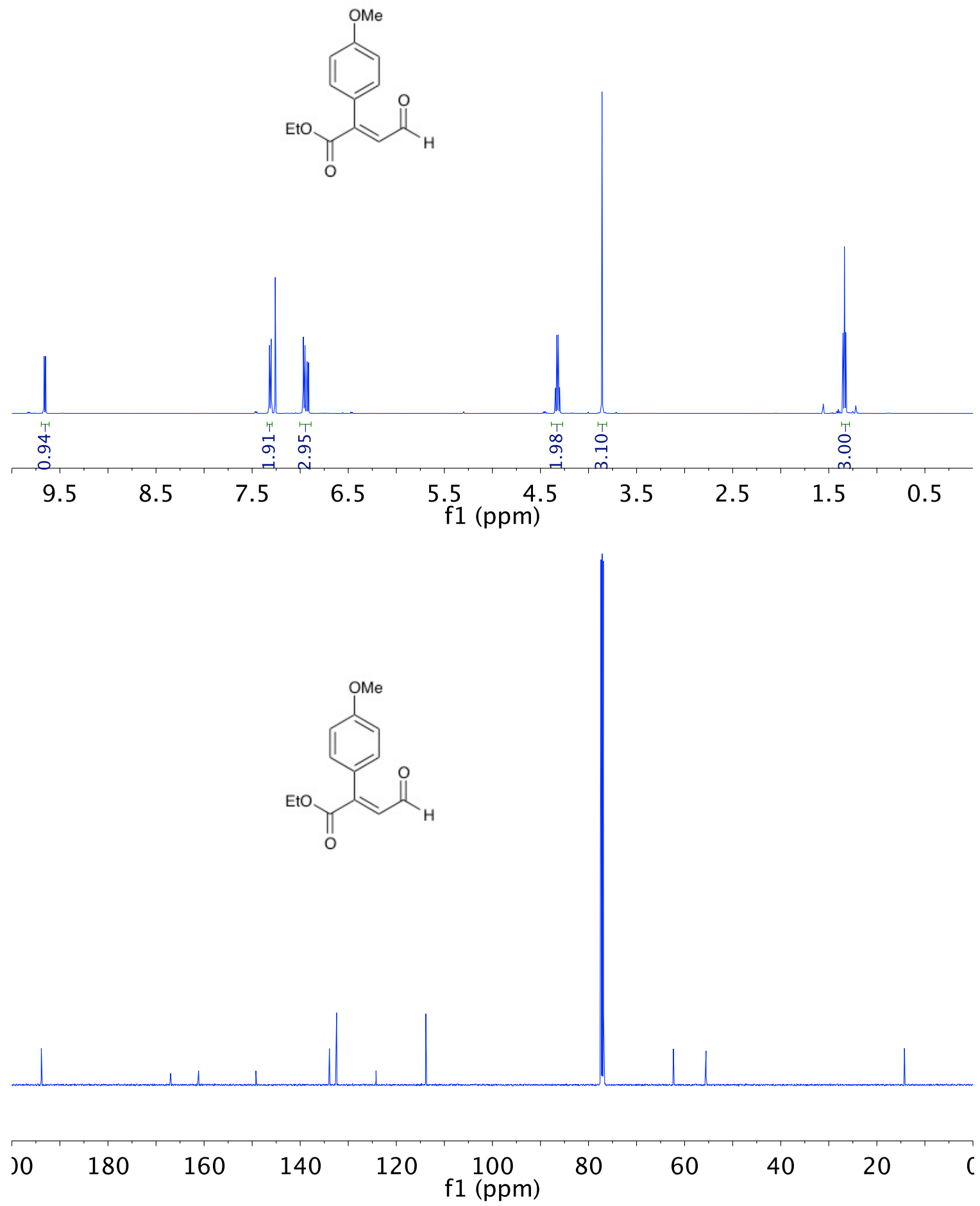

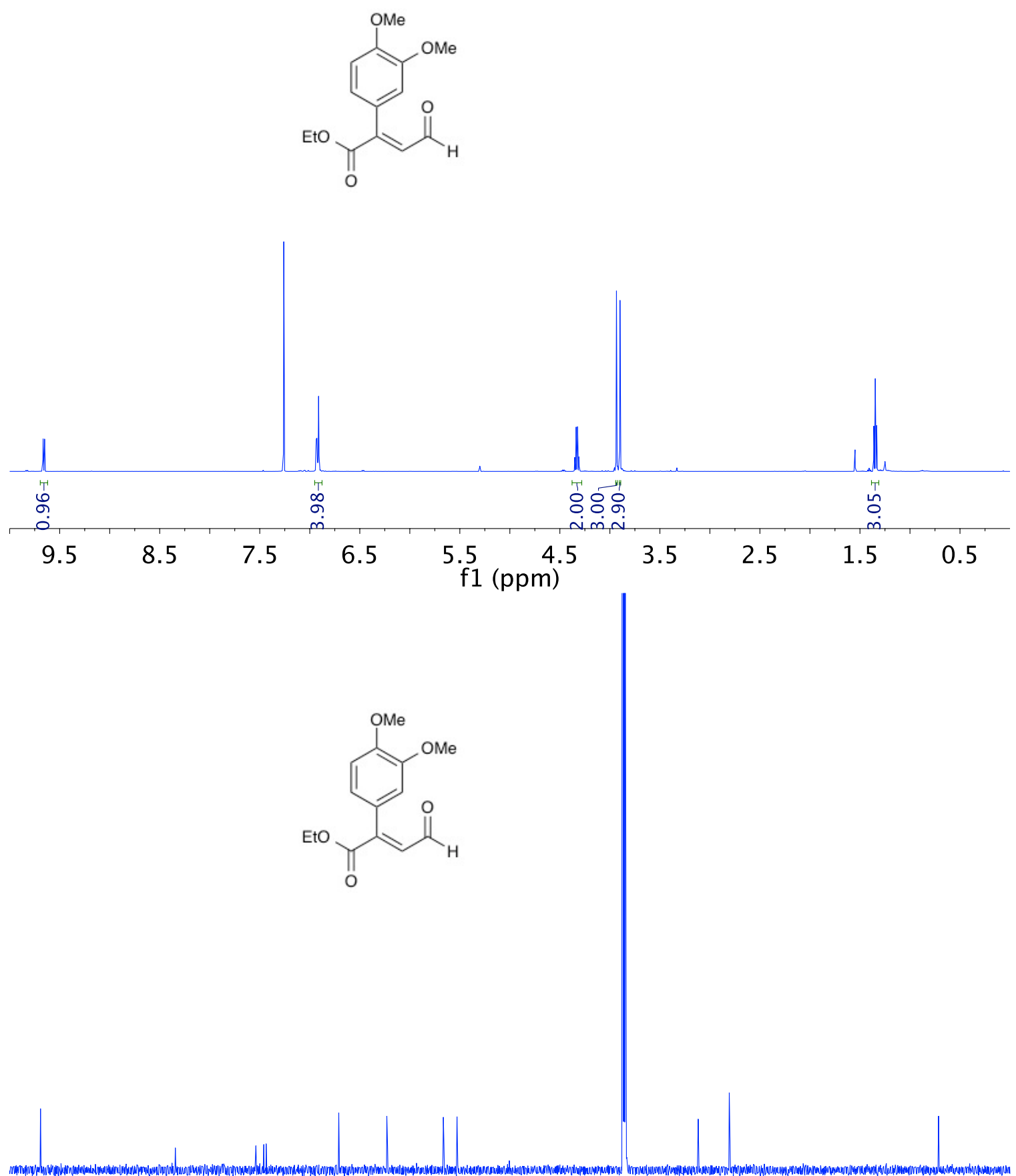

$50180 \quad 160 \quad 140 \quad 120 \begin{gathered}100 \\ \mathrm{f} 1 \mathrm{(ppm})\end{gathered}$



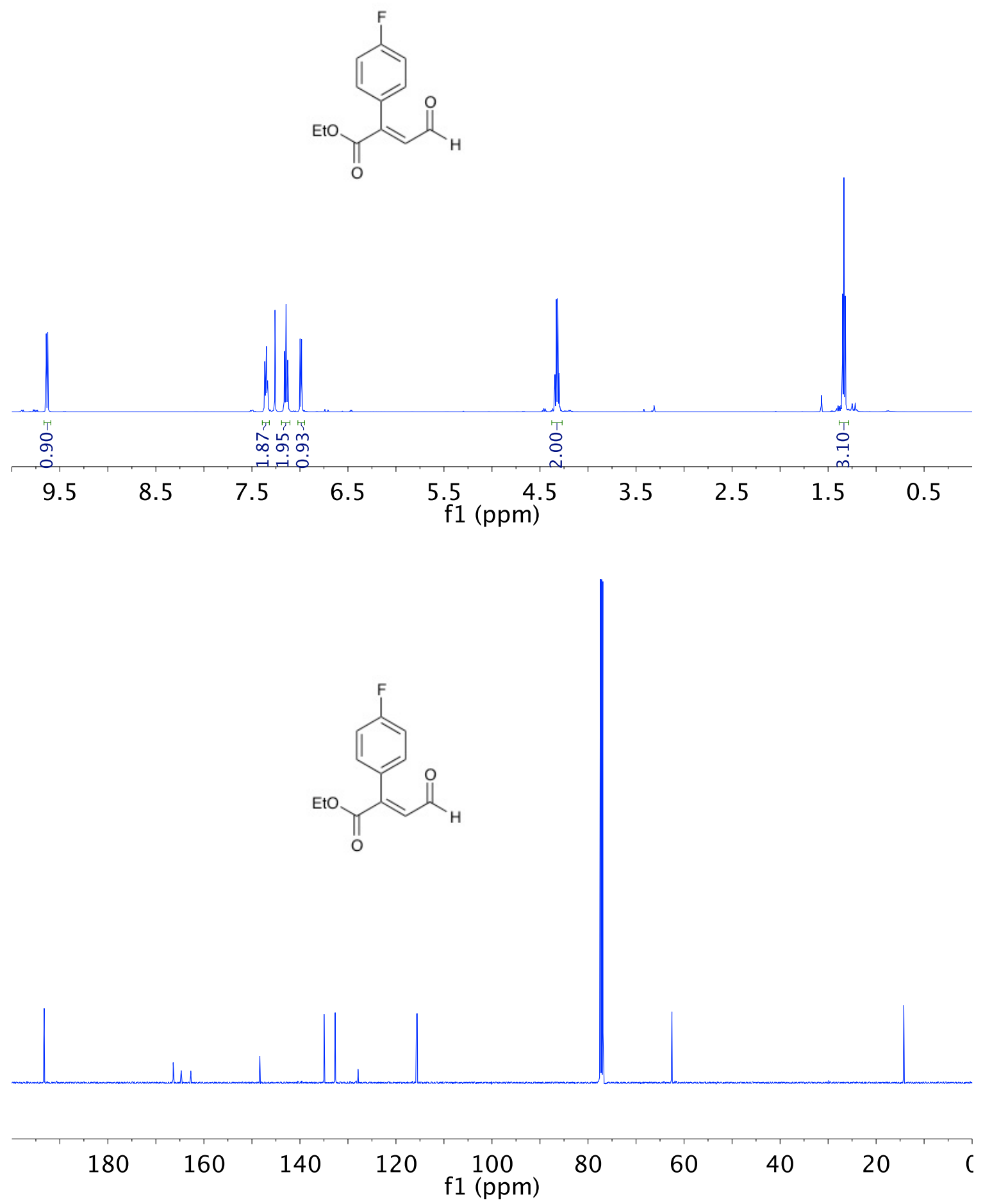

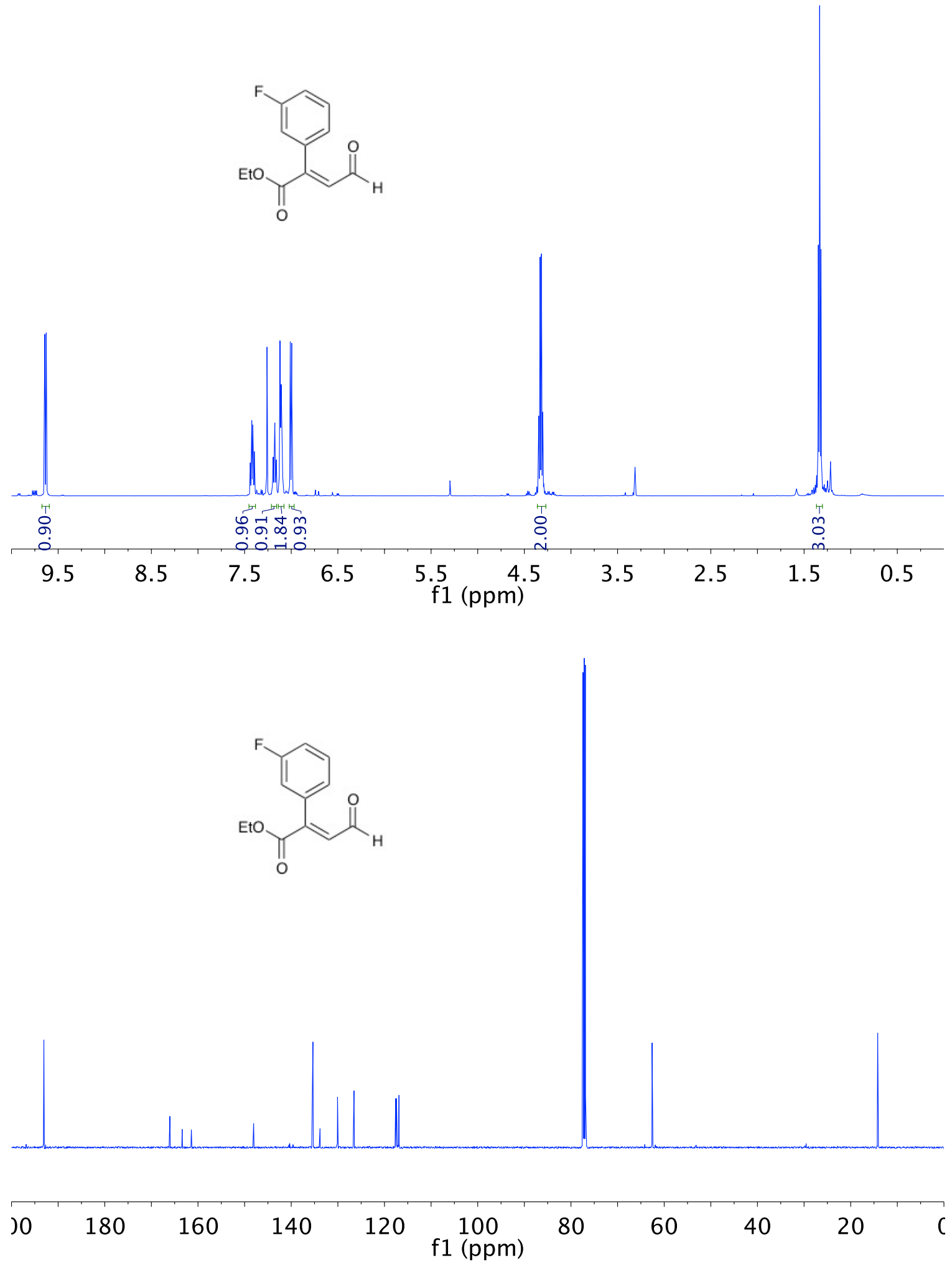

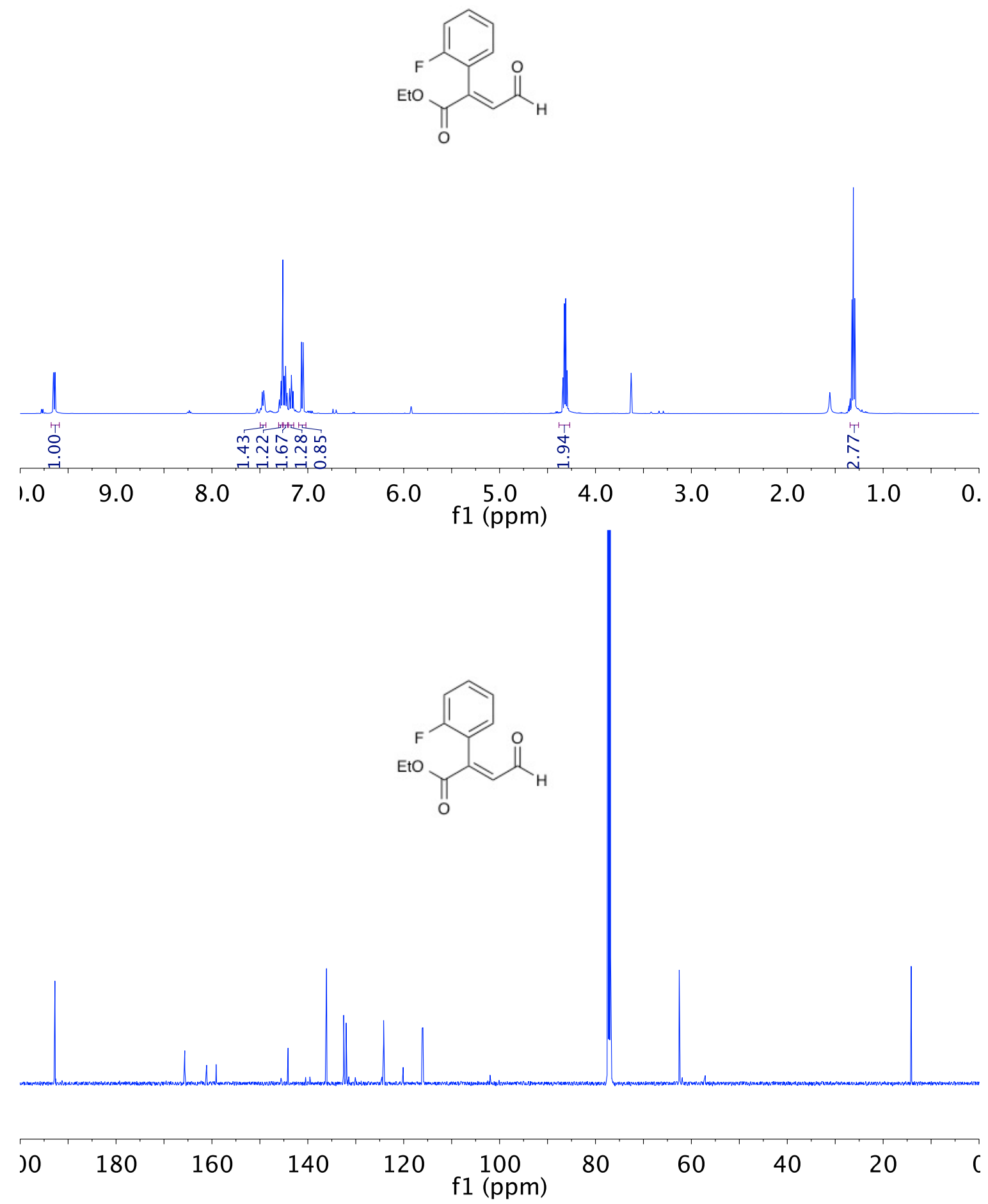

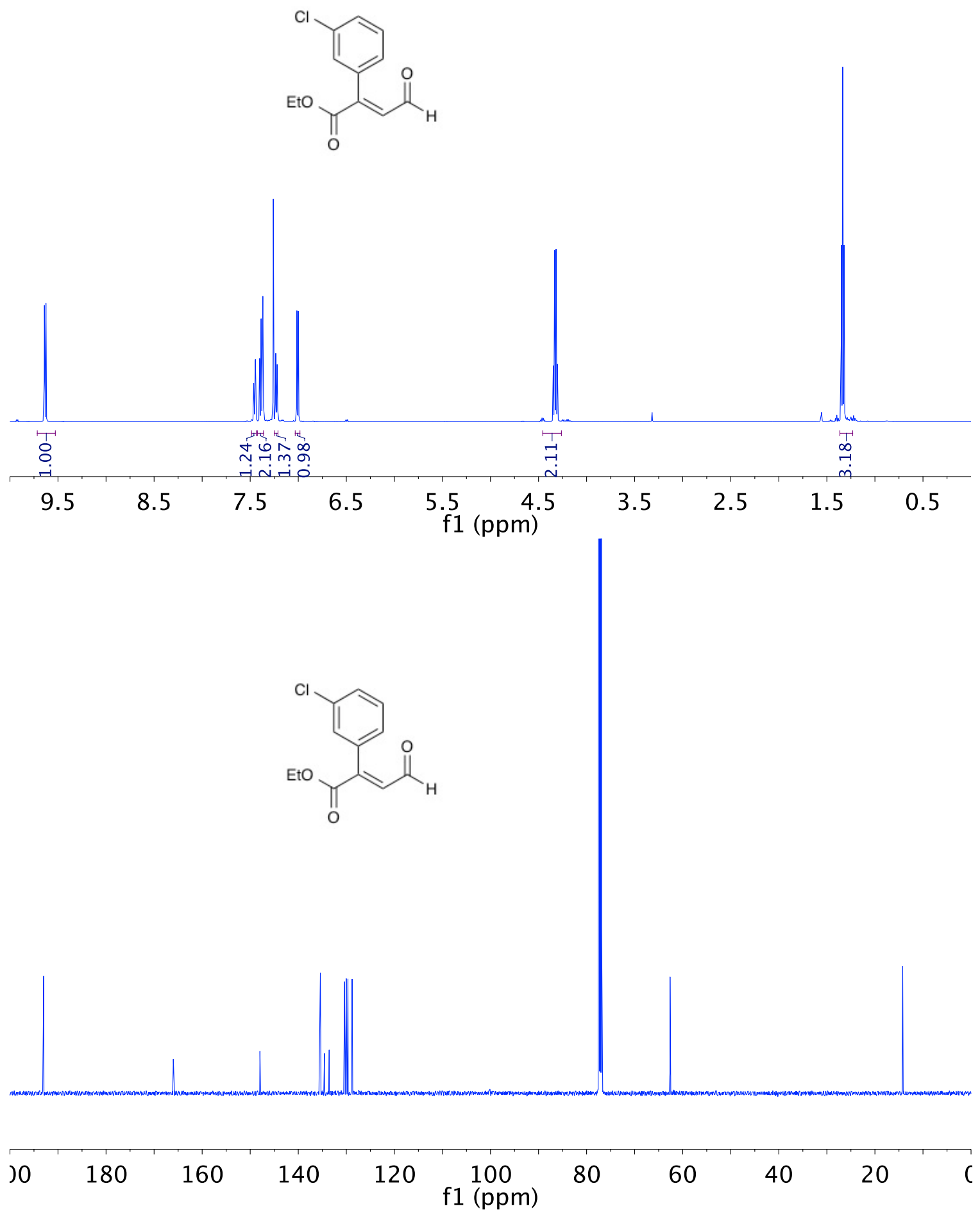

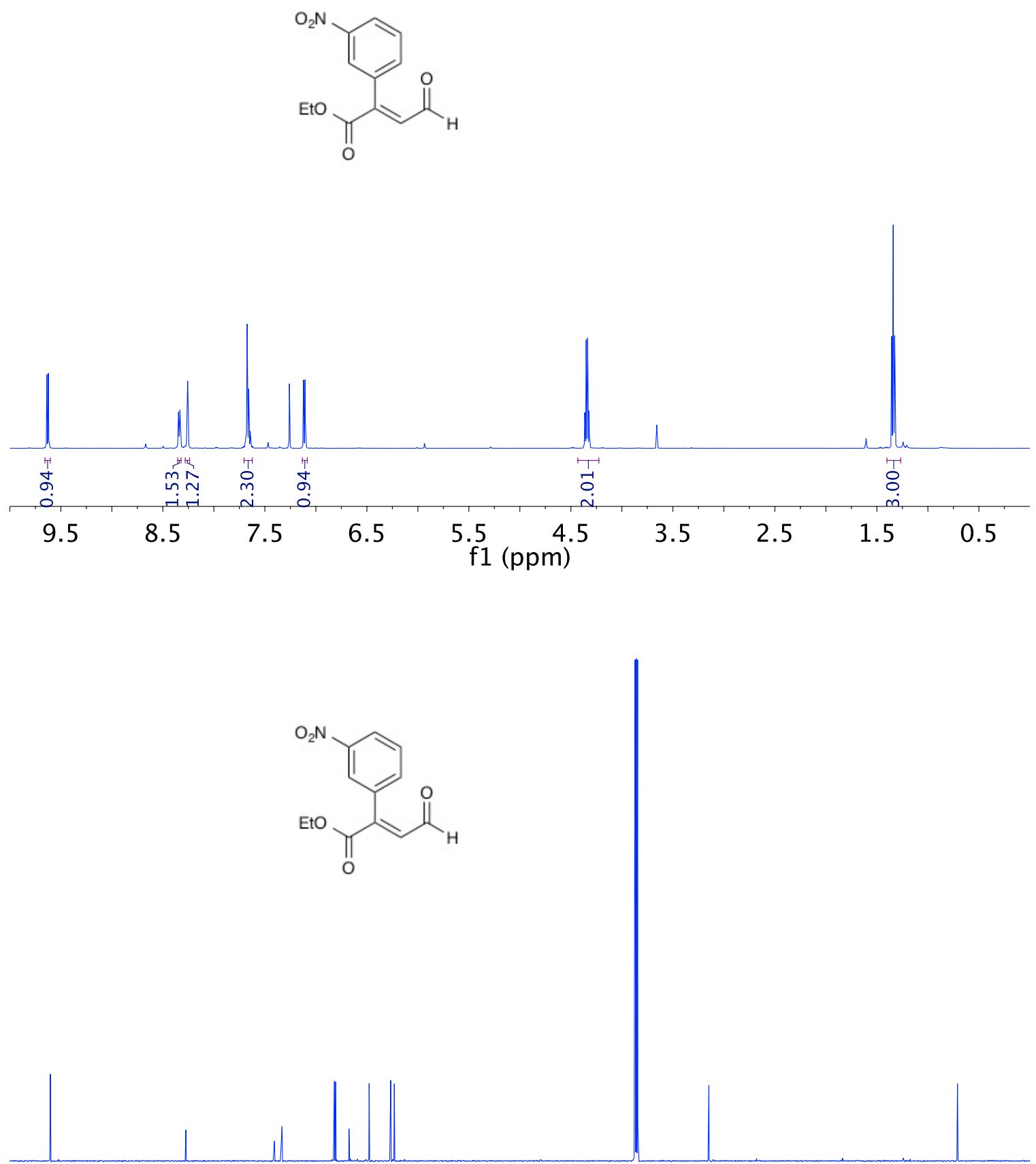

$\begin{array}{lllllllll}180 & 160 & 140 & 120 & \begin{array}{c}100 \\ \mathrm{f} 1 \mathrm{(ppm})\end{array} & 80 & 60 & 40 & 20\end{array}$



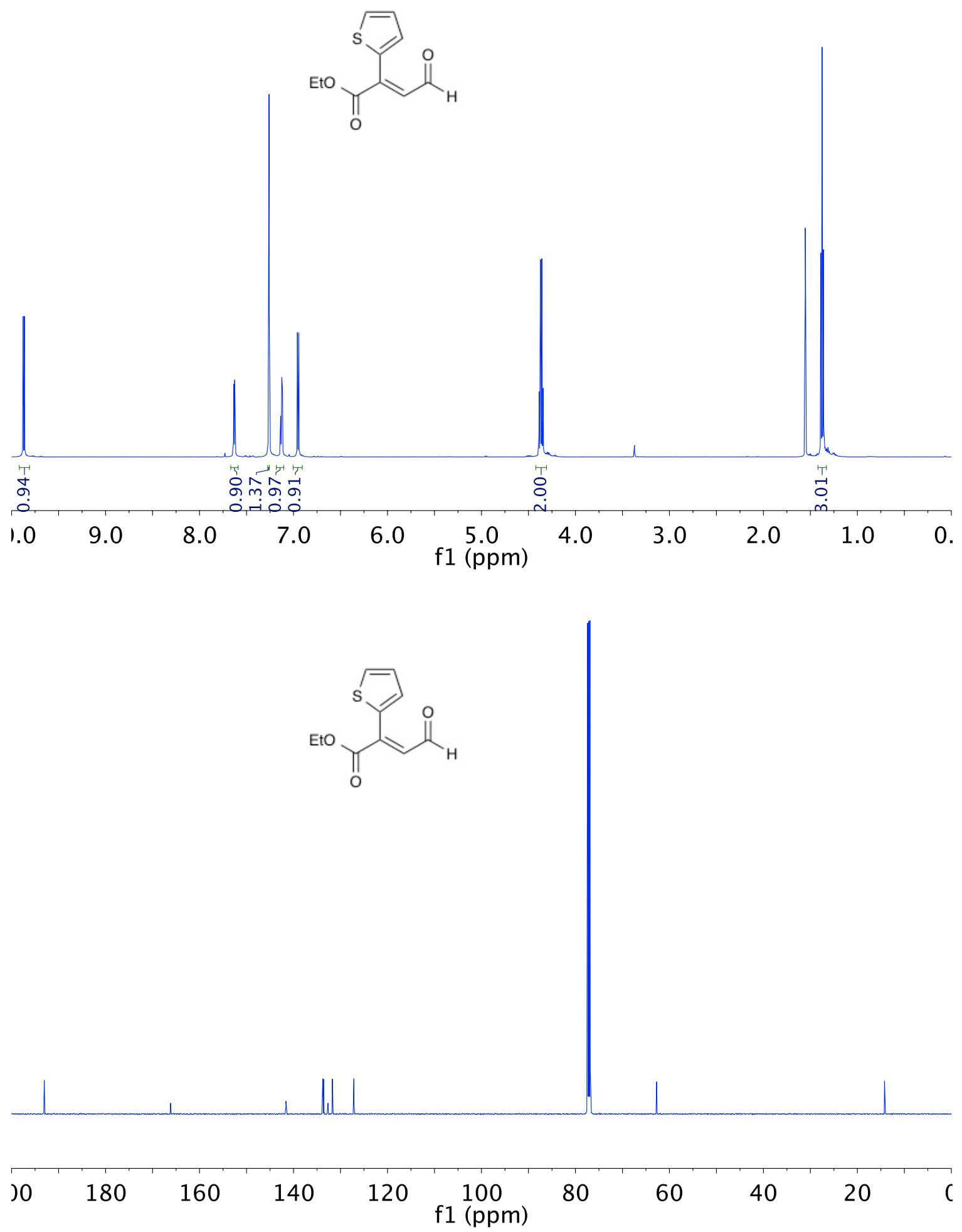

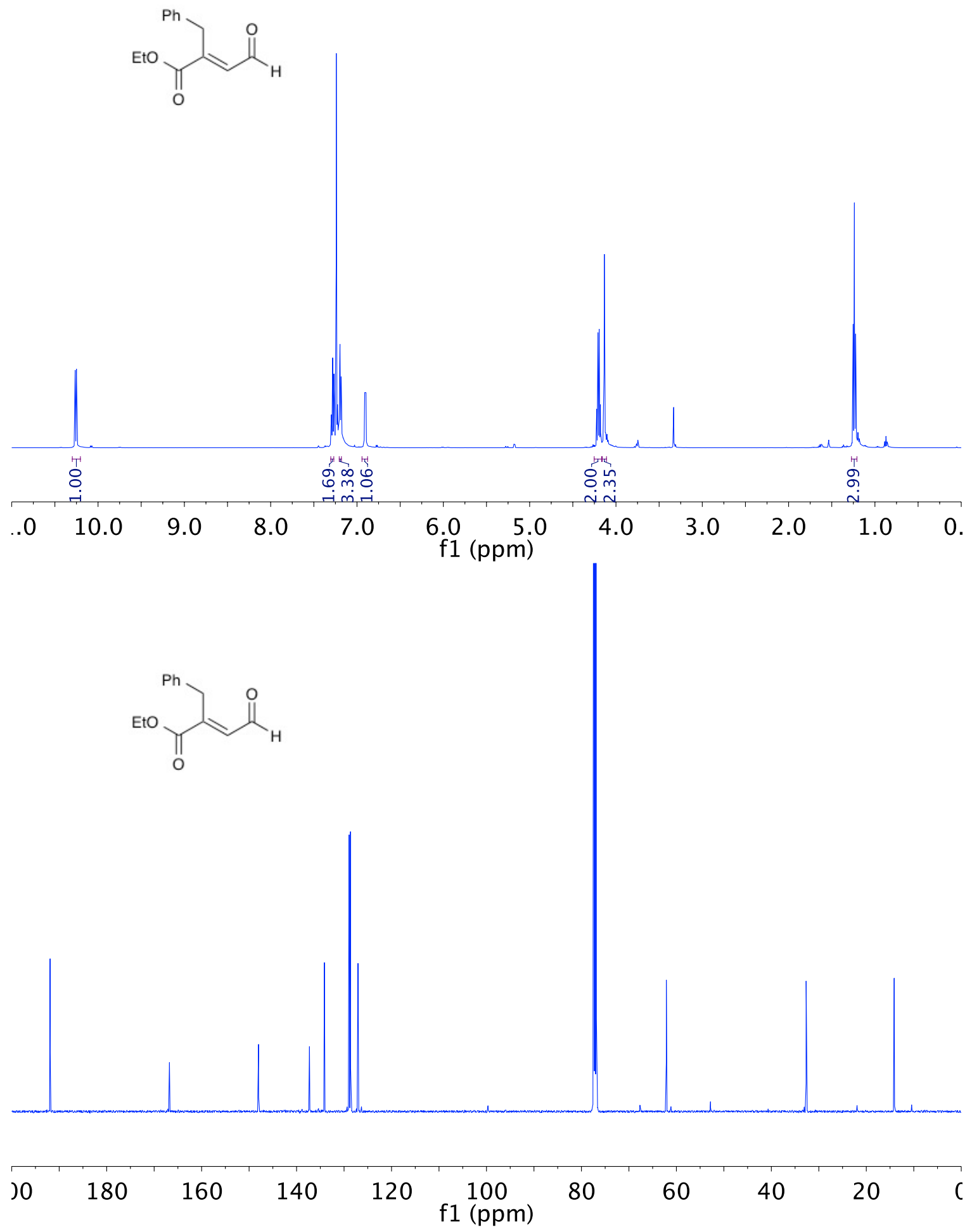

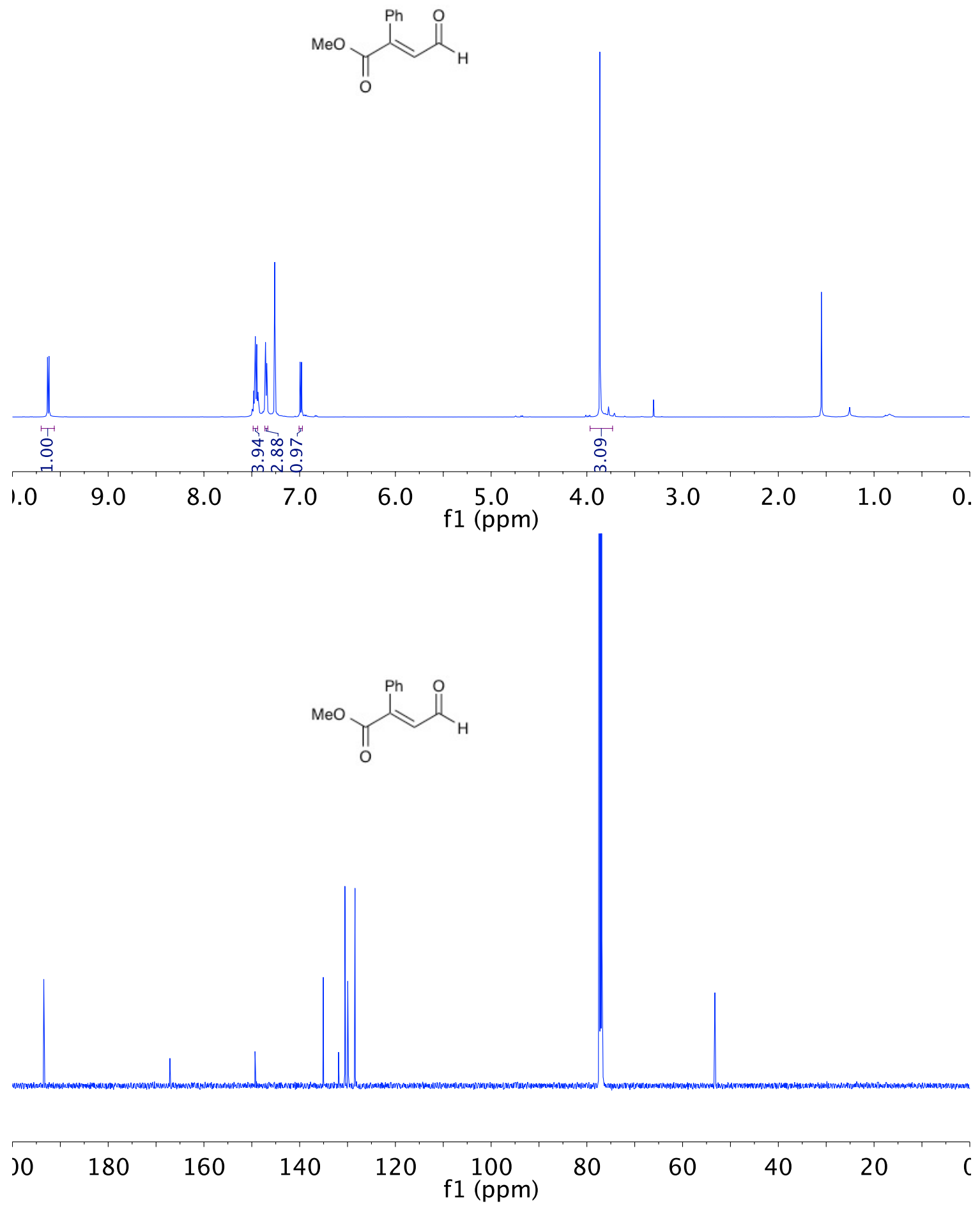

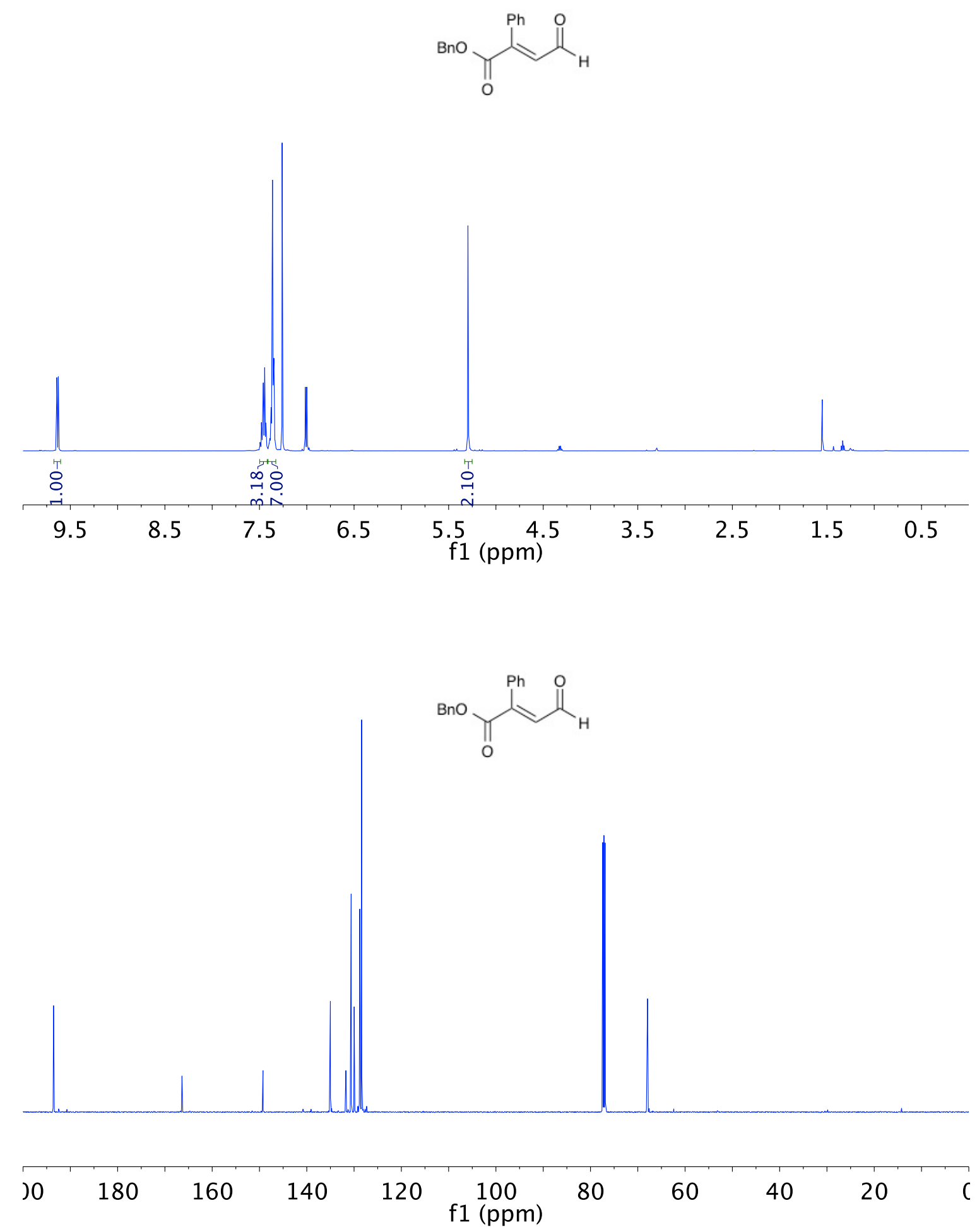

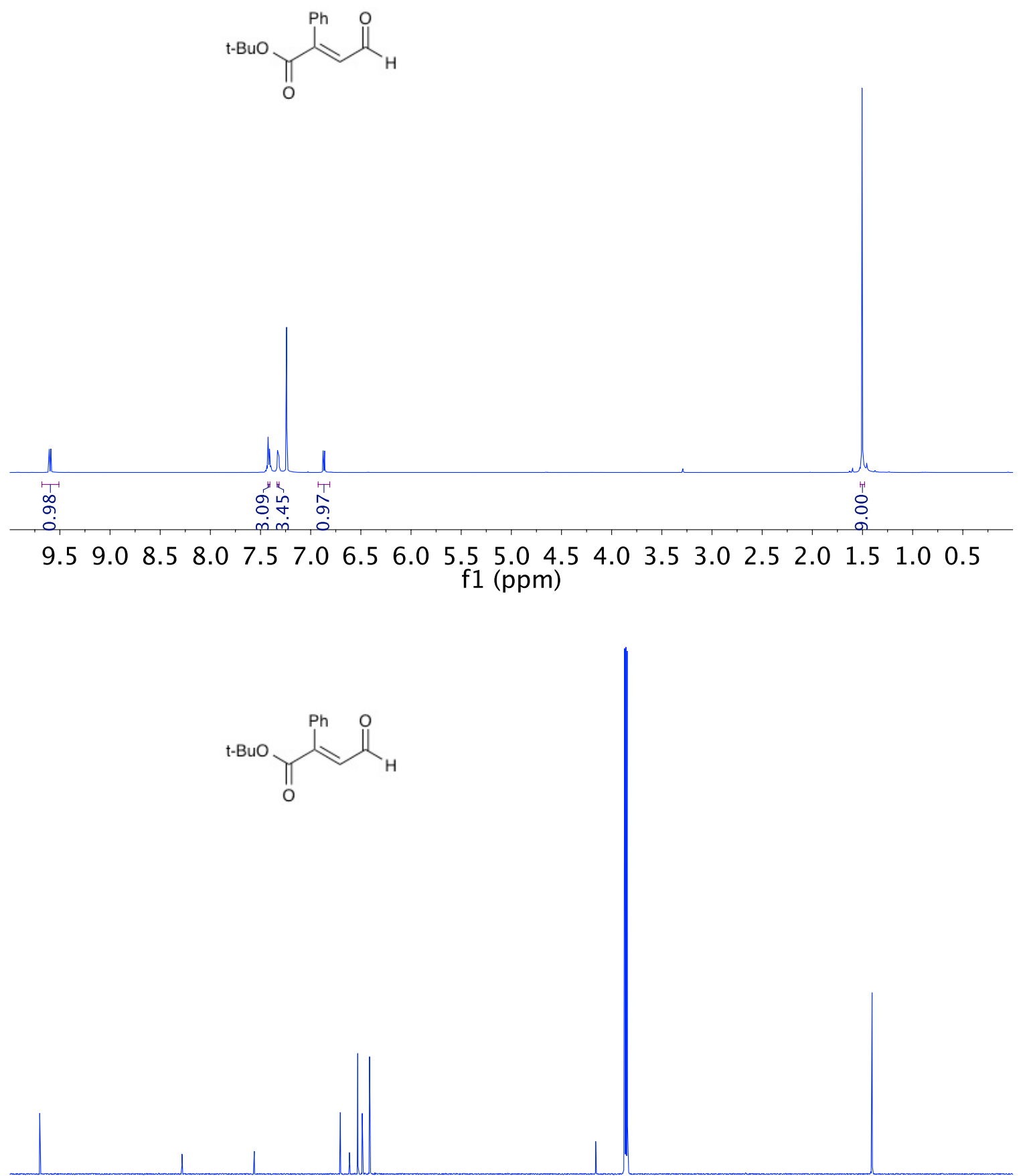

\begin{tabular}{|c|c|c|c|c|c|c|c|c|c|}
\hline$) 0$ & 180 & 160 & 140 & 120 & $\begin{array}{l}100 \\
\mathrm{f} 1 \text { (ppm) }\end{array}$ & 80 & 60 & 40 & 20 \\
\hline
\end{tabular}



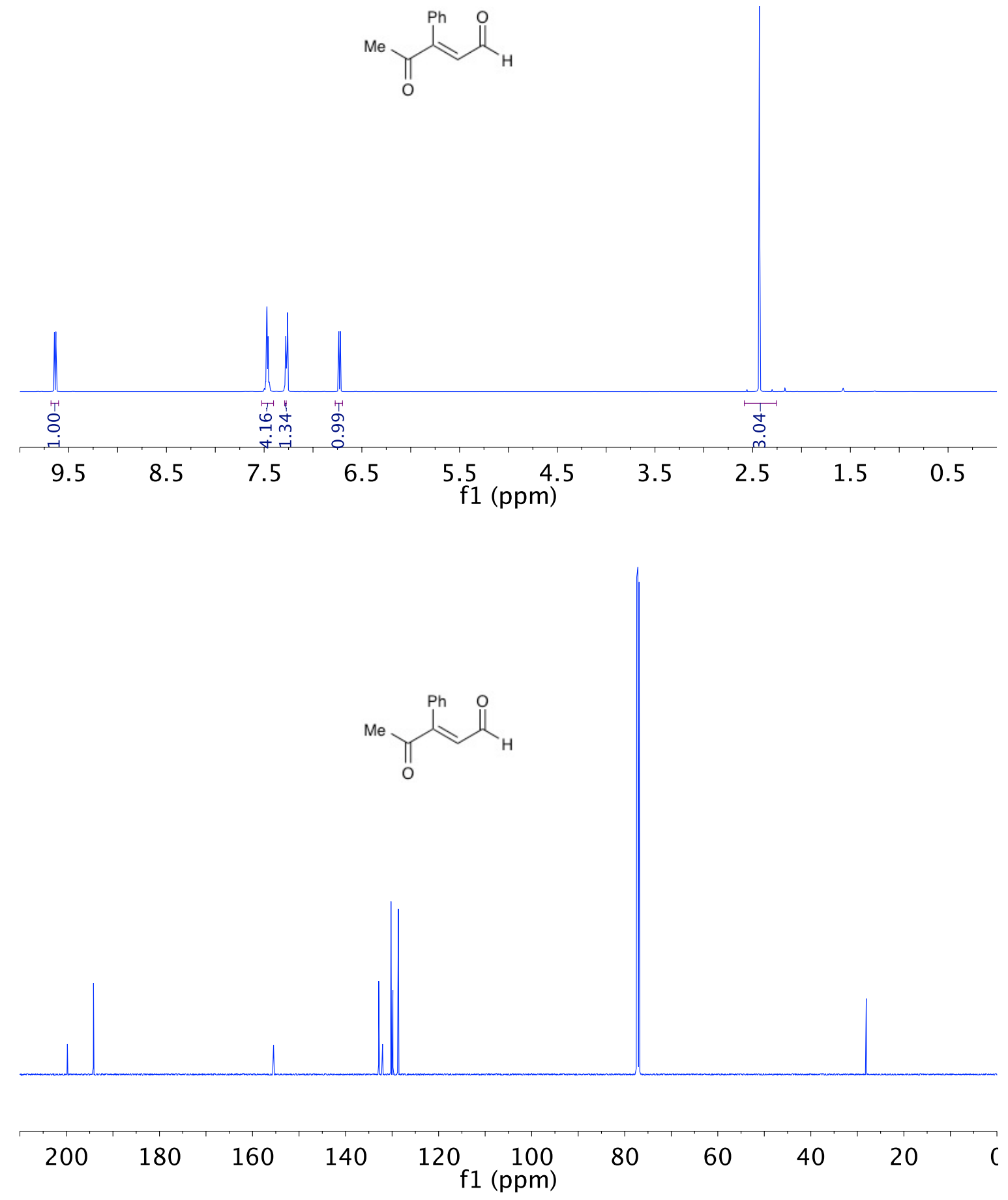

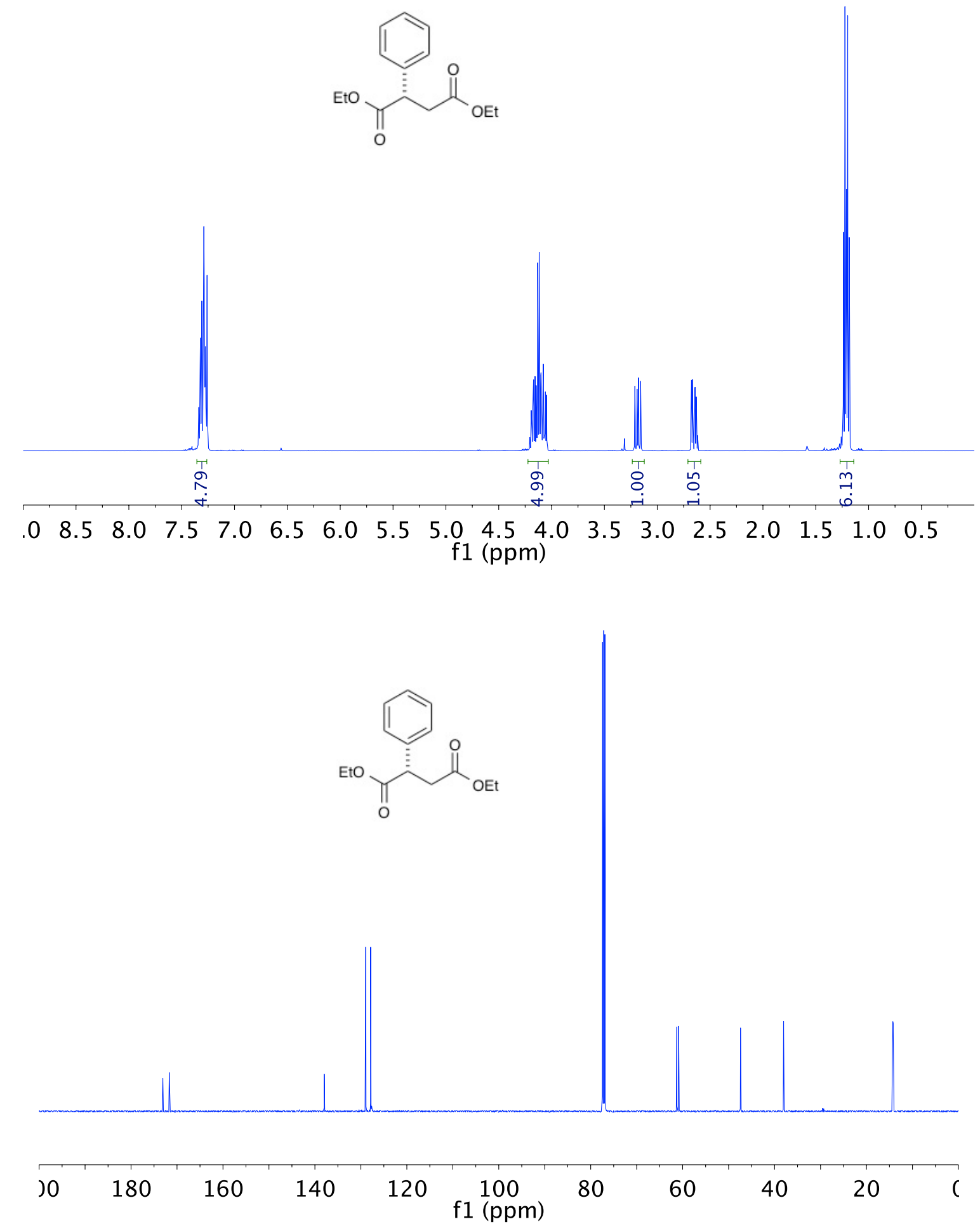

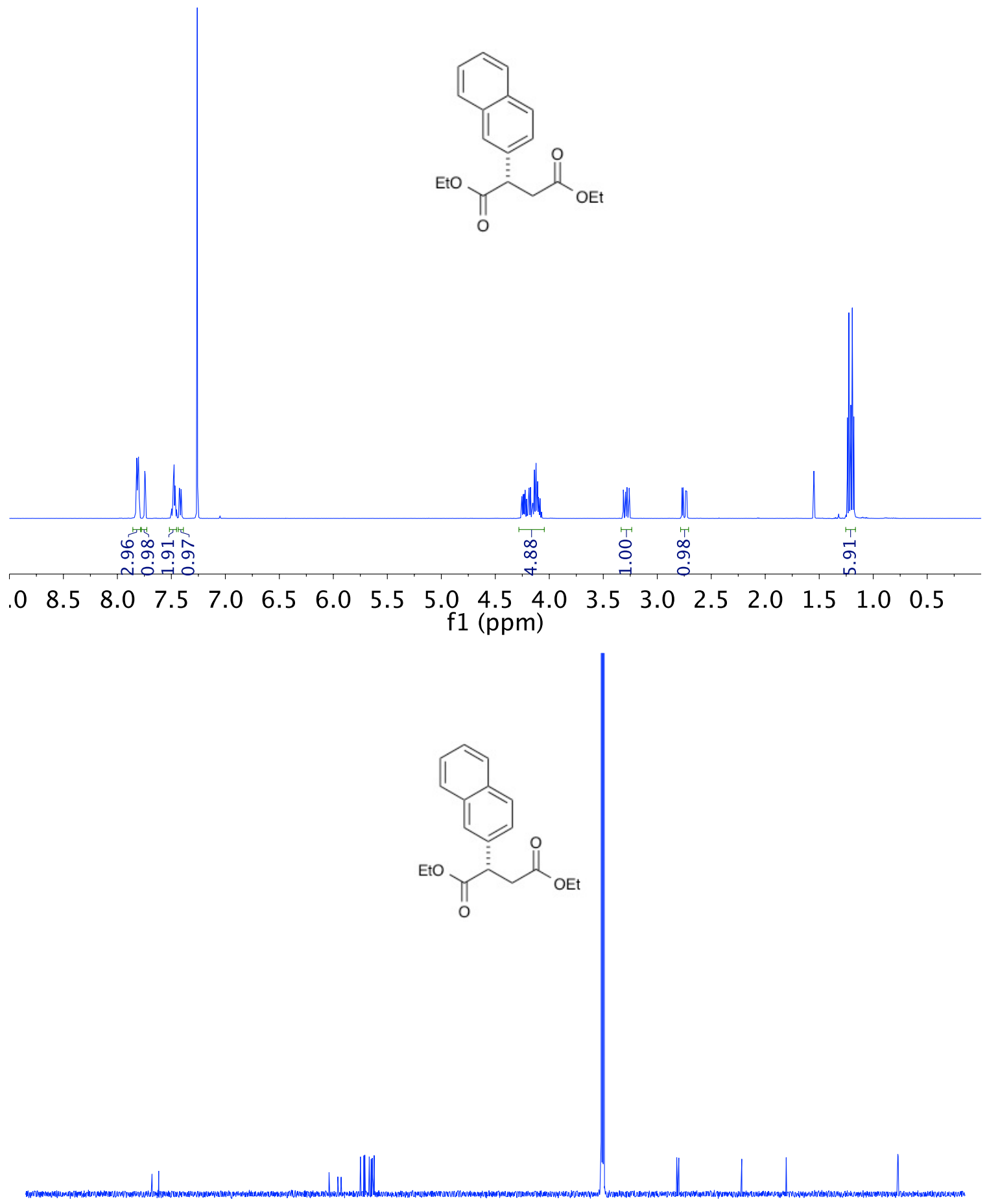

) $\left.0180 \quad 160 \quad 140 \quad 120 \begin{array}{c}100 \\ \mathrm{f} 1(\mathrm{ppm})\end{array}\right)$



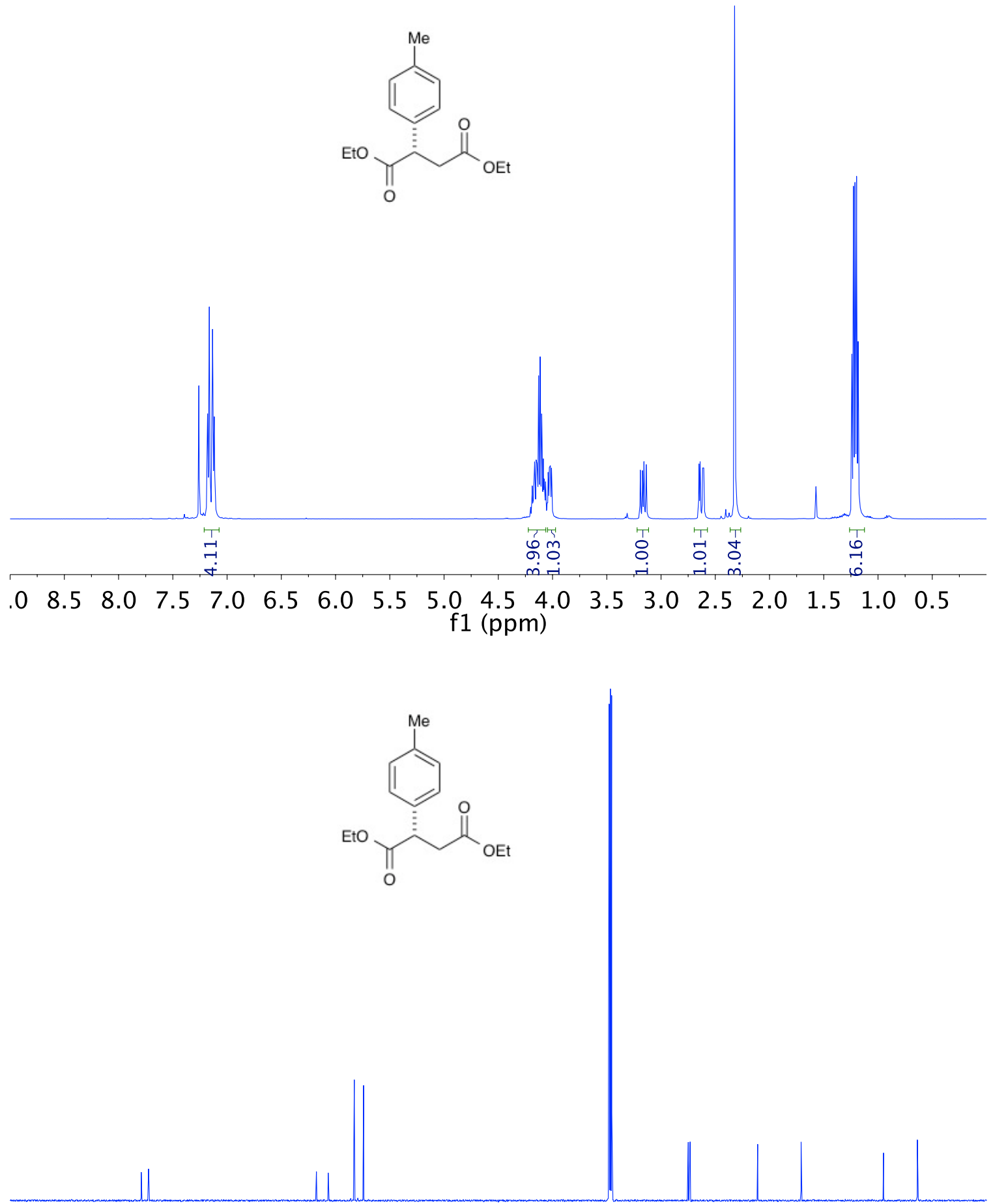

\begin{tabular}{|c|c|c|c|c|c|c|c|c|c|}
\hline$) 0$ & 180 & 160 & 140 & 120 & $\begin{array}{c}100 \\
\mathrm{f} 1(\mathrm{ppm})\end{array}$ & 80 & 60 & 40 & 20 \\
\hline
\end{tabular}



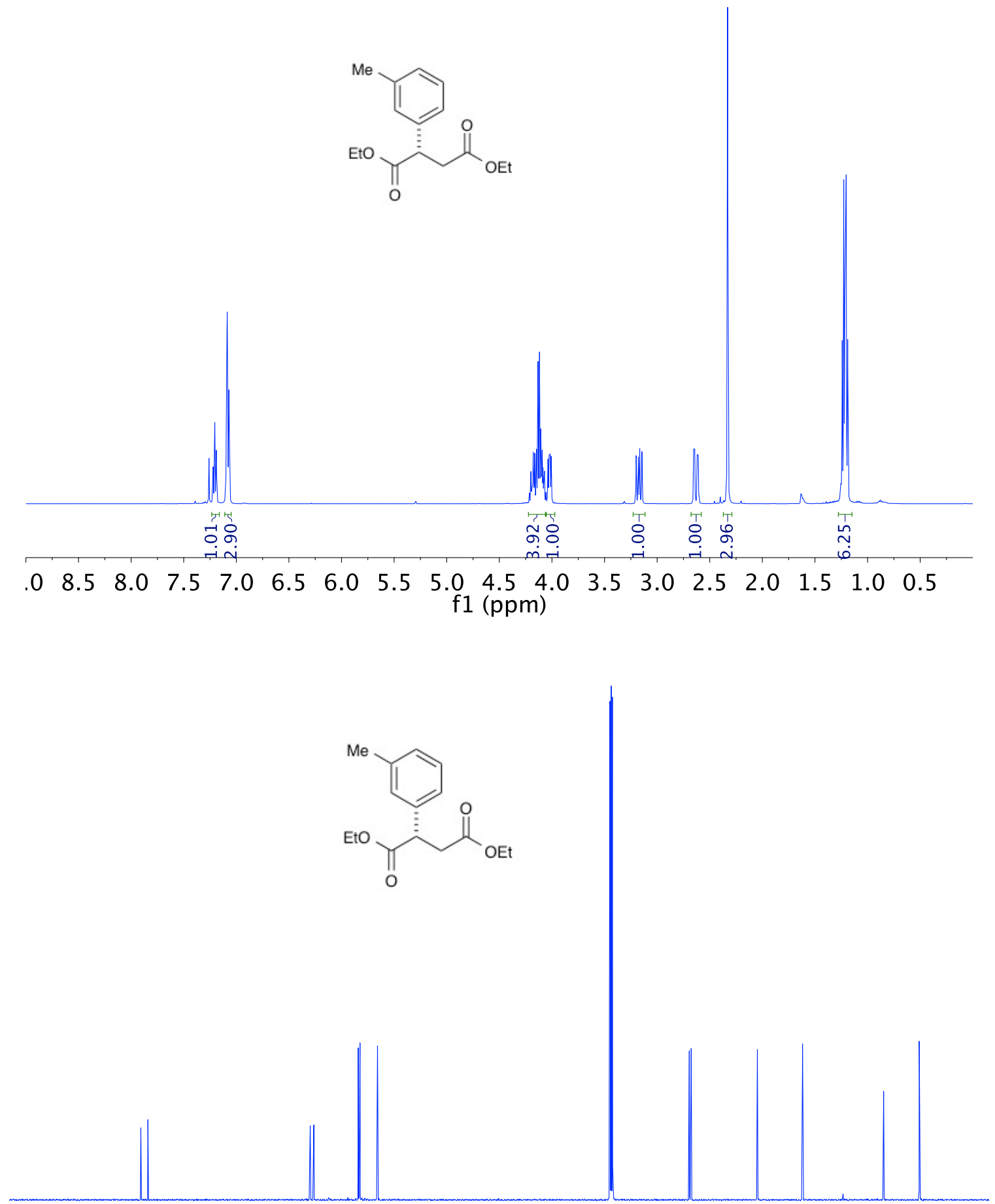

) $\left.0180 \quad 160 \quad 140 \quad 120 \begin{array}{c}100 \\ \mathrm{f} 1(\mathrm{ppm})\end{array}\right)$



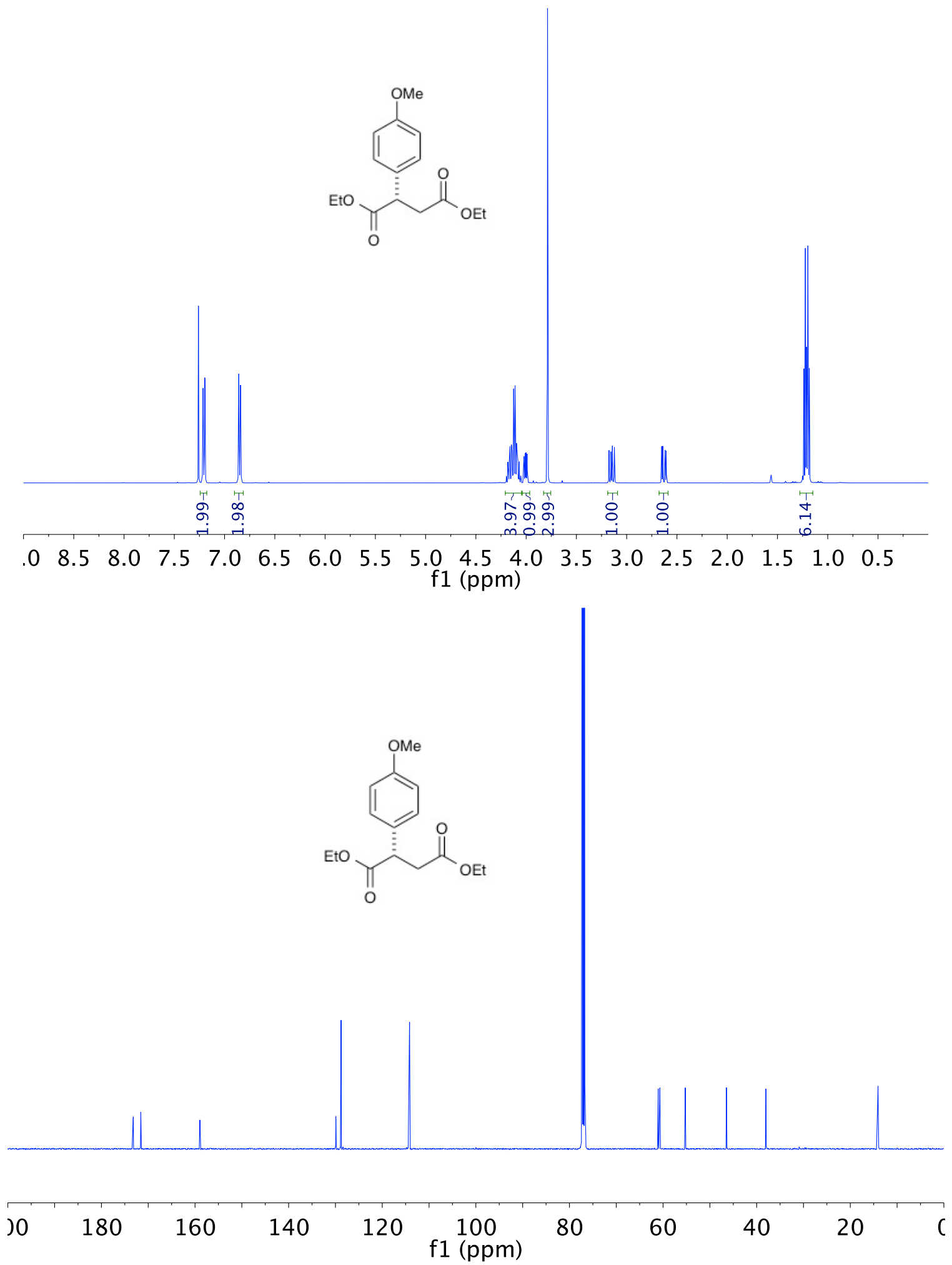

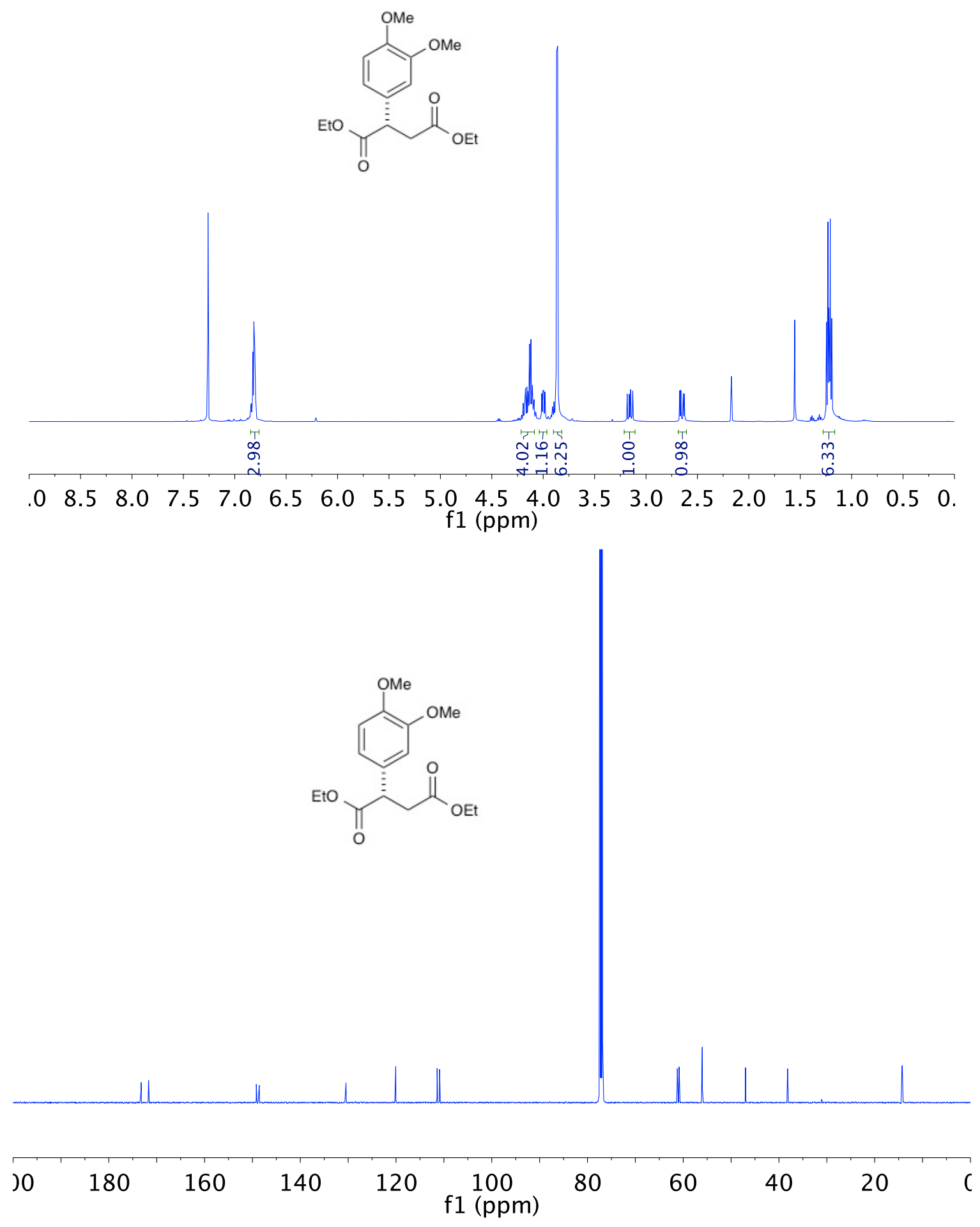

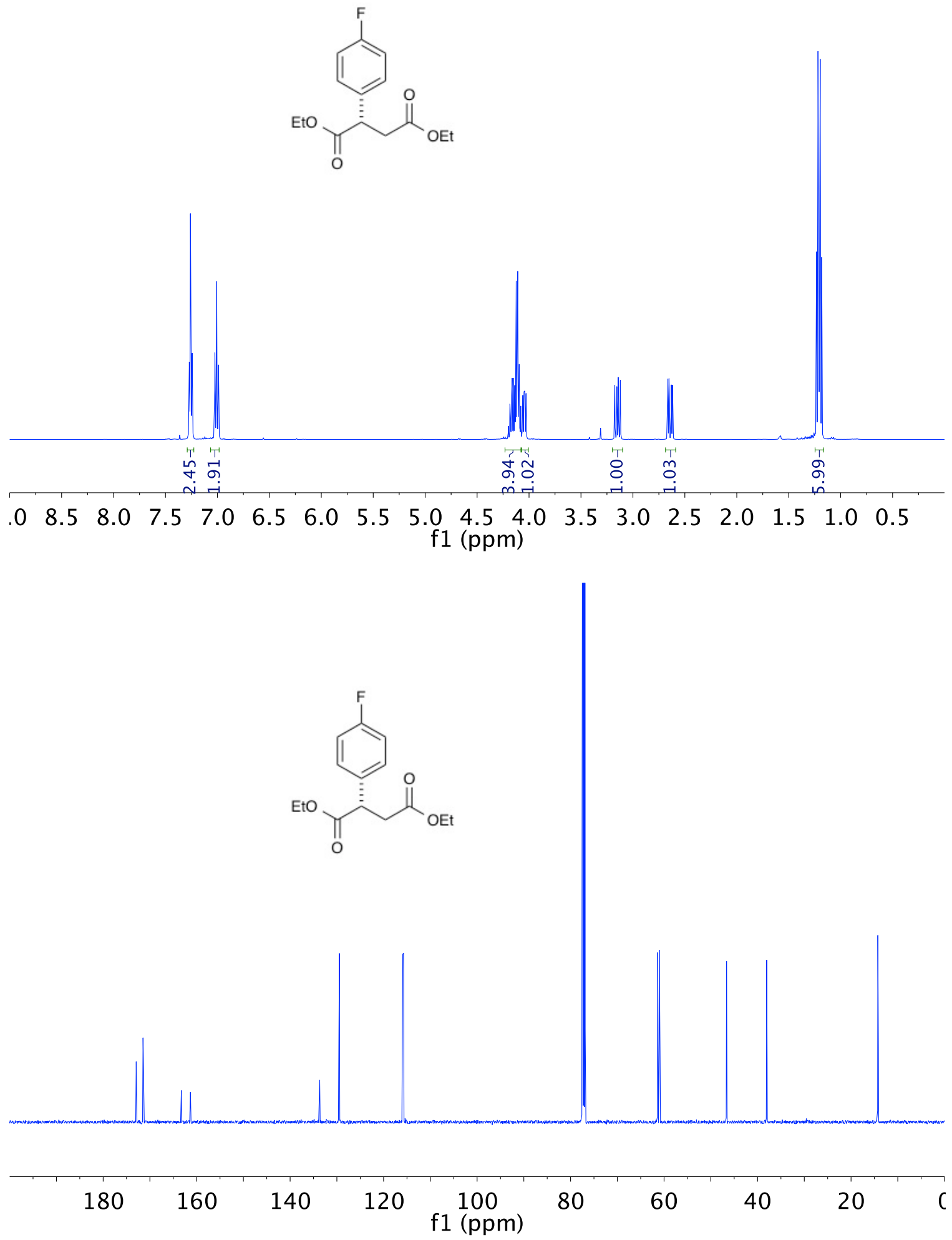

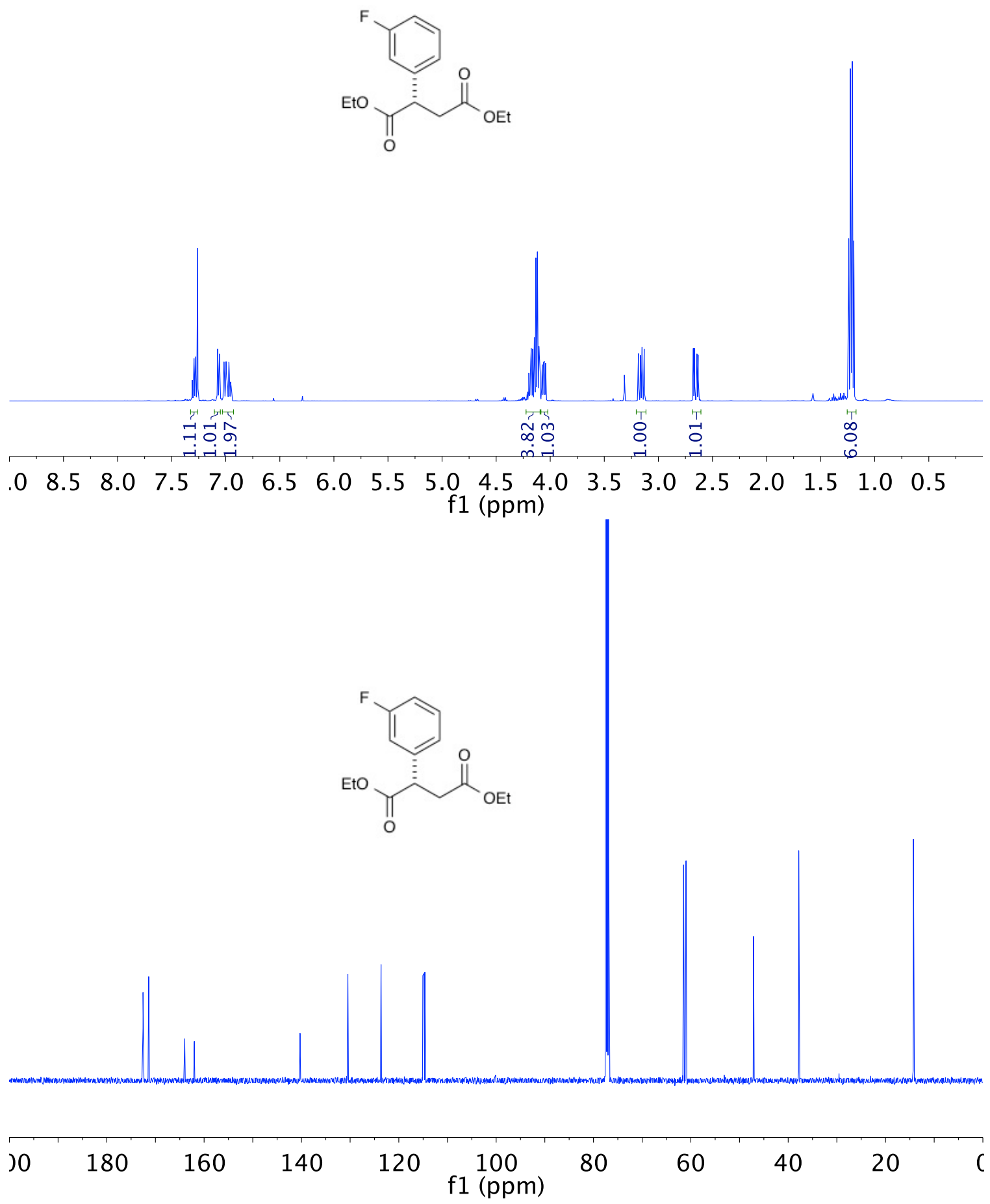

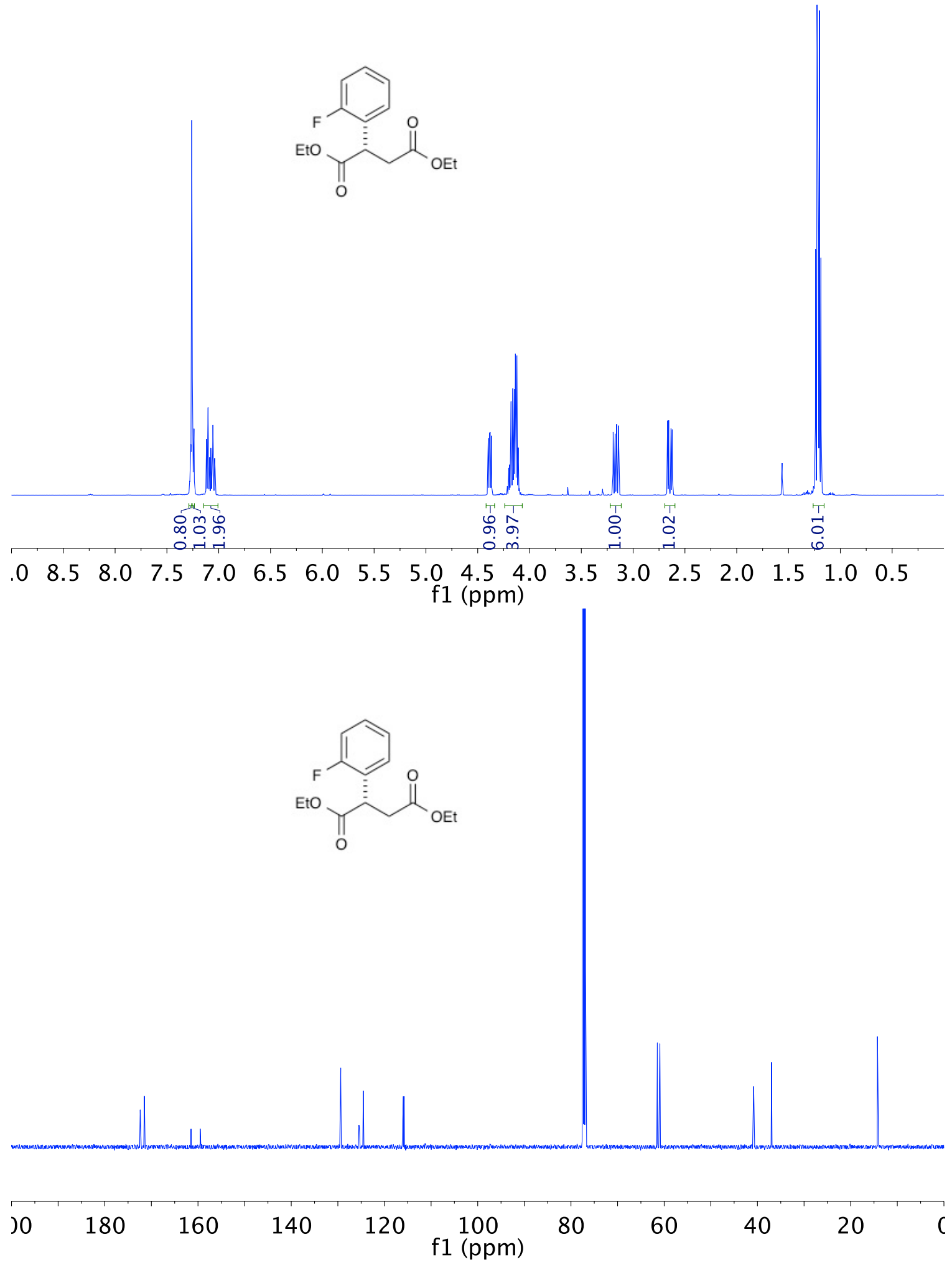

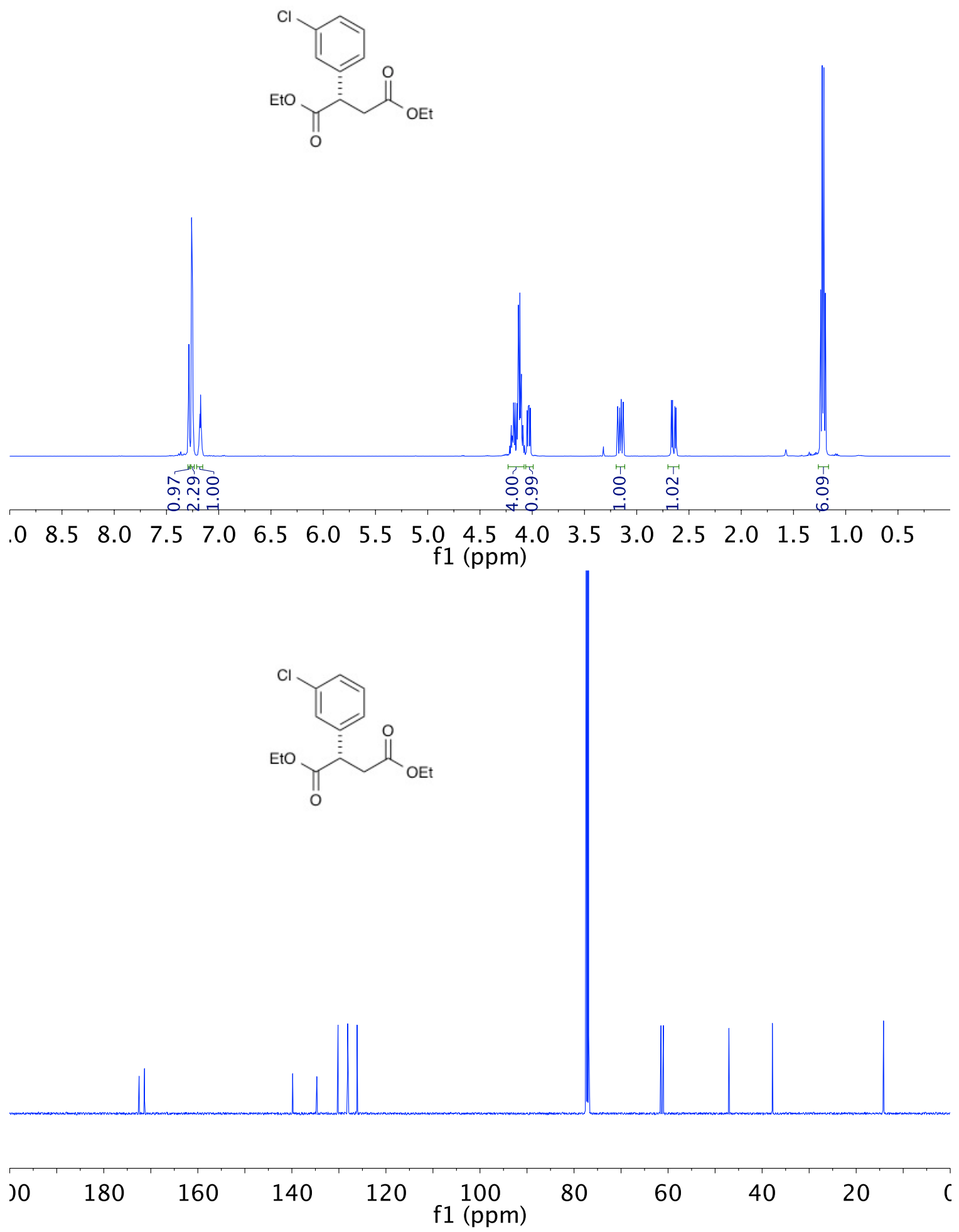

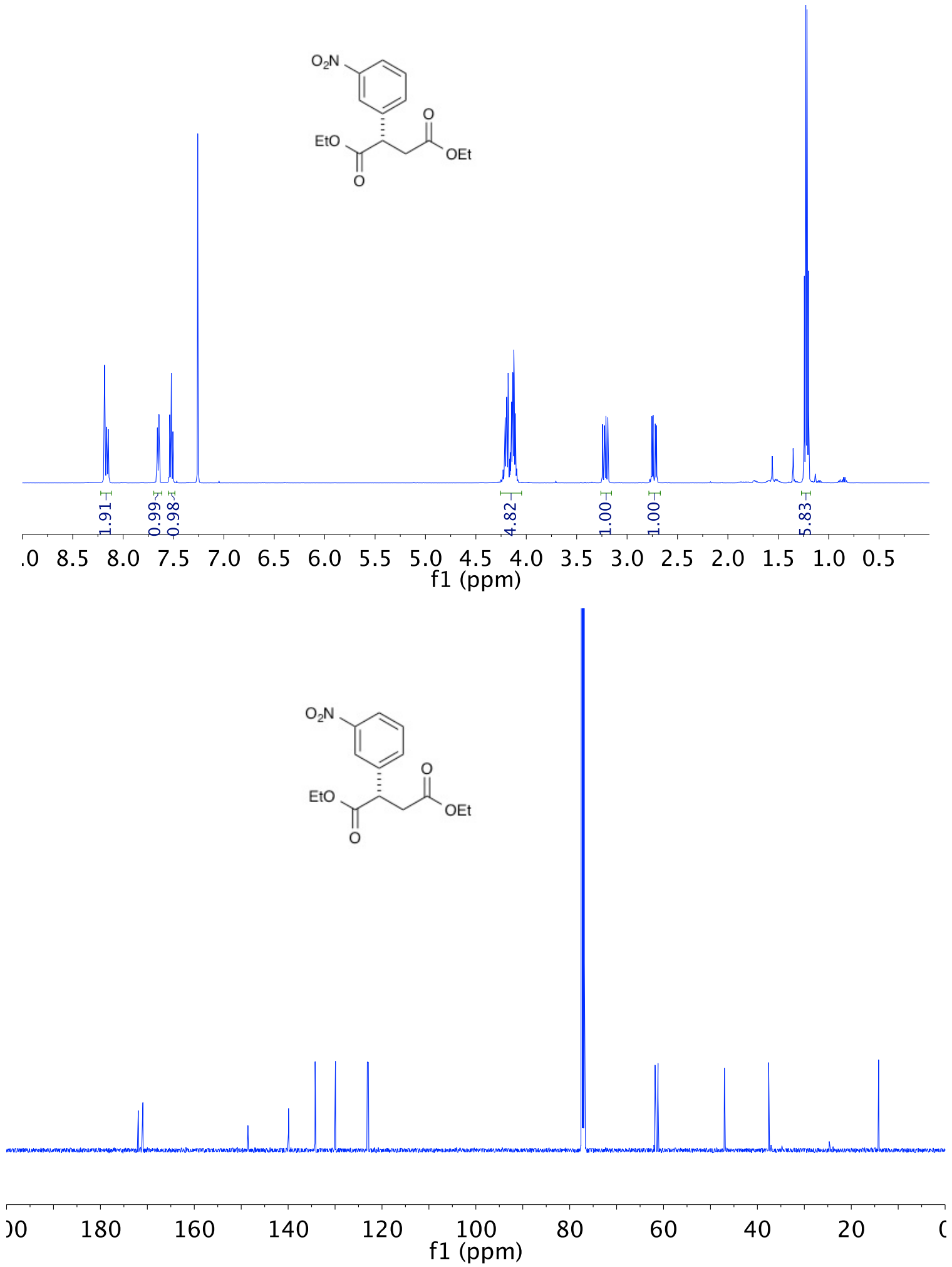


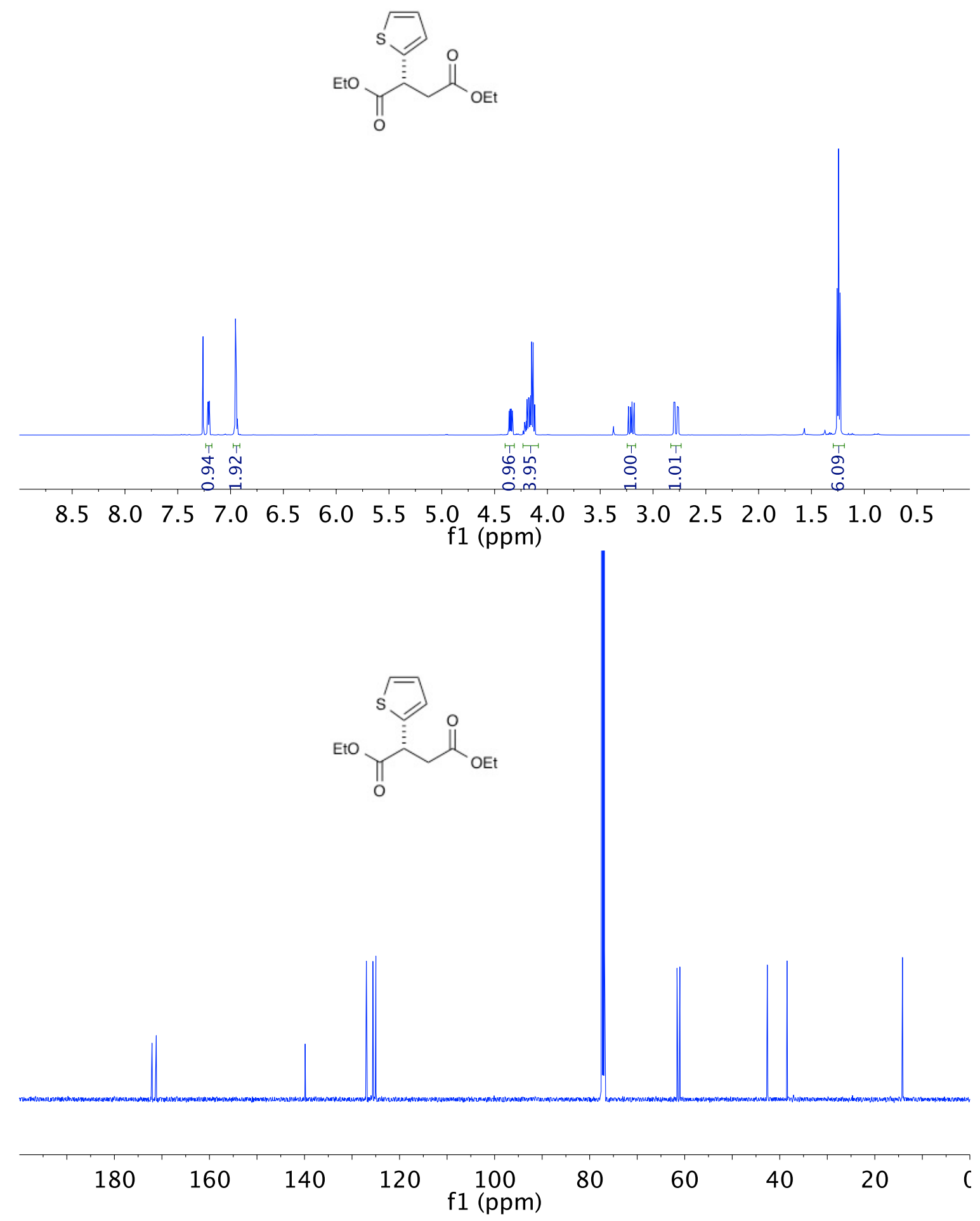



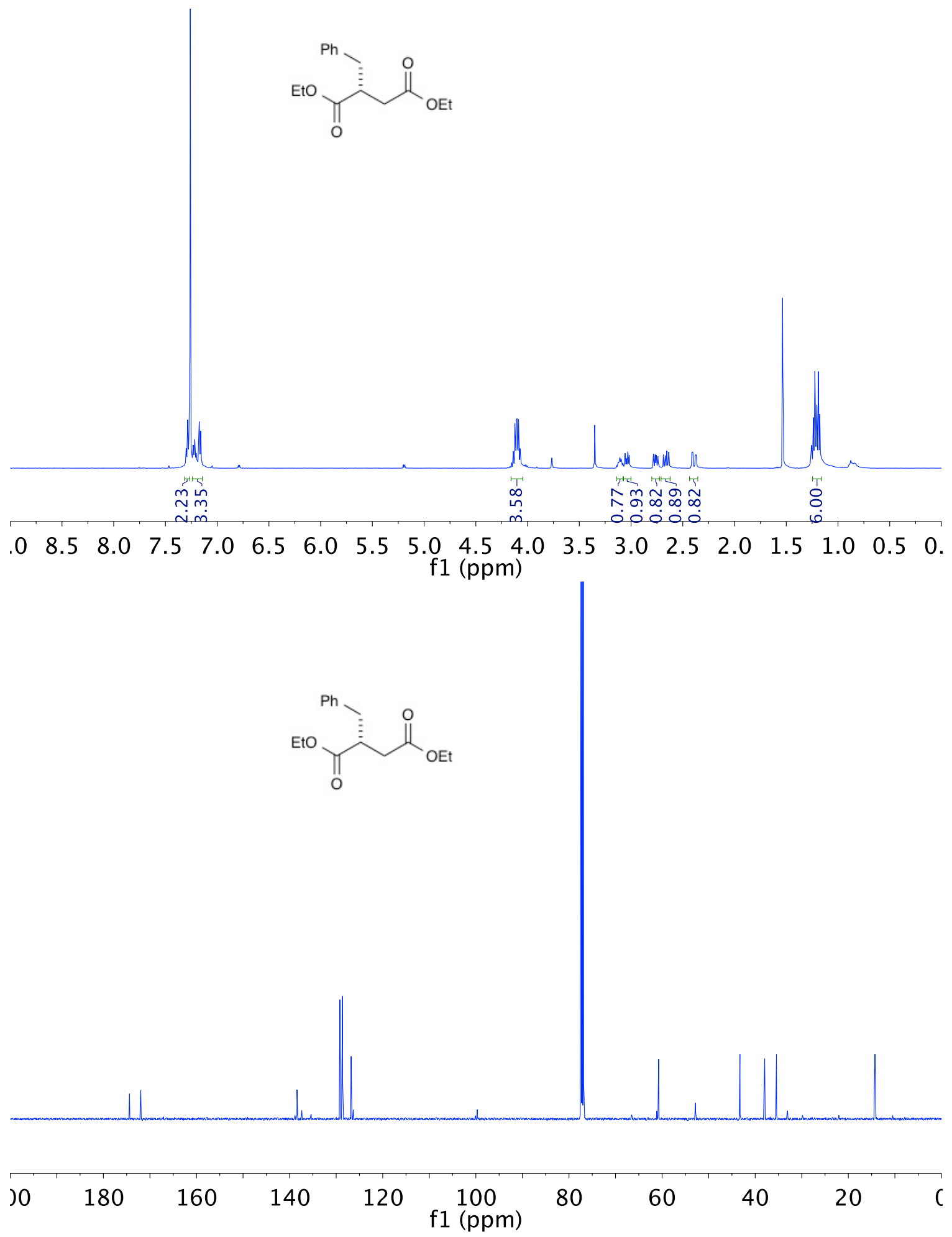

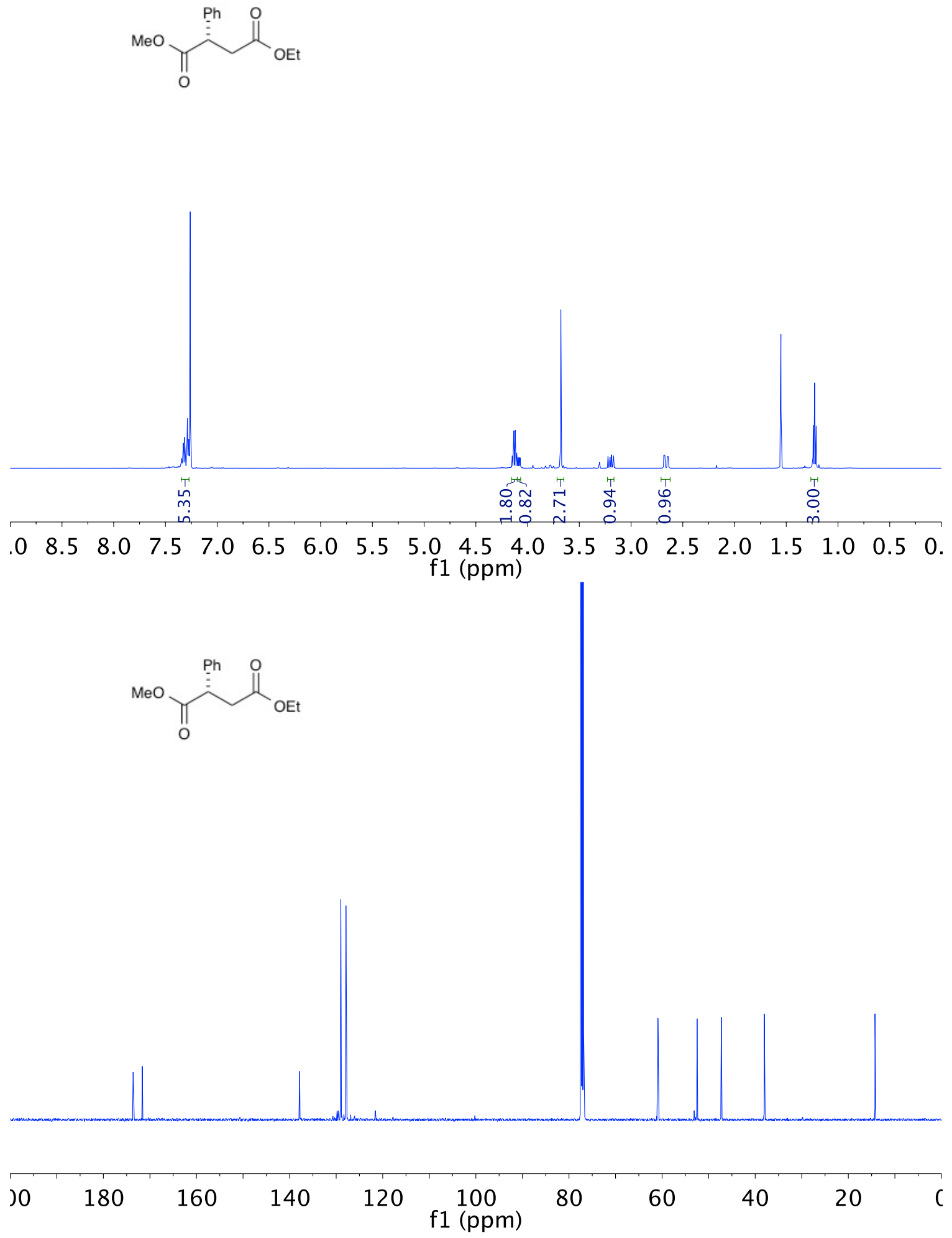


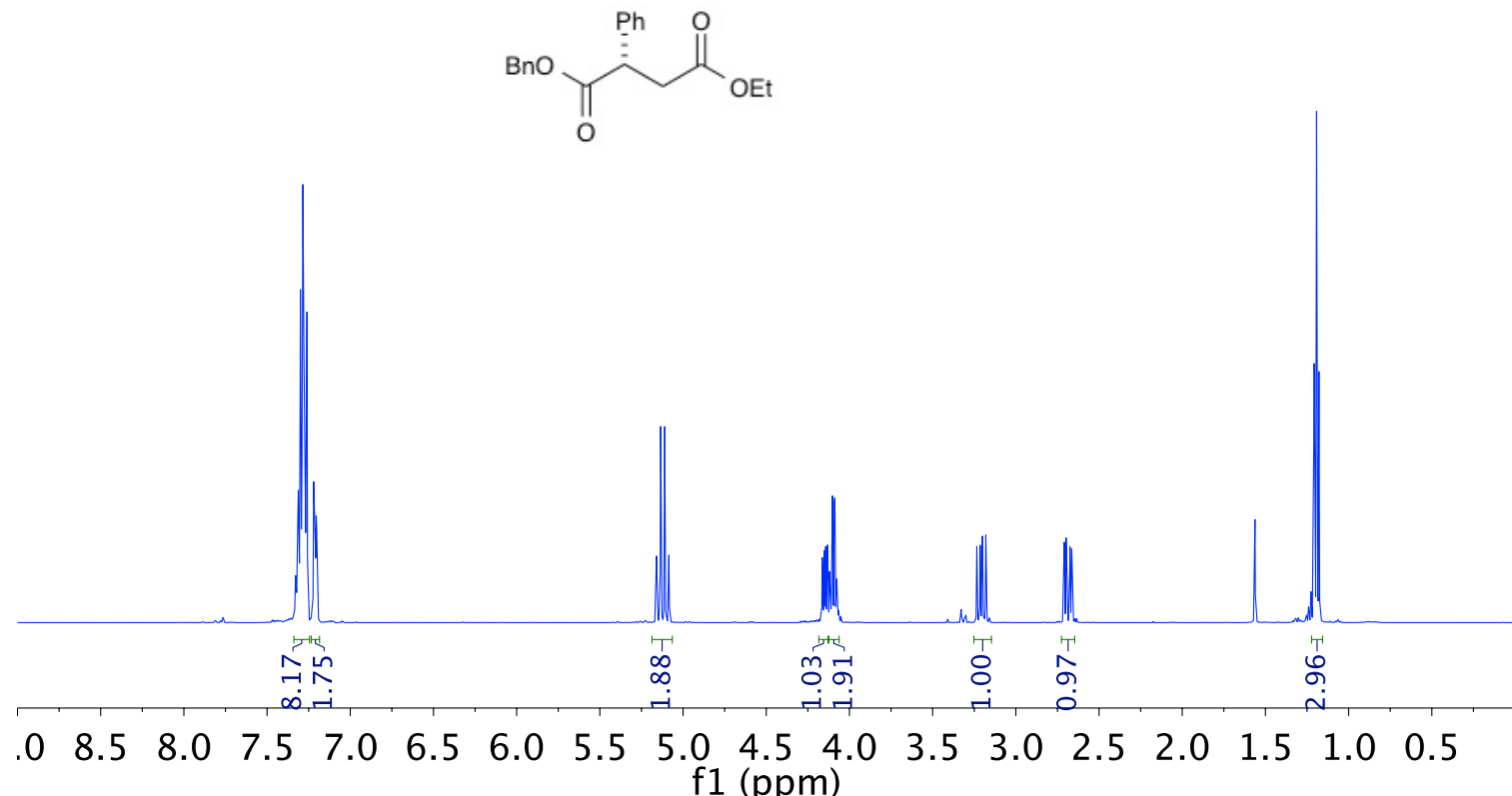
f1 (ppm)
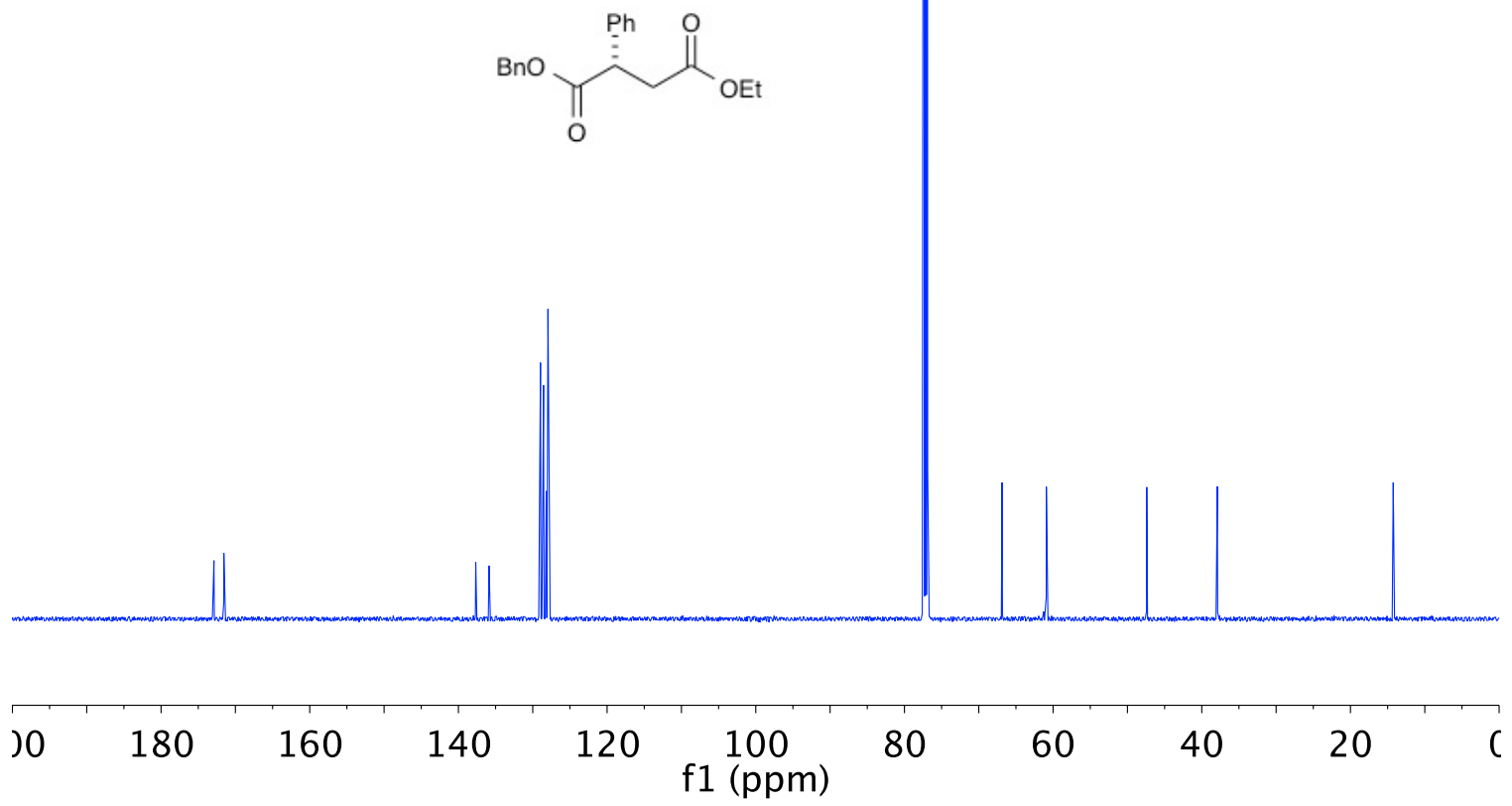

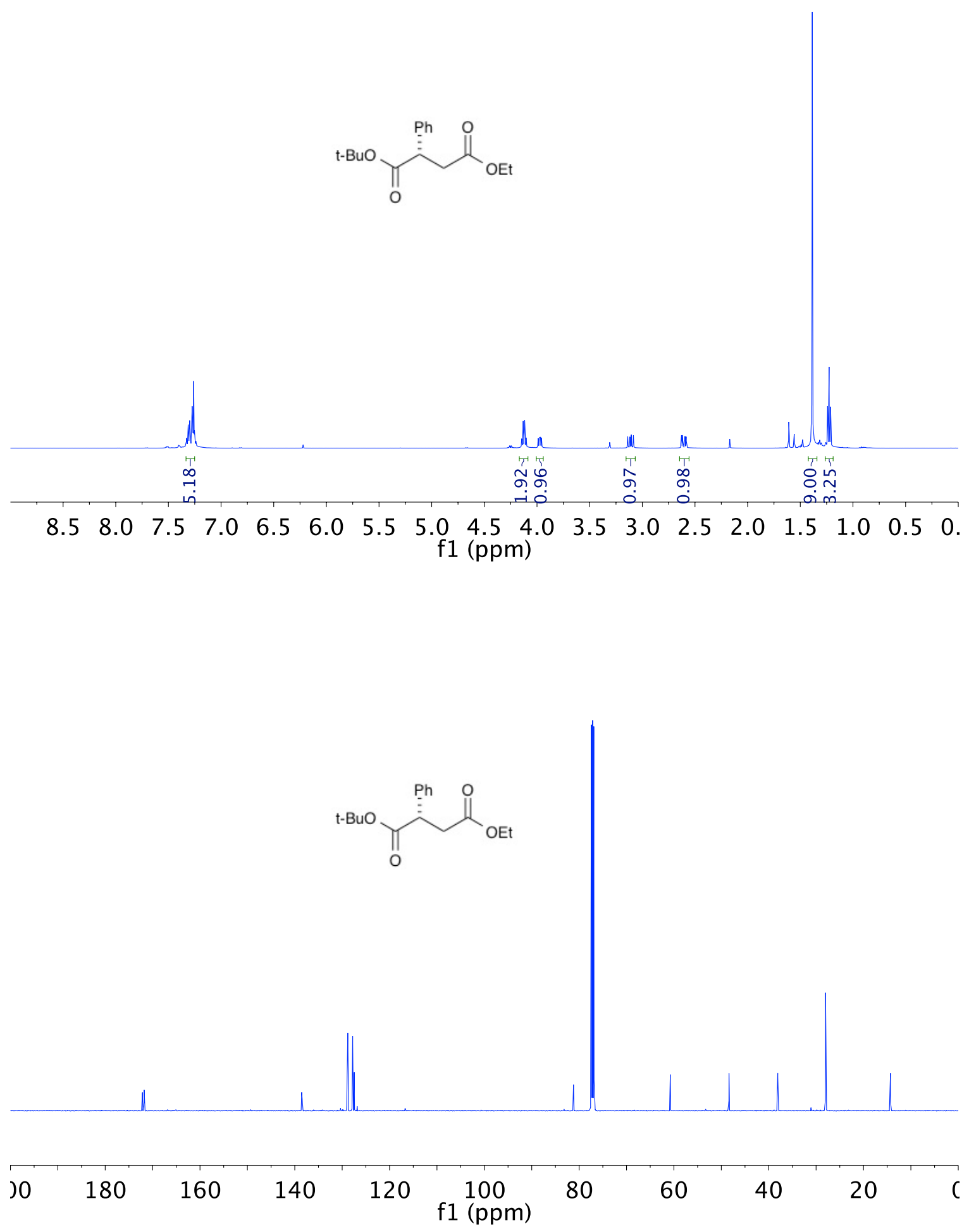


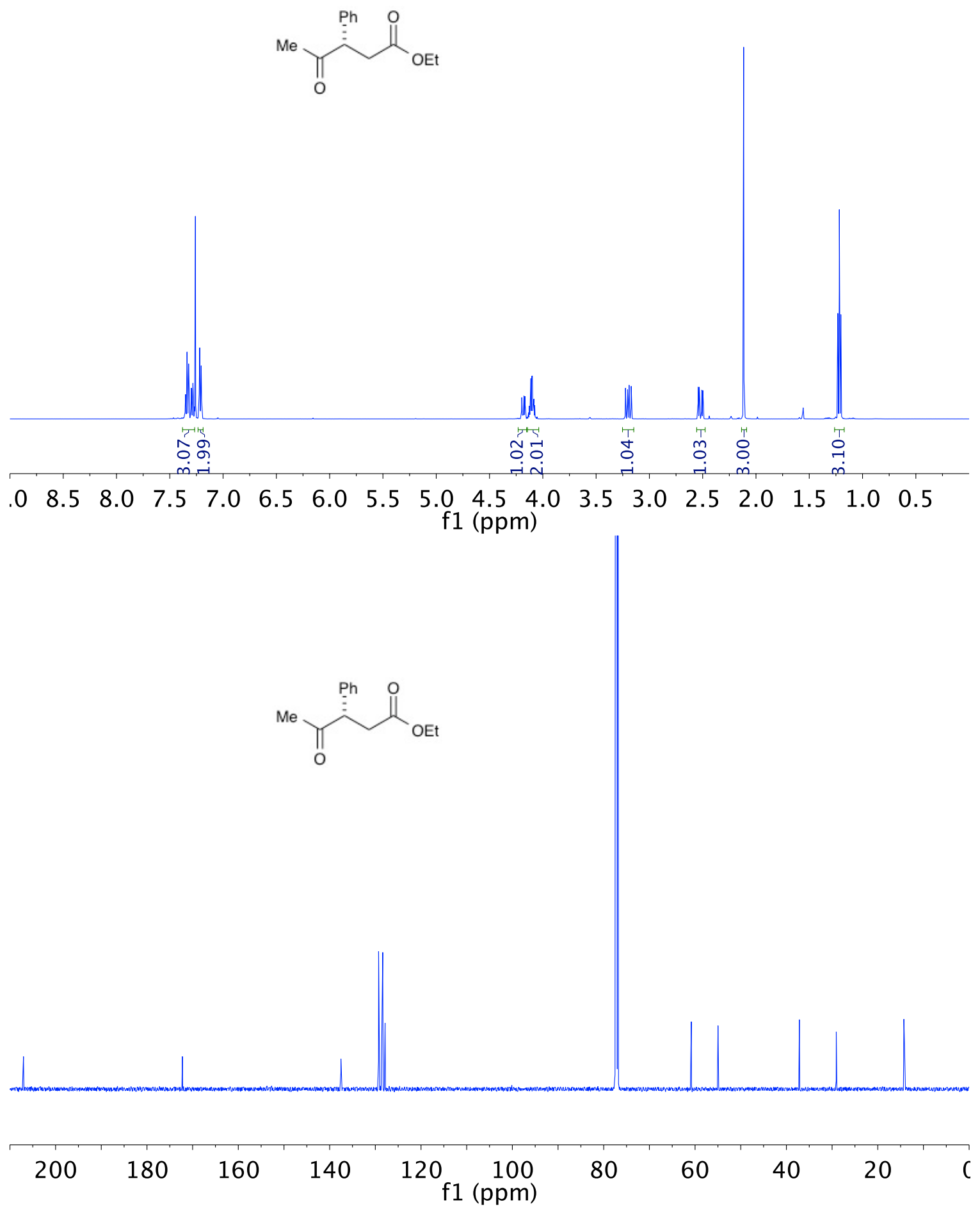



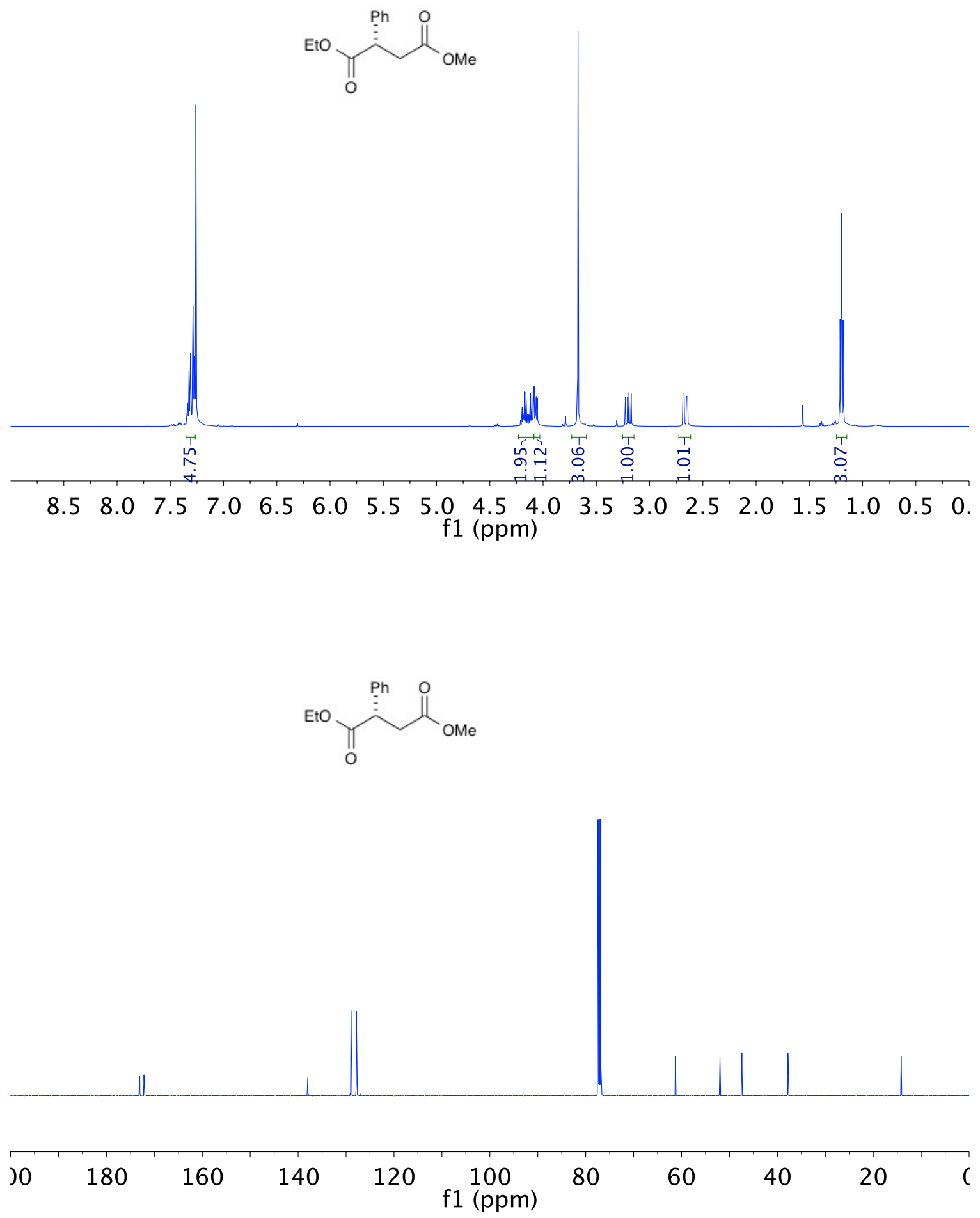

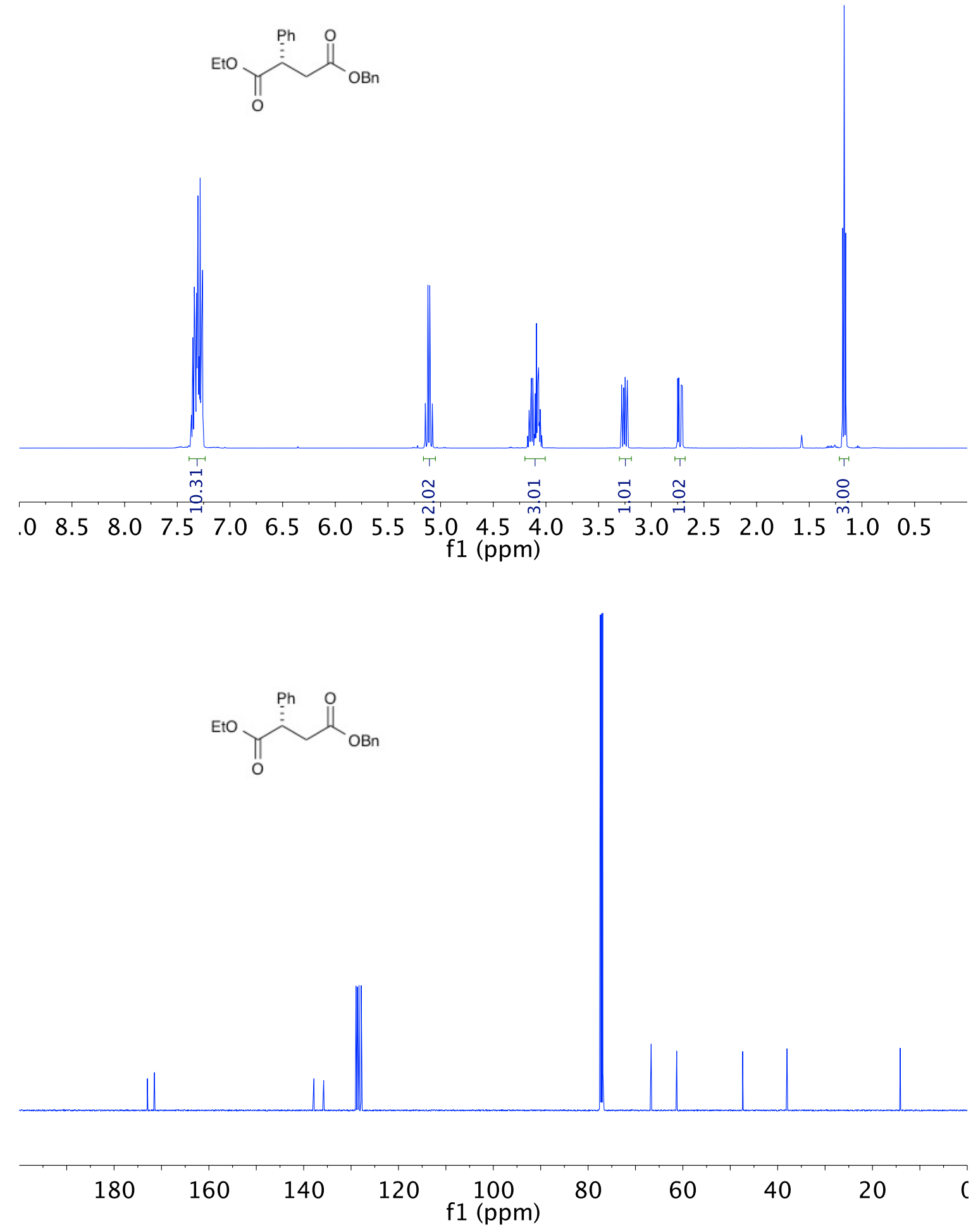

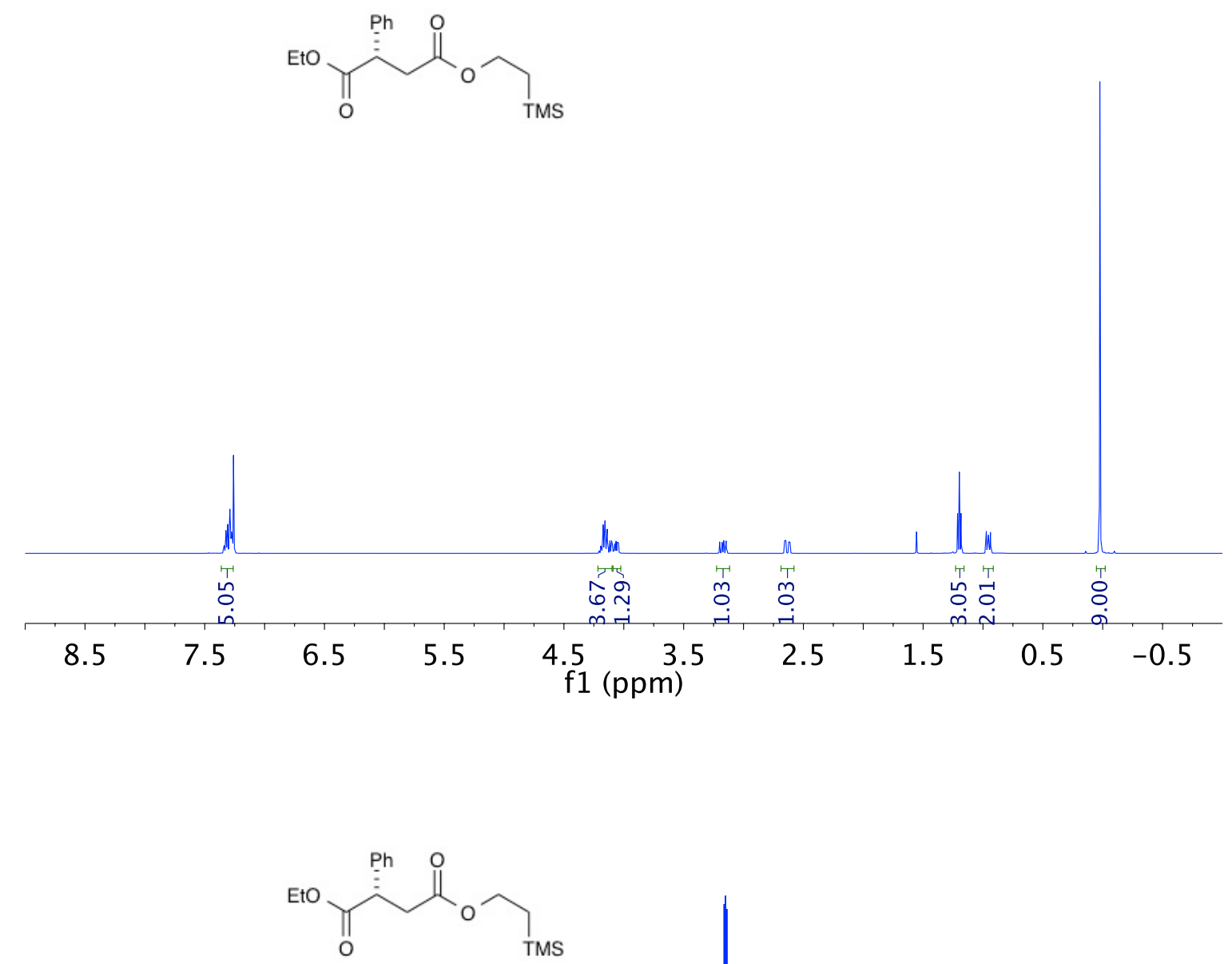


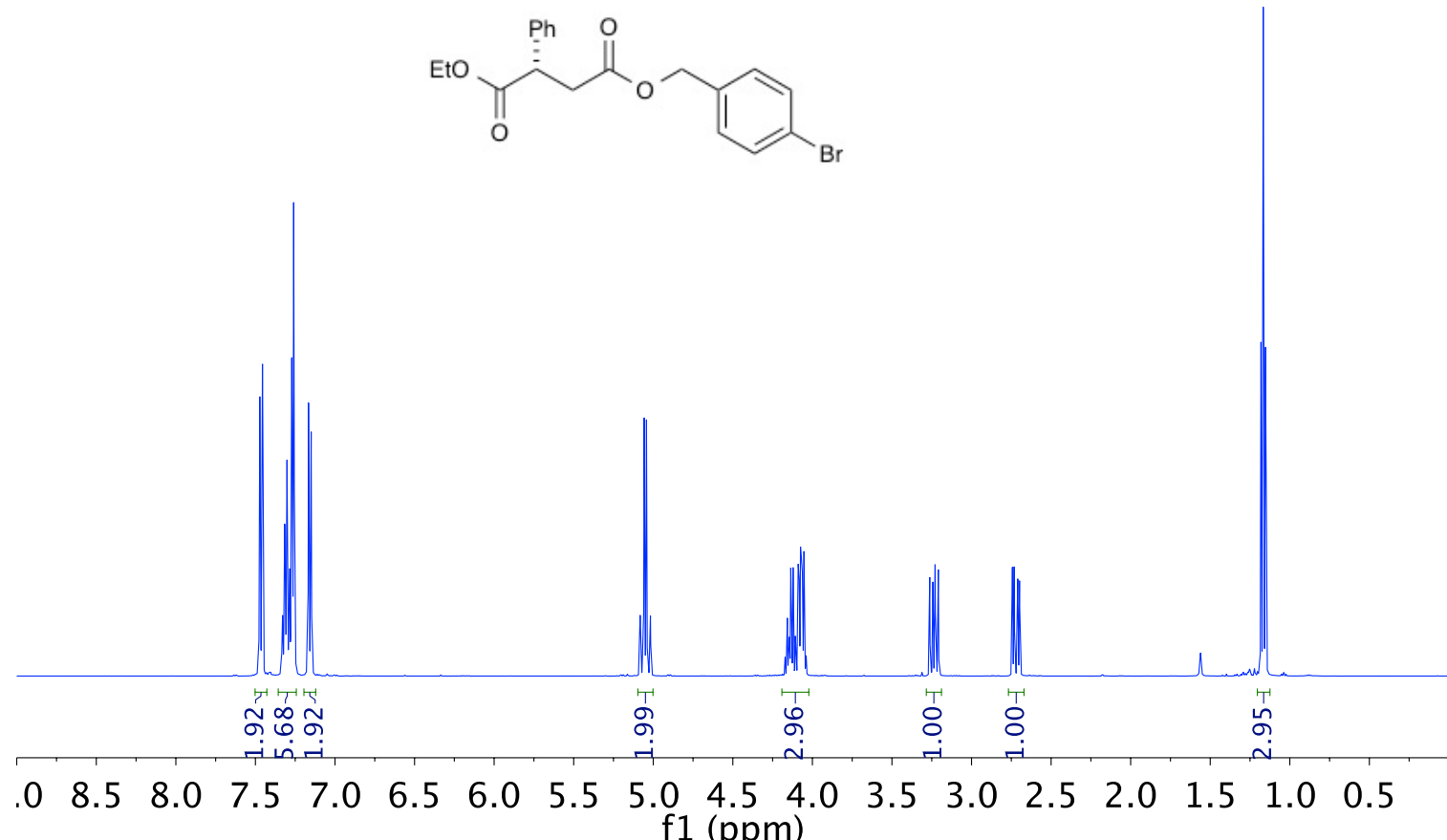
f1 (ppm)
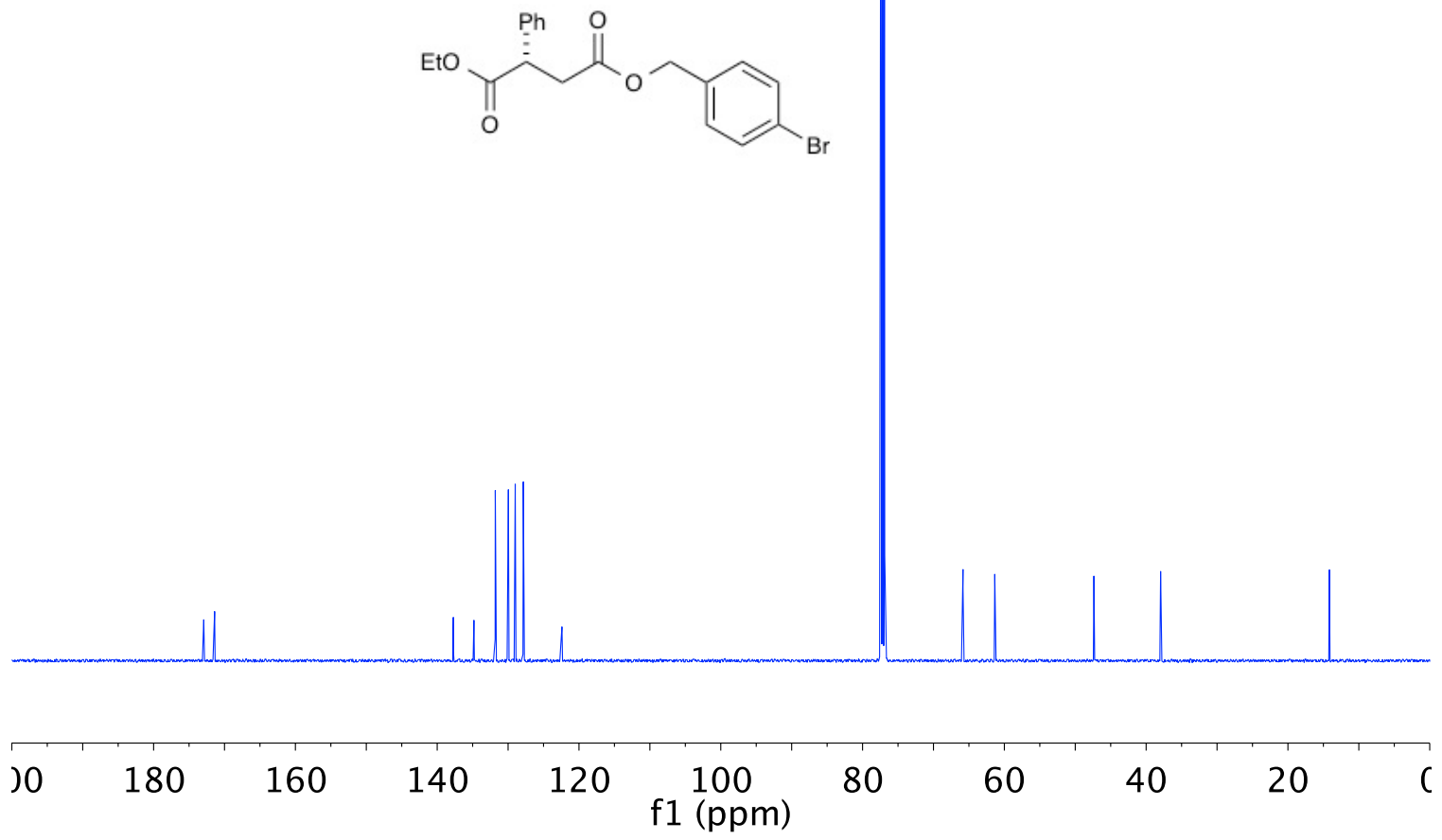


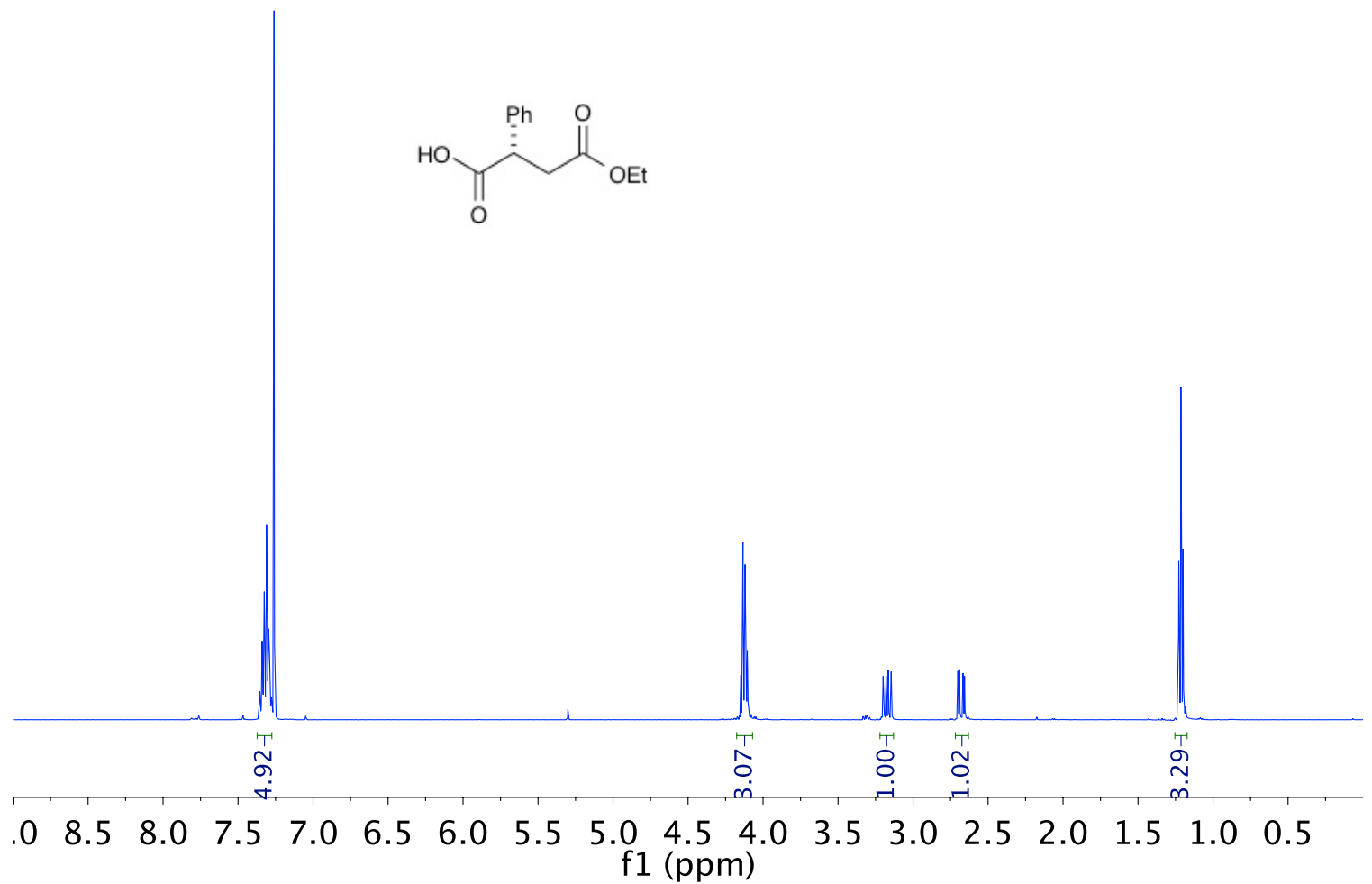<smiles>CCOC(=O)C[C@H](C(=O)O)c1ccccc1</smiles>

$30 \quad 180 \quad 160 \quad 140 \quad 120 \begin{gathered}100 \\ \mathrm{f} 1(\mathrm{ppm})\end{gathered}$




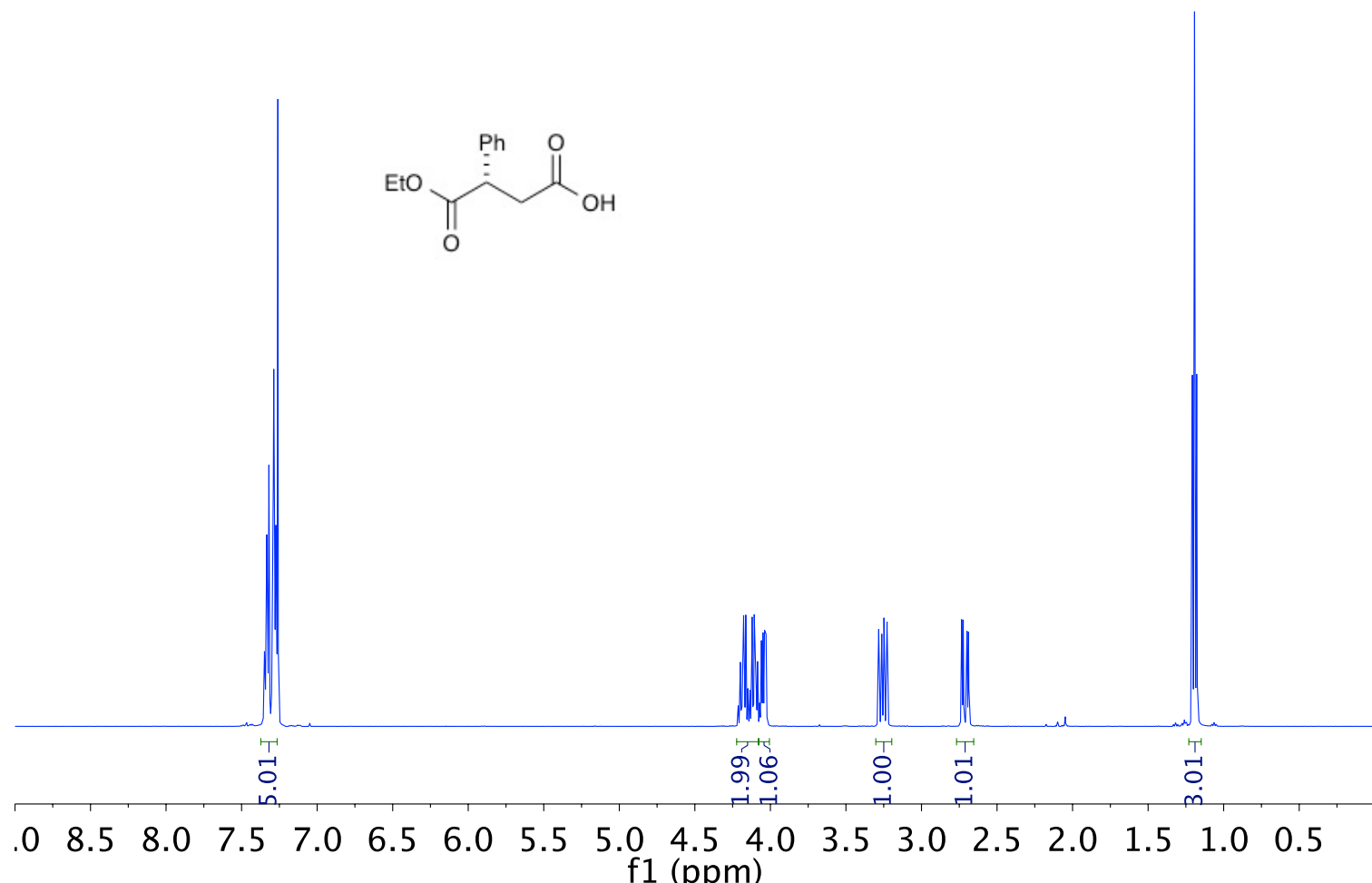
f1 (ppm)
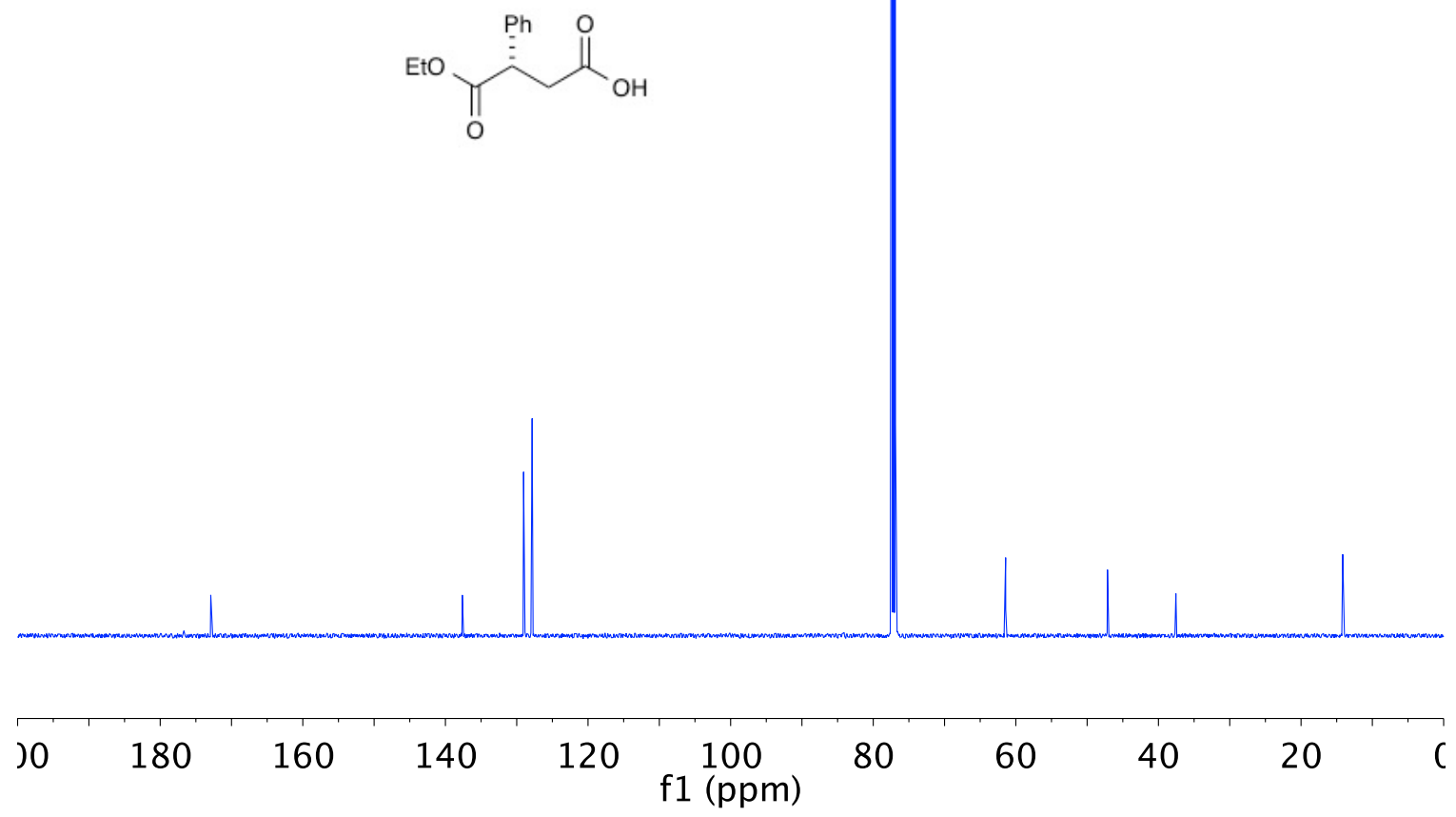


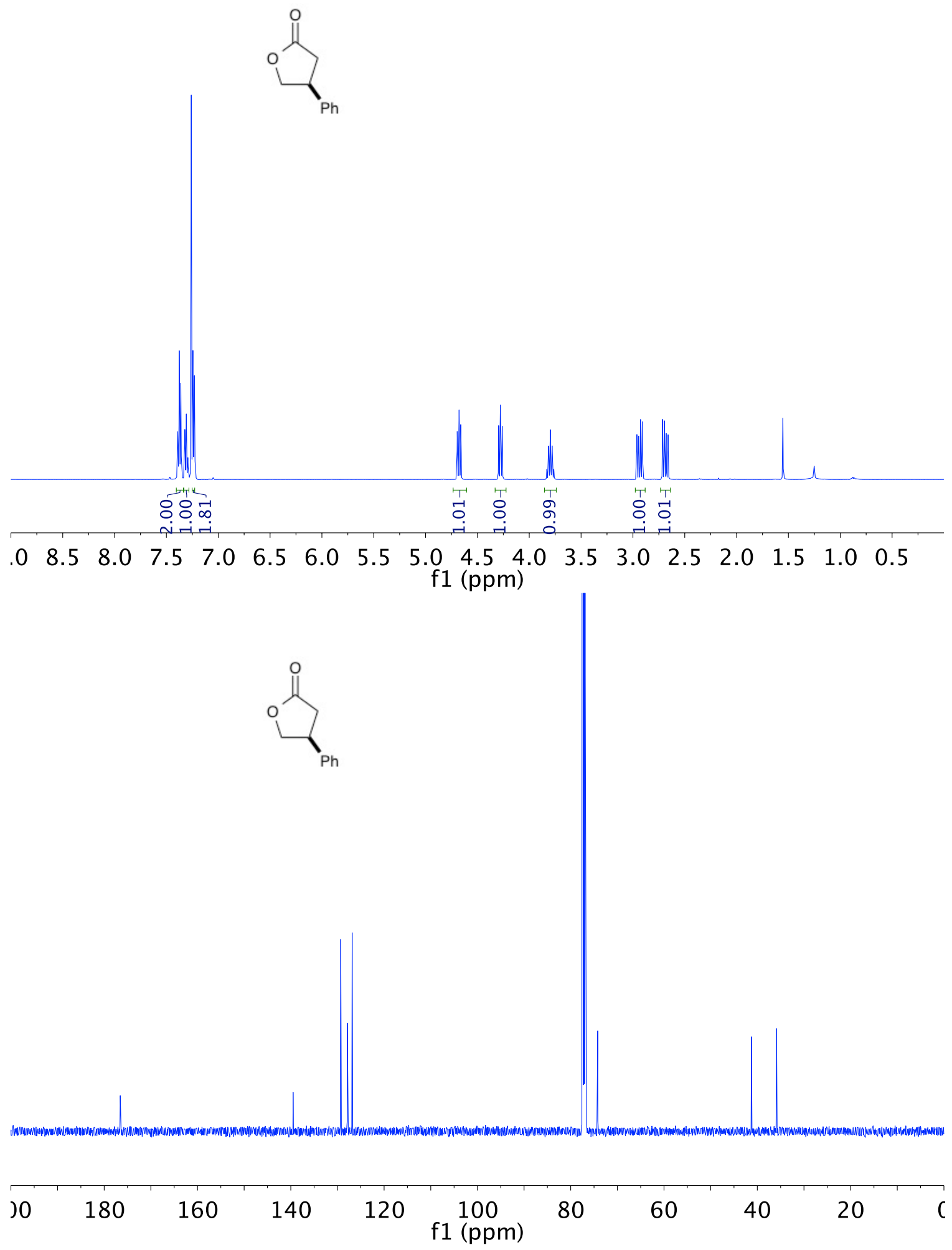



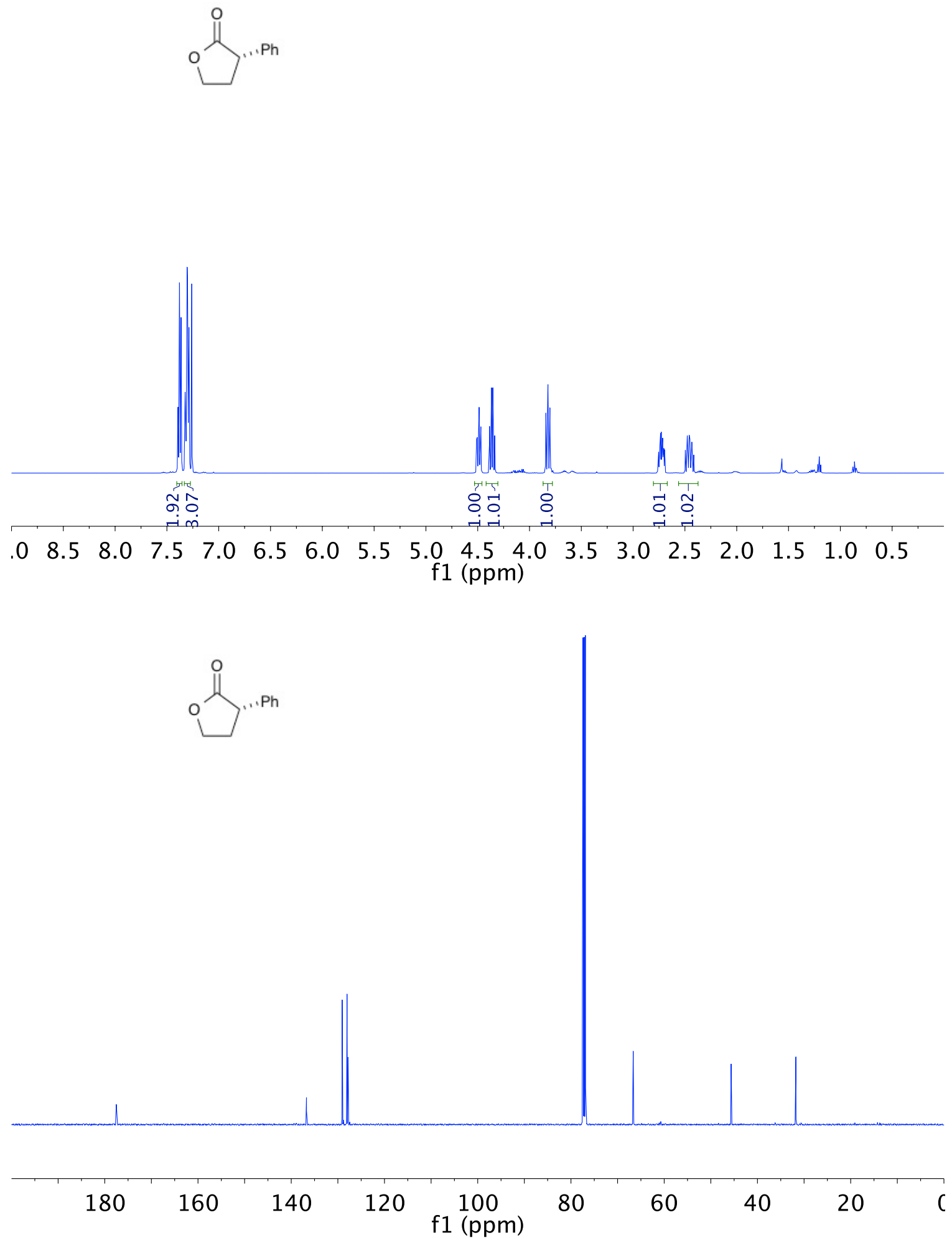


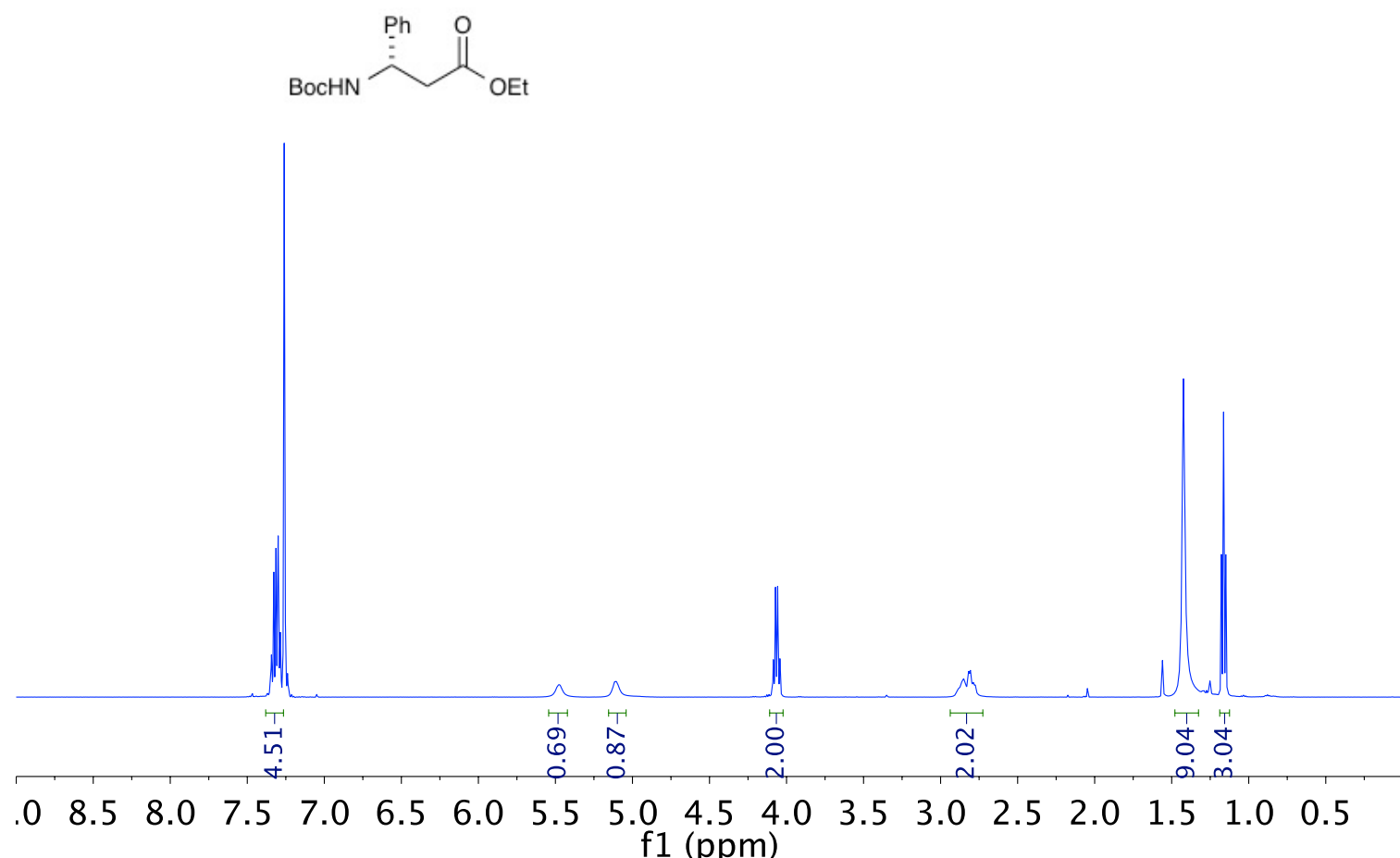
f1 (ppm)

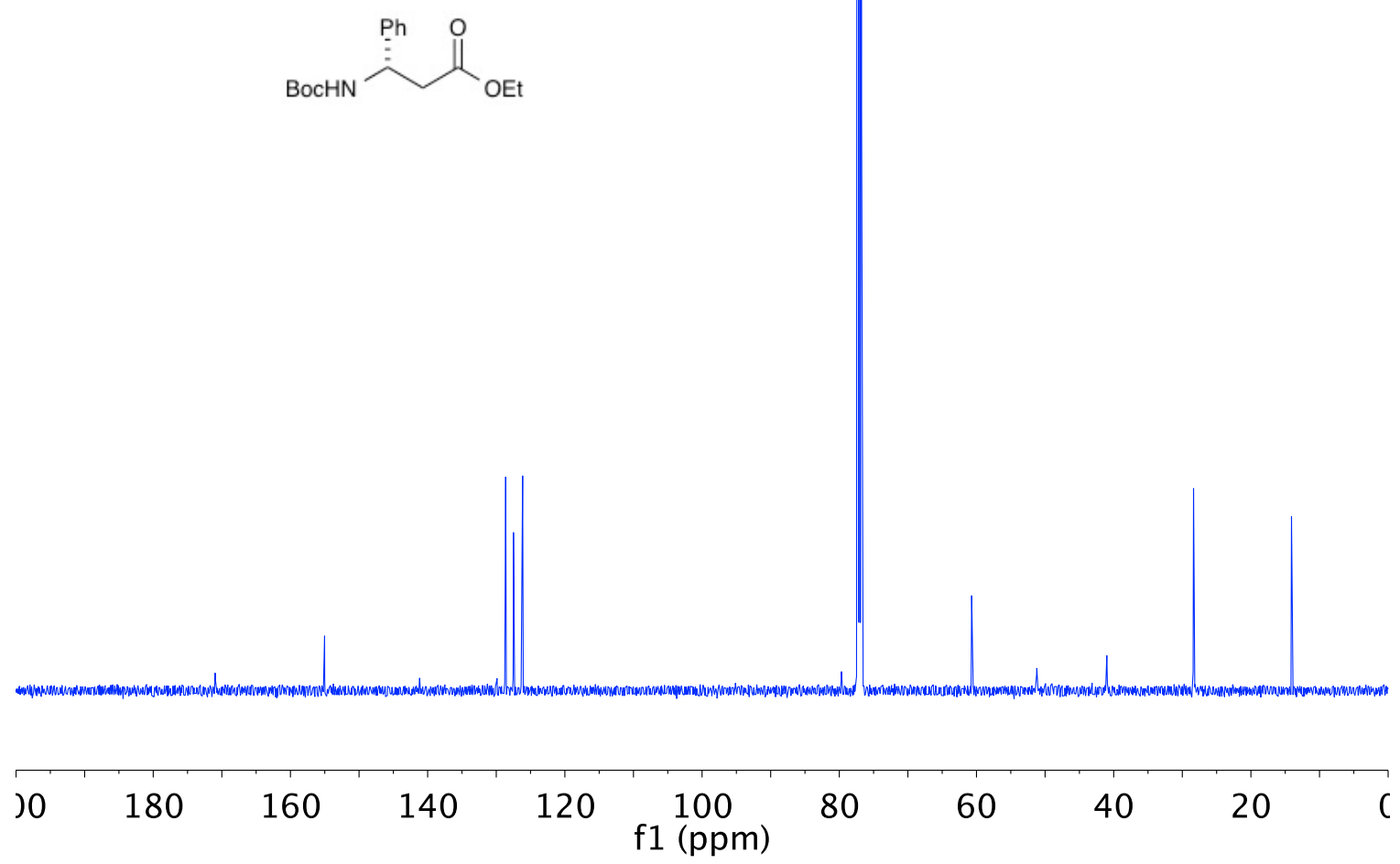



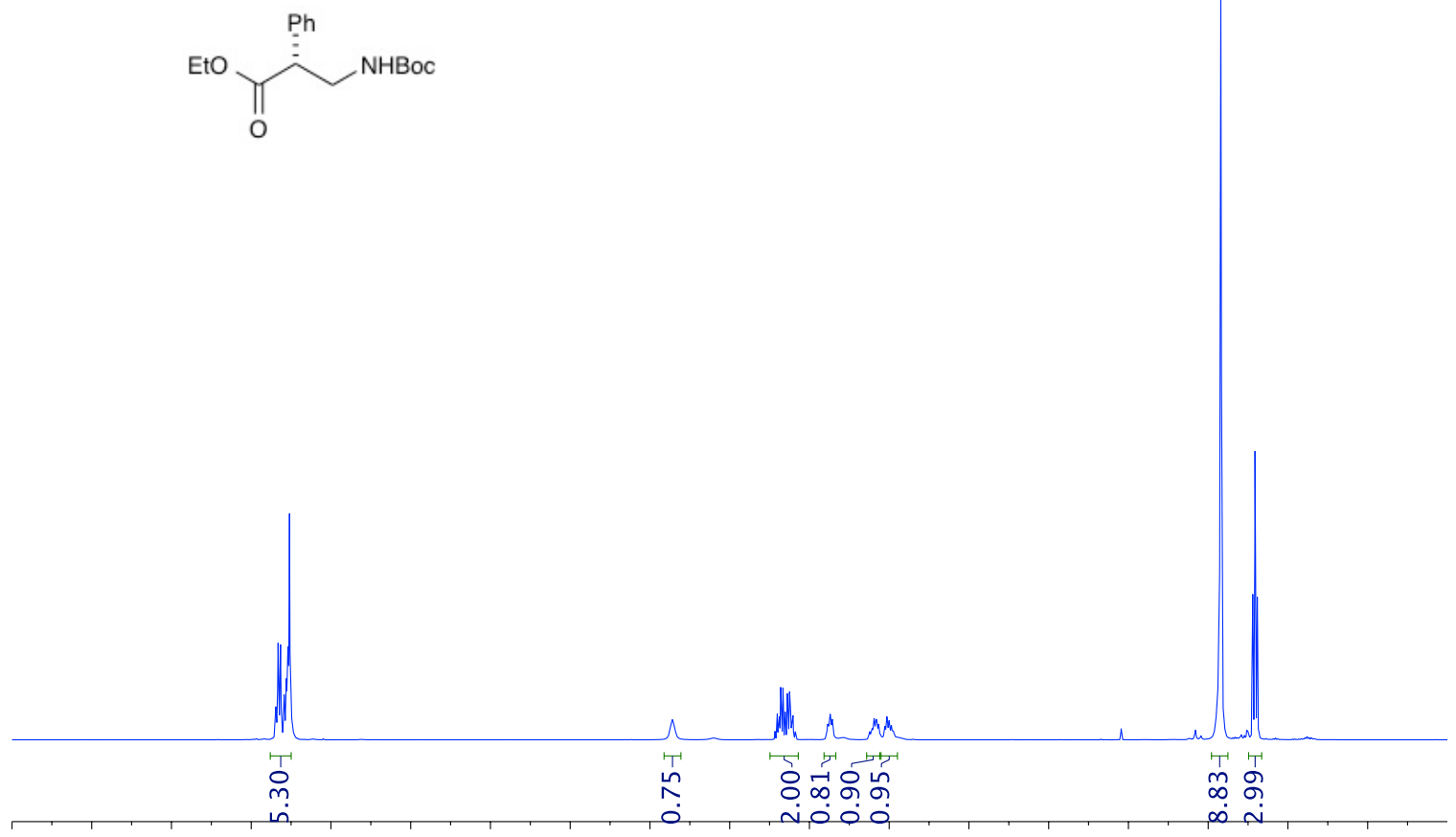

$\begin{array}{llllllllllllllllll}.0 & 8.5 & 8.0 & 7.5 & 7.0 & 6.5 & 6.0 & 5.5 & 5.0 & 4.5 & 4.0 & 3.5 & 3.0 & 2.5 & 2.0 & 1.5 & 1.0 & 0.5\end{array}$ f1 (ppm)<smiles>CCOC(=O)[C@H](CNC(=O)OCc1ccccc1)c1ccccc1</smiles>

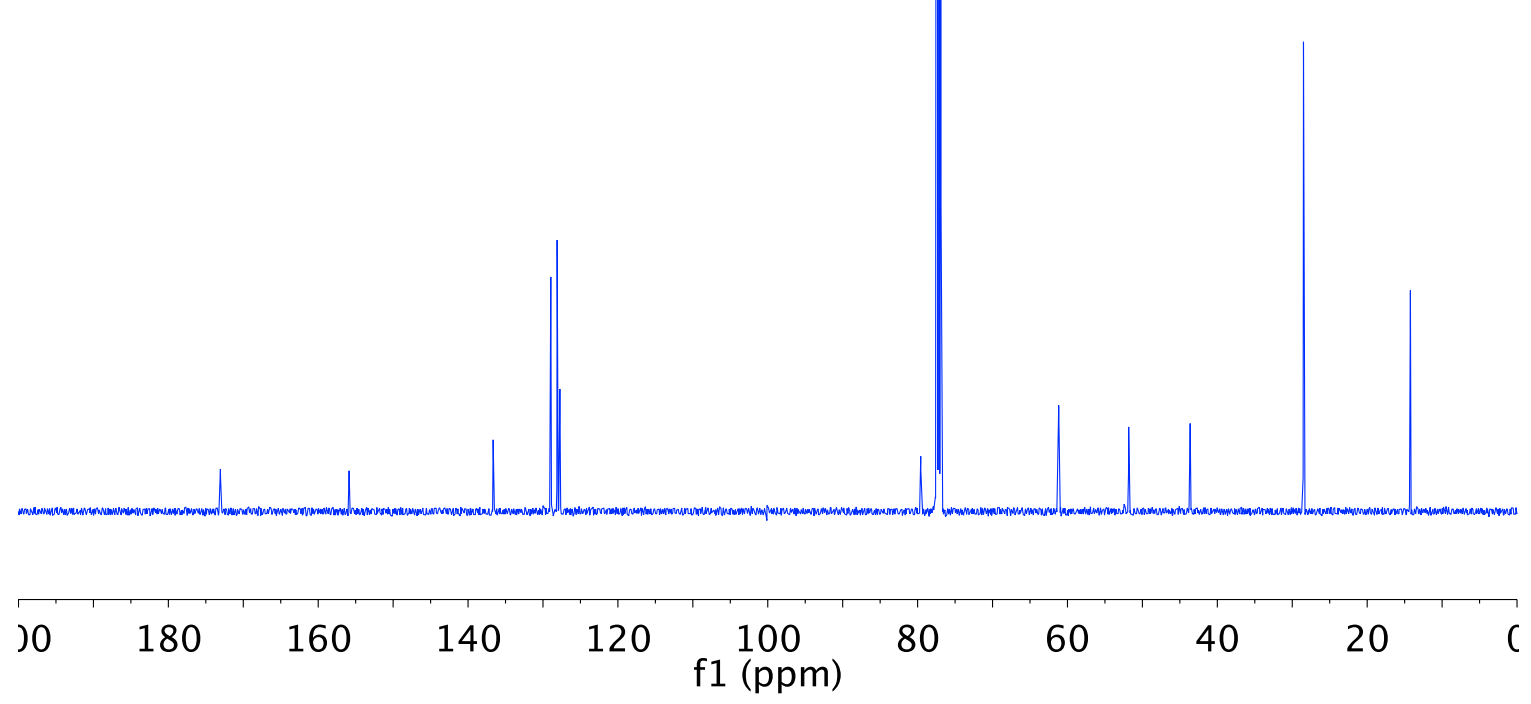




\section{HPLC Traces of Racemic and Enantioenriched Compounds}

\section{Racemic 2}

/Users/michael...17-86-PH-RAC.D/ Injection 1 DAD D, Sig=230,16 Ref=360,100 Chromatogram

\begin{tabular}{|c|l|}
\hline RT & Total Area $\%$ \\
\hline 31.965 & 49.53 \\
\hline 35.971 & 50.47 \\
\hline
\end{tabular}

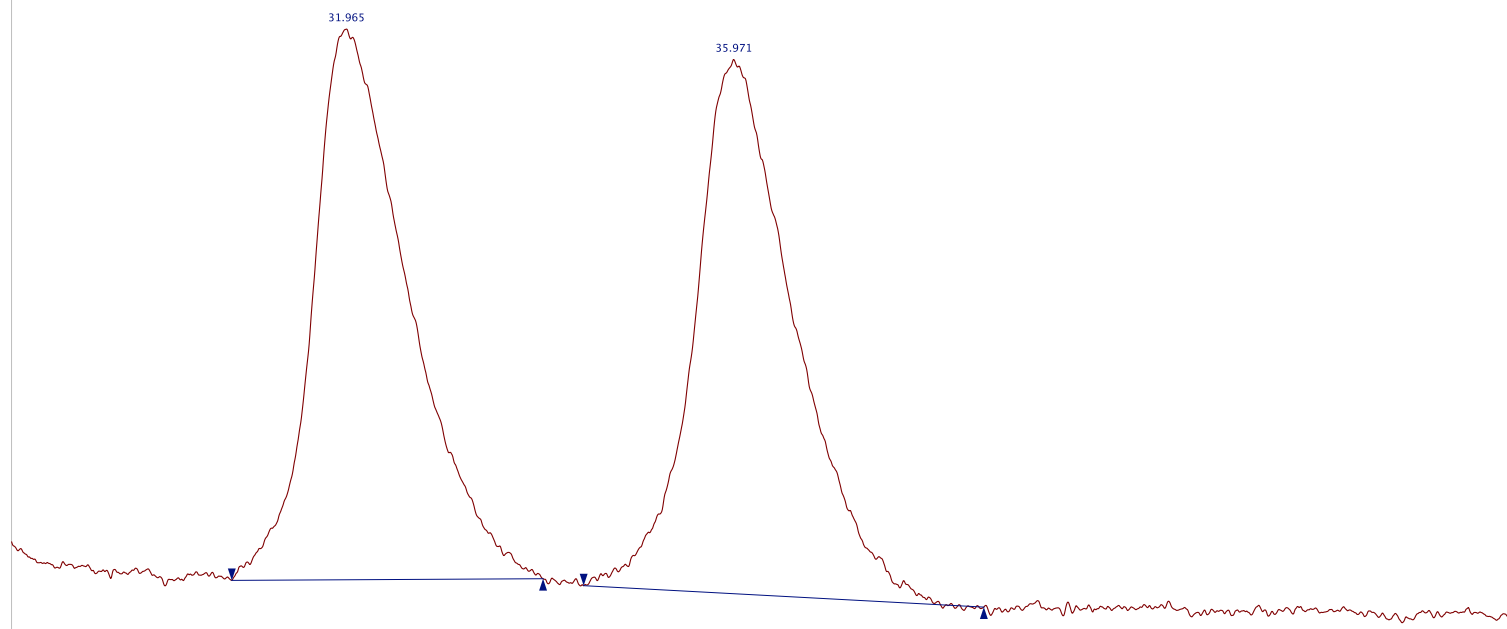

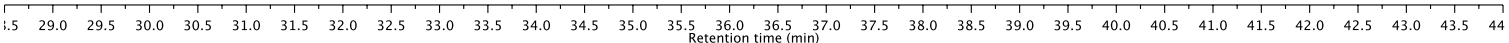

Enantioenriched 2

\begin{tabular}{|c|l|}
\hline RT & Total Area $\%$ \\
\hline 31.728 & 4.39 \\
\hline 34.034 & 95.61 \\
\hline
\end{tabular}

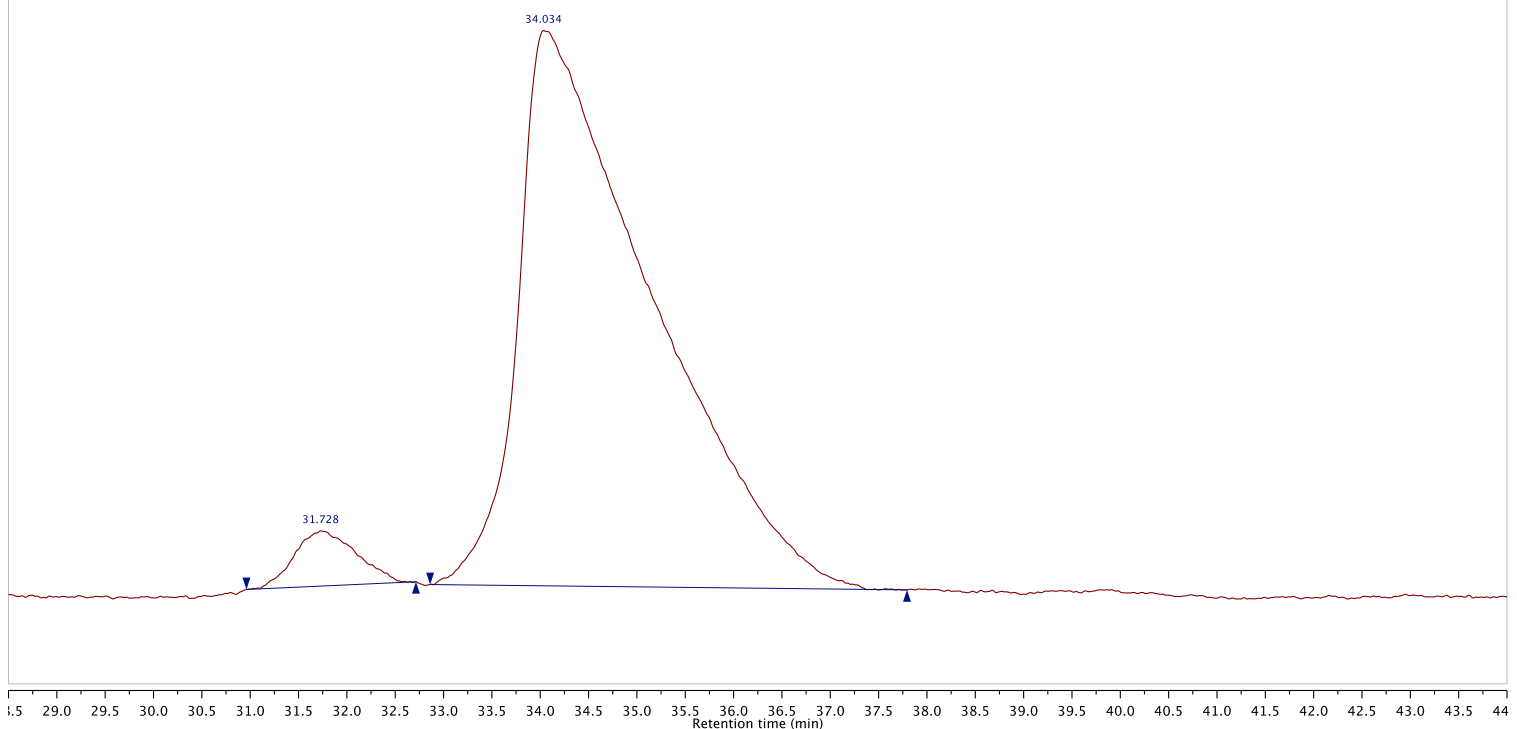




\section{Racemic 3}

/Users/michael...93-2NAPH-RAC.D/ Injection 1 DAD1D, Sig=230,4 Ref=off Chromatogram

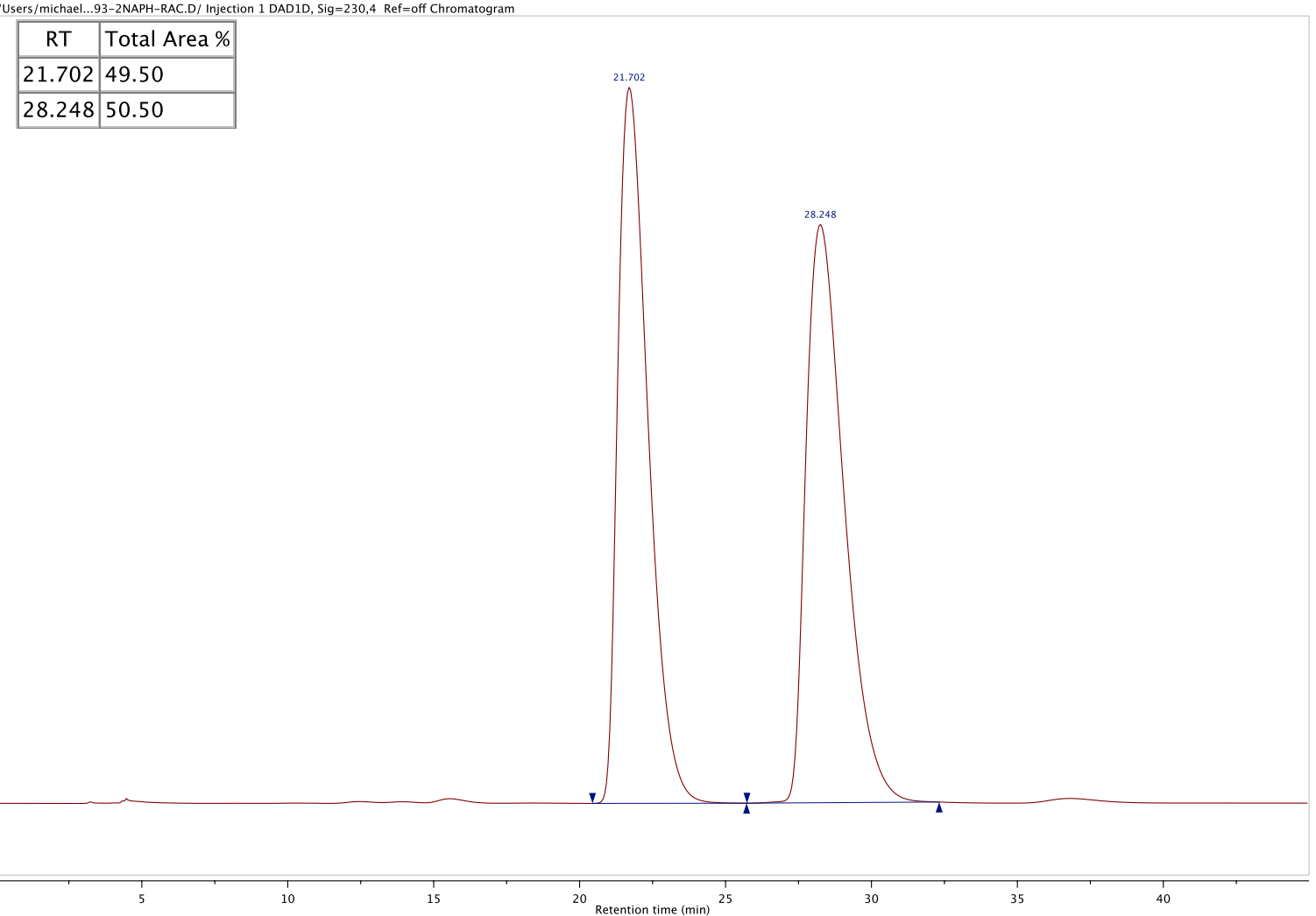

\section{Enantioenriched 3}

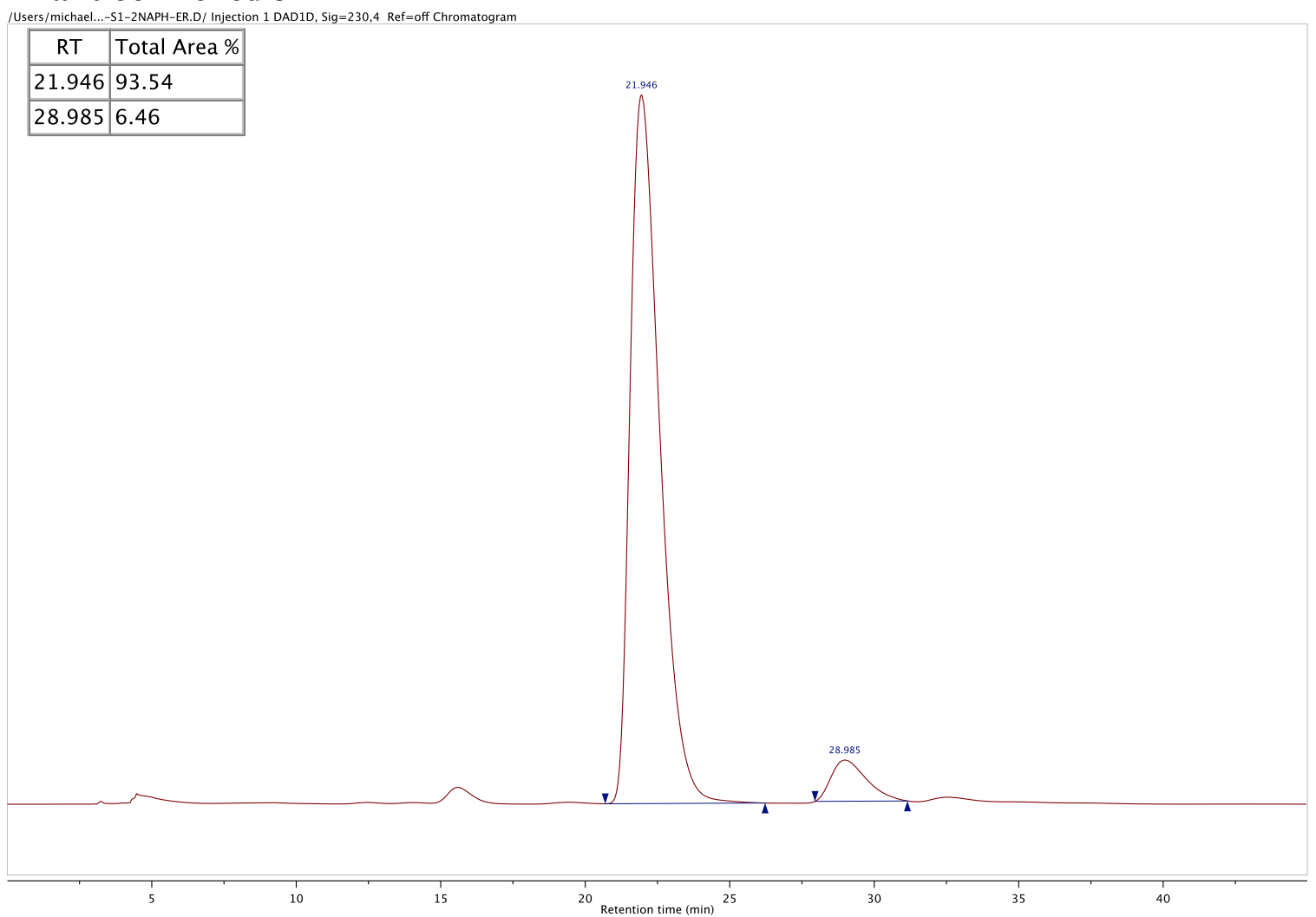




\section{Racemic 4}

IUsers/michael...5-28-4ME-RAC.D/ Injection 1 DAD1C, Sig=210,4 Ref=off Chromatogram

\begin{tabular}{|c|l|l|}
\hline RT & Total Area \% \\
\hline
\end{tabular}

6.98149 .85

\begin{tabular}{|l|l|l|l|}
\hline 8.561 & 50.15 \\
\hline
\end{tabular}

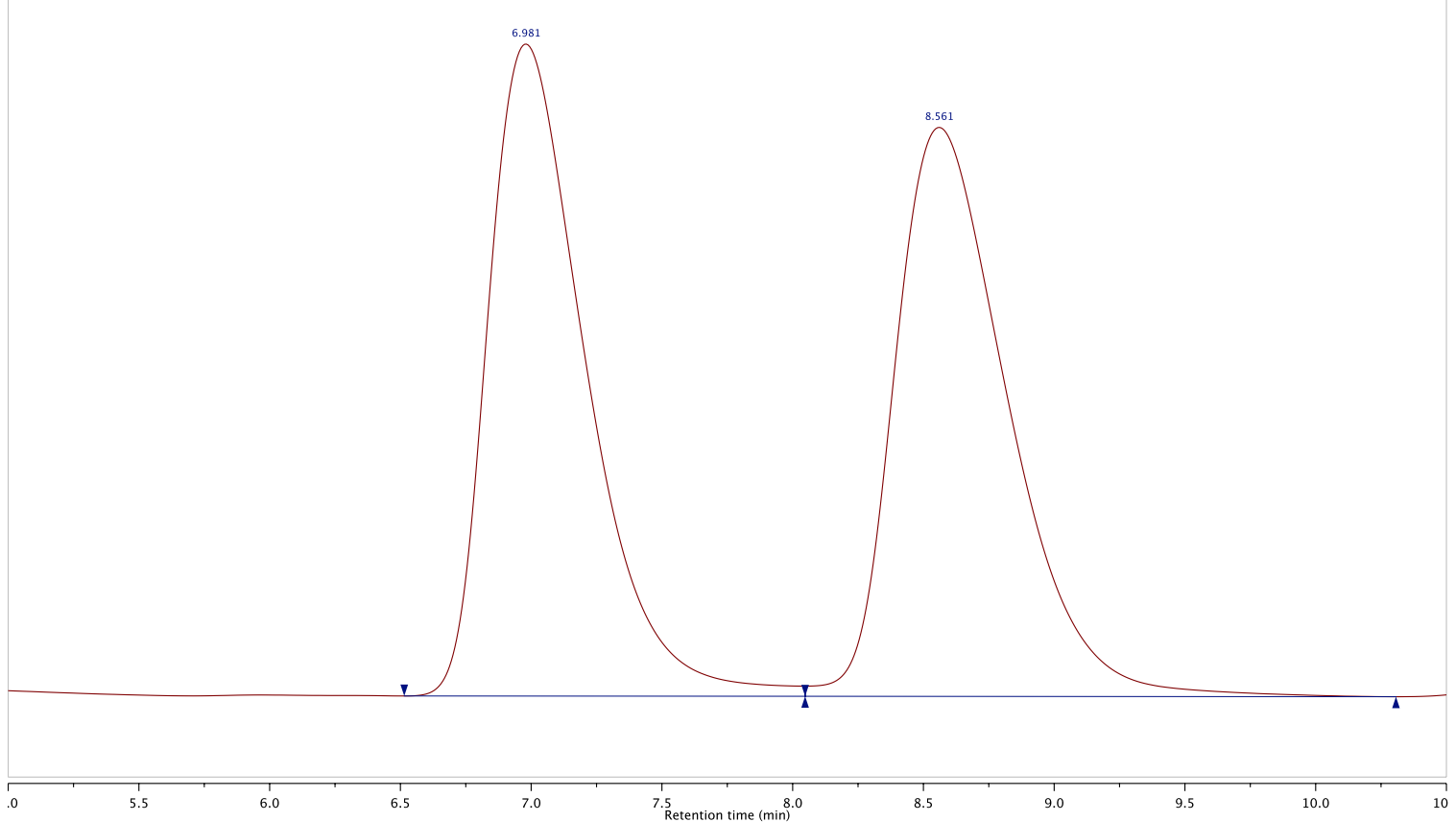

Enantioenriched 4

/Users/michael...07-S1-4ME-ER.D/ Injection 1 DAD1C, Sig=210,4 Ref=off Chromatogram RT Total Area \%

$6.962 \quad 91.14$

8.5888 .86

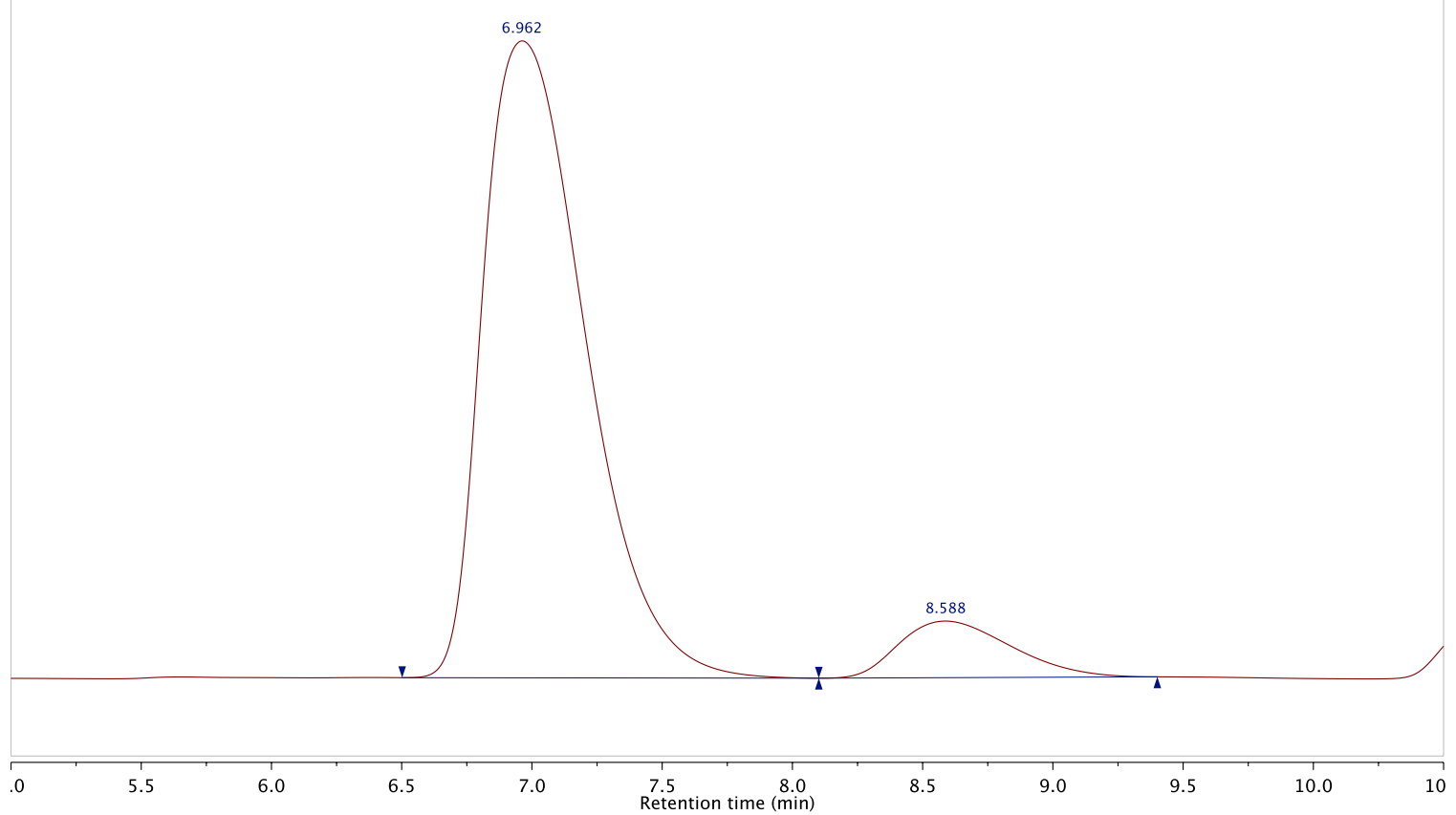




\section{Racemic 5}

/Users/michael...5-26-3ME-RAC.D/ Injection 1 DAD1C, Sig=210,4 Ref=off Chromatogram

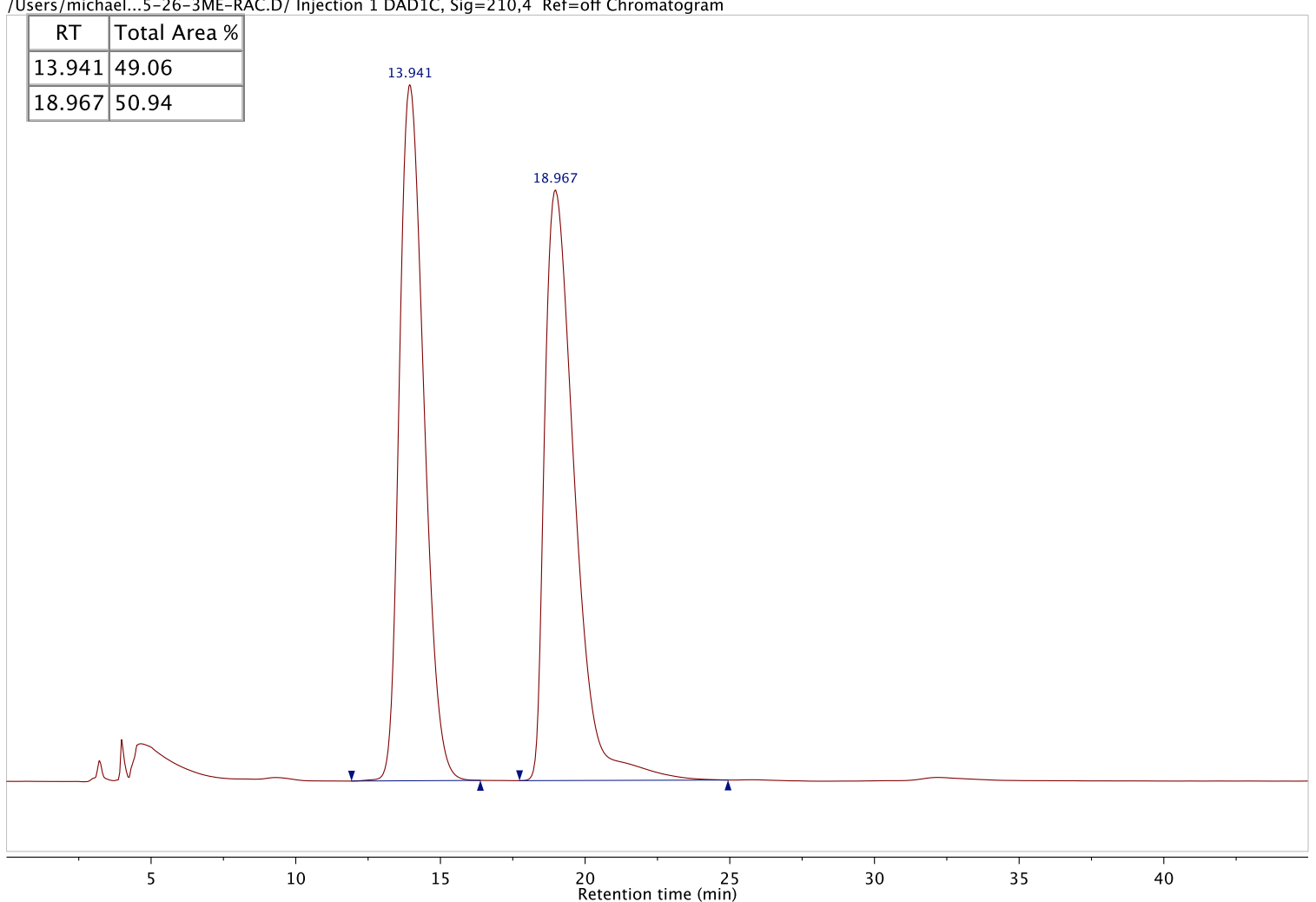

\section{Enantioenriched 5}

/Users/michael...08-S1-3ME-ER.D/ Injection 1 DAD1C, Sig=210,4 Ref=off Chromatogram

\begin{tabular}{|c|l|}
\hline RT & Total Area \% \\
\hline 13.887 & 95.90 \\
\hline 19.253 & 4.10 \\
\hline
\end{tabular}

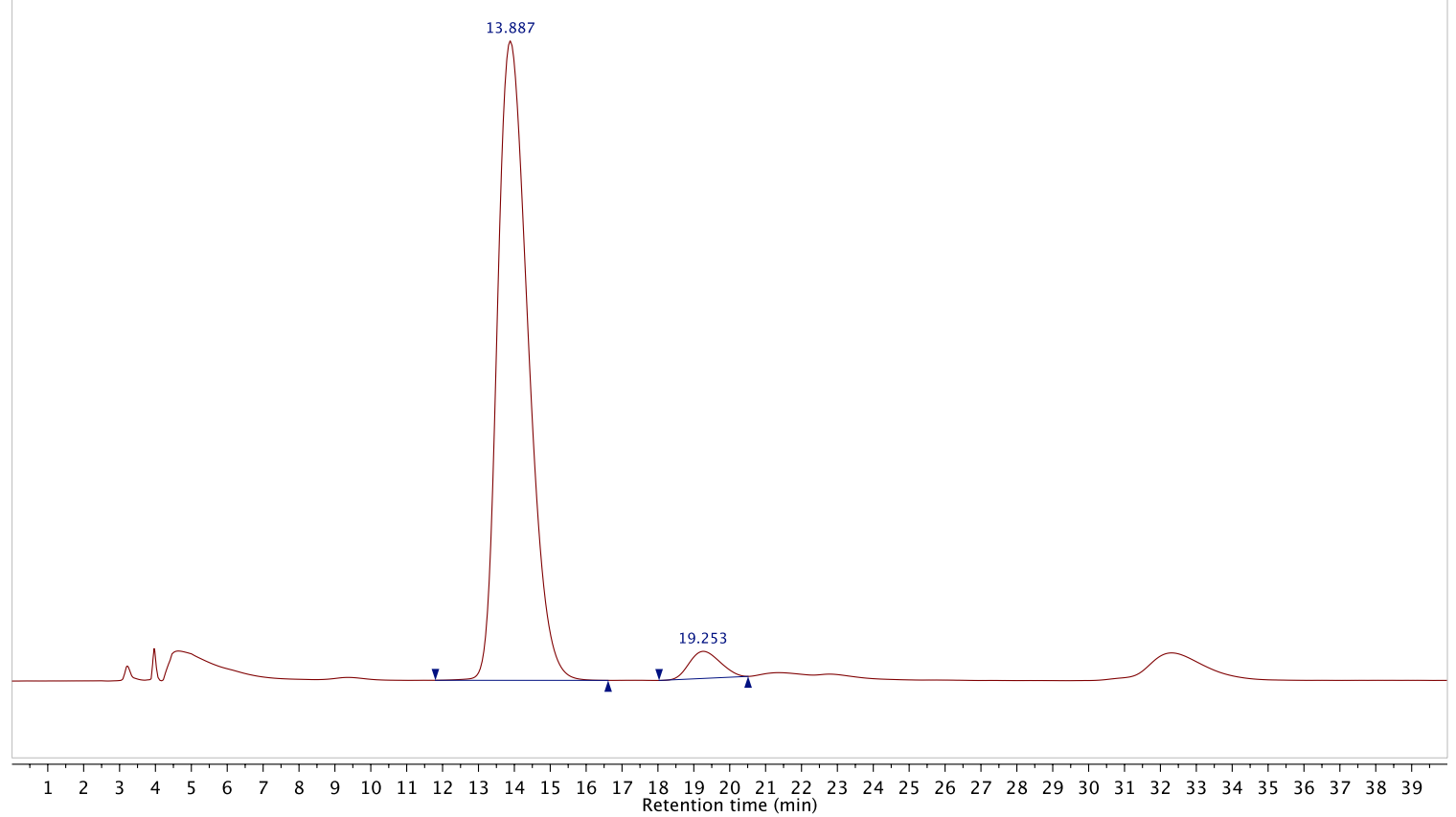




\section{Racemic 6}

/Users/michael...-94-4OME-RAC.D/ Injection 1 DAD1C, Sig=210,4 Ref=off Chromatogram

\begin{tabular}{|c|l|}
\hline RT & Total Area \% \\
\hline 9.425 & 50.17 \\
\hline 10.278 & 49.83 \\
\hline
\end{tabular}

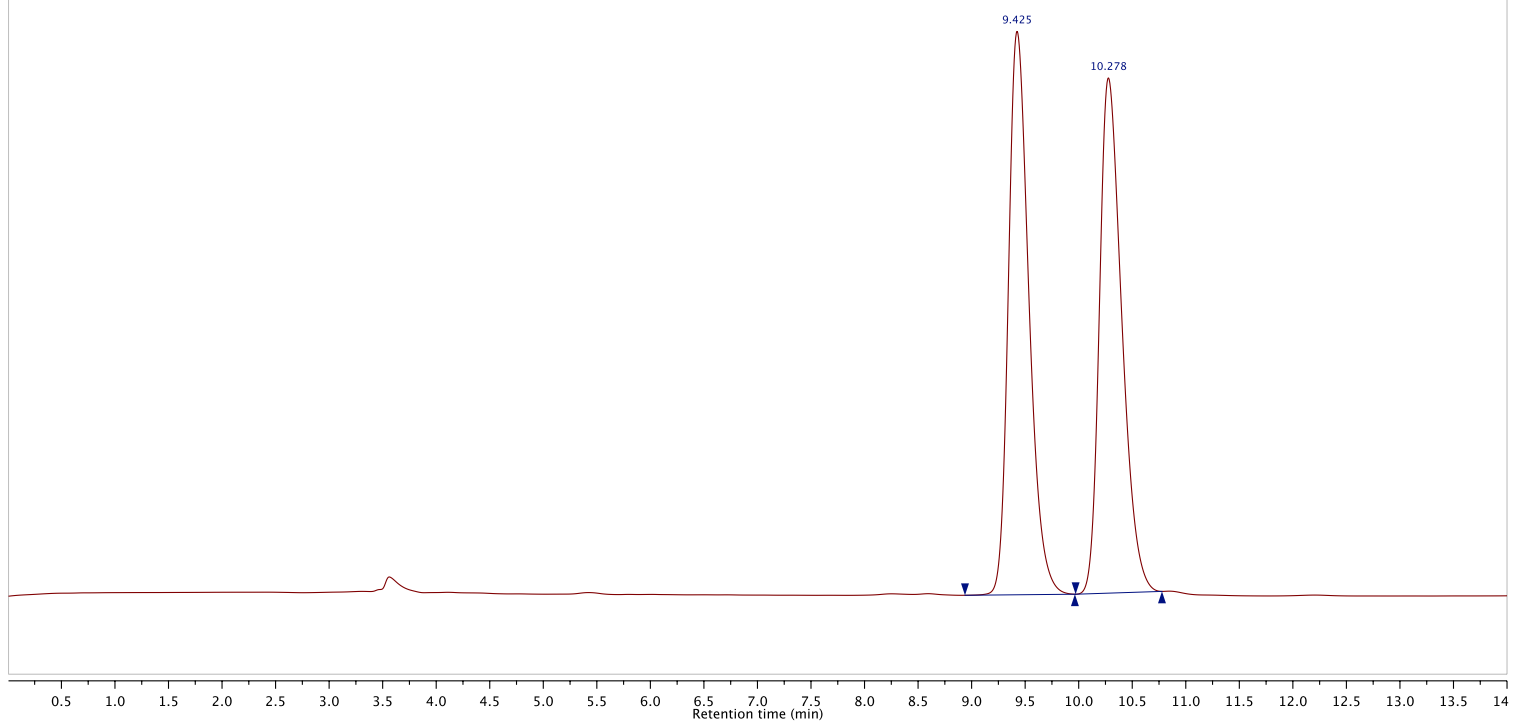

\section{Enantioenriched 6}

/Users/michael...5-S1-4OME-ER.D/ Injection 1 DAD1C, Sig=210,4 Ref=off Chromatogram

\begin{tabular}{|l|l|}
\hline \multicolumn{1}{|c|}{ RT } & Total Area \% \\
\hline 9.475 & 7.66 \\
\hline 10.235 & 92.34 \\
\hline
\end{tabular}

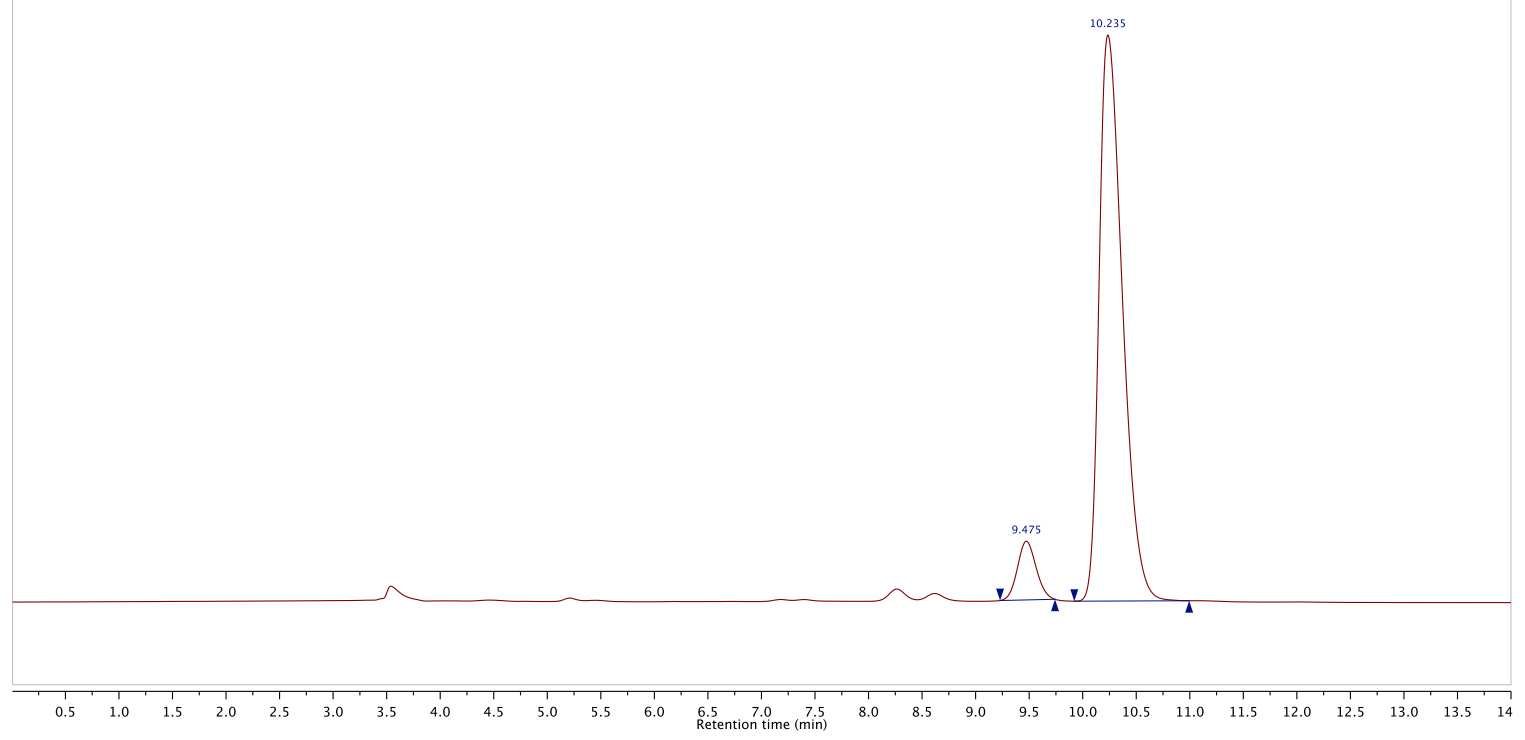




\section{Racemic 7}

IUsers/michael...14-34OME-RAC.D/ Injection 1 DAD1C, Sig=210,4 Ref=off Chromatogram

\begin{tabular}{|c|l|}
\hline RT & Total Area \% \\
\hline 24.503 & 48.46 \\
\hline 28.569 & 51.54 \\
\hline
\end{tabular}

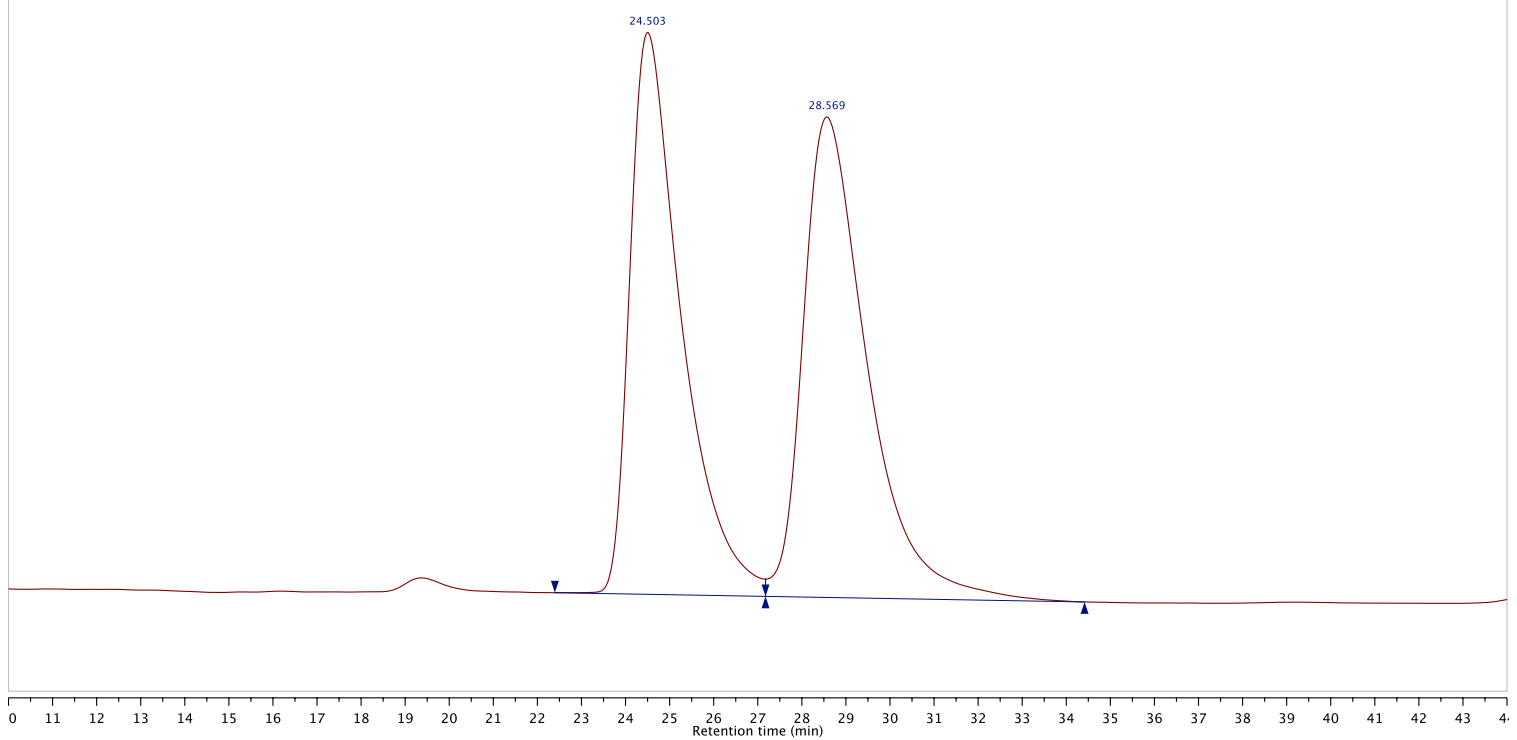

\section{Enantioenriched 7}

/USers/michael...-S1-340ME-ER.D/ Injection 1 DAD1C, sig=210,4 Ref=off Chromatogram

\begin{tabular}{|c|l|}
\hline RT & Total Area \% \\
\hline 24.241 & 7.16 \\
\hline 28.340 & 92.84 \\
\hline
\end{tabular}

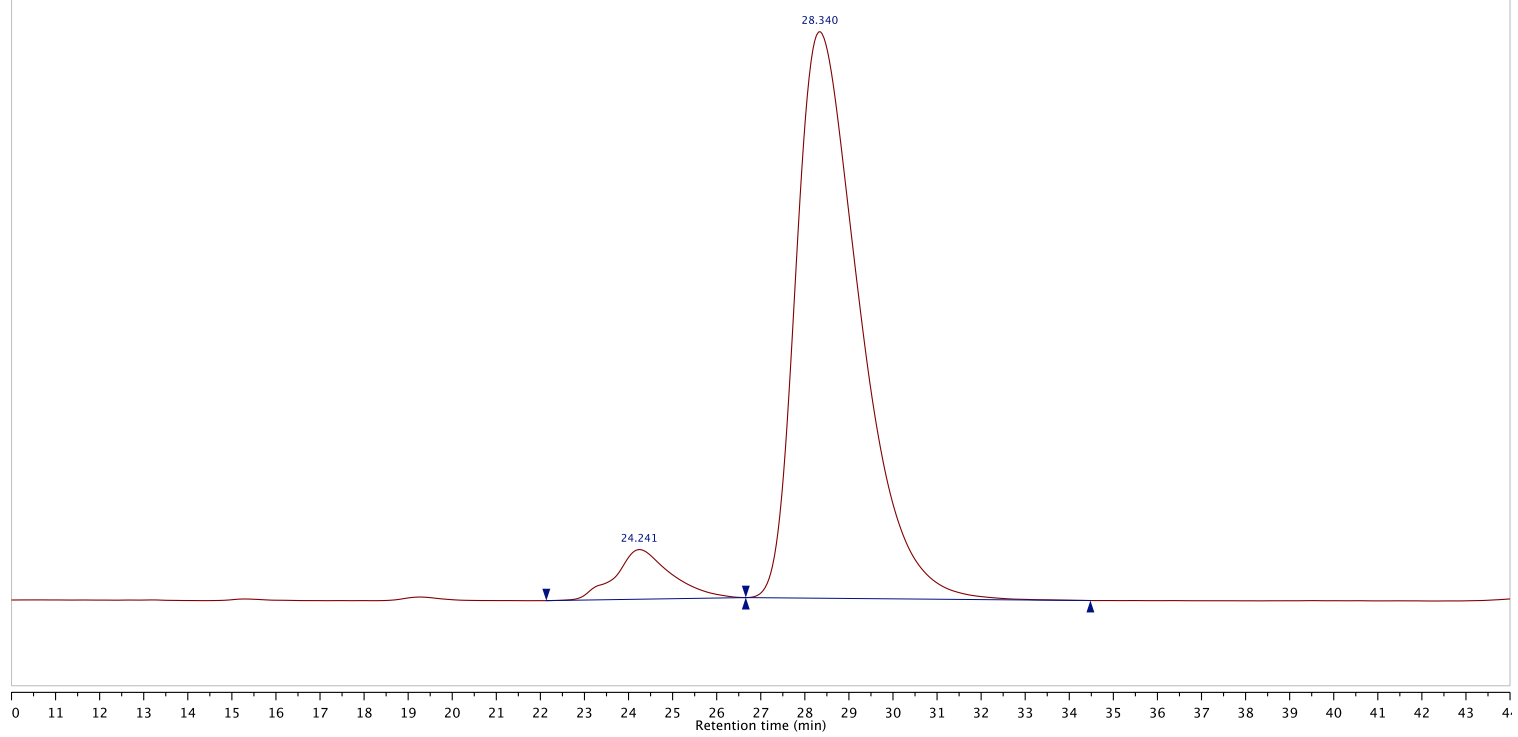




\section{Racemic 8}

/Users/michael...17-84-4F-RAC.D/ Injection 1 DADIC, Sig=210,4 Ref=off Chromatogram

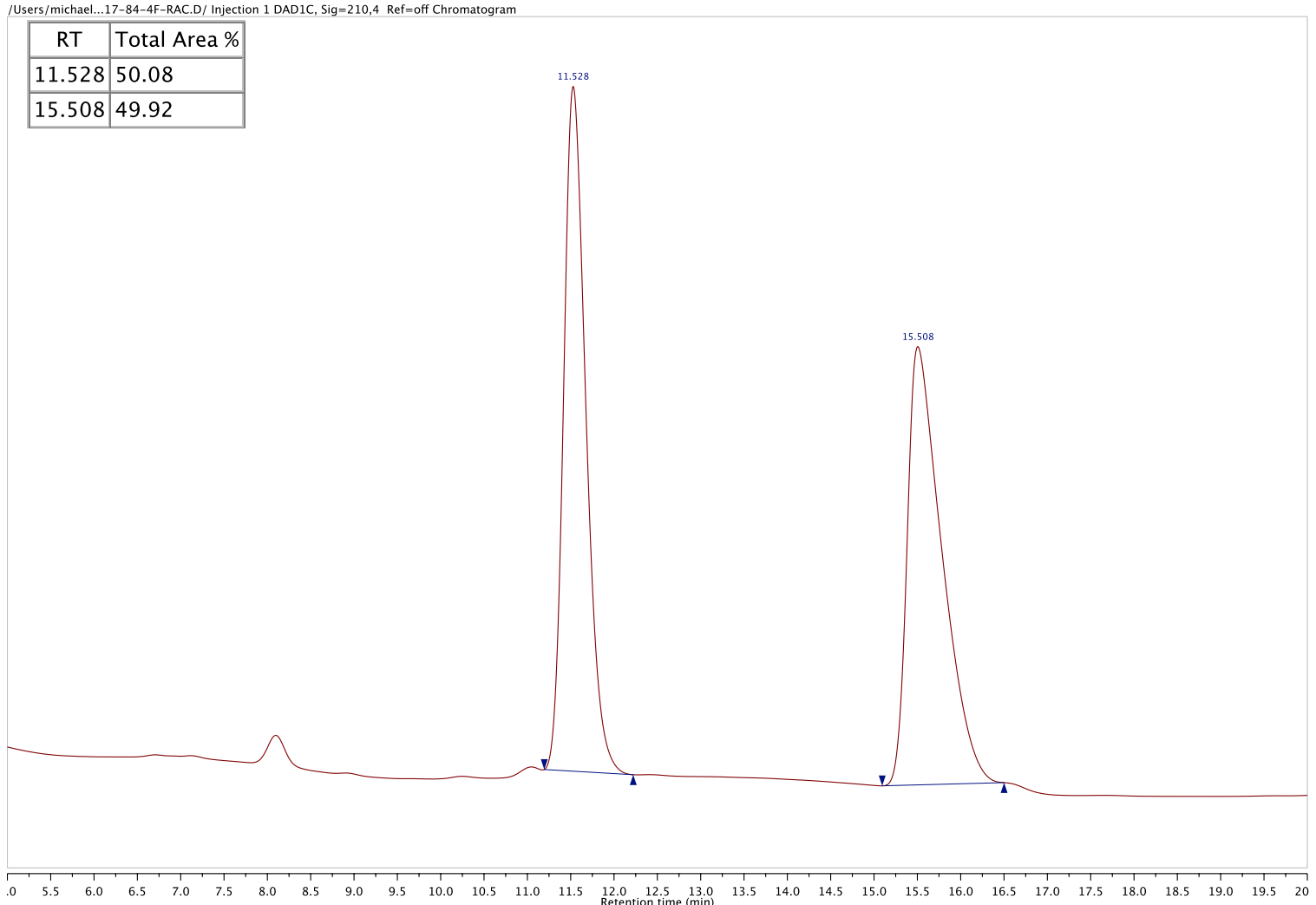

\section{Enantioenriched 8}

/Users/michael...-16-S1-4F-ER.D/ Injection 1 DAD1C, Sig=210,4 Ref=off Chromatogram

\begin{tabular}{|c|l|}
\hline RT & Total Area \% \\
\hline 16.557 & 92.58 \\
\hline 12.104 & 7.42 \\
\hline
\end{tabular}
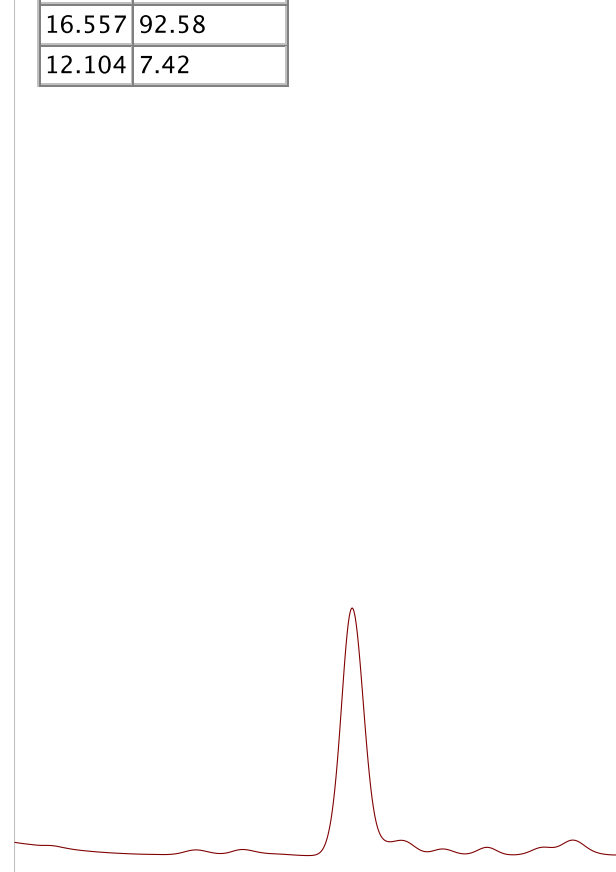

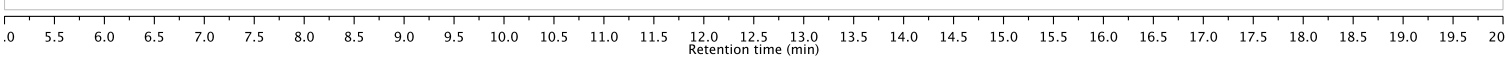




\section{Racemic 9}

/Users/michael...-PLUG-3F-RAC.D/ Injection 1 DAD1C, Sig=210,4 Ref=off Chromatogram

\begin{tabular}{|c|l|}
\hline RT & Total Area \% \\
\hline 8.155 & 50.08 \\
\hline 17.546 & 49.92 \\
\hline
\end{tabular}

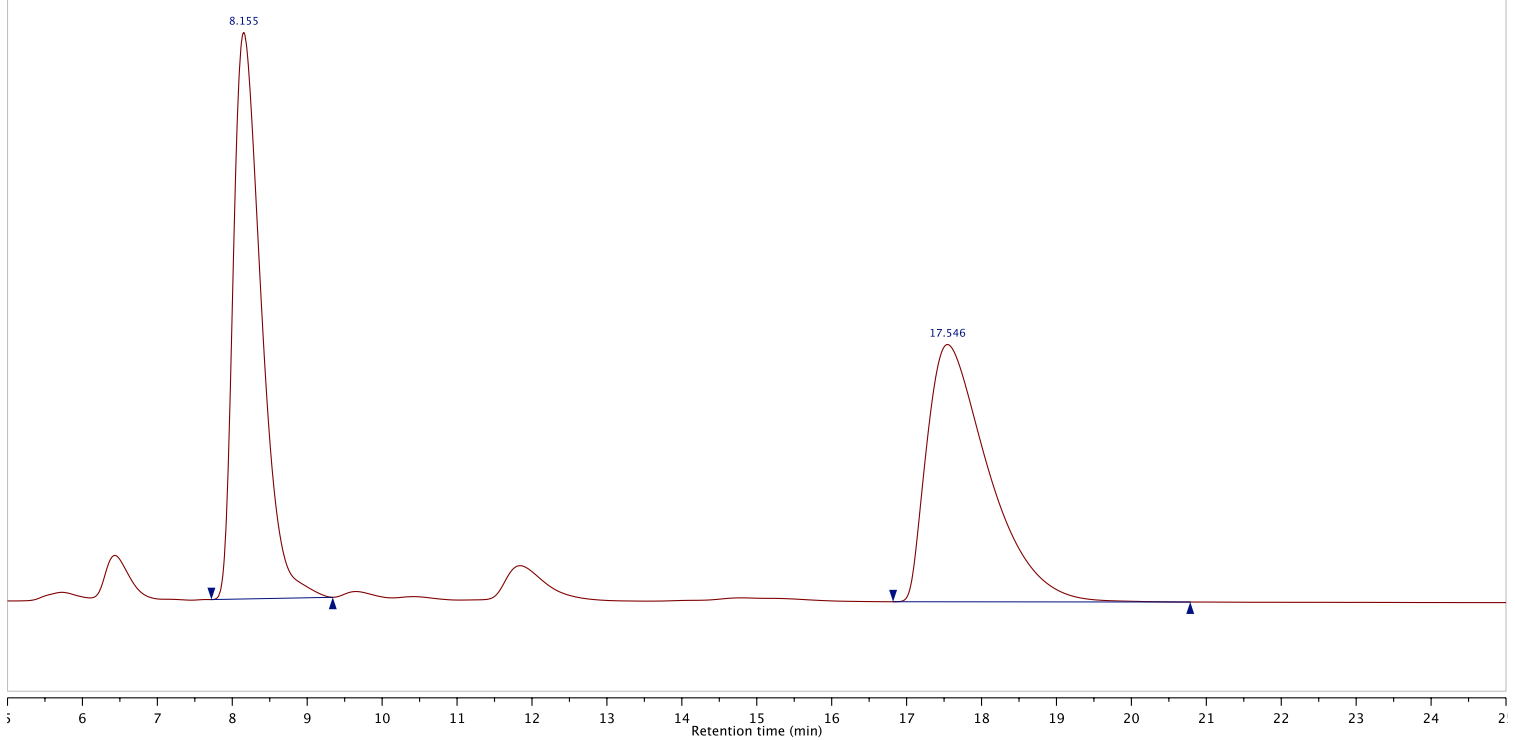

\section{Enantioenriched 9}

/Users/michael...-38-S1-3F-ER.D/ Injection 1 DAD1C, Sig=210,4 Ref=off Chromatogram

\begin{tabular}{|l|l|}
\hline \multicolumn{1}{|c|}{ RT } & Total Area \% \\
\hline 8.256 & 92.58 \\
\hline 18.353 & 7.42 \\
\hline
\end{tabular}

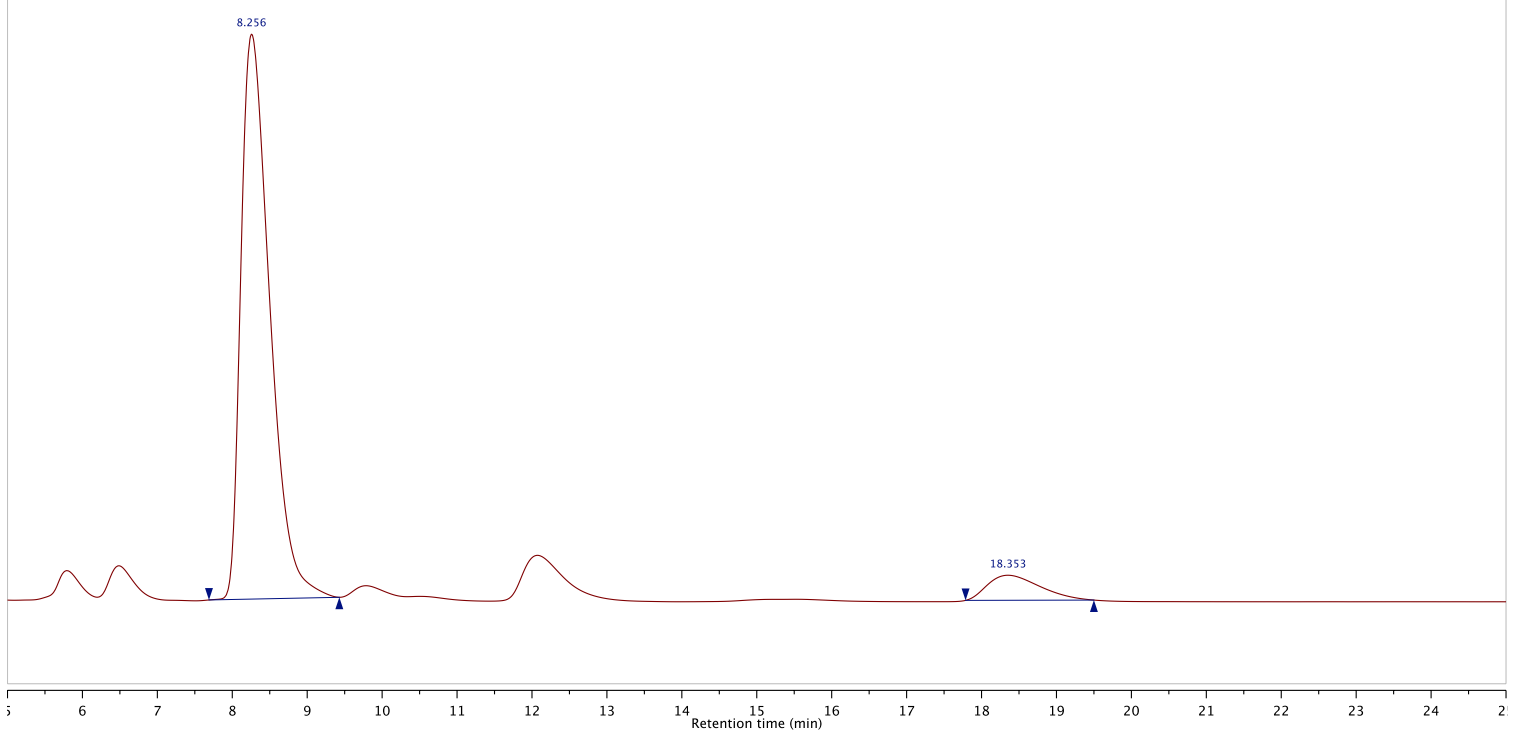




\section{Racemic 10}

IUsers/michael...18-44-2F-RAC.D/ Injection 1 DADIC, Sig=210,4 Ref=off Chromatogram

\begin{tabular}{|c|l|}
\hline RT & Total Area \% \\
\hline 8.839 & 49.75 \\
\hline 23.416 & 50.25 \\
\hline
\end{tabular}

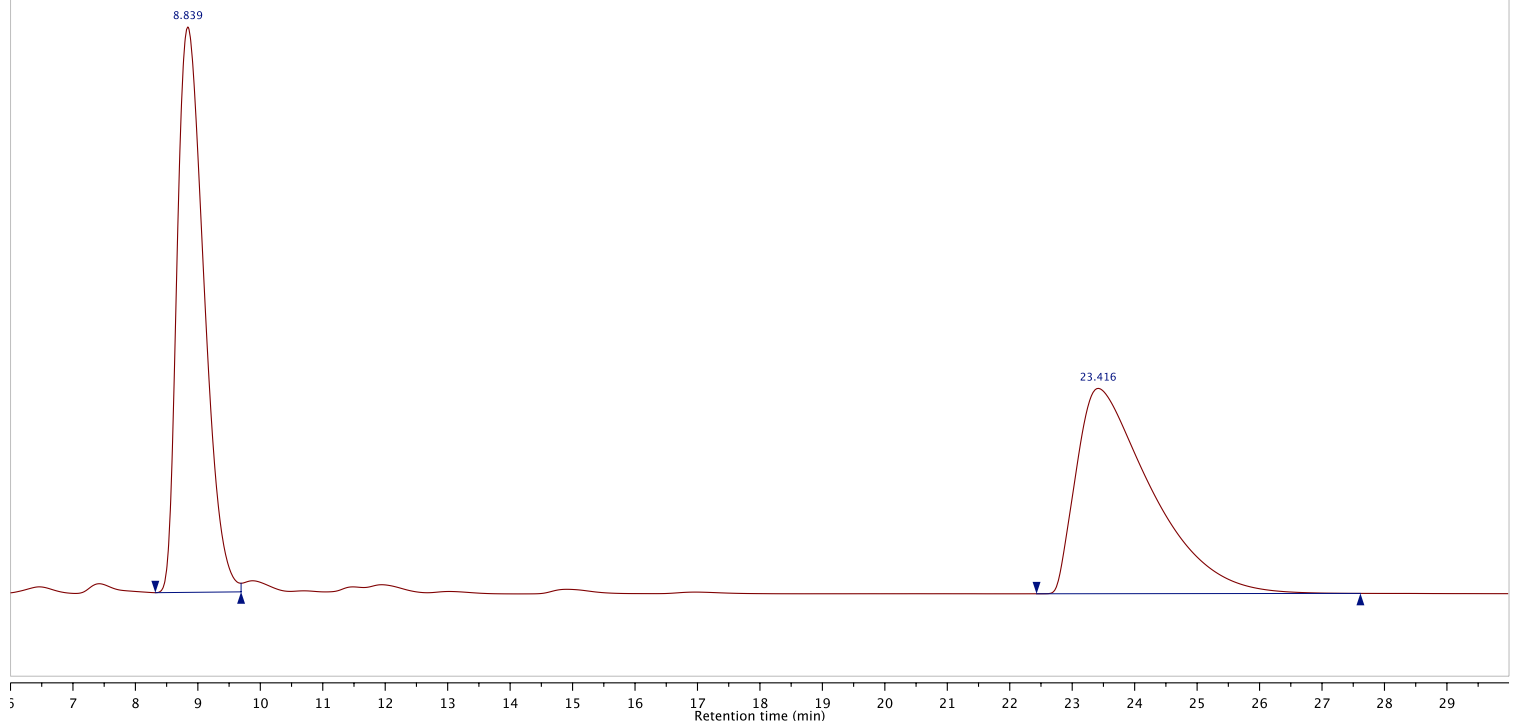

Enantioenriched 10

/Users/michael...-28-S1-2F-ER.D/ Injection 1 DAD1C, Sig=210,4 Ref=off Chromatogram

\begin{tabular}{|c|l|}
\hline RT & Total Area \% \\
\hline 24.302 & 11.11 \\
\hline 8.878 & 88.89 \\
\hline
\end{tabular}

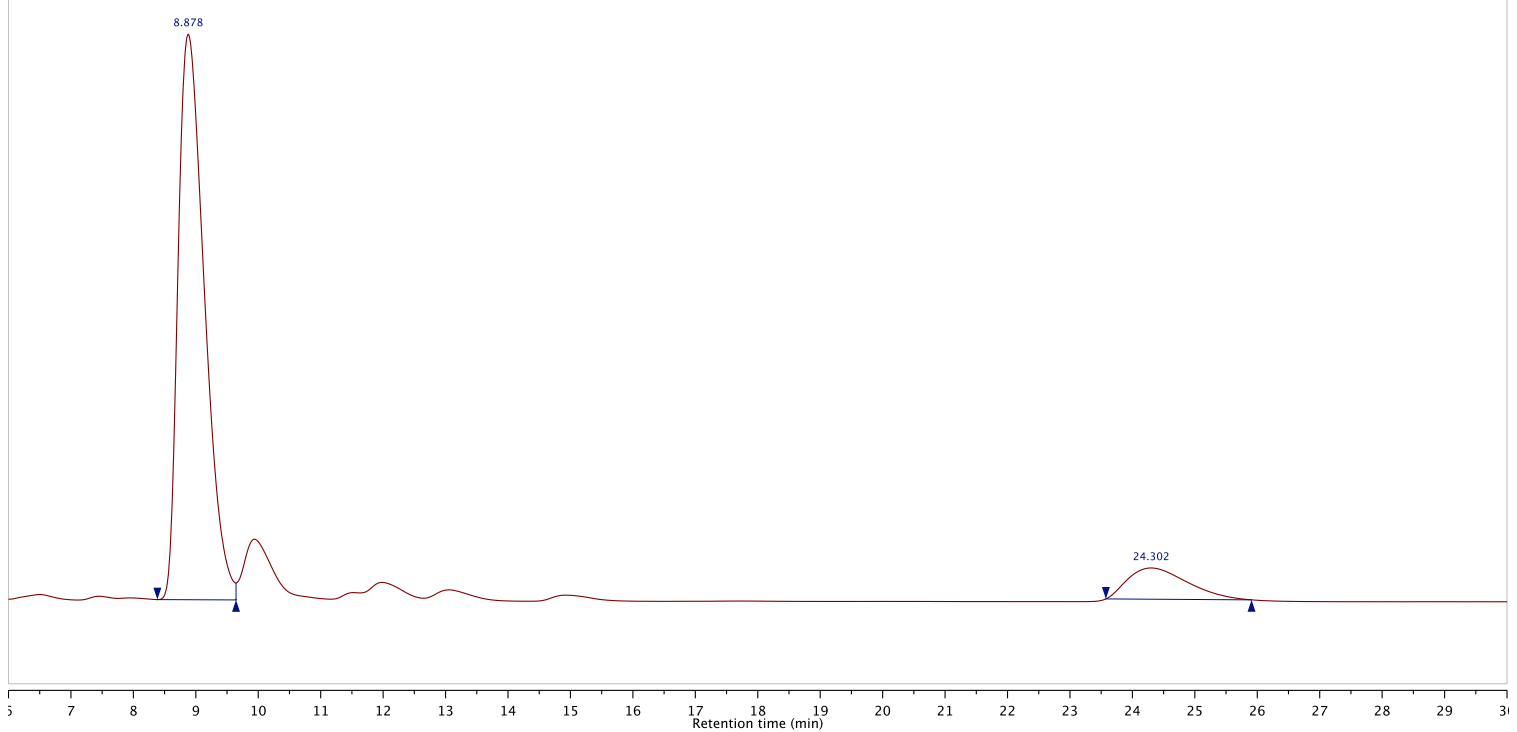




\section{Racemic 11}

/Users/michael...8-23-3CL-RAC.D/ Injection 1 DAD1D, Sig=230,4 Ref=off Chromatogram

\begin{tabular}{|c|l|}
\hline RT & Total Area \% \\
\hline 7.930 & 49.45 \\
\hline 17.361 & 50.55 \\
\hline
\end{tabular}

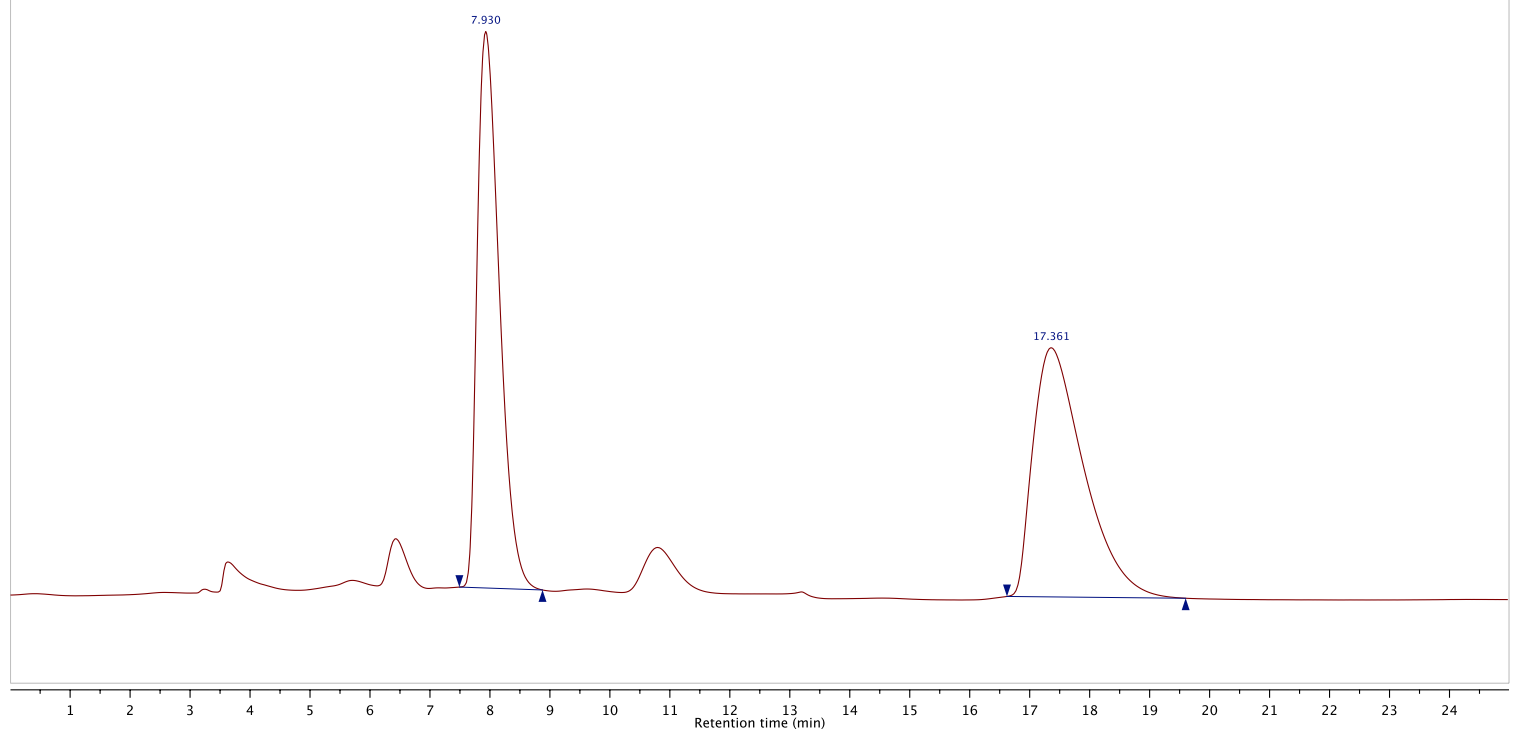

\section{Enantioenriched 11}

/Users/michael...23-10-3CL-ER.D/ Injection 1 DAD1D, Sig=230,4 Ref=off Chromatogram

\begin{tabular}{|l|l|}
\hline \multicolumn{1}{|c|}{ RT } & Total Area \% \\
\hline 8.108 & 92.90 \\
\hline 18.346 & 7.10 \\
\hline
\end{tabular}

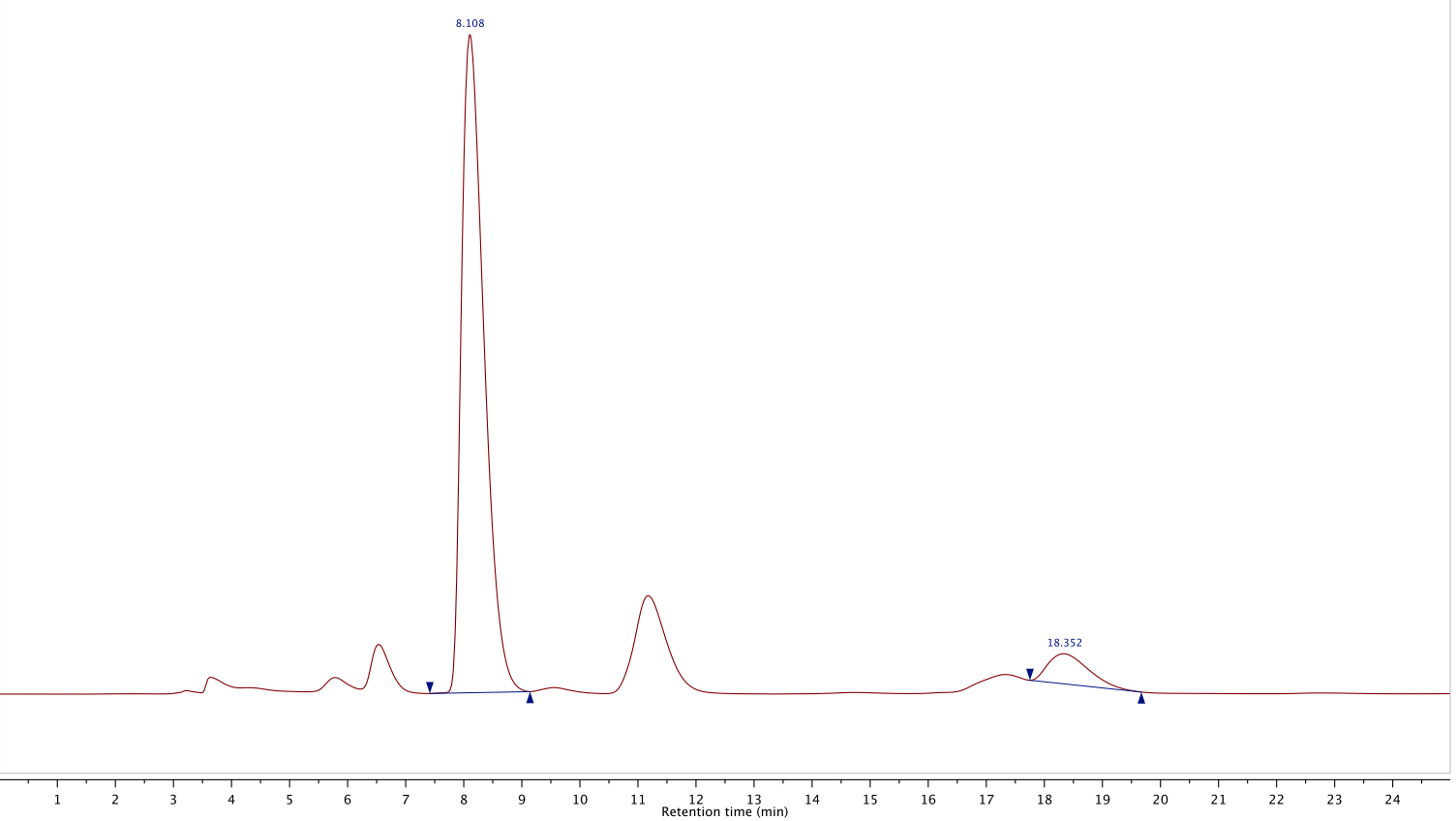




\section{Racemic 12}

IUsers/michael...-45-3NO2-RAC.D/ Injection 1 DAD1C, Sig=210,4 Ref=off Chromatogram

\begin{tabular}{|c|l|}
\hline RT & Total Area \% \\
\hline 12.217 & 51.12 \\
\hline 24.548 & 48.88 \\
\hline
\end{tabular}
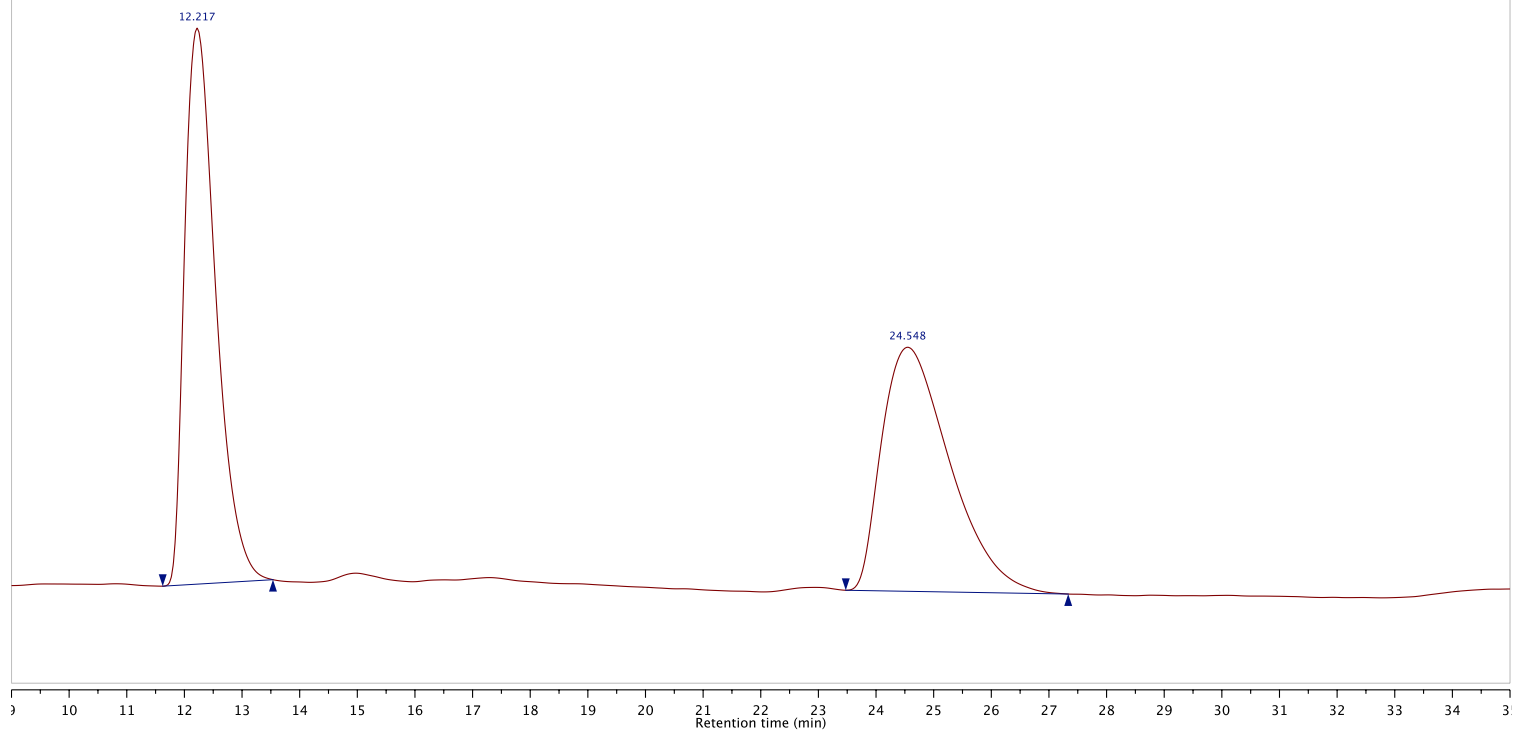

\section{Enantioenriched 12}

/Users/michael...9-S1-3NO2-ER.D/ Injection 1 DAD1C, Sig=210,4 Ref=off Chromatogram

\begin{tabular}{|c|l|}
\hline RT & Total Area \% \\
\hline 24.969 & 10.76 \\
\hline 12.457 & 89.24 \\
\hline
\end{tabular}
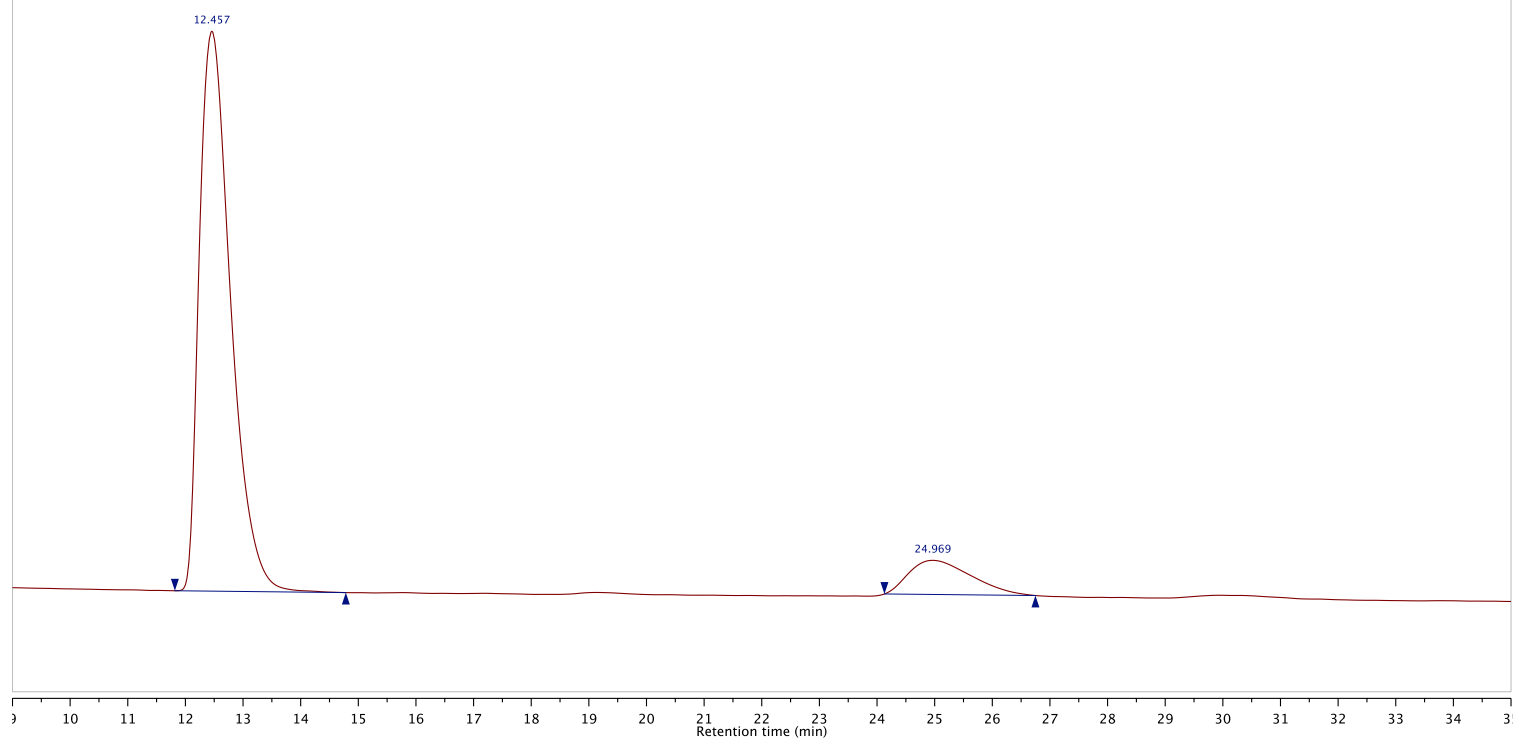


\section{Racemic 13}

/Users/michael...-46-THIO-RAC.D/ Injection 1 DAD1D, Sig=230,4 Ref=off Chromatogram

\begin{tabular}{|c|l|}
\hline RT & Total Area \% \\
\hline 15.193 & 49.95 \\
\hline 36.943 & 50.05 \\
\hline
\end{tabular}

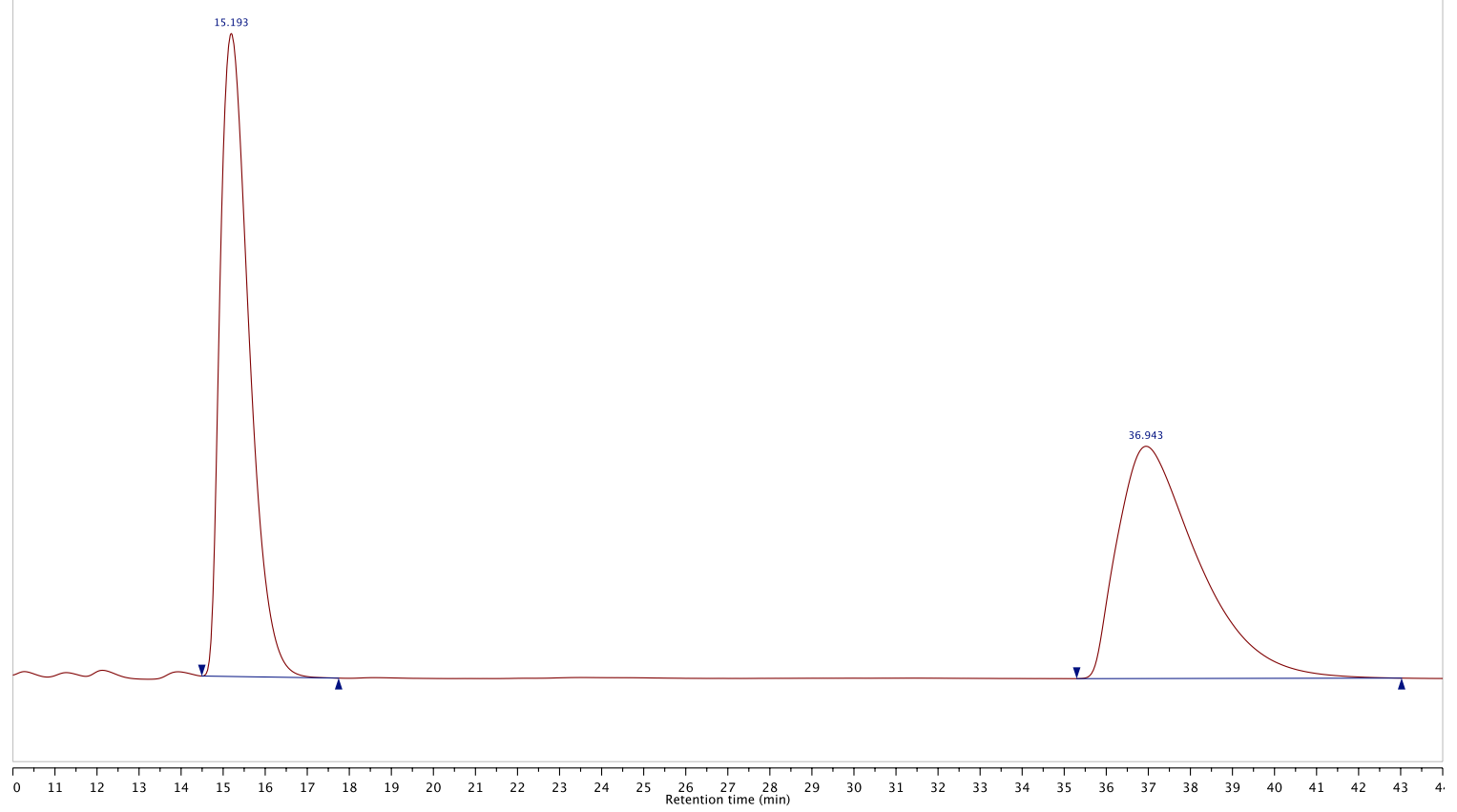

\section{Enantioenriched 13}

/Users/michael...7-S1-THIO-ER.D/ Injection 1 DAD1D, Sig=230,4 Ref=off Chromatogram

\begin{tabular}{|c|l|}
\hline RT & Total Area \% \\
\hline 14.507 & 94.76 \\
\hline 34.064 & 5.24 \\
\hline
\end{tabular}
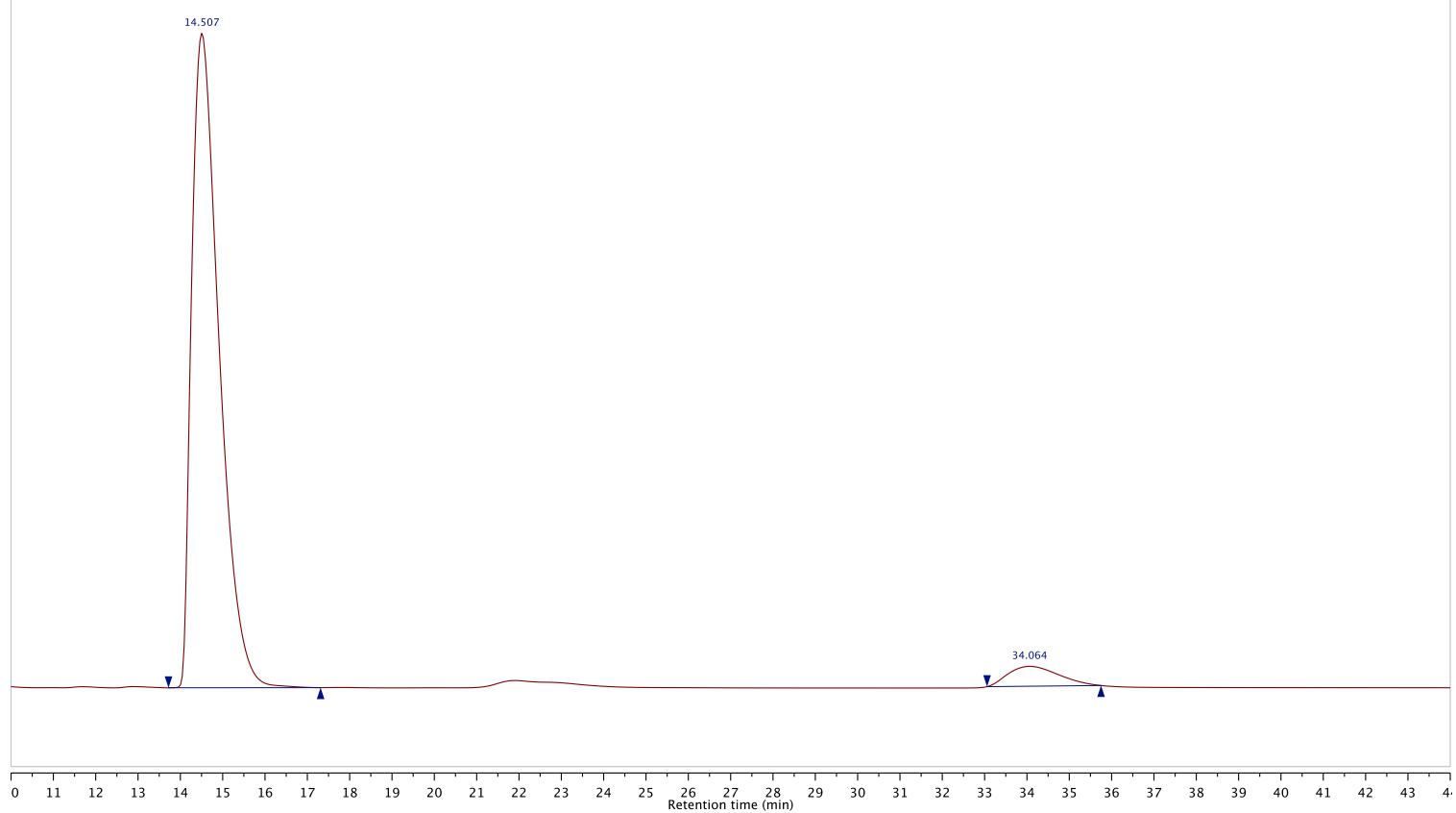


\section{Racemic 14}

/Users/michael...44-S1-BN-RAC.D/ Injection 1 DAD1C, Sig=210,4 Ref=off Chromatogram

\begin{tabular}{|c|l|}
\hline RT & Total Area \% \\
\hline 12.699 & 50.96 \\
\hline 18.098 & 49.04 \\
\hline
\end{tabular}

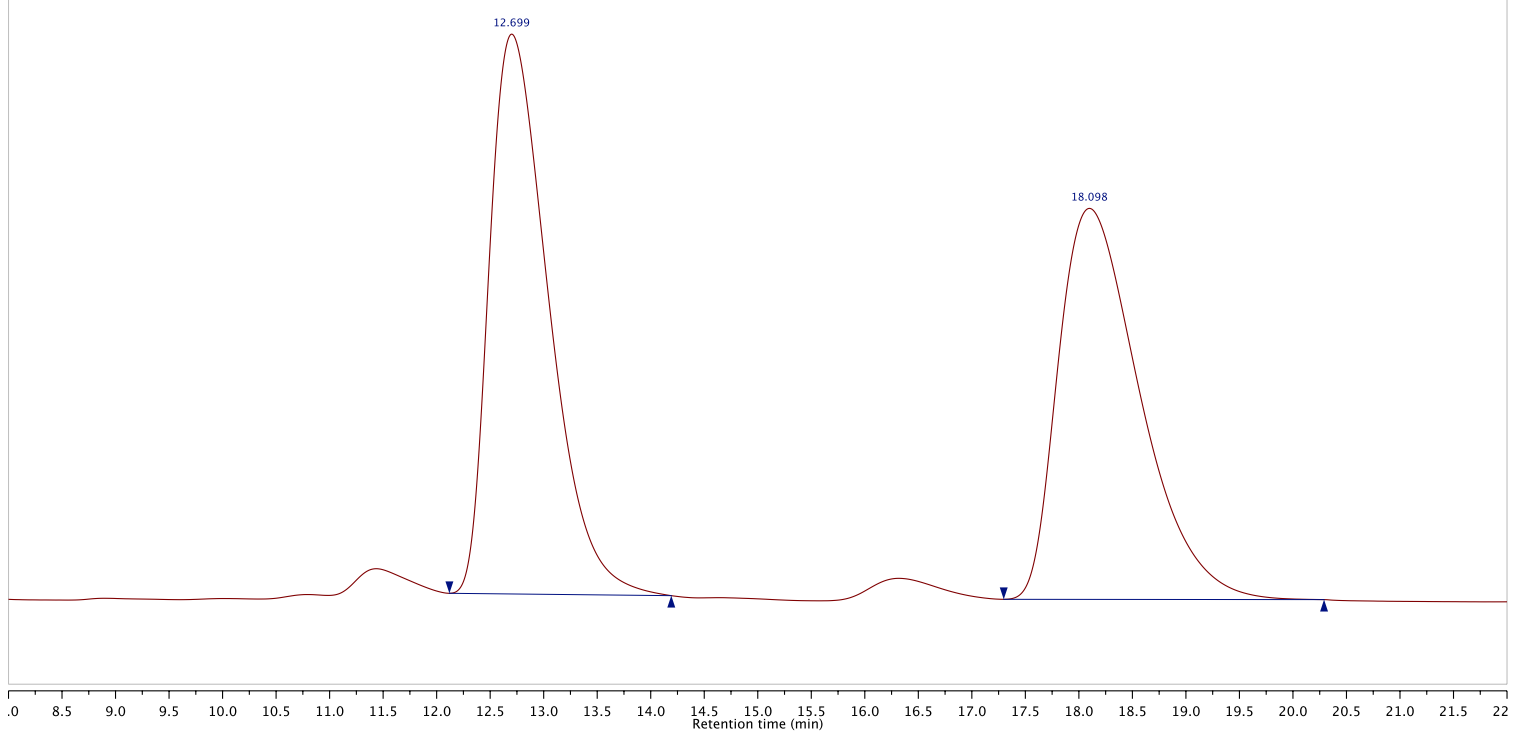

\section{Enantioenriched 14}

/USers/michael...-25-41-EN-ER.D/ Injection 1 DAD1C, Sig=210,4 Ref=off Chromatogram

\begin{tabular}{|c|l|}
\hline RT & Total Area \% \\
\hline 12.635 & 91.55 \\
\hline 18.081 & 8.45 \\
\hline
\end{tabular}

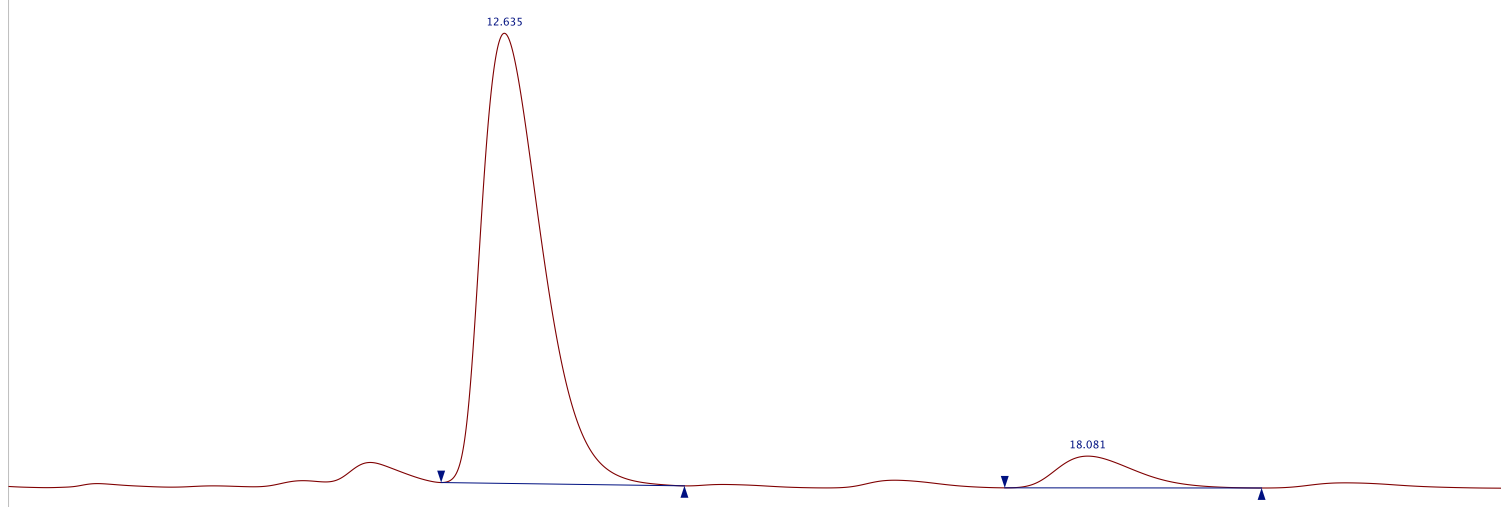

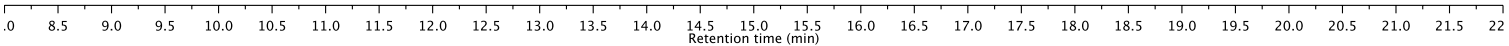




\section{Racemic 15}

UUsers/michael...51-MEO2C-RAC.D/Injection 1 DAD C, Sig $=210,8$ Ref $=360,100$ Chromatogram

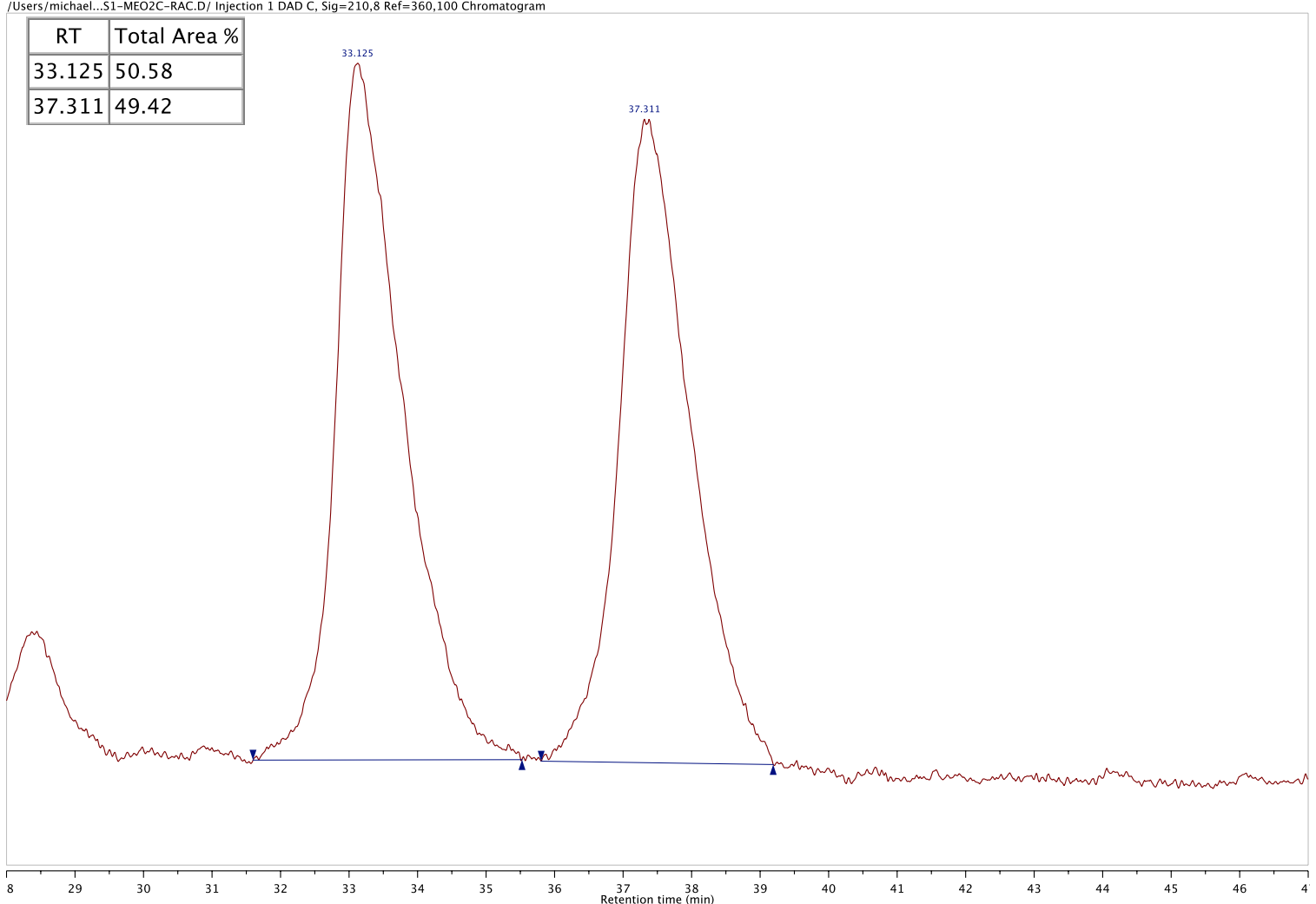

\section{Enantioenriched 15}

/Users/michael...-S1-MEO2C-ER.D/ Injection 1 DAD C, Sig=210,8 Ref=360,100 Chromatogram

\begin{tabular}{|c|l|}
\hline RT & Total Area \% \\
\hline 32.796 & 10.15 \\
\hline 36.035 & 89.85 \\
\hline
\end{tabular}




\section{Racemic 16}

IUsers/michael...11-BNO2C-RAC.D/ Injection 1 DAD C, Sig=210,8 Ref=360,100 Chromatogram

\begin{tabular}{|c|l|}
\hline RT & Total Area \% \\
\hline 55.078 & 50.00 \\
\hline 63.284 & 50.00 \\
\hline
\end{tabular}

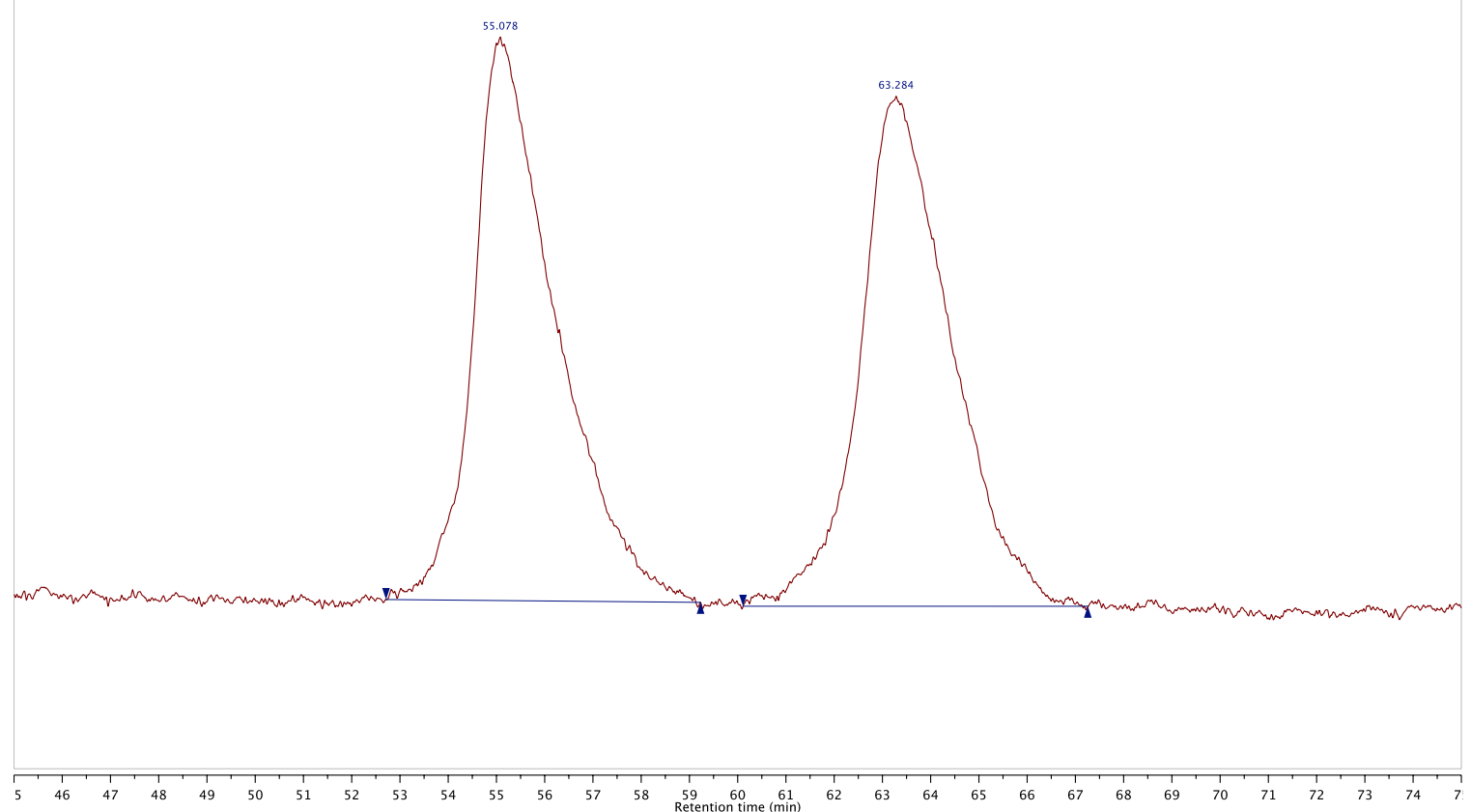

Enantioenriched 16

/Users/michael...-S1-BNO2C-ER.D/ Injection 1 DAD C, Sig=210,8 Ref=360,100 Chromatogram

\begin{tabular}{|c|l|}
\hline RT & Total Area \% \\
\hline 55.209 & 4.63 \\
\hline 61.242 & 95.37 \\
\hline
\end{tabular}

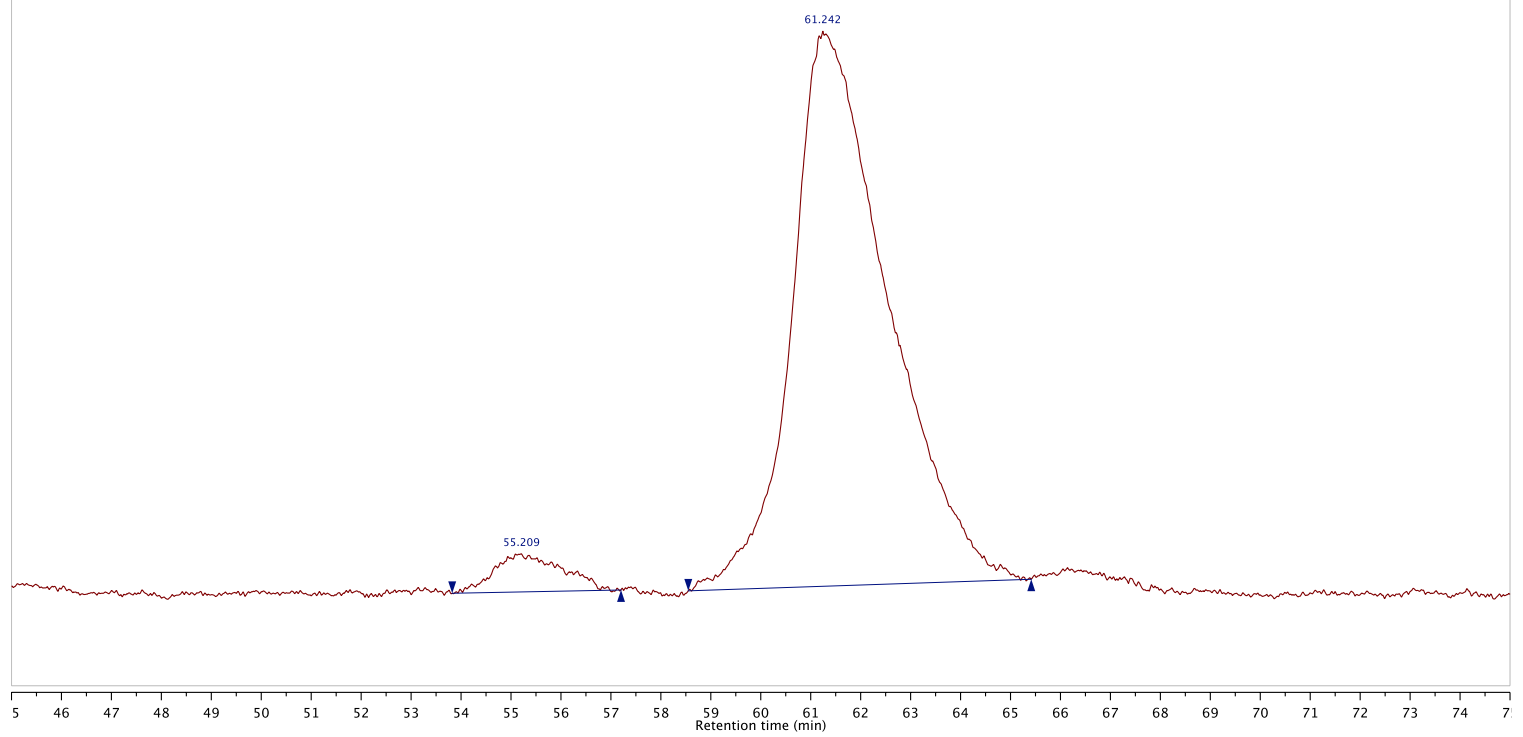




\section{Racemic 17}

IUsers/michael...TBUO2C-RAC-E.D/Injection 1 DAD1C, Sig=210,4 Ref=off Chromatogram

\begin{tabular}{|c|l|}
\hline RT & Total Area \% \\
\hline
\end{tabular}

7.64949 .30

\begin{tabular}{l|l|l}
7.329 & 50.70
\end{tabular}

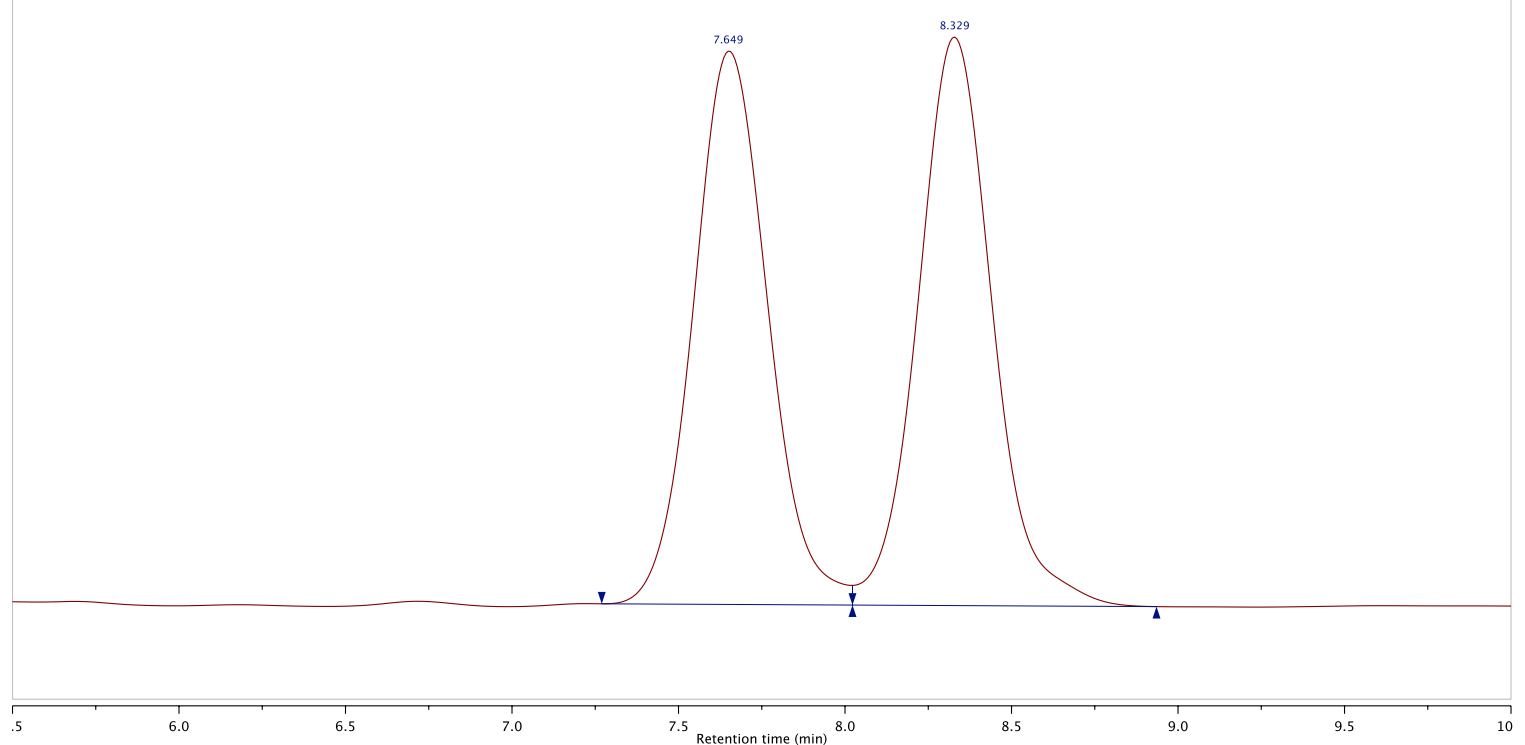

Enantioenriched 17

/Users/michael...-TBUO2C-ER-B.D/ Injection 1 DAD1C, Sig=210,4 Ref=off Chromatogram

\begin{tabular}{|c|c|}
\hline RT & Total Area $\%$ \\
\hline 8.260 & 96.58 \\
\hline
\end{tabular}

\begin{tabular}{l|l|l}
\hline 8.260 & 96.58
\end{tabular}

\begin{tabular}{|l|l|l|}
\hline 7.573 & 3.42 \\
\hline
\end{tabular}

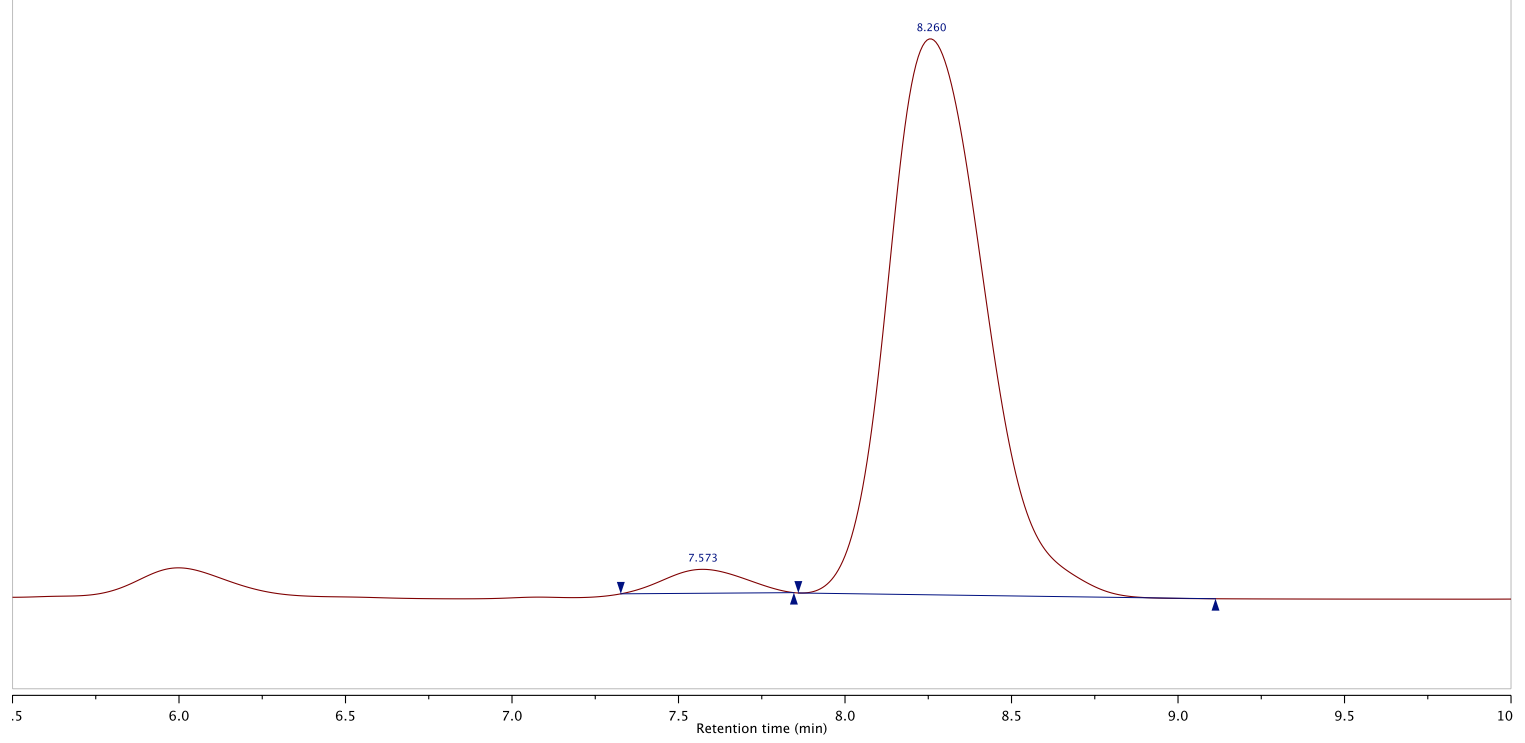




\section{Racemic 18}

/Users/michael...-MEOC-RAC-A2.D/ Injection 1 DAD1C, Sig=210,4 Ref=off Chromatogram

\begin{tabular}{|c|l|}
\hline RT & Total Area \% \\
\hline 8.686 & 49.41 \\
\hline 11.119 & 50.59 \\
\hline
\end{tabular}

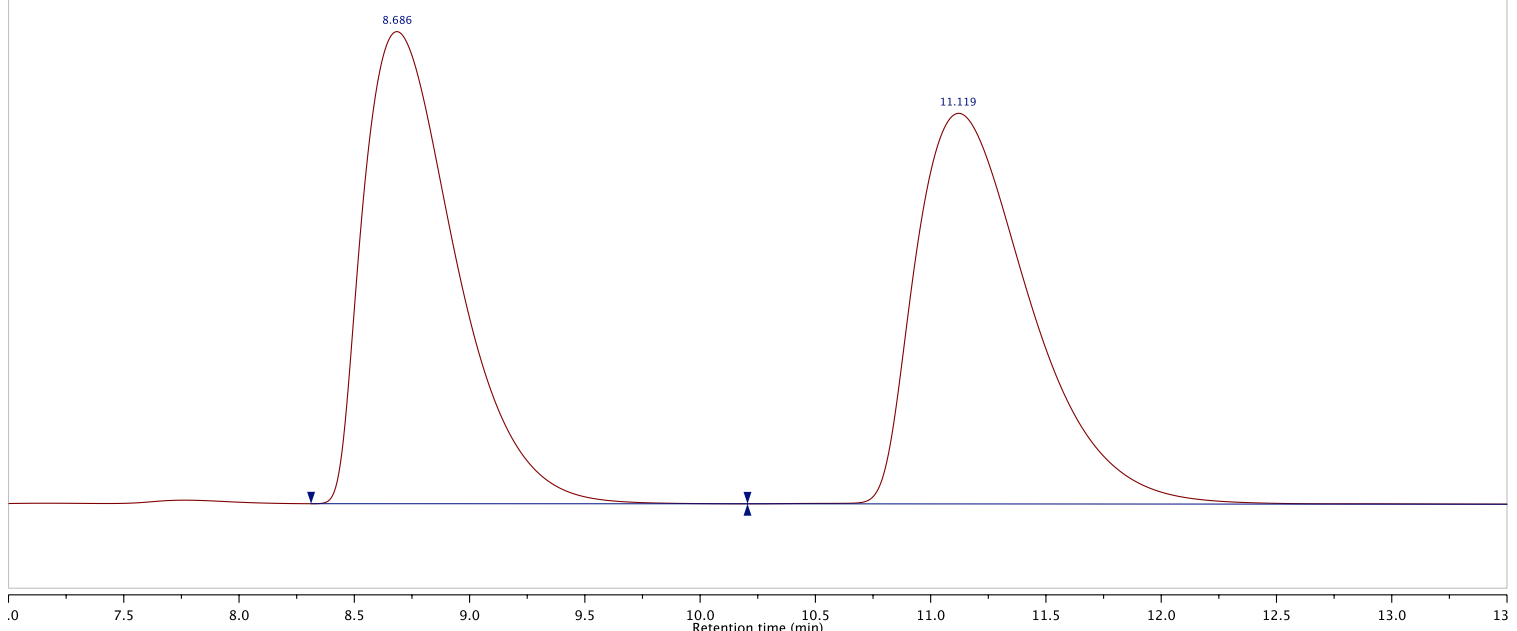

Enantioenriched 18

/USers/michael...1-S1-MEOC-ER.D/ Injection 1 DAD1C, Sig=210,4 Ref=off Chromatogram

\begin{tabular}{|c|l|}
\hline RT & Total Area \% \\
\hline 10.746 & 82.80 \\
\hline 8.580 & 17.20 \\
\hline
\end{tabular}

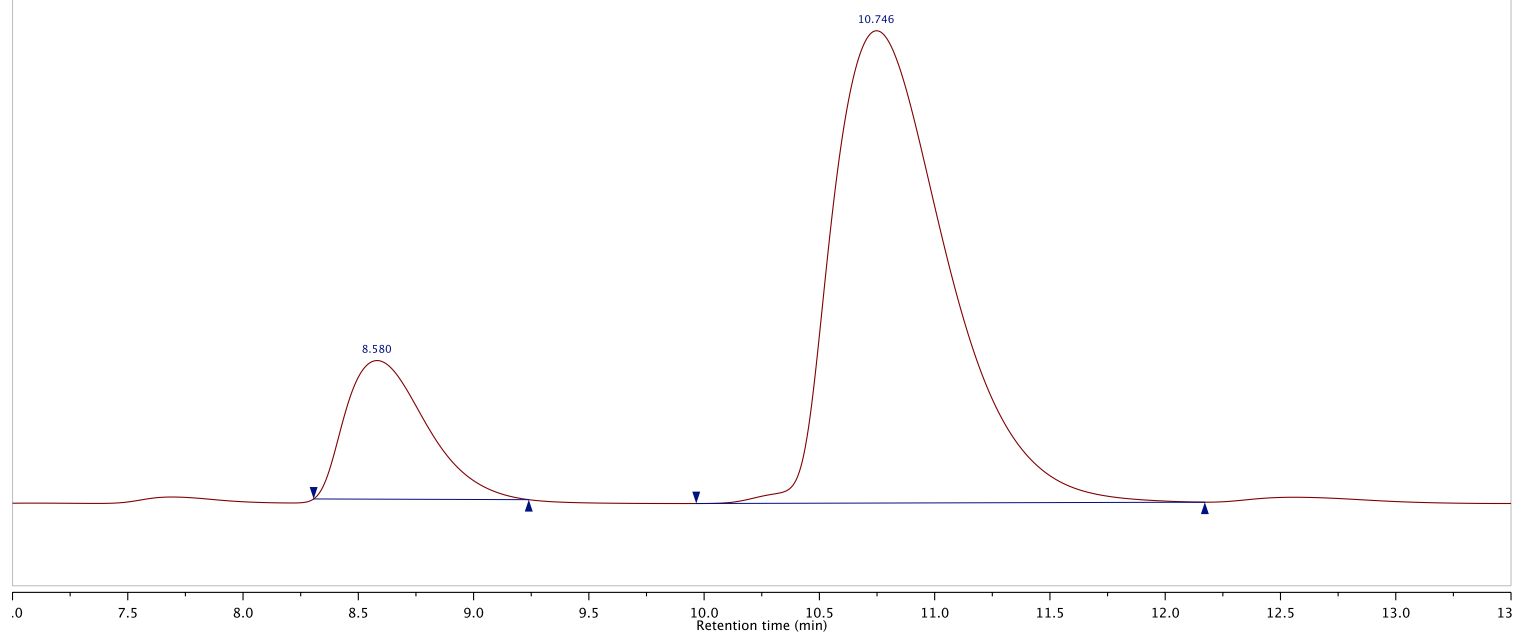




\section{Racemic 19}

IUsers/michael...-14-MEOH-RAC.D/ Injection 1 DAD1C, Sig=210,4 Ref=off Chromatogram

\begin{tabular}{|c|l|}
\hline RT & Total Area \% \\
\hline 15.928 & 49.91 \\
\hline 27.006 & 50.09 \\
\hline
\end{tabular}

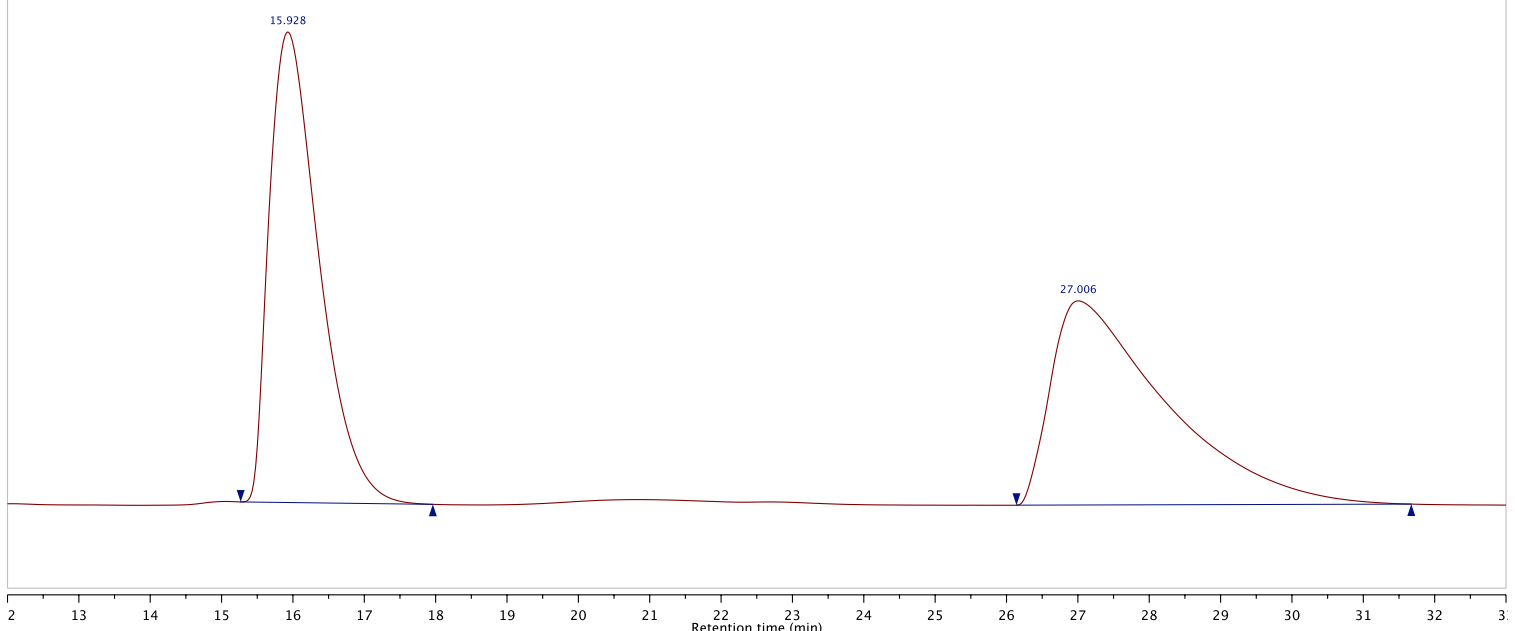

\section{Enantioenriched 19}

/USers/michael...1-S1-MEOH-ER.D/ Injection 1 DAD1C, Sig=210,4 Ref=off Chromatogram

\begin{tabular}{|c|l|}
\hline RT & Total Area \% \\
\hline
\end{tabular}

\begin{tabular}{|l|l|}
\hline 28.280 & 8.72 \\
\hline
\end{tabular}

15.78991 .28

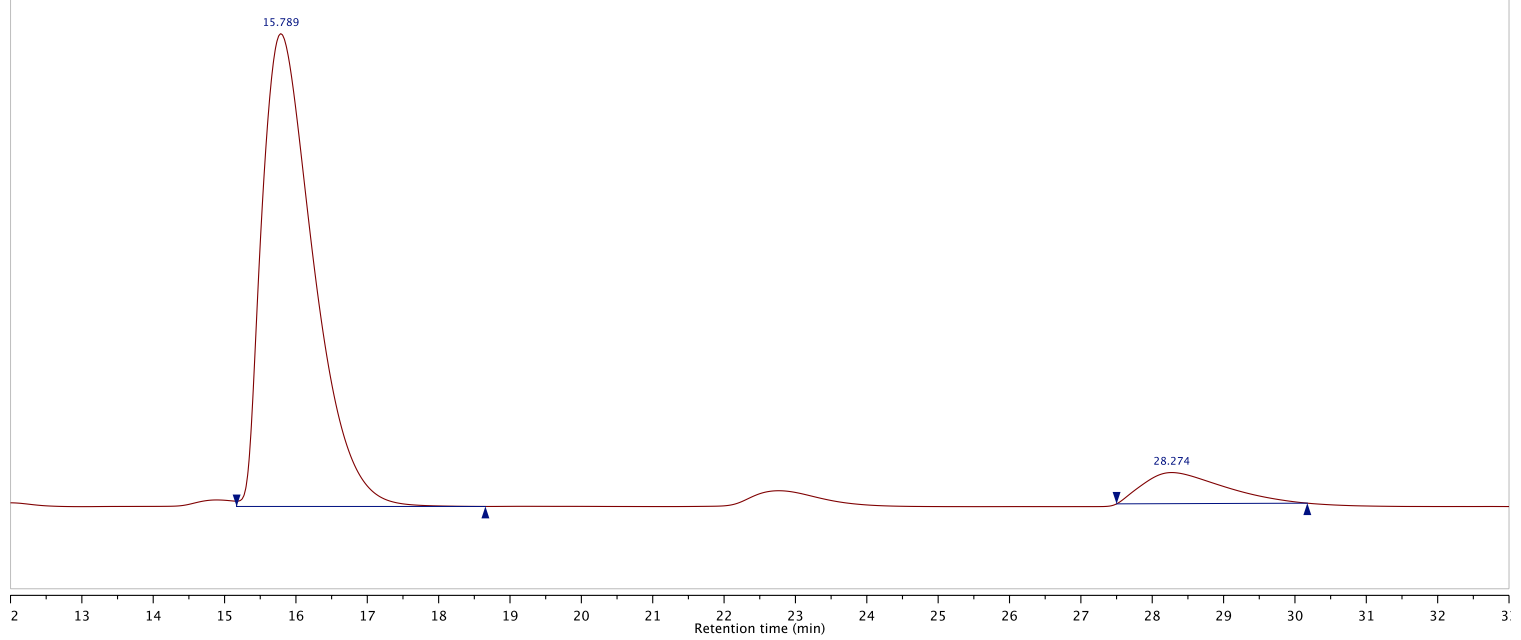




\section{Racemic 20}

IUsers/michael...-45-BNOH-RAC.D/ Injection 1 DAD C, Sig=210,8 Ref=360,100 Chromatogram

\begin{tabular}{|c|l|}
\hline RT & Total Area \% \\
\hline 21.063 & 50.03 \\
\hline 23.756 & 49.97 \\
\hline
\end{tabular}

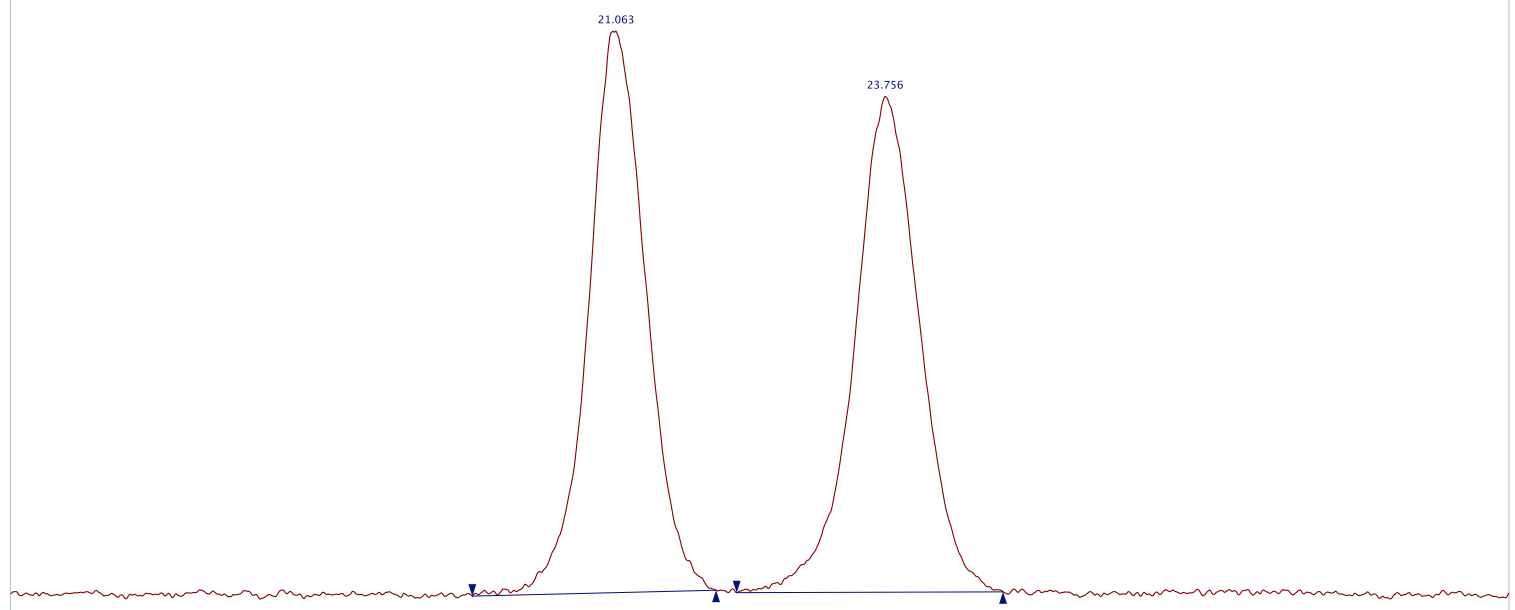

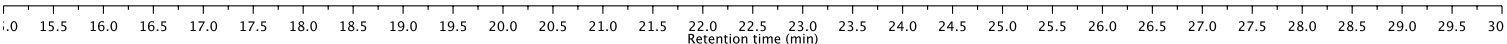
Enantioenriched 20

/Users/michael...3-77-BNOH-ER.D/ Injection 1 DAD C, sig=210,8 Ref=360,100 Chromatogram

\begin{tabular}{|c|c|c|}
\hline RT & Total Area \% \\
\hline
\end{tabular}

23.17992 .69

\begin{tabular}{|l|l|l}
20.733 & 7.31
\end{tabular}

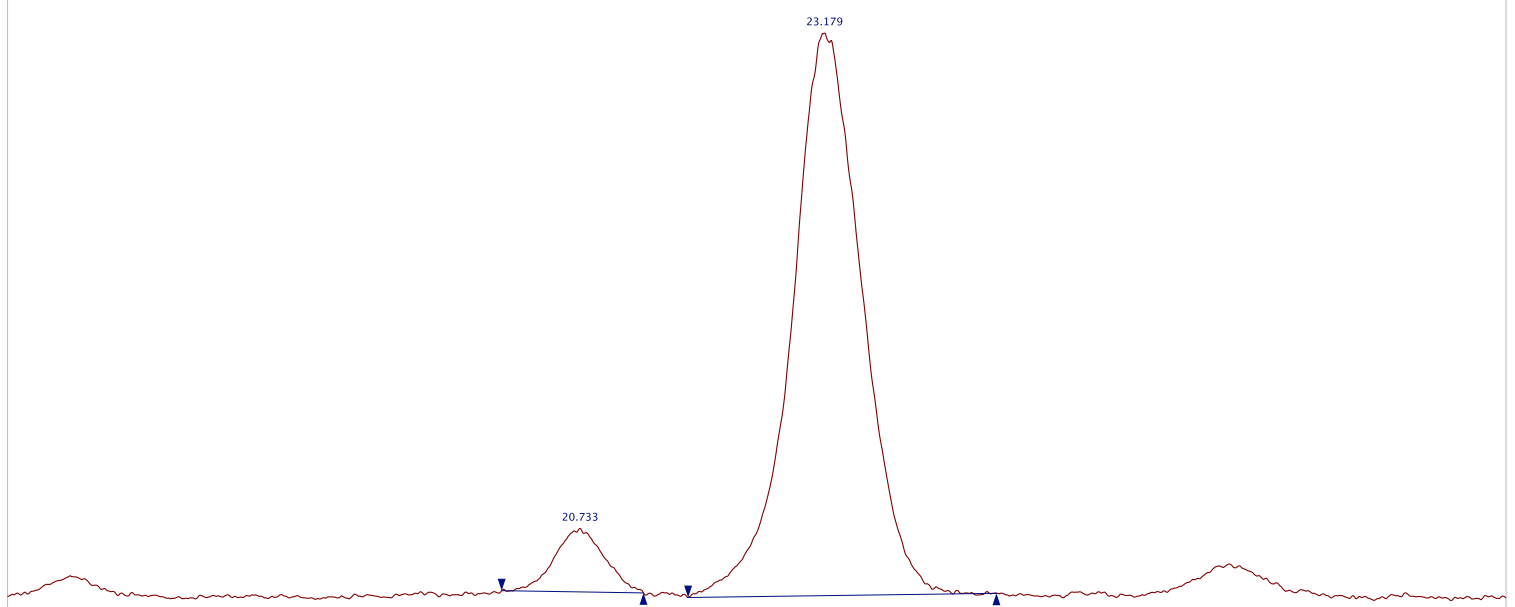

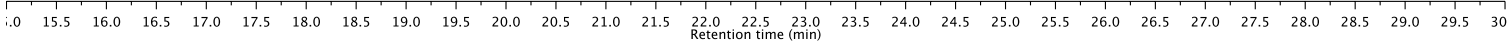




\section{Racemic 21}

/Users/michael...S1-TMS-RAC-D.D/ Injection 1 DADIC, Sig=210,4 Ref=off Chromatogram

\begin{tabular}{|c|l|}
\hline RT & Total Area \% \\
\hline 8.450 & 50.46 \\
\hline 11.197 & 49.54 \\
\hline
\end{tabular}

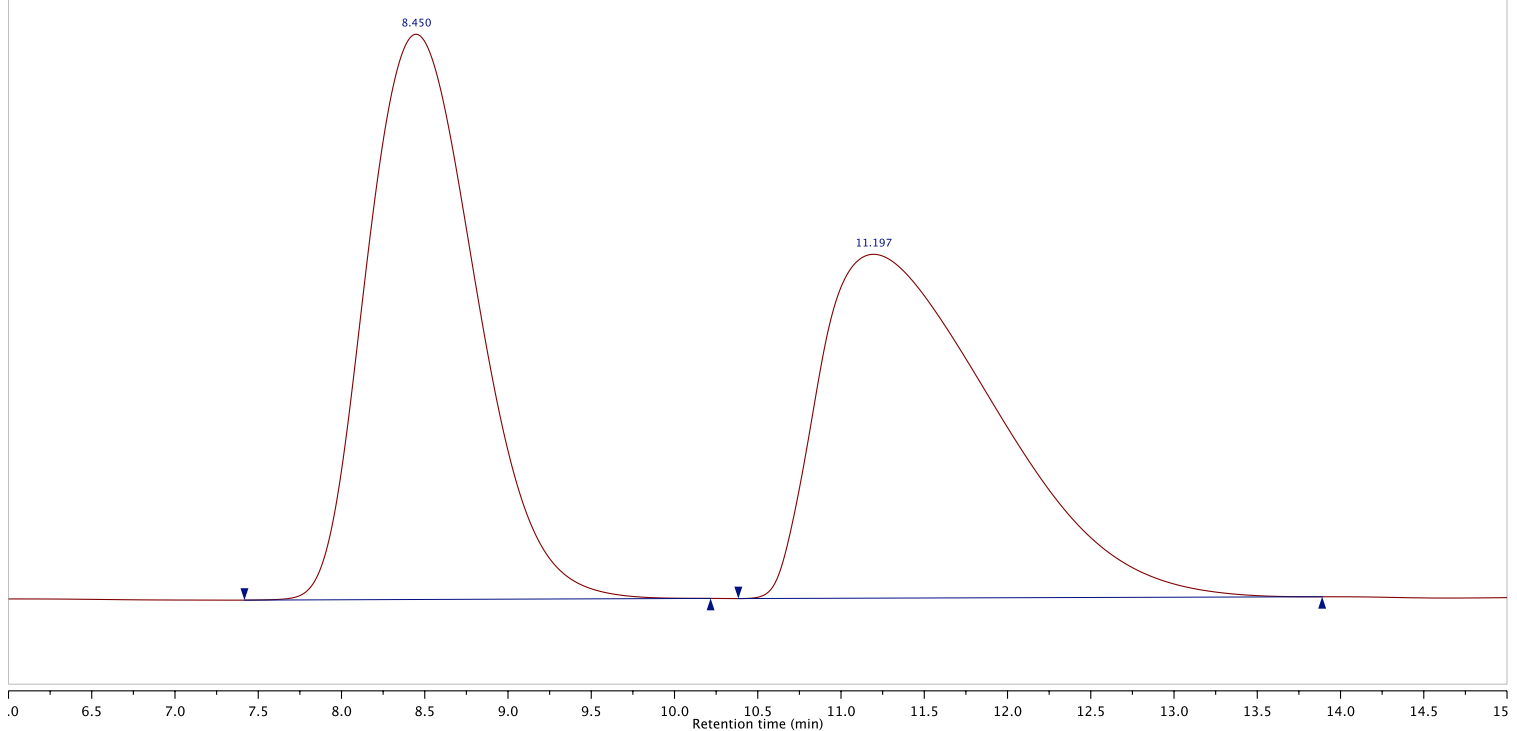

\section{Enantioenriched 21}

/Users/michael...-S1-TMS-ER-B.D/ Injection 1 DAD1C, Sig=210,4 Ref=off Chromatogram

\begin{tabular}{|l|l|}
\hline \multicolumn{1}{|c|}{ RT } & Total Area \% \\
\hline 11.377 & 90.72 \\
\hline 8.477 & 9.28 \\
\hline
\end{tabular}

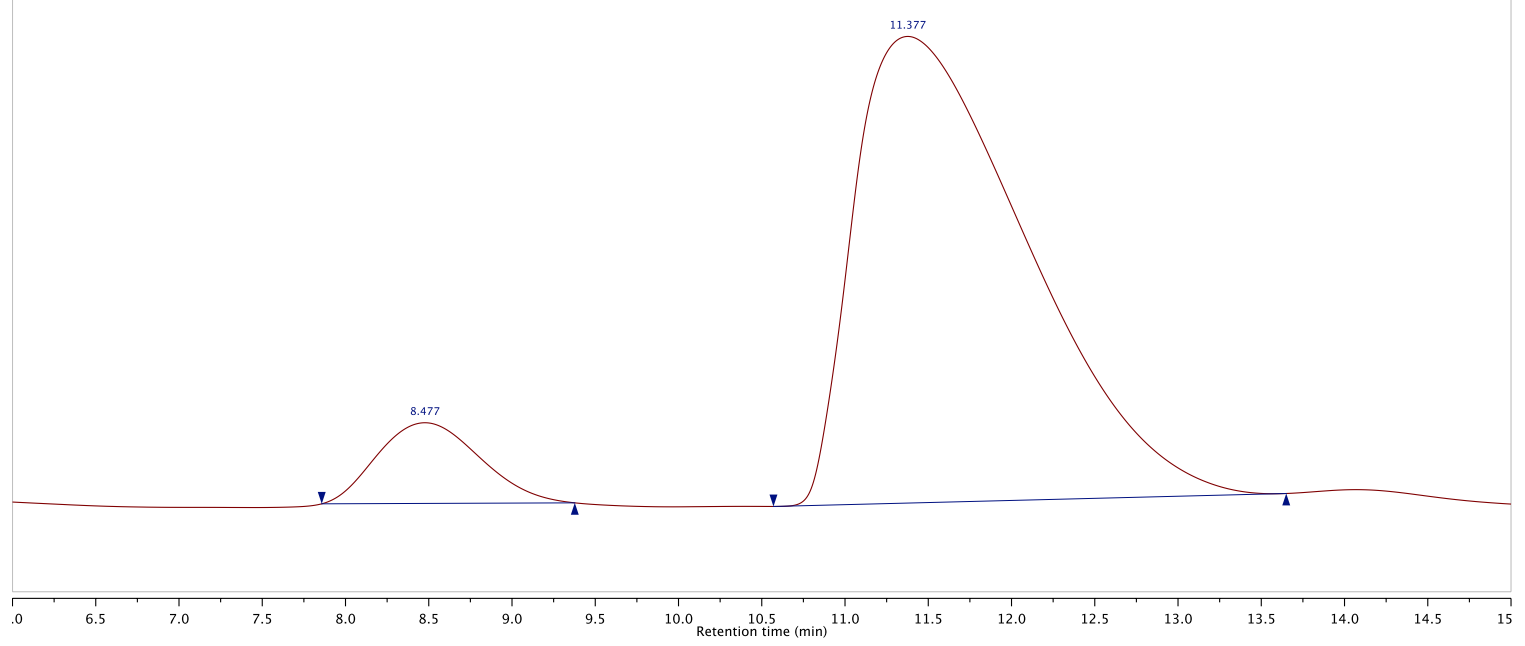




\section{Racemic 22}

IUsers/michael...-4BRBNOH-RAC.D/ Injection 1 DAD D, Sig=230,16 Ref=360,100 Chromatogram

\begin{tabular}{|c|l|}
\hline RT & Total Area \% \\
\hline 22.921 & 50.50 \\
\hline 26.987 & 49.50 \\
\hline
\end{tabular}

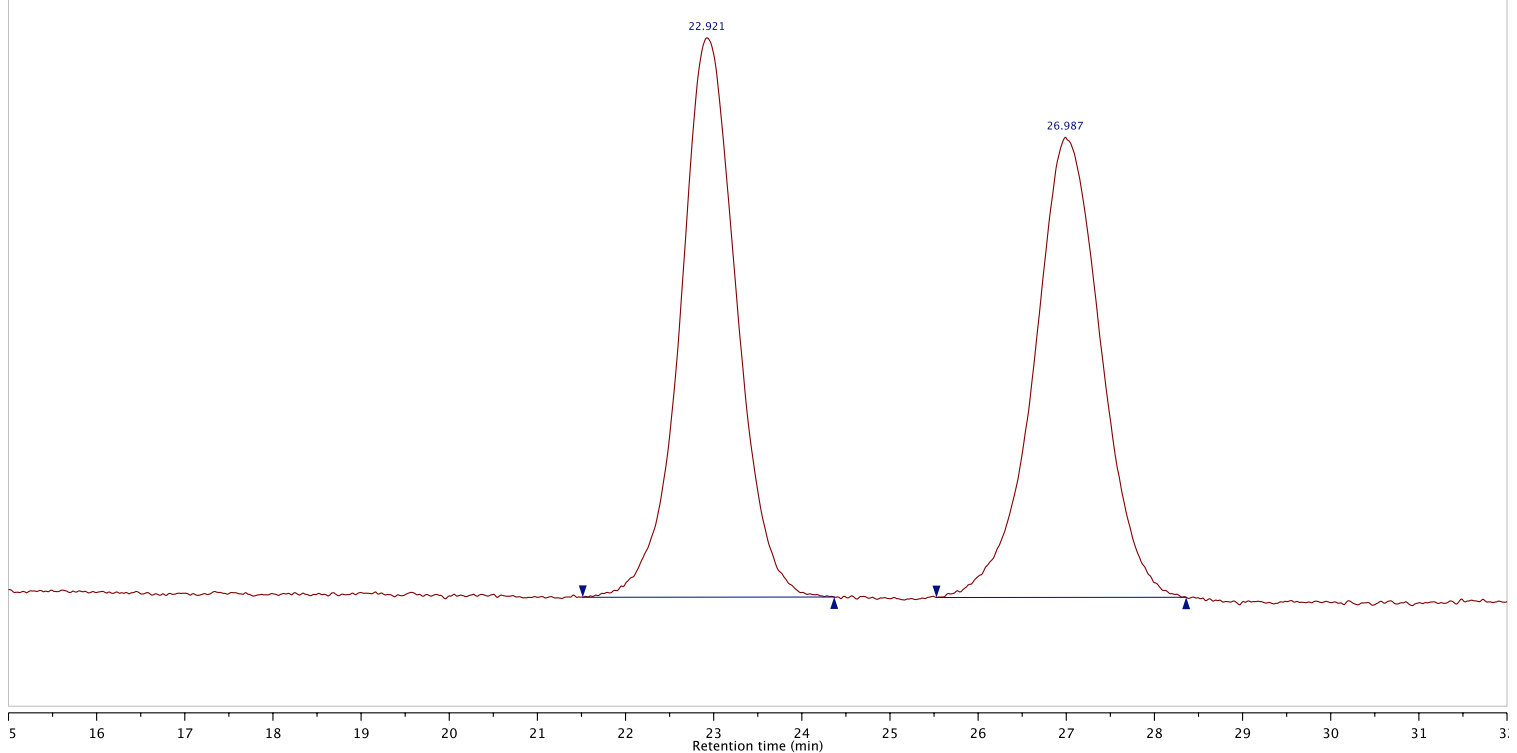

Enantioenriched 22

/USers/michael...1-4BRBBNOH-ER.D/ Injection 1 DAD D, Sig=230,16 Ref=360,100 Chromatogram

\begin{tabular}{|c|l|}
\hline RT & Total Area \% \\
\hline
\end{tabular}

\begin{tabular}{|l|l|}
\hline 22.889 & 7.13
\end{tabular}

\begin{tabular}{ll|l|l}
26.575 & 92.87
\end{tabular}

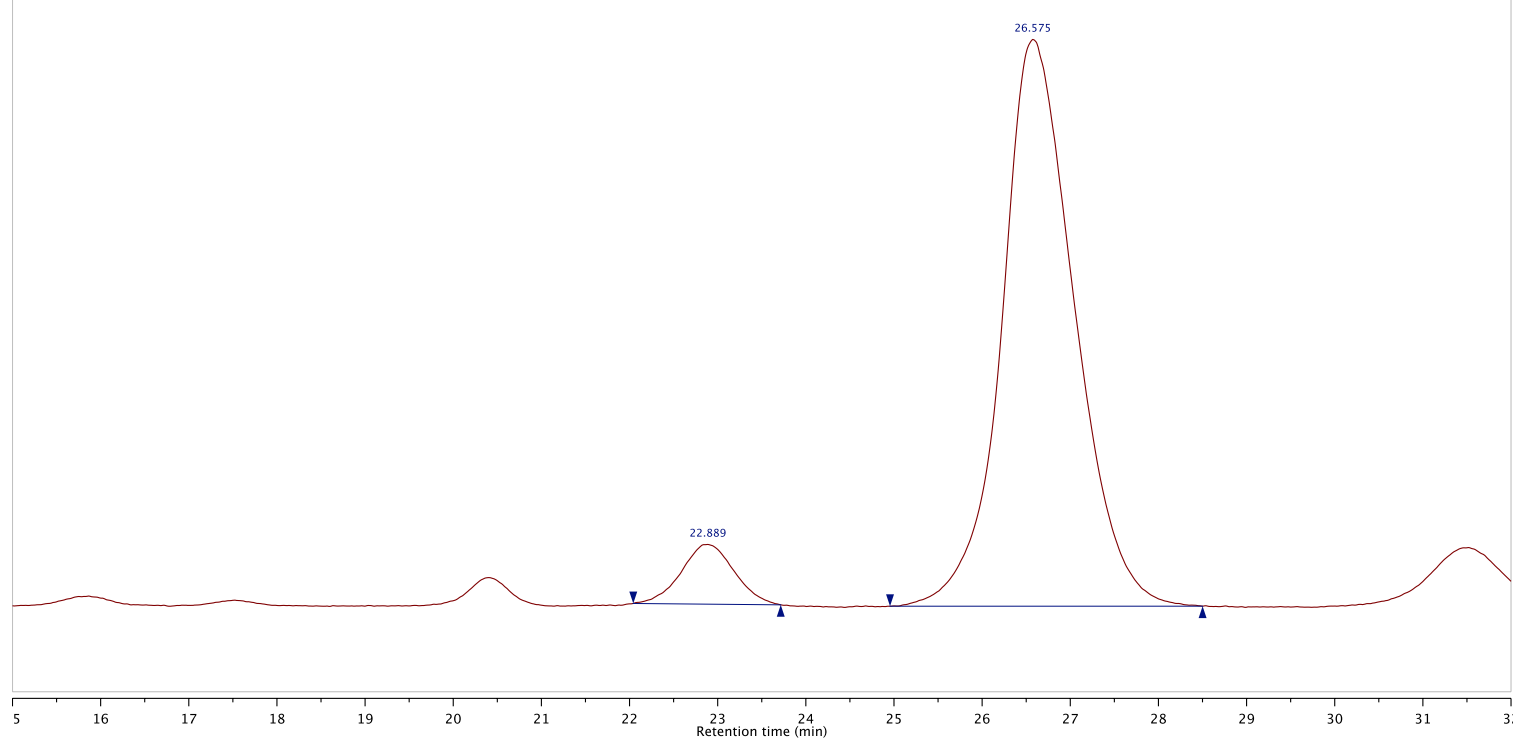




\section{Racemic 4}

IUsers/michael...5-28-4ME-RAC.D/ Injection 1 DAD1C, Sig=210,4 Ref=off Chromatogram

\begin{tabular}{|c|c|c|}
\hline RT & Total Area \% \\
\hline
\end{tabular}

6.98149 .85

8.56150 .15

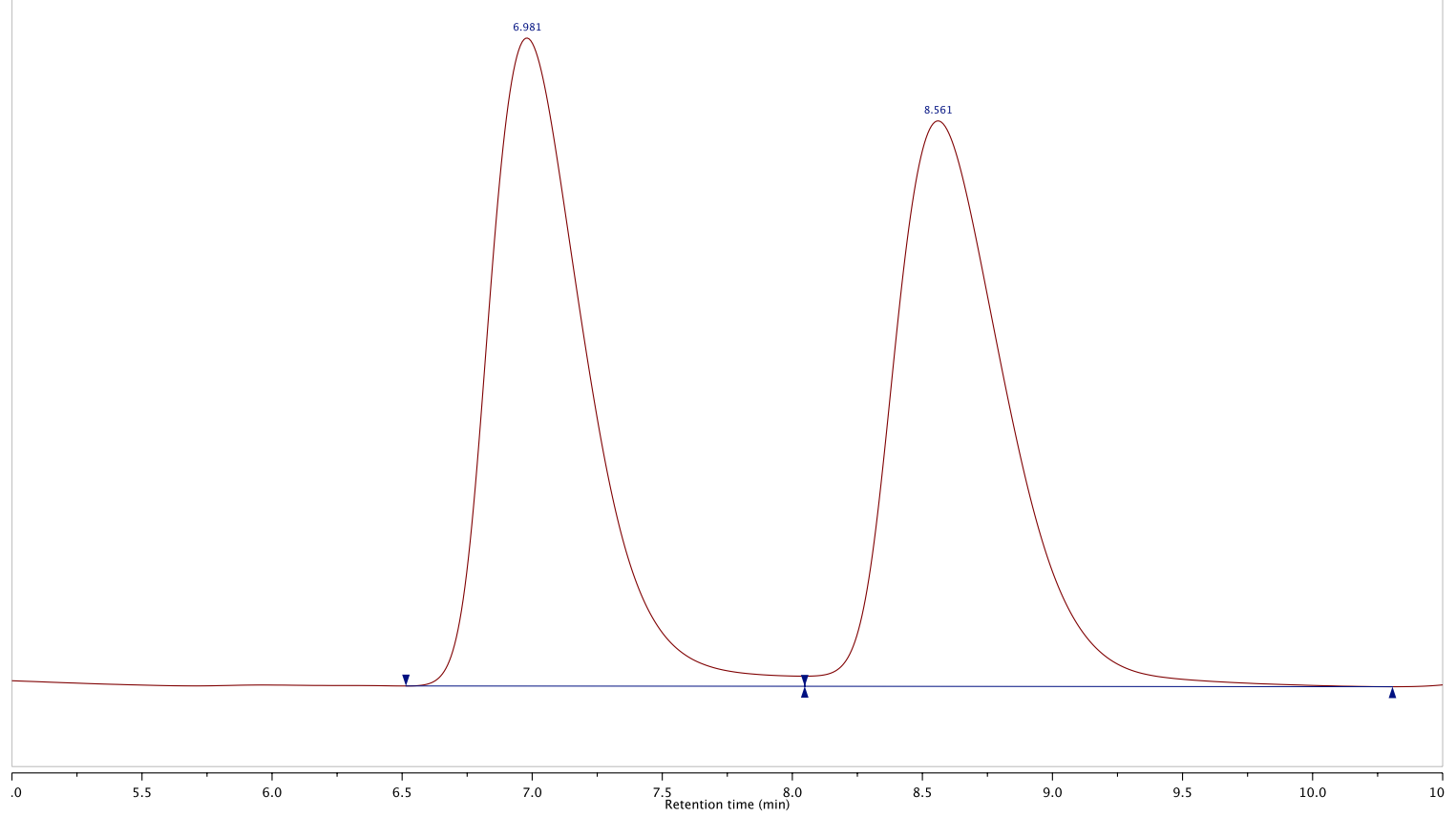

Enantioenriched 4 (from general procedure B)

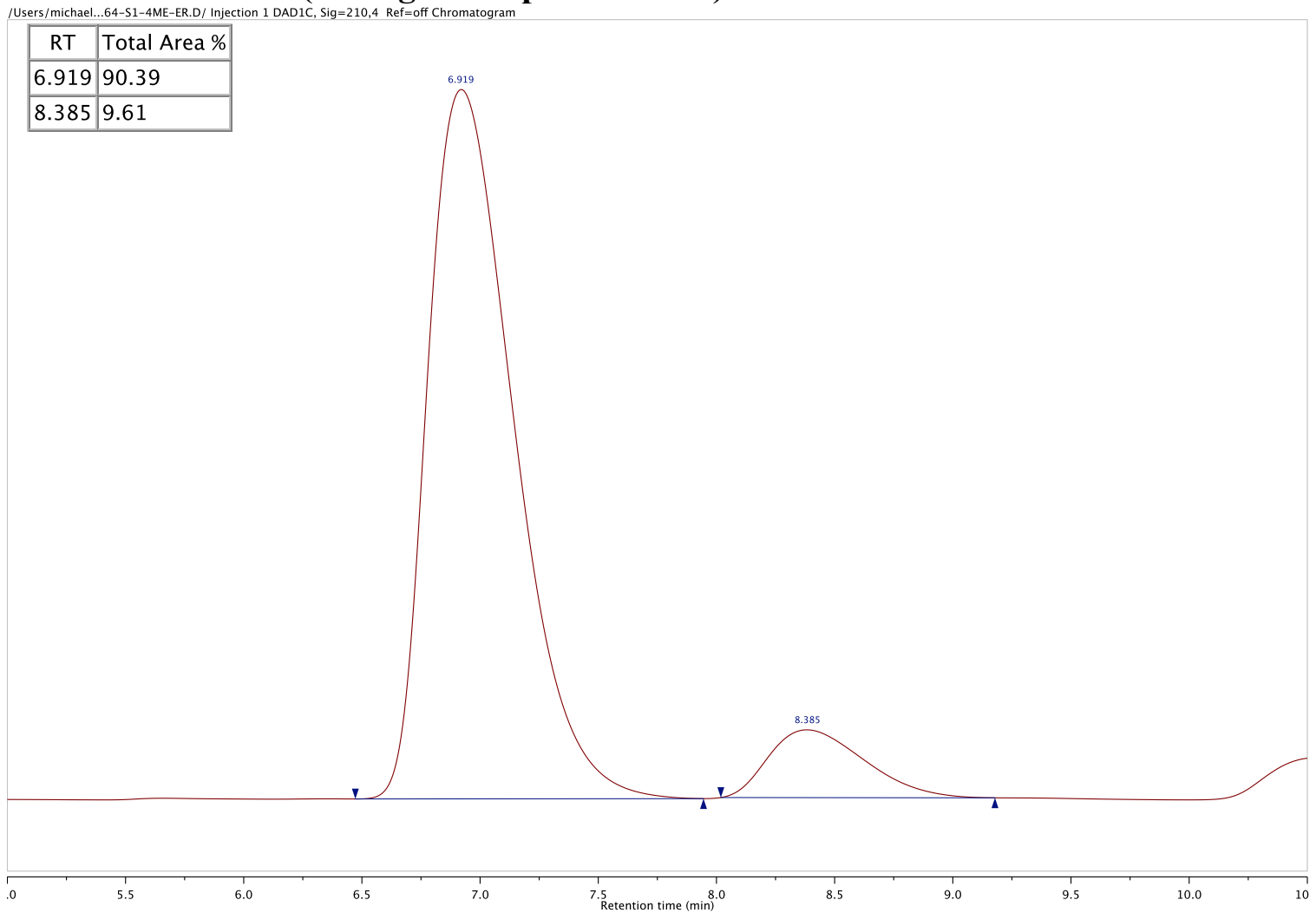




\section{Racemic 7}

/Users/michael...14-34OME-RAC.D/ Injection 1 DAD1C, Sig=210,4 Ref=off Chromatogram

\begin{tabular}{|c|l|}
\hline RT & Total Area \% \\
\hline 24.503 & 48.46 \\
\hline 28.569 & 51.54 \\
\hline
\end{tabular}

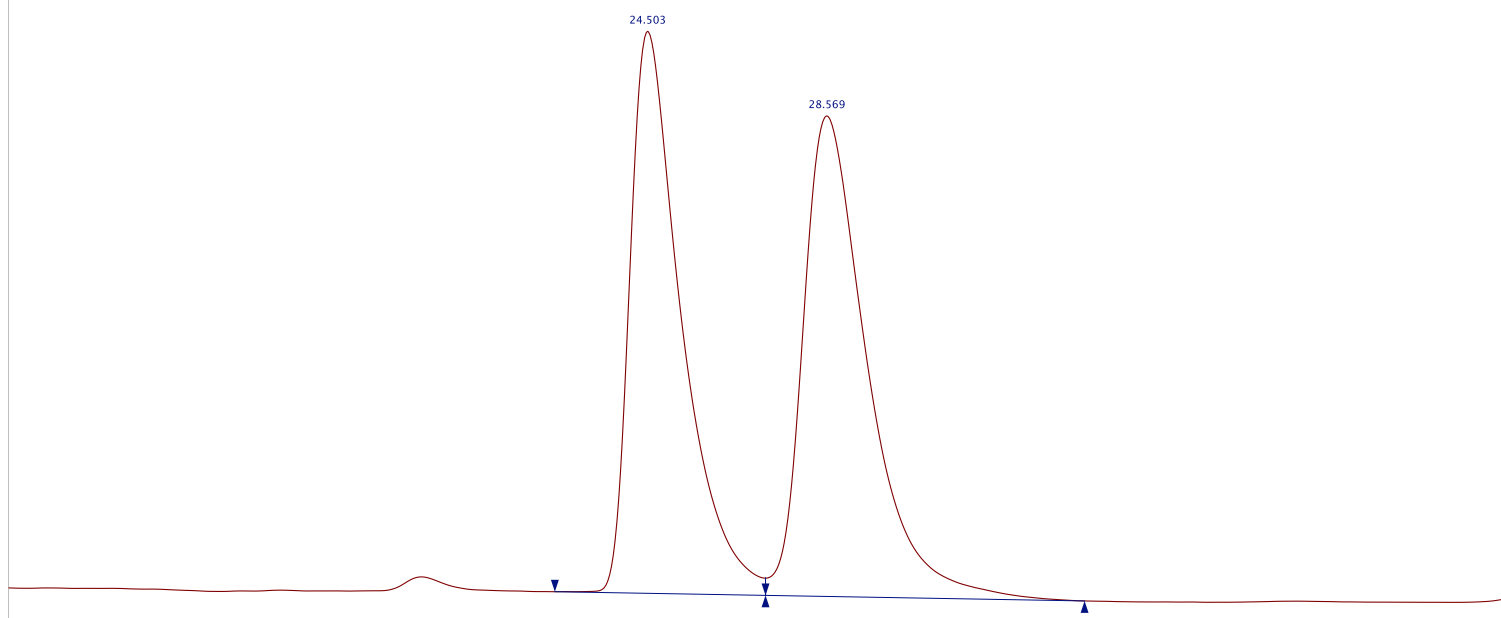

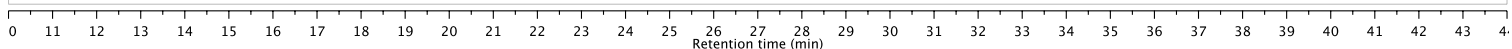
Enantioenriched 7 (from general procedure B)

/Users/michael...-S1-340ME-ER.D/ Injection
\begin{tabular}{|c|l|}
\hline RT & Total Area \% \\
\hline 24.290 & 9.87 \\
\hline 28.356 & 90.13 \\
\hline
\end{tabular}

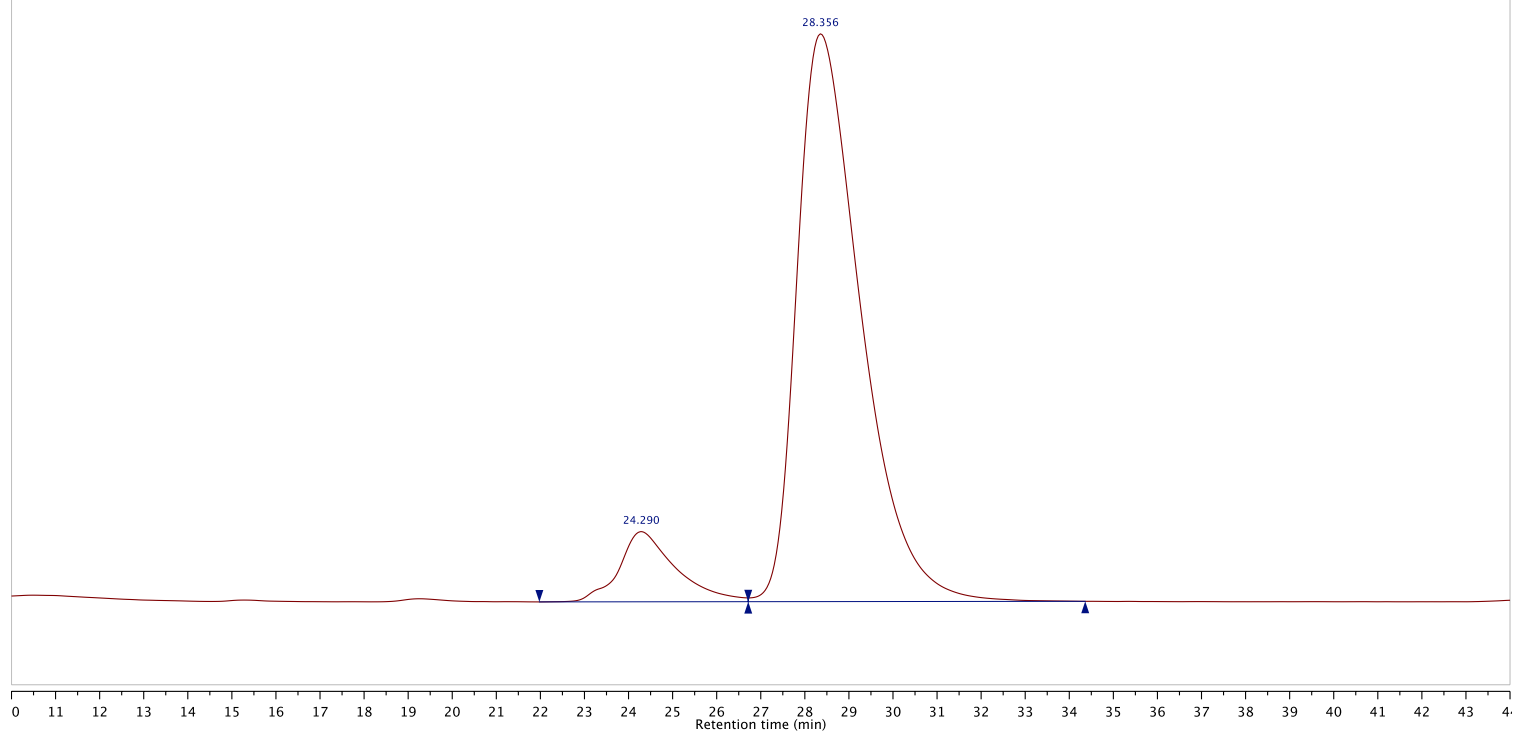




\section{Racemic 9}

/Users/michael...-PLUG-3F-RAC.D/ Injection 1 DAD1C, Sig=210,4 Ref=off Chromatogram

\begin{tabular}{|c|l|}
\hline RT & Total Area \% \\
\hline 8.155 & 50.08 \\
\hline 17.546 & 49.92 \\
\hline
\end{tabular}

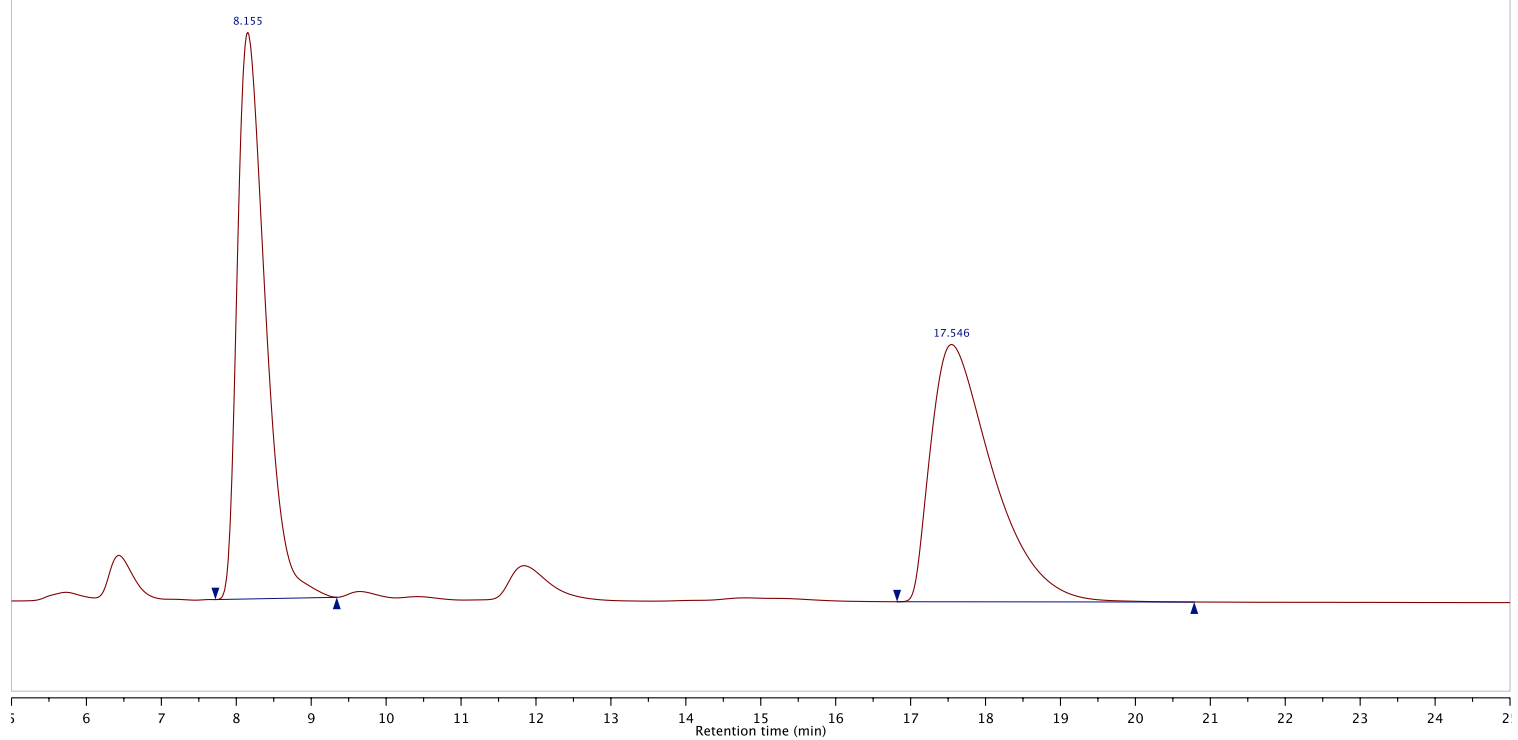

Enantioenriched 9 (from general procedure B)

/Users/michael...-39-S1-3F-ER.D/ Injectio
\begin{tabular}{|c|l|}
\hline RT & Total Area \% \\
\hline 8.119 & 91.98 \\
\hline 17.384 & 8.02 \\
\hline
\end{tabular}

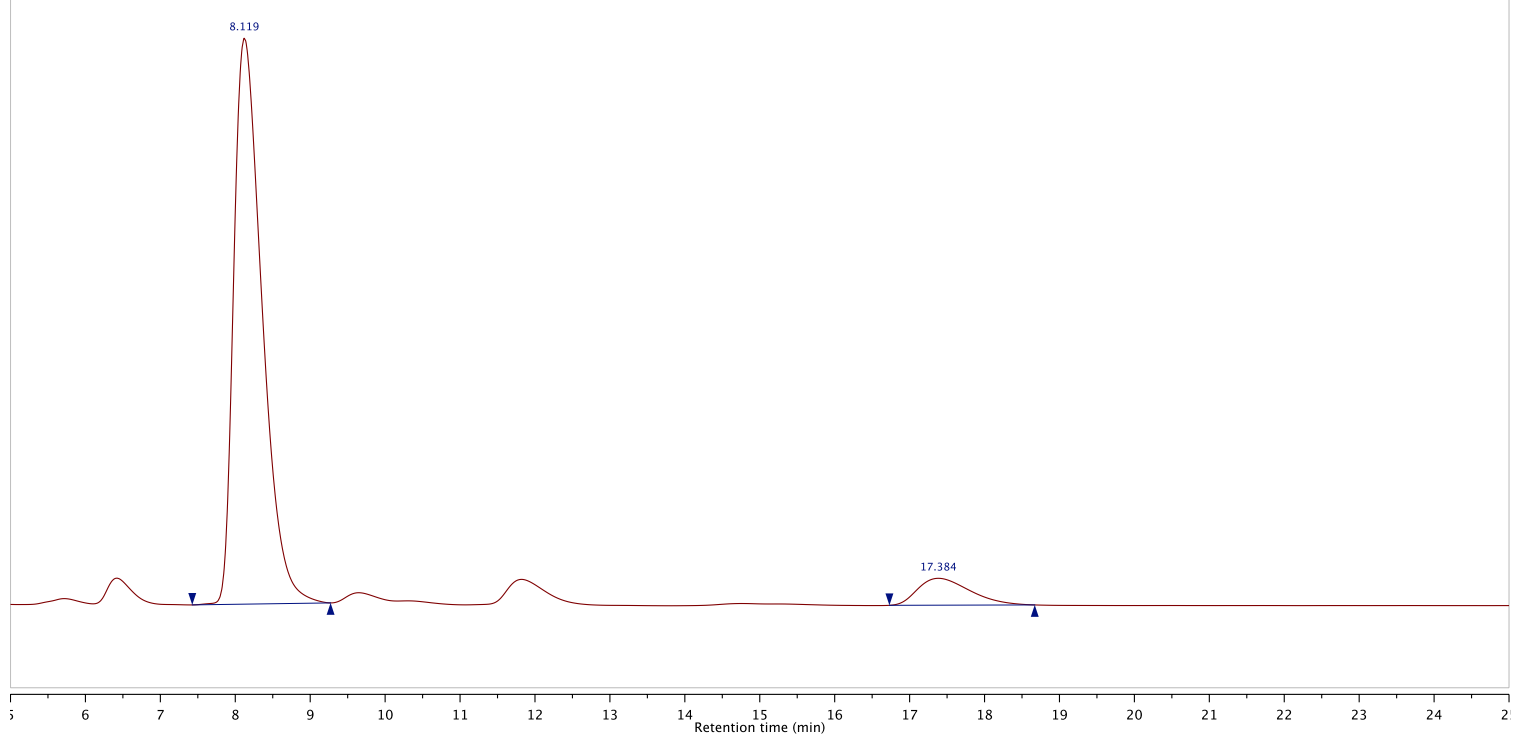




\section{Racemic 10}

IUsers/michael...18-44-2F-RAC.D/Injection 1 DADIC, Sig=210,4 Ref=off Chromatogram

\begin{tabular}{|c|l|}
\hline \multicolumn{1}{|c|}{ RT } & Total Area \% \\
\hline 8.839 & 49.75 \\
\hline 23.416 & 50.25 \\
\hline
\end{tabular}

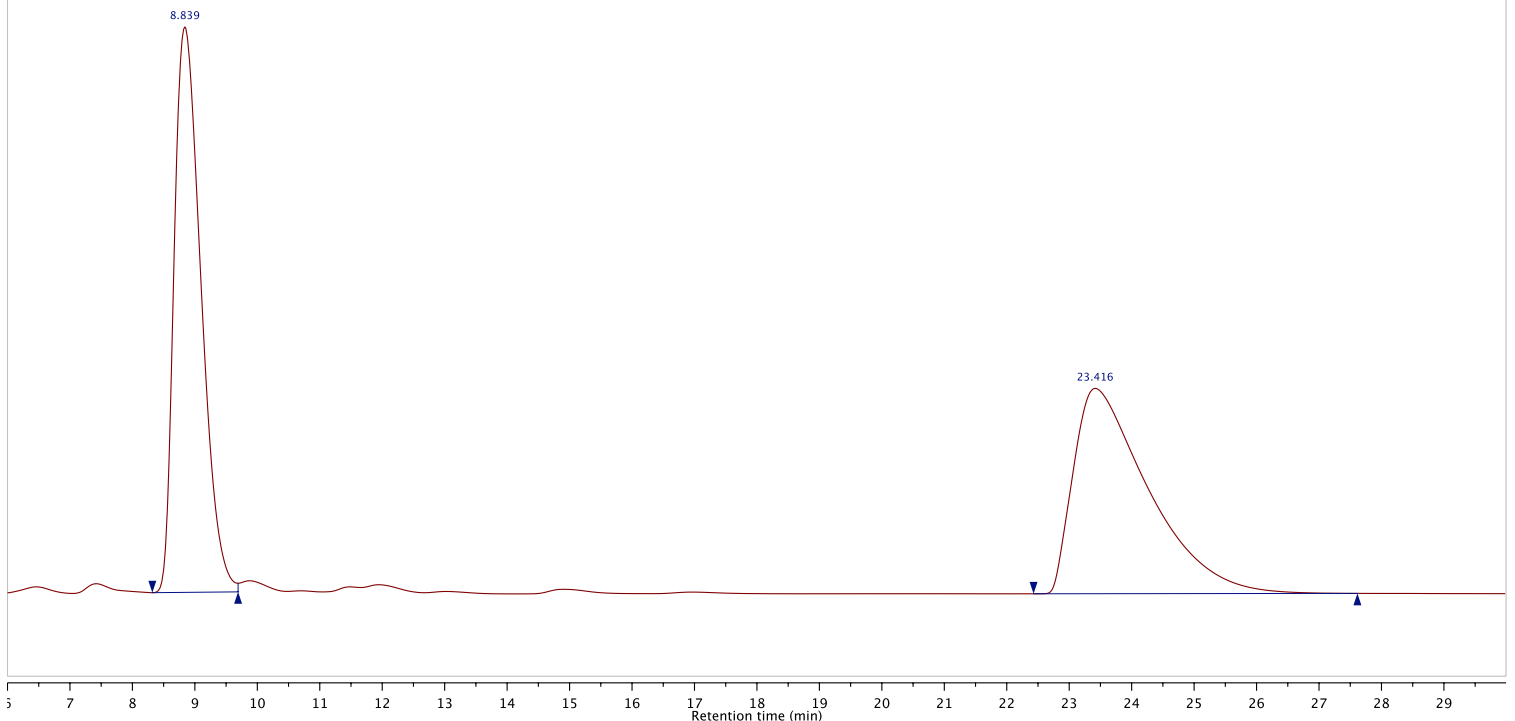

Enantioenriched 10 (from general procedure B)

\section{/Users/michael...-68-S1-2F-ER.D/ Injection 1 DAD1C, Sig=210,4 Ref=off Chromatogram}

\begin{tabular}{|c|l|}
\hline RT & Total Area \% \\
\hline 24.121 & 12.65 \\
\hline 8.823 & 87.35 \\
\hline
\end{tabular}

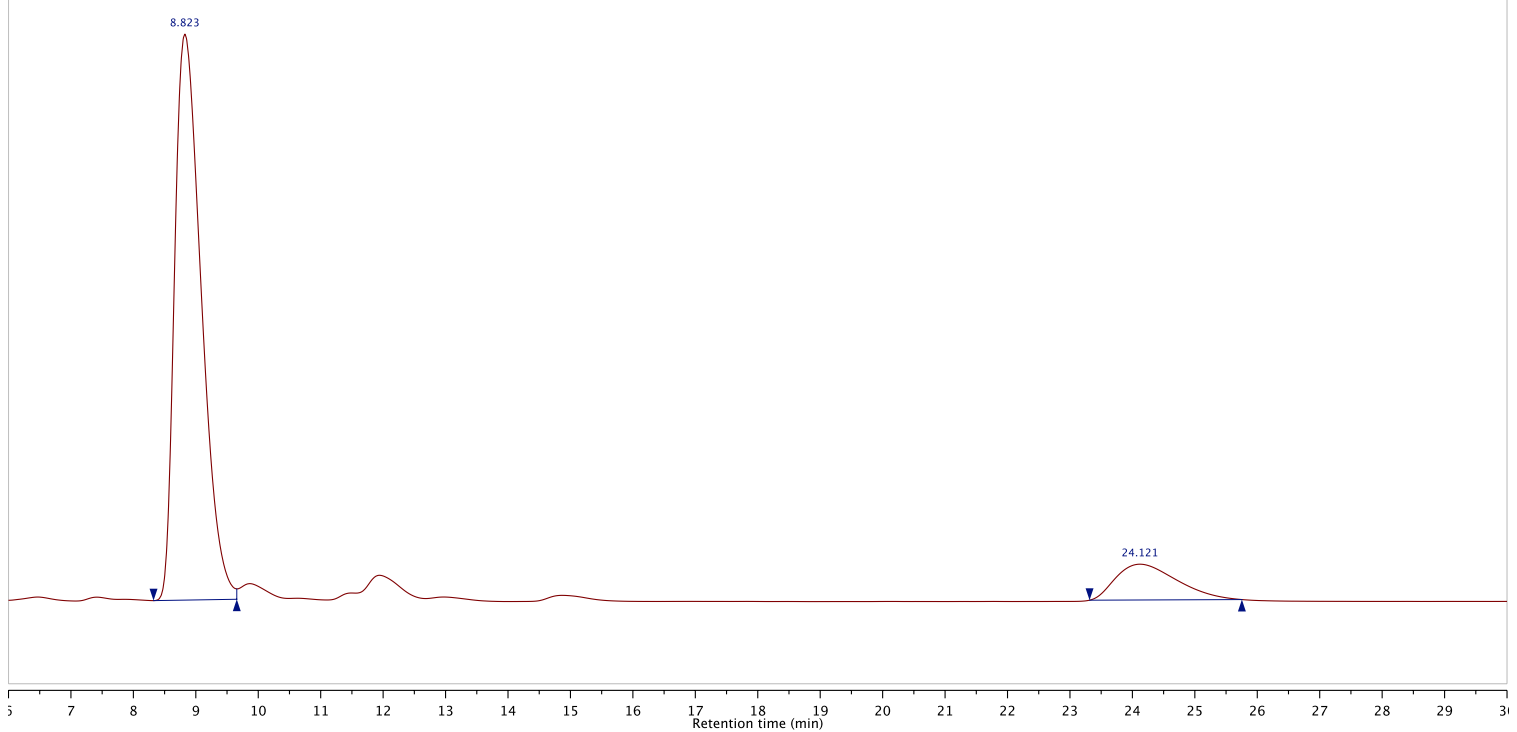




\section{Racemic 12}

/Users/michael...-45-3NO2-RAC.D/ Injection 1 DAD1C, Sig=210,4 Ref=off Chromatogram

\begin{tabular}{|c|l|}
\hline RT & Total Area \% \\
\hline 12.217 & 51.12 \\
\hline 24.548 & 48.88 \\
\hline
\end{tabular}
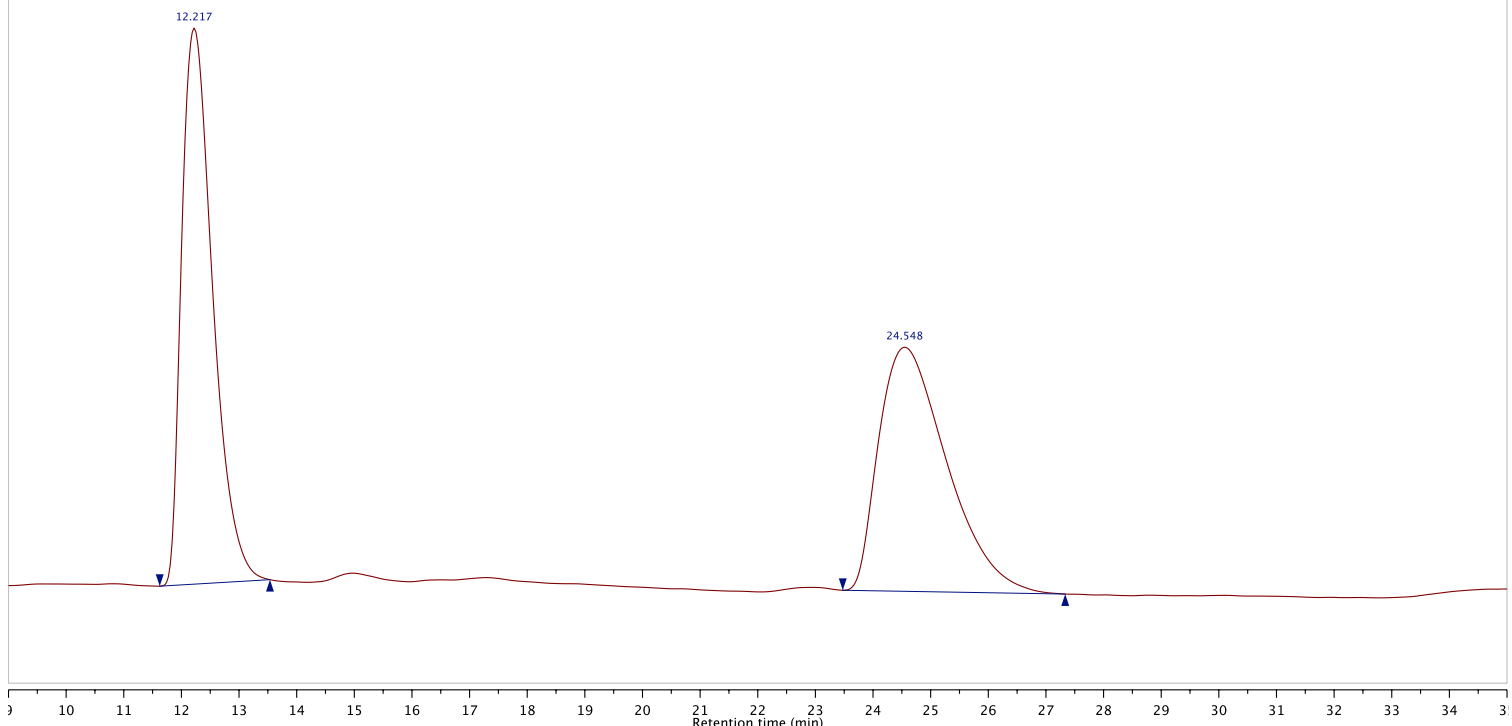

\section{Enantioenriched 12 (from general procedure B) \\ /Users/michael...5-S1-3N02-ER.D/ Injection 1 DAD1C, Sig=210,4 Ref=off Chromatogram}

\begin{tabular}{|c|l|}
\hline RT & Total Area \% \\
\hline 24.766 & 17.21 \\
\hline 12.394 & 82.79 \\
\hline
\end{tabular}

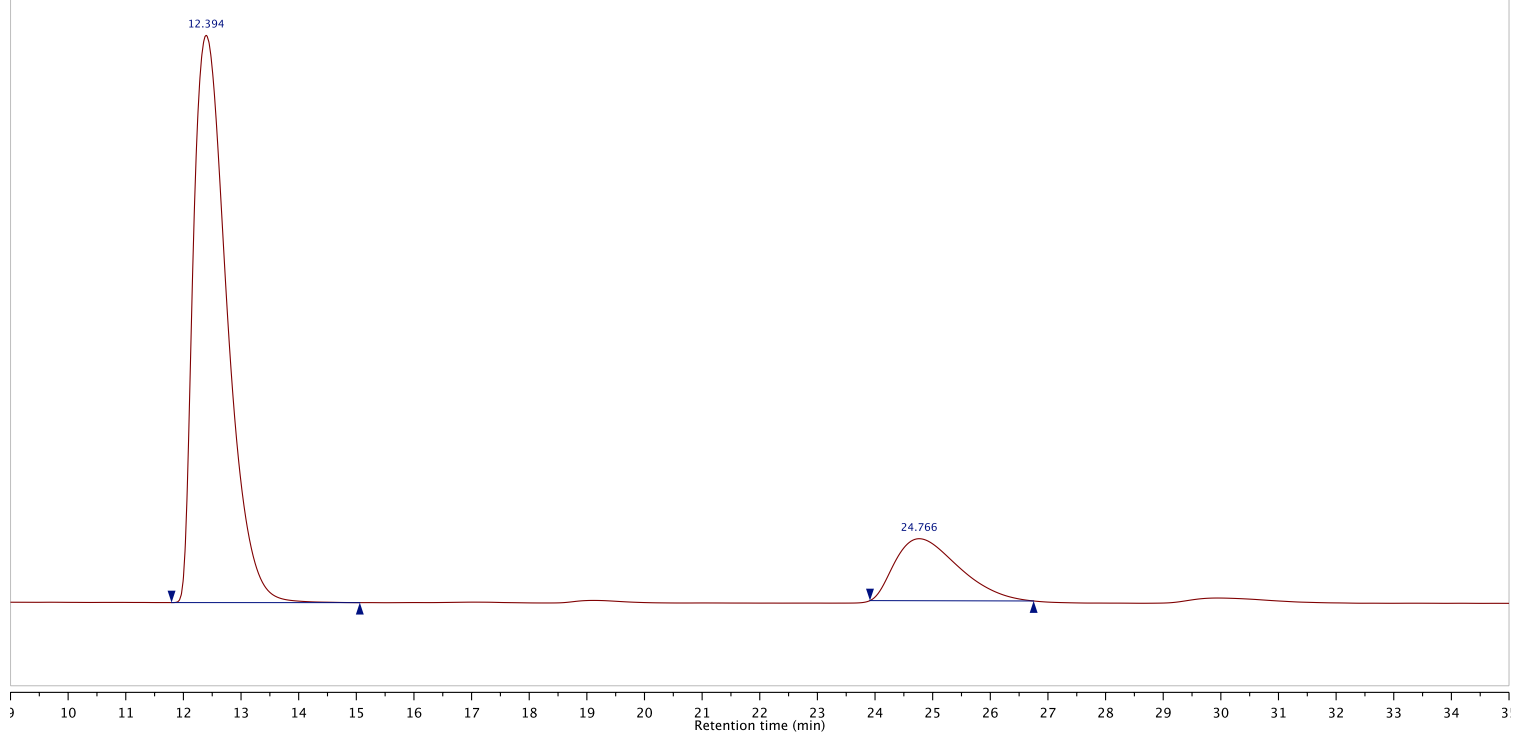




\section{Racemic 13}

/Users/michael...-46-THIO-RAC.D/ Injection 1 DAD1D, Sig=230,4 Ref=off Chromatogram

\begin{tabular}{|c|l|}
\hline RT & Total Area \% \\
\hline 15.193 & 49.95 \\
\hline 36.943 & 50.05 \\
\hline
\end{tabular}

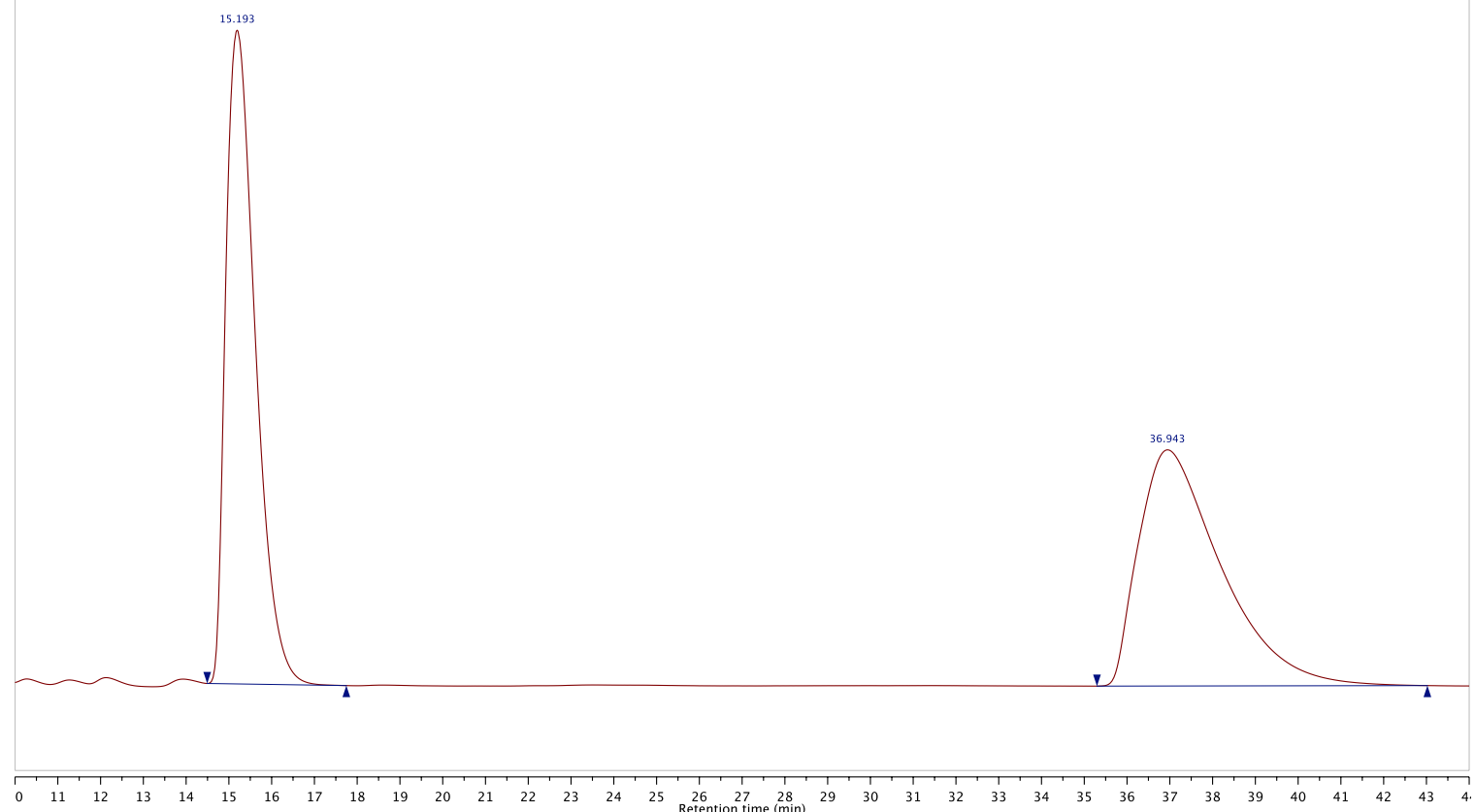

Enantioenriched 13 (from general procedure B)

\section{/Users/michael.,6-S1-THIO-ER.D/Injection 1 DAD1D, Sig $=230,4$ Ref $=$ off Chromatogram}

\begin{tabular}{|c|c|c|}
\hline RT & Total Area \% \\
\hline
\end{tabular}

29.57523 .09

\begin{tabular}{l|l|l|}
\hline 13.550 & 76.91
\end{tabular}

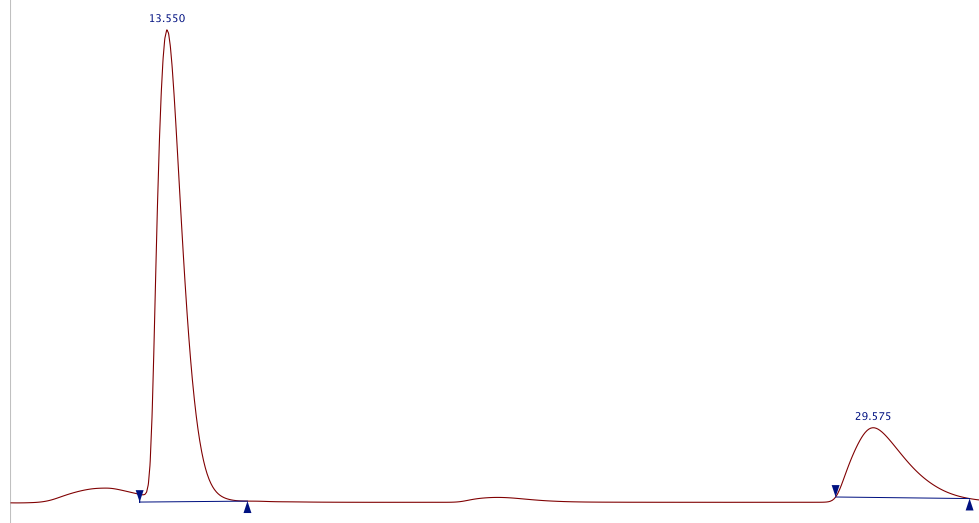

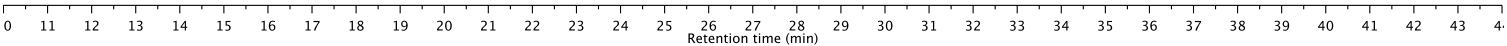




\section{Racemic 14}

/Users/michael...44-S1-BN-RAC.D/ Injection 1 DAD1C, Sig=210,4 Ref=off Chromatogram

\begin{tabular}{|c|l|}
\hline RT & Total Area \% \\
\hline 12.699 & 50.96 \\
\hline 18.098 & 49.04 \\
\hline
\end{tabular}

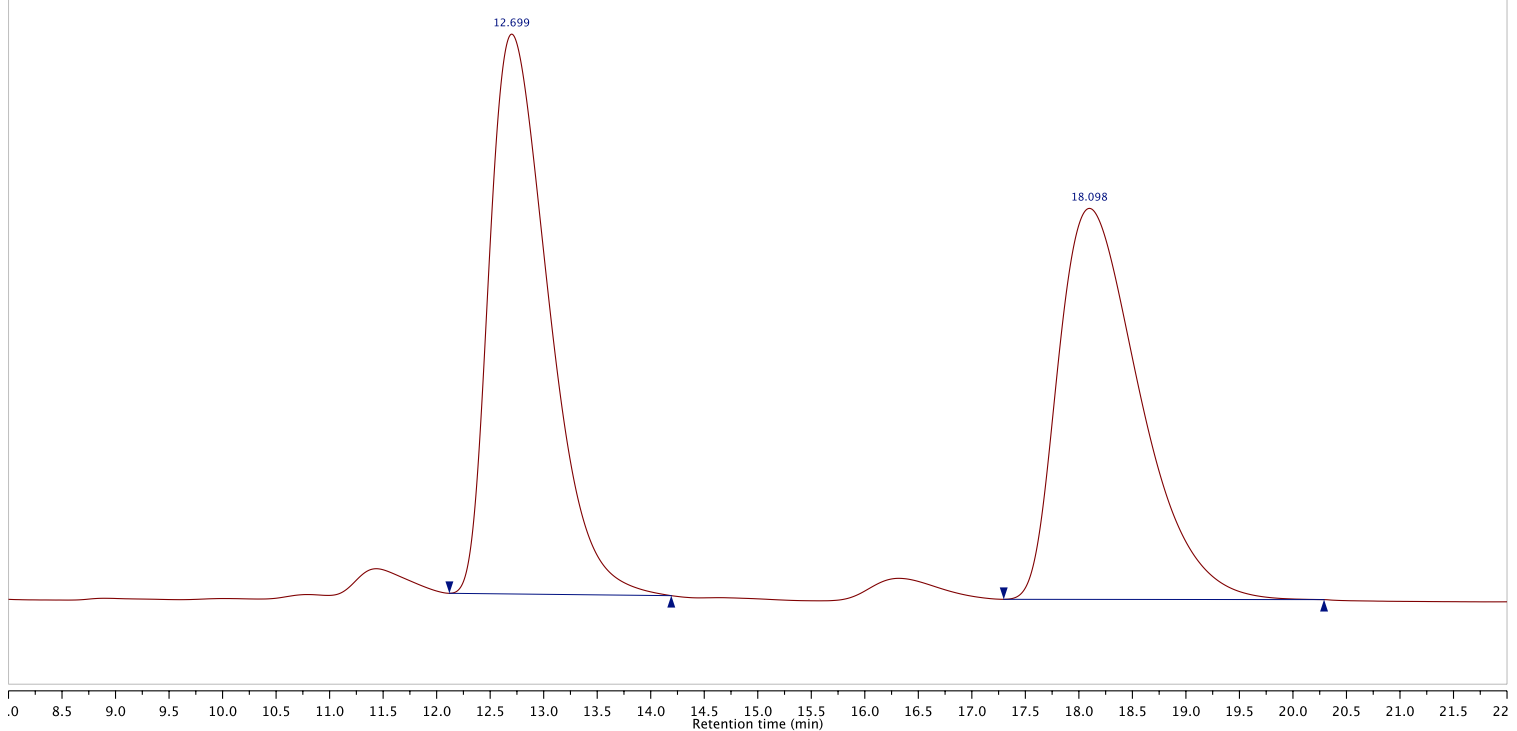

Enantioenriched 14 (from general procedure B)

\begin{tabular}{|c|l|} 
/Users/michael...-25-42-BN-ER.D/ Injectio \\
\hline RT & Total Area \% \\
\hline 12.617 & 84.74 \\
\hline 18.022 & 15.26 \\
\hline
\end{tabular}

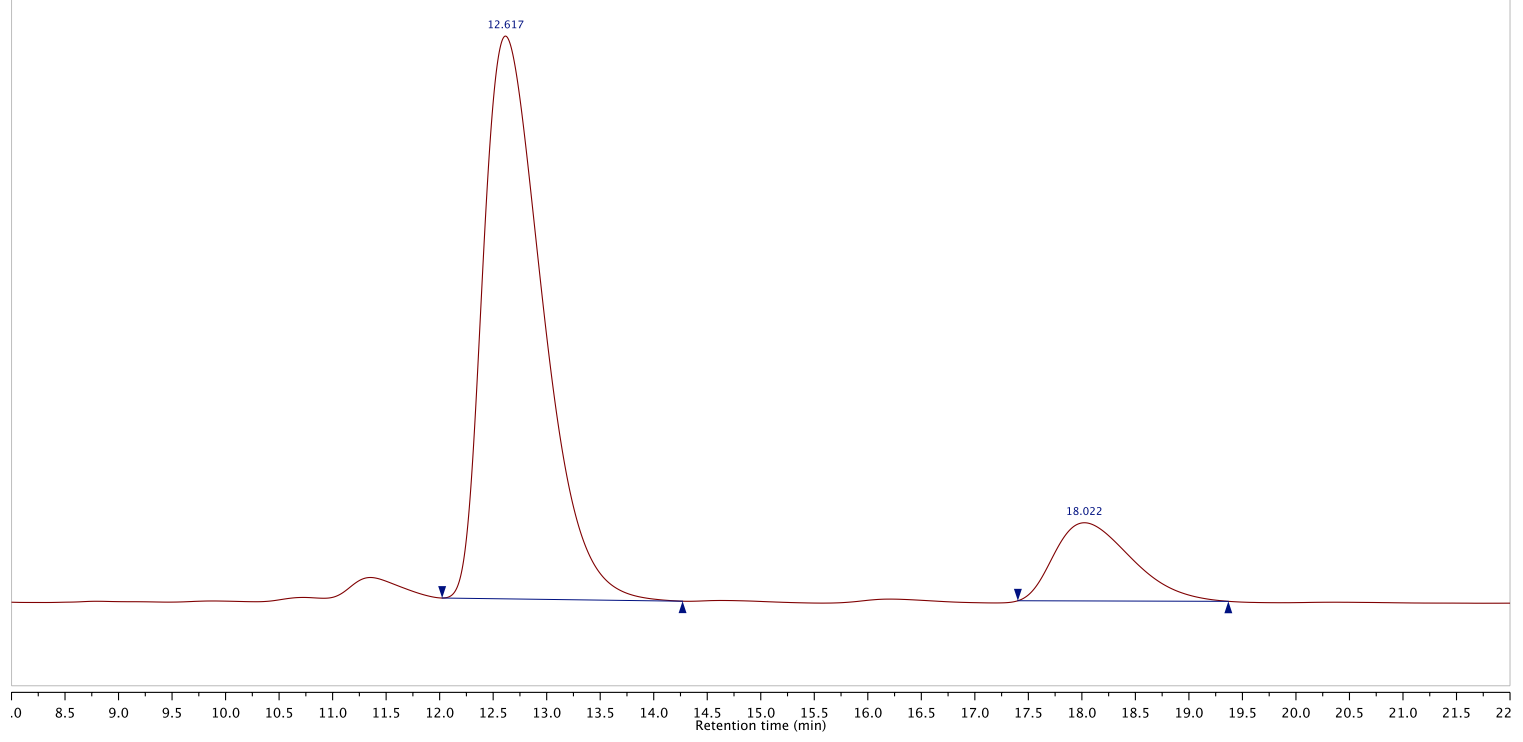




\section{Racemic 22}

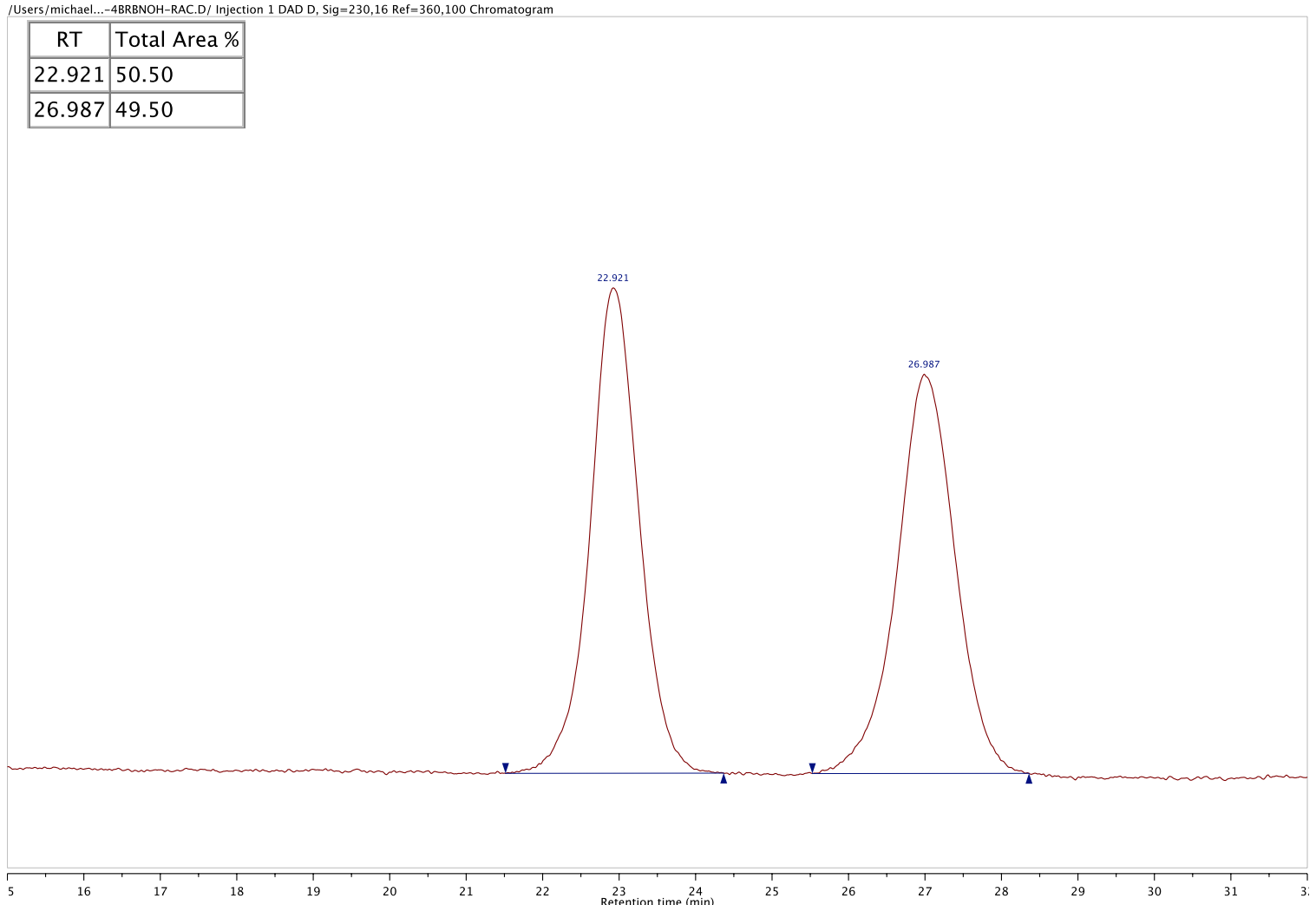

Recrystallized 22

/Users/michael...9-LOWERXTALS.D/ Injection 1 DAD D, Sig=230,16 Ref=360,100 Chromatogram.

\begin{tabular}{|c|l|}
\hline RT & Total Area \% \\
\hline
\end{tabular}

\begin{tabular}{l|l|l}
\hline 26.522 & 98.56
\end{tabular}

\begin{tabular}{l|l|l|l}
22.769 & 1.44
\end{tabular}

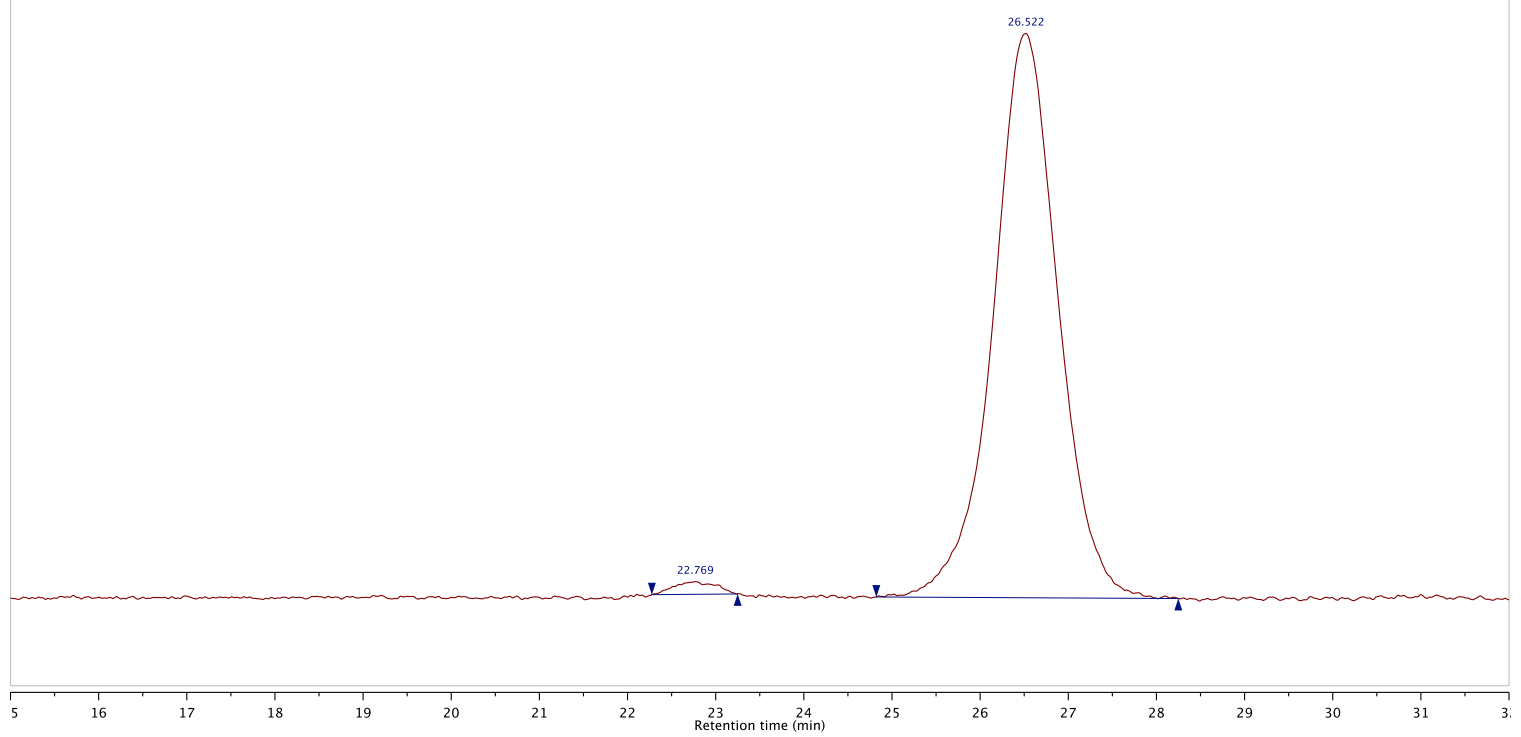




\section{Racemic 25}

/Users/michael...-18-92-4-LAC.D/Injection 1 DAD1C, Sig=210,4 Ref=off Chromatogram \begin{tabular}{c|c} 
RT & Total Area \%
\end{tabular}

36.10750 .04

40.61949 .96

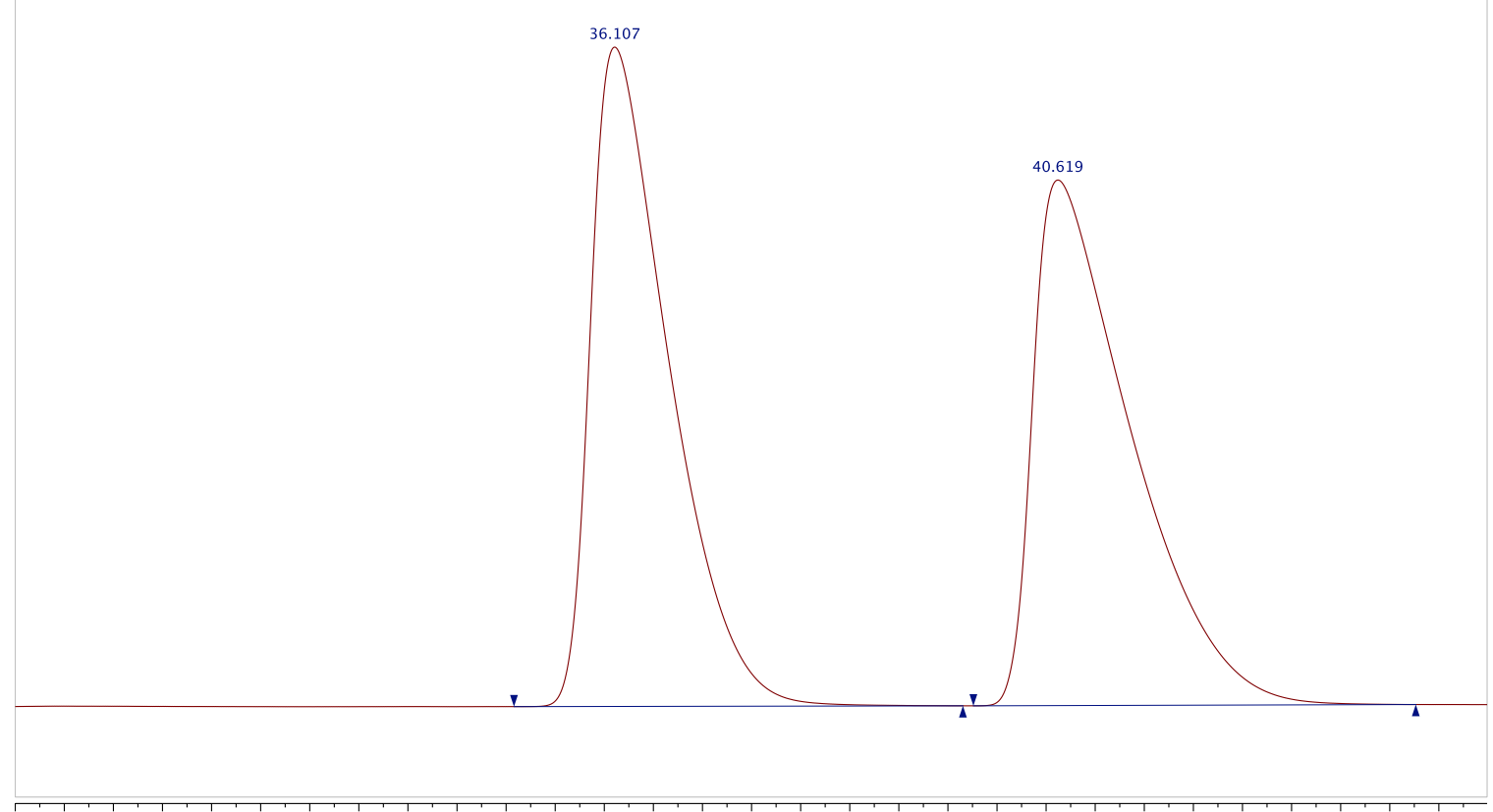

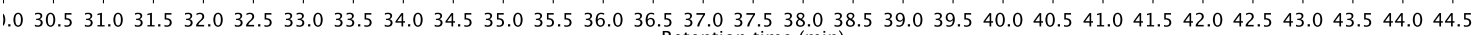

\section{Enantioenriched 25}

/Users/michael.../MW-23-71-S1.D/ Injection 1 DAD1C, Sig=210,4 Ref=off Chromatogram

\begin{tabular}{|c|l|}
\hline RT & Total Area \% \\
\hline 36.239 & 93.76 \\
\hline 42.071 & 6.24 \\
\hline
\end{tabular}

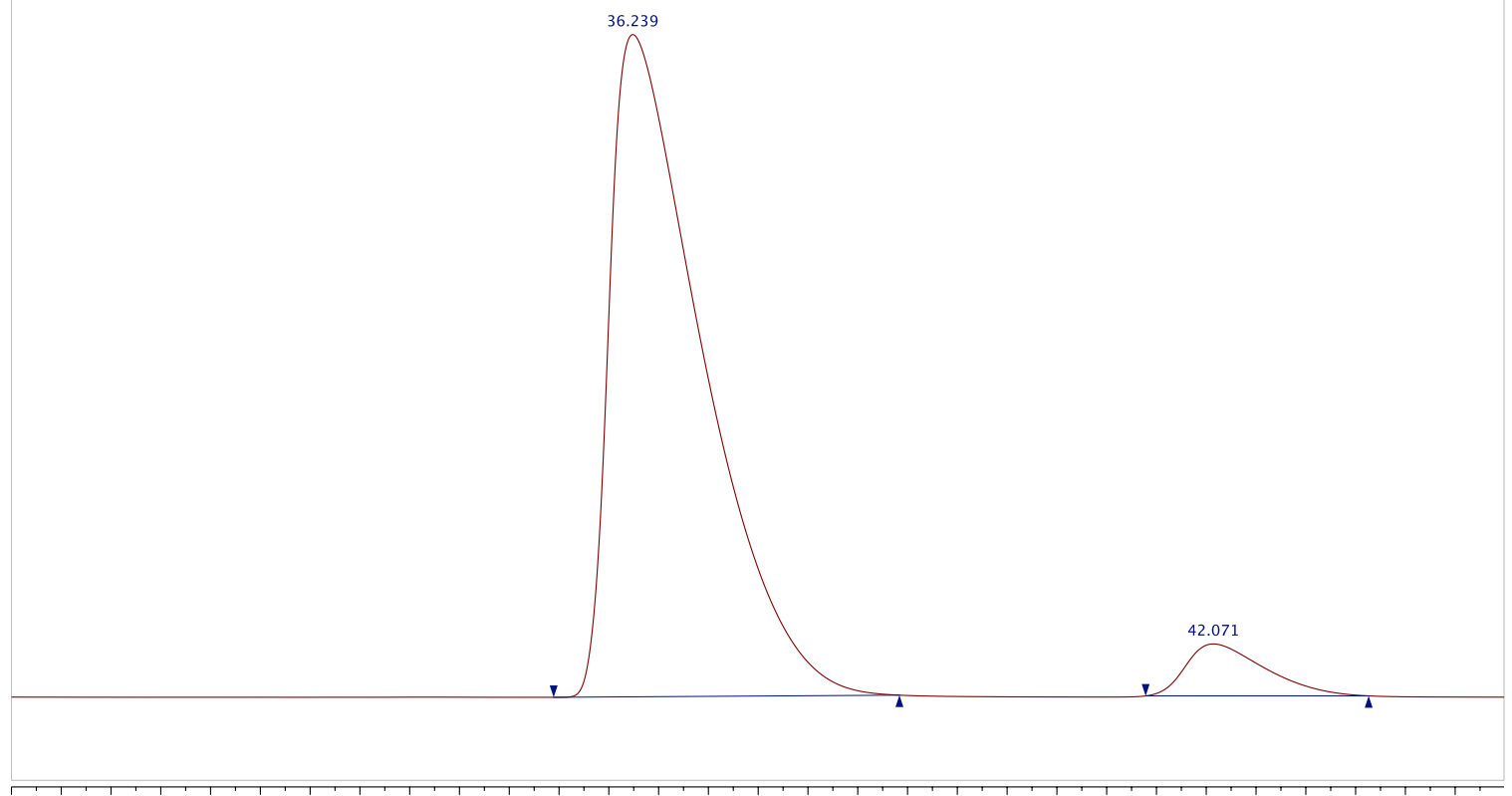

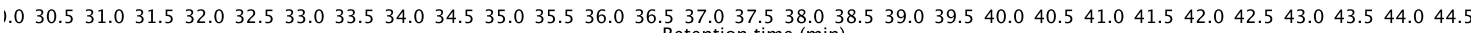
Retention time ( $\mathrm{min})$ 


\section{Racemic 26}

/Users/michael...-18-84-3-LAC.D/Injection 1 DAD1C, Sig=210,4 Ref=off Chromatogram

\begin{tabular}{|c|c|c|}
\hline RT & Total Area \% \\
\hline
\end{tabular}

25.53850 .13

28.41849 .87

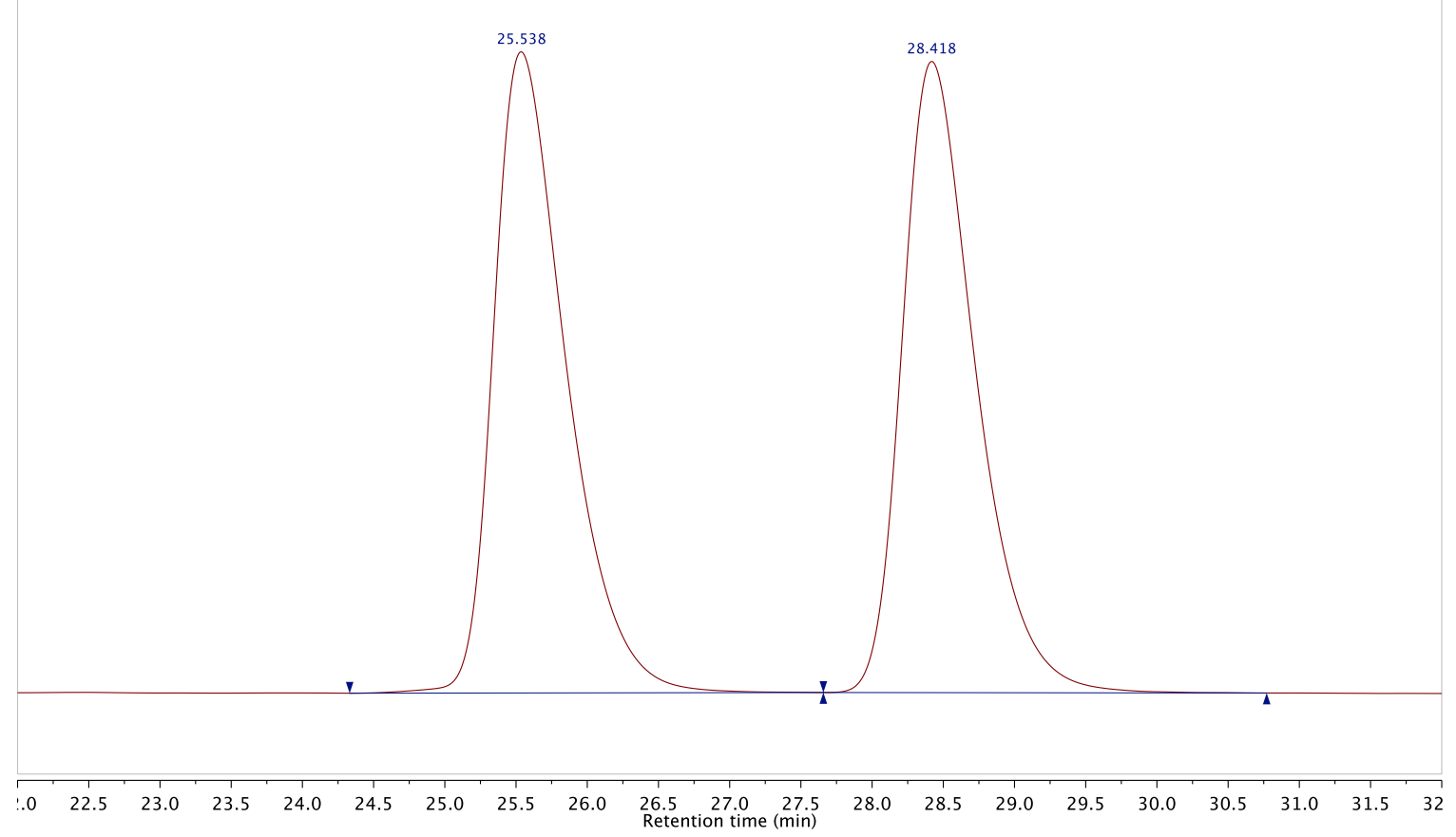

Enantioenriched 26

/Users/michael...W-28-36-S1-3.D/ Injection 1 DAD1C, Sig=210,4 Ref=off Chromatogram

\begin{tabular}{|c|l|}
\hline RT & Total Area \% \\
\hline 25.530 & 9.37 \\
\hline 28.163 & 90.63 \\
\hline
\end{tabular}

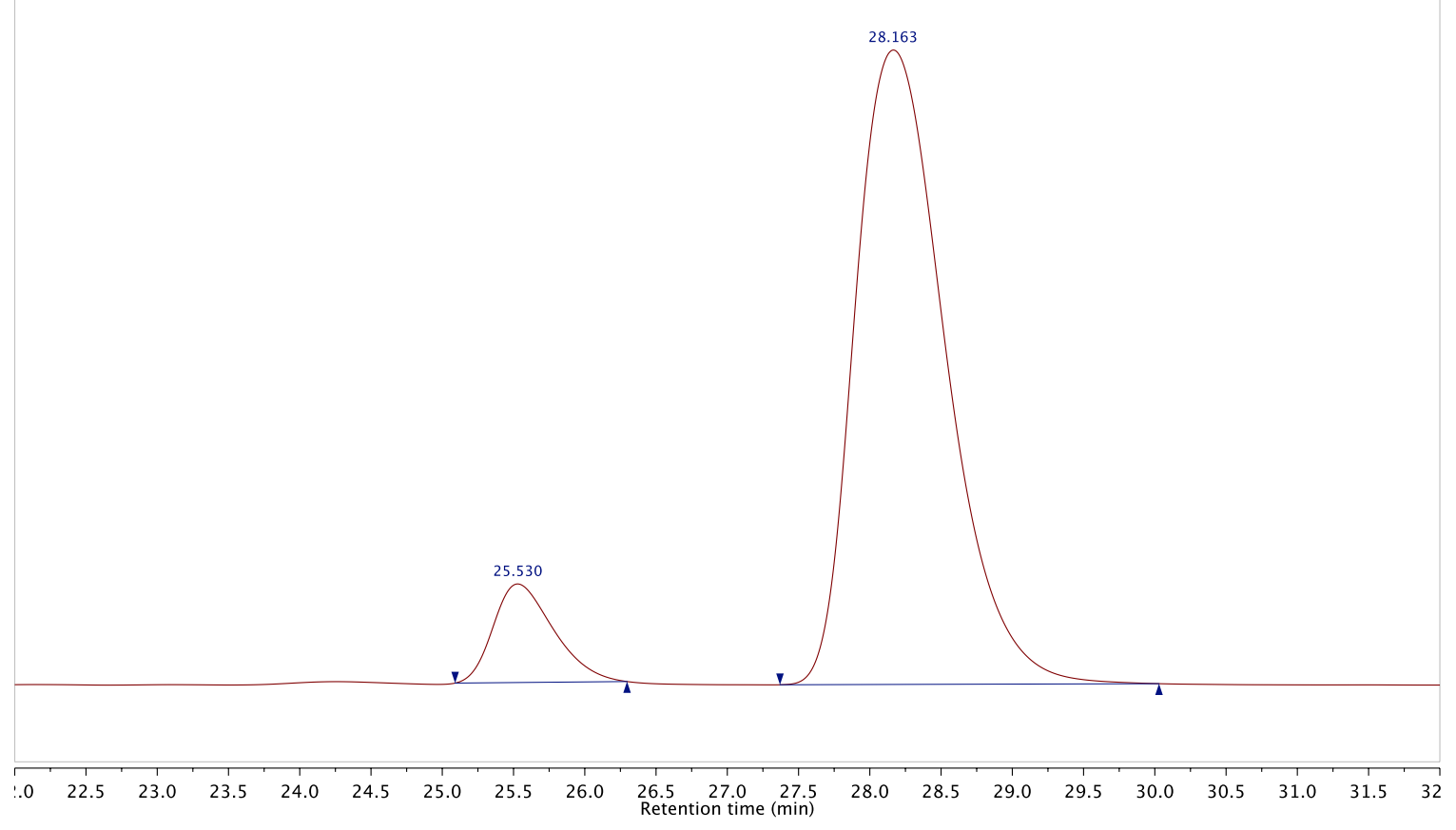




\section{Racemic 27}

I/Users/michael...C-18-86-B-AE.D/ Injection 1 DAD1B, Sig=254,4 Ref=off Chromatogram

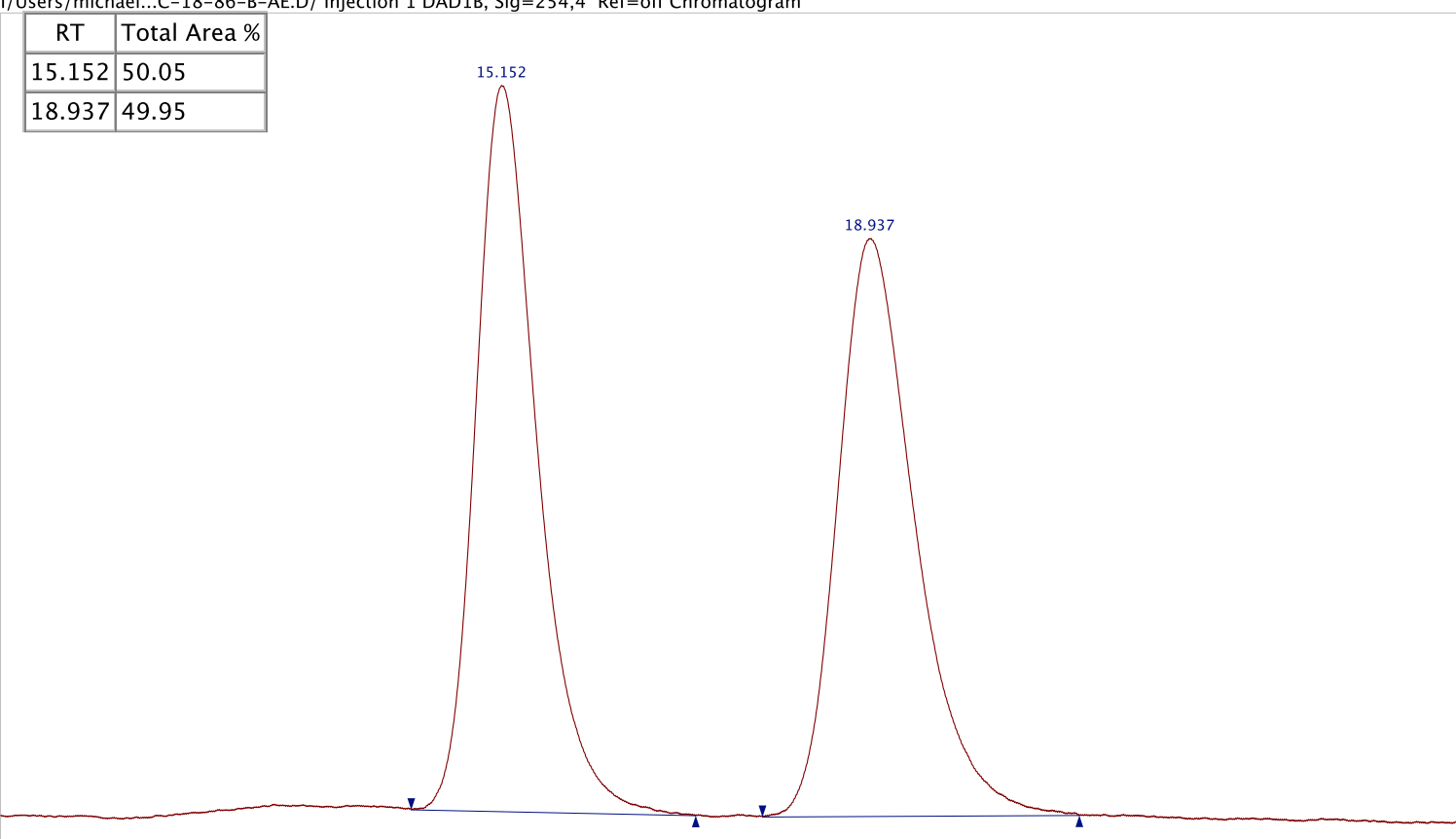

1.010 .511 .011 .512 .012 .513 .013 .514 .014 .515 .015 .516 .016 .517 .017 .518 .018 .519 .019 .520 .020 .521 .021 .522 .022 .523 .023 .524 .024 .525

\section{Enantioenriched 27}

love/Users/michael.../MW-28-39-S1.D/ Injection 1 DAD1B, Sig=254,4 Ref=off Chromatogram

\begin{tabular}{|c|l|}
\hline RT & Total Area \% \\
\hline 15.362 & 5.85 \\
\hline 18.334 & 94.15 \\
\hline
\end{tabular}

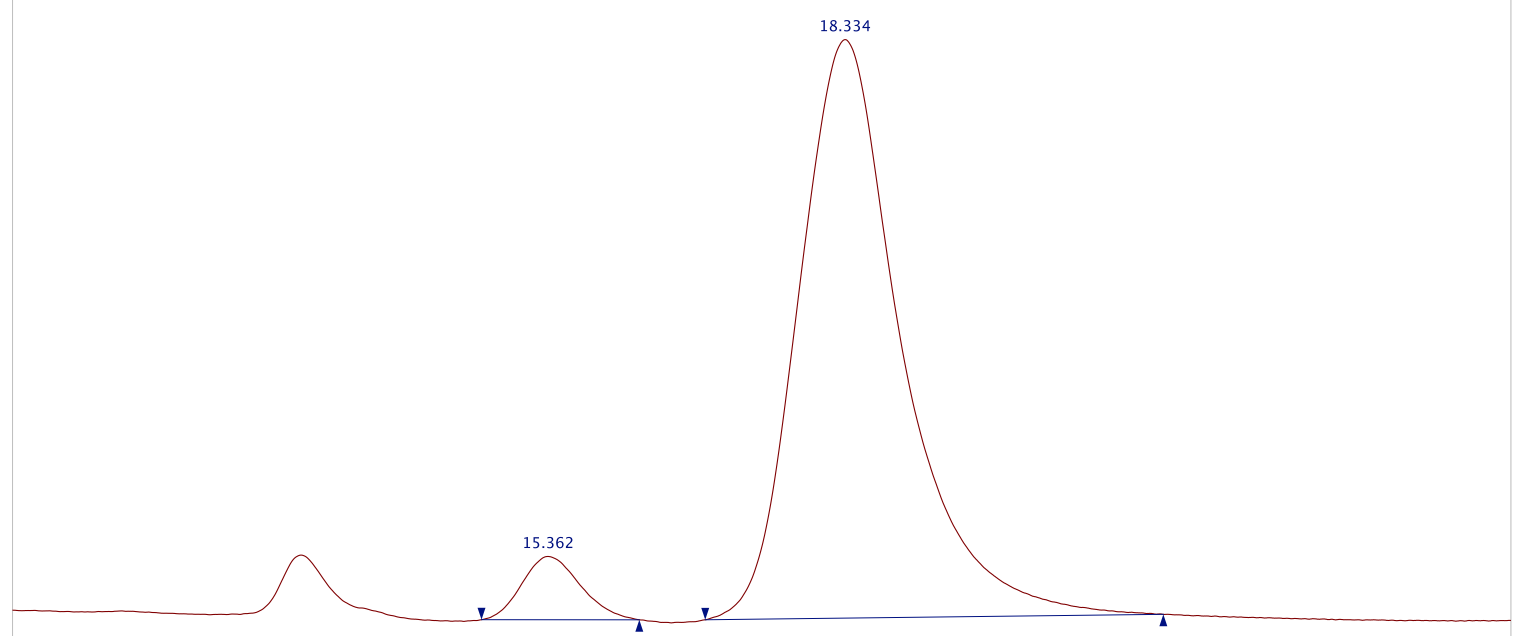

$\begin{array}{lllllllllllllllllllllllllllllllllllllllllllll}1.0 & 10.5 & 11.0 & 11.5 & 12.0 & 12.5 & 13.0 & 13.5 & 14.0 & 14.5 & 15.0 & 15.5 & 16.0 & 16.5 & 17.0 & 17.5 & 18.0 & 18.5 & 19.0 & 19.5 & 20.0 & 20.5 & 21.0 & 21.5 & 22.0 & 22.5 & 23.0 & 23.5 & 24.0 & 24.5 & 25\end{array}$ Retention time ( $\mathrm{min})$ 


\section{Racemic 28}

you/Users/michael...18-72-A-AE-3.D/ Injection 1 DAD1C, Sig=210,4 Ref=off Chromatogram \begin{tabular}{c|c} 
RT & Total Area \% \\
\hline
\end{tabular}

38.77050 .07

45.69049 .93

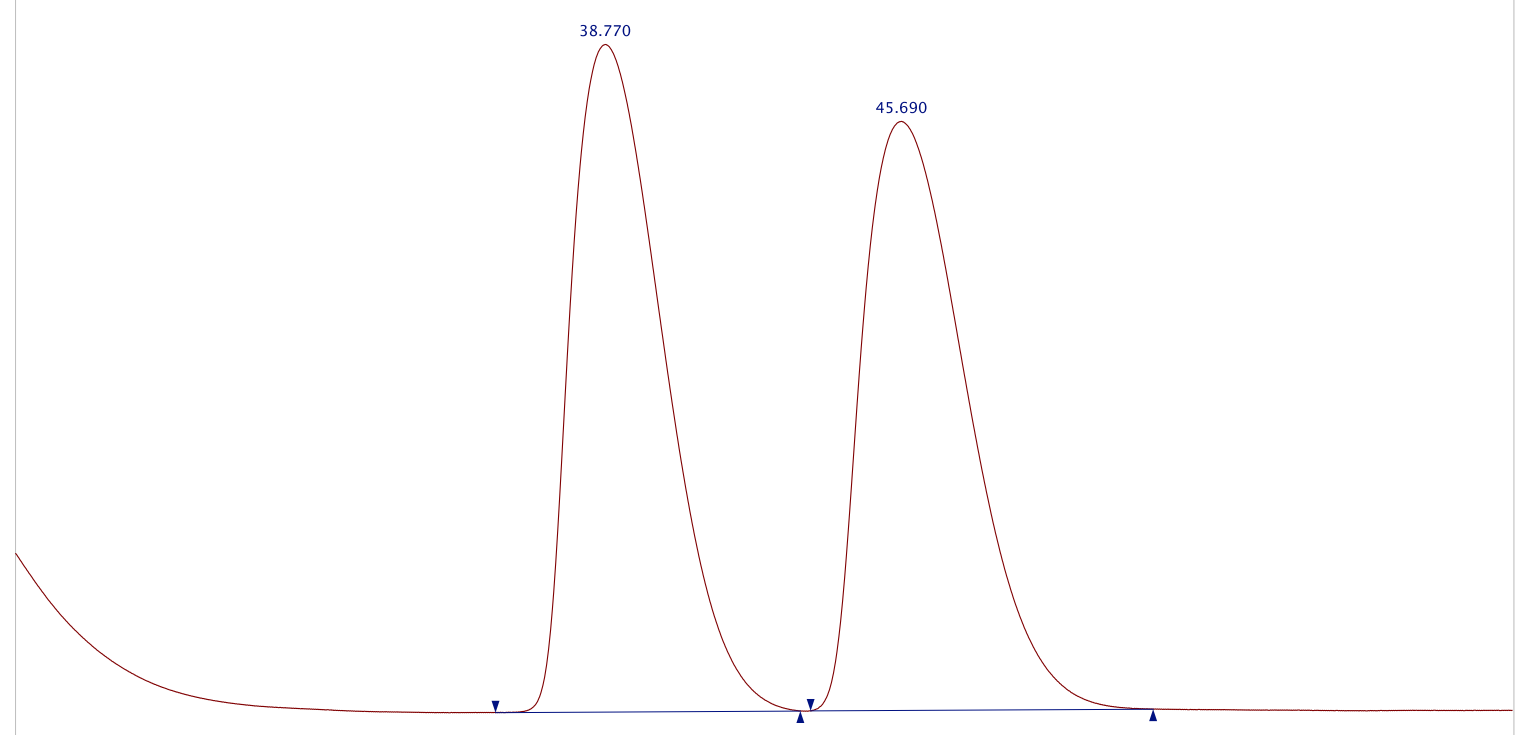

$\begin{array}{lllllllllllllllllllllllllllllllllllll}5 & 26 & 27 & 28 & 29 & 30 & 31 & 32 & 33 & 34 & 35 & 36 & 37 & 38 & 39 & 40 & 41 & 42 & 43 & 44 & 45 & 46 & 47 & 48 & 49 & 50 & 51 & 52 & 53 & 54 & 55 & 56 & 57 & 58 & 59\end{array}$

\section{Enantioenriched 28}

LiebeBeiqin/Users/michael.../MW-23-72-S1.D/Injection 1 DAD1C, Sig=210,4 Ref=off Chromatogram

\begin{tabular}{|c|l|}
\hline RT & Total Area \% \\
\hline 39.675 & 7.41 \\
\hline 45.954 & 92.59 \\
\hline
\end{tabular}

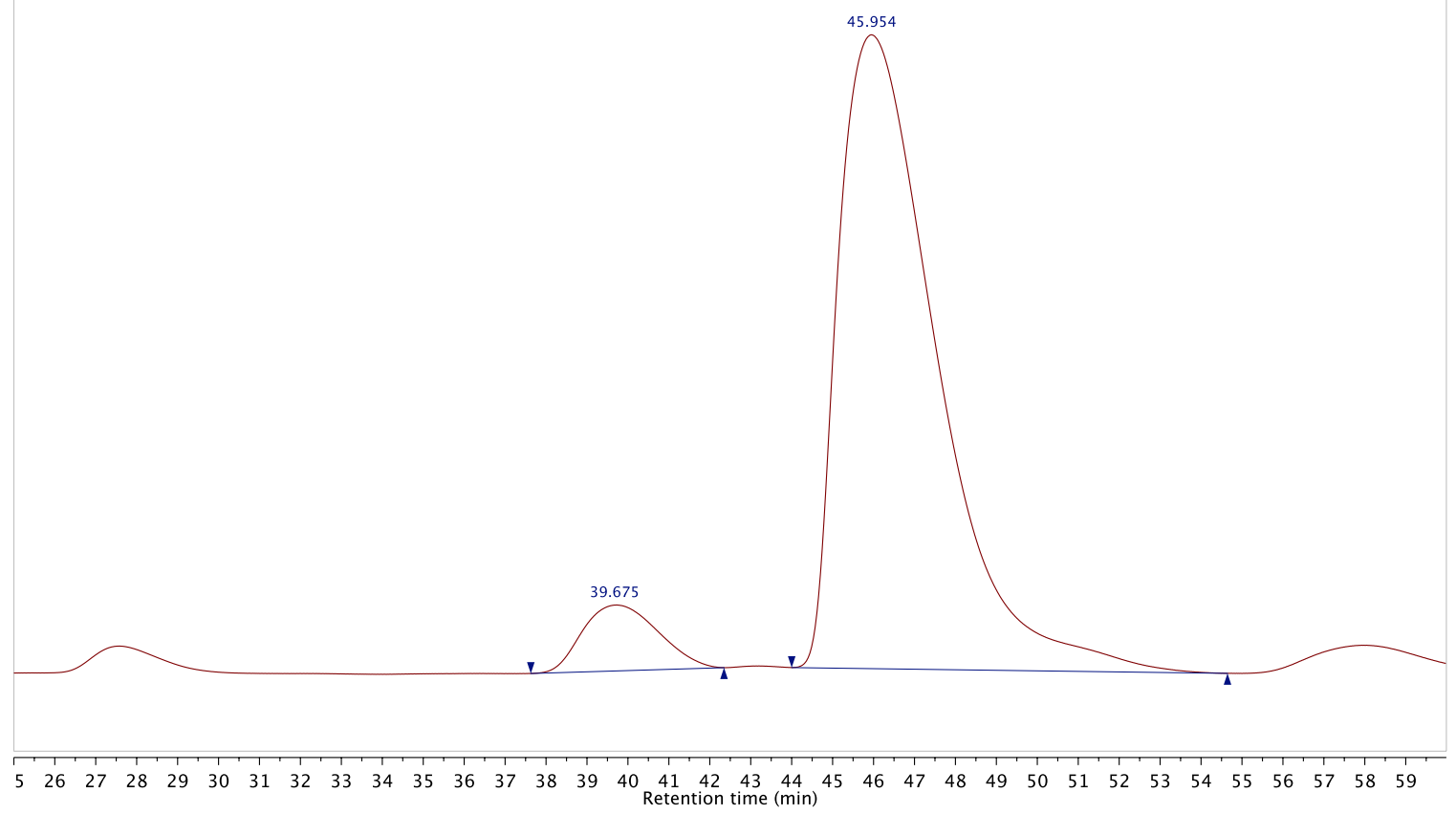




\section{Control experiment with $Z$-isomer starting material}

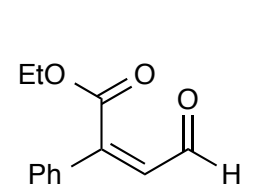

$1-Z$
$10 \mathrm{~mol} \% \mathrm{~A}$ $30 \mathrm{~mol} \%$ HBD3 $40 \mathrm{~mol} \%$ i-Pr${ }_{2} \mathrm{NEt}$

10 equiv $\mathrm{EtOH}$

DCE, $0^{\circ} \mathrm{C}, 12 \mathrm{~h}$

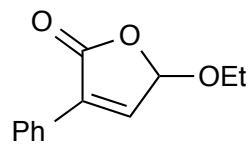

SI-1

5-ethoxy-3-phenylfuran-2(5H)-one (SI-1): Prepared according to the general procedure using (Z)-ethyl 4-oxo-2-phenylbut-2-enoate $(0.041 \mathrm{~g}, 0.200 \mathrm{mmol})$ and purified by flash chromatography using $8 \%$ EtOAc/hexanes to afford $0.0372 \mathrm{mg}$ (91\% yield) of SI-1 as a colorless oil. Analytical data for SI-1: ${ }^{1} \mathrm{H}$ NMR $\left(500 \mathrm{MHz}, \mathrm{CDCl}_{3}\right) \delta 7.84(\mathrm{dd}, \mathrm{J}=6.6$, $3.1 \mathrm{~Hz}, 2 \mathrm{H}), 7.42(\mathrm{dd}, \mathrm{J}=5.0,1.8 \mathrm{~Hz}, 3 \mathrm{H}), 7.28(\mathrm{~d}, \mathrm{~J}=1.3 \mathrm{~Hz}, 1 \mathrm{H}), 5.95(\mathrm{~d}, \mathrm{~J}=1.3 \mathrm{~Hz}$, $1 \mathrm{H}), 4.05-3.93(\mathrm{~m}, 1 \mathrm{H}), 3.87-3.75(\mathrm{~m}, 1 \mathrm{H}), 1.32(\mathrm{t}, \mathrm{J}=7.1 \mathrm{~Hz}, 3 \mathrm{H}) ;{ }^{13} \mathrm{C}$ NMR $(126$ $\left.\mathrm{MHz}, \mathrm{CDCl}_{3}\right) \delta 169.8,141.5,135.2,130.1,129.0,128.8,127.6,100.8,66.2,15.3$; LRMS (ESI): Mass calcd for $\mathrm{C}_{12} \mathrm{H}_{13} \mathrm{O}_{3}[\mathrm{M}+\mathrm{H}]^{+}:$205; found 205 . 


\section{Procedure for DFT calculations}

The Density Functional Theory (DFT) calculations were performed on the compounds NHC_HE1 and NHC_HE2 using Jaguar 7.8 implemented in the Schrodinger 9.1 suite. For this closed shell system, we used the $6-31 \mathrm{G}^{* *++}$ basis set with a hybrid functional of B3LYP. Selecting a fine grid density option and applying a convergence scheme of DIIS the optimization of the compound was performed. The energy optimization was carried out along with the computations of the vibrational frequencies and other thermochemical properties.

Ref: Jaguar, version 7.8, Schrodinger, Inc., New York, 2014 


\section{Procedure for 1D NOE experiment}

In an NMR tube was combined 1 (2.3 mg), HBD1 (5 mg), and $0.5 \mathrm{~mL}$ toluene-d8. 1D NOESY was acquired with irradiation on $3.88 \mathrm{ppm}($ delta $=0.1 \mathrm{ppm})$, with 1024 scans and $800 \mathrm{~ms}$ mixing time.

${ }^{1}$ H NMR (toluene-d8) 1+HBD1<smiles>CCC(C)(C)OC(OC)/C(=C\C=O)c1ccccc1</smiles>

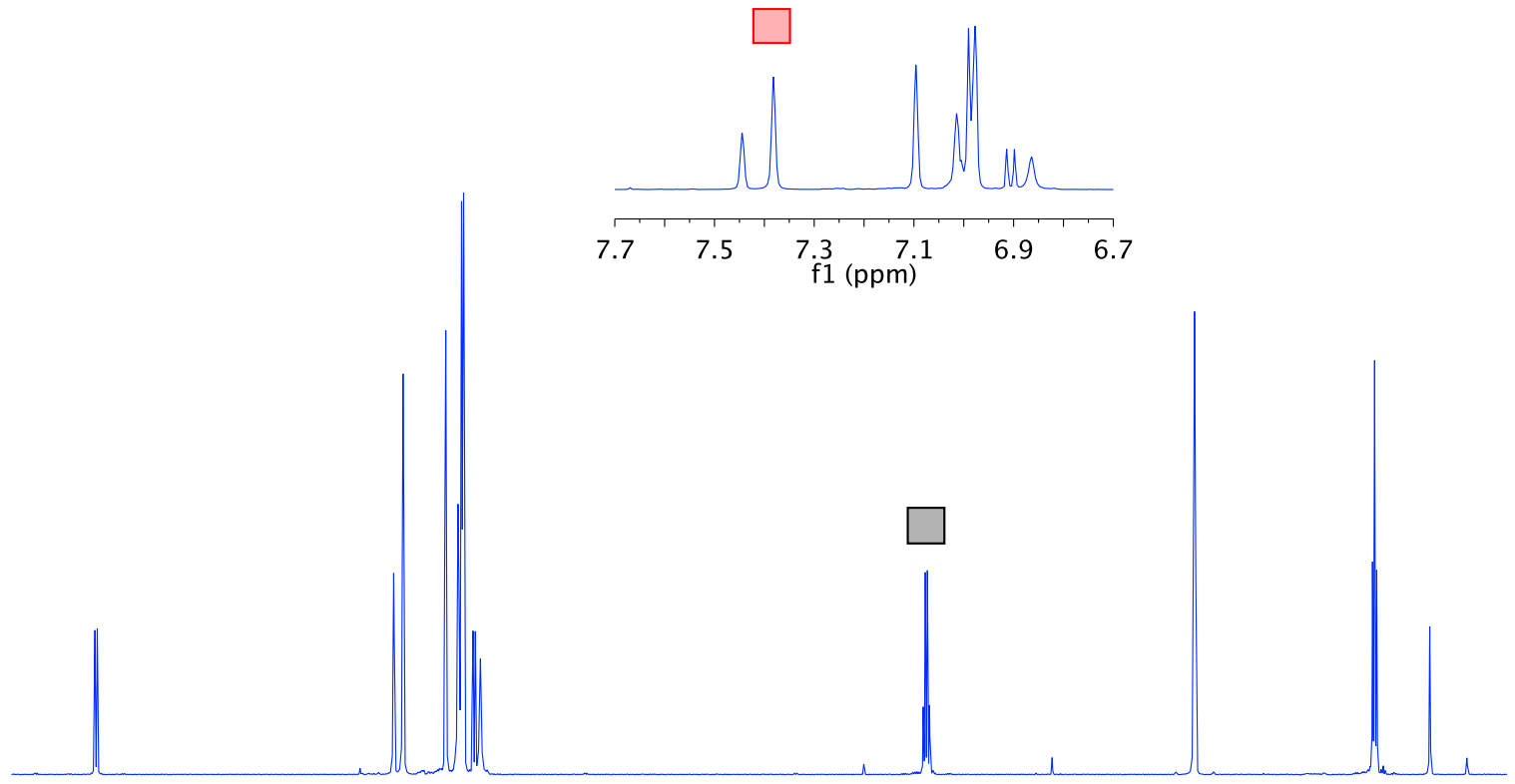

$\begin{array}{lllllllllllllllllllll}1.0 & 9.5 & 9.0 & 8.5 & 8.0 & 7.5 & 7.0 & 6.5 & 6.0 & 5.5 & \underset{f 1}{(\mathrm{ppm})} \mathbf{5 . 0} & 4.5 & 4.0 & 3.5 & 3.0 & 2.5 & 2.0 & 1.5 & 1.0 & 0.5 & 0 .\end{array}$ 


\section{D NOE (toluene-d8) 1+HBD1}
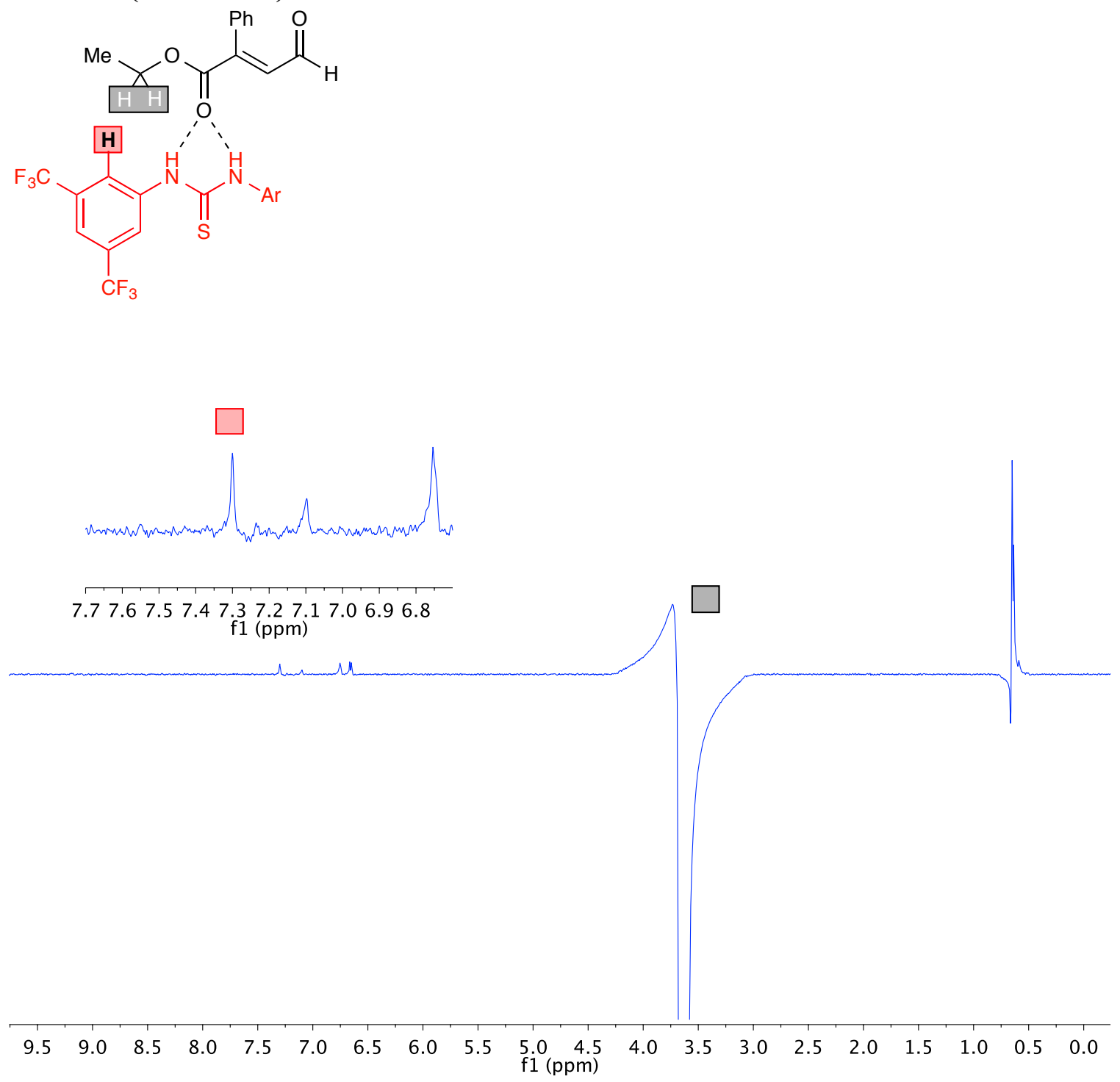


\section{Determination of Absolute Configuration of 22}

The absolute configuration of $\mathbf{2 2}$ was determined by X-ray diffraction. Recrystallized from hexanes.<smiles>CCOC(=O)C(CC(=O)OCc1ccc(Br)cc1)c1ccccc1</smiles>

X-ray crystal structure of 4-(4-bromobenzyl) 1-ethyl (S)-2-phenylsuccinate (22):

$\mathrm{X}$-ray diffraction was performed at $99.88 \mathrm{~K}$ and raw frame data were processed using SAINT. Molecular structures was solved using direct methods and refined on F2 by fullmatrix least-square techniques. The $\mathrm{GOF}=1.071$ for 218 variables refined to $\mathrm{R} 1=$ 0.0252 for 2803 reflections with $\mathrm{I}>2 \alpha(\mathrm{I})$. A multi-scan absorption correction was performed and the Flack parameter was -0.0033(10). Further information can be found in the CIF file. This crystal structure was deposited in the Cambridge Crystallographic Data Centre and assigned as 1037055 .

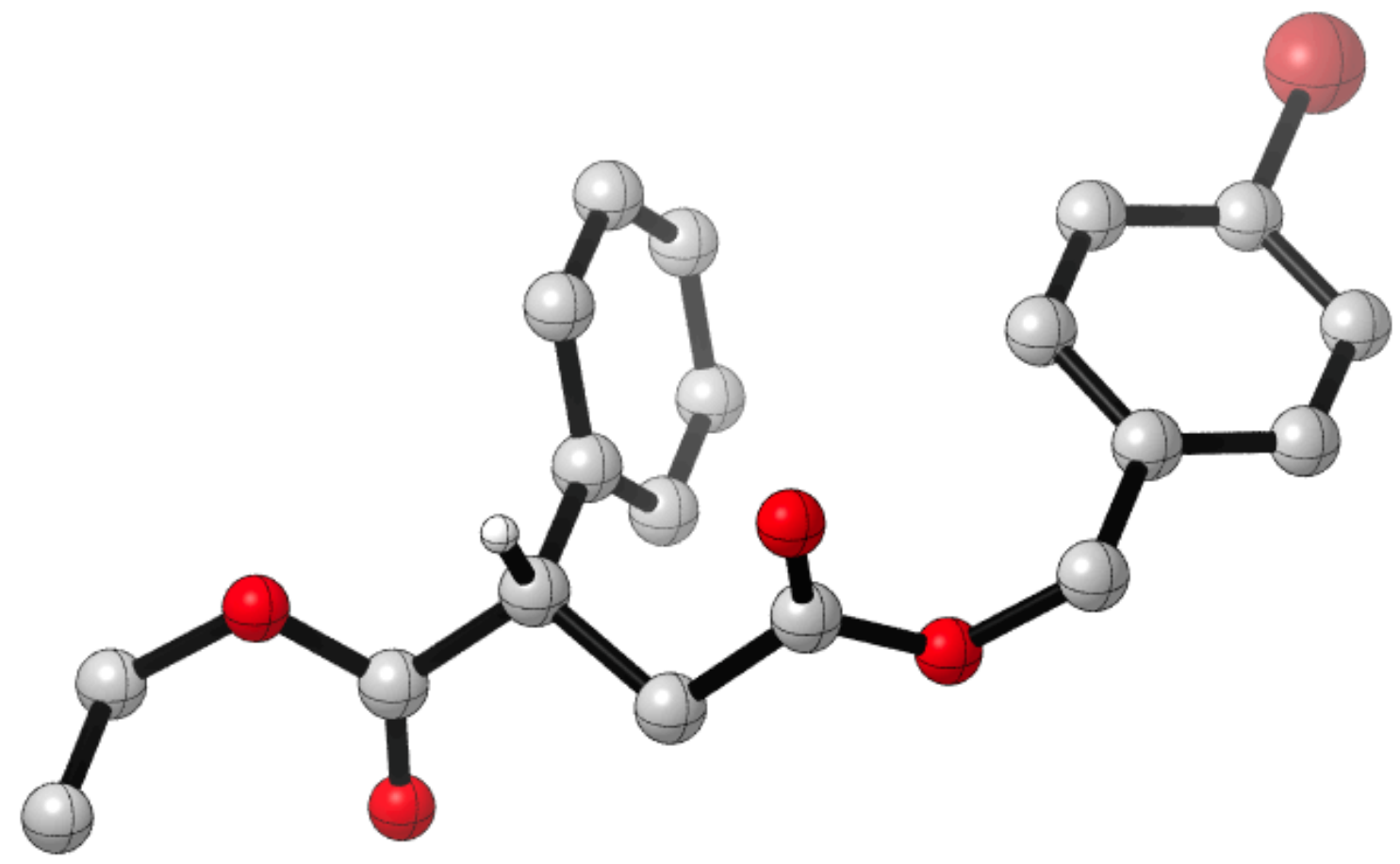

